\title{
The Effects of Extremely Low- Frequency Magnetic Fields on Mutation Induction in Mice
}

Thesis submitted for the degree of

Doctor of Philosophy

at the University of Leicester

by

James William Wilson

Department of Genetics

University of Leicester 
Doctor of Philosophy (2016)

University of Leicester

Department of Genetics

Title: The Effects of Extremely Low-Frequency Magnetic Fields on Mutation Induction in Mice

Author: James William Wilson (BSc, MSc)

Supervisor: Professor Yuri E. Dubrova

Number of Pages: 182

Number of Words: 43660 


\section{Abstract \\ The Effects of Extremely Low-Frequency Magnetic Fields on Mutation Induction in Mice}

James William Wilson

Extremely low-frequency magnetic fields (ELF-MF) are classified as possibly carcinogenic, despite inconsistent data and no plausible biological mechanism linking their universal exposure with childhood leukaemia and genotoxic effects.

Given discrepancies in mutagenic data and widespread public concern over genotoxic effects, this study was designed to provide an in-depth analysis of potential molecular changes induced by ELF-MF exposure in vivo. Seven-week old, BALB/C x CBA/Ca hybrid $F_{1}$ male mice were exposed to $50 \mathrm{~Hz}$ magnetic fields of 10,100 and $300 \mu \mathrm{T}$ for 2- or 15 hours. Blood and sperm DNA samples were collected 12 weeks post-exposure and mutation induction frequencies established at the Expanded Simple Tandem Repeat (ESTR) Ms6-hm loci using single-molecule PCR (SM-PCR). Likewise, Ms6-hm mutation induction frequencies were established in age-matched sham-treated hybrid males (control group) and those exposed to $1 \mathrm{~Gy}$ acute $\mathrm{X}$-rays (positive controls).

No significant increases in ESTR mutation frequencies were detected in either tissue at any ELF-MF exposure parameter compared to their sham-treated controls. Whilst a marginally significant increase was observed in the mutation induction frequency of pooled sperm data, these data should be regarded cautiously due to the lack of correlating dose-dependent responses. Conversely, germline and somatic ESTR mutation frequencies were significantly elevated in males exposed to acute $1 \mathrm{~Gy} \mathrm{X}$ rays.

These data were validated in a high-throughput microarray pilot study, whereby no significant alterations in gene expression in kidney cells of hybrid males were detected following ELF-MF exposure. In contrast, five transcripts were significantly up-regulated in the irradiated males.

Ultimately, these findings indicate that, within the analysed range of doses, the in vivo effects of ELF-MF exposure on mutation induction and gene expression are likely to be negligible. This study represents the first methodical attempt to determine mutation frequencies in vivo after continuous exposure to $50 \mathrm{~Hz}$ ELF-MFs up to $300 \mu \mathrm{T}$. 


\section{Acknowledgements}

Statistical analysis of microarray data was assisted with by Professor. Yuri Dubrova.

I would like to take this opportunity to express my utmost gratitude to my supervisor Professor Yuri Dubrova, for all his advice, guidance, and support throughout my time in the laboratory and the completion of this thesis.

It is also with heartfelt thanks that I would like to acknowledge my self-proclaimed adoptive work parents, Doctors Aneela Majid and Colin Glen.

Thanks to Aneela's encouragement and guidance, I gained experience with having my work reviewed and published, and was able to broadcast my research to the public through GENIE. Throughout the writing-up process, I have grown ever more grateful for this initial support.

Similarly, Dr. Colin Glen and Milly Veselis were invaluable in their advice and guidance. Their knowledge and experience of the assay employed within this project thoroughly advanced my understanding and hastened the progress I was able to make. However, and perhaps more importantly, I would like to thank them both for making the working atmosphere in the laboratory an enjoyable and entertaining one; workdays became somewhat duller and their absences towards the end of the project was sorely felt.

Finally, a special thanks to my friends and colleagues lan and Steven for all their moodlifting stories and support over lunches and coffee breaks, they enabled me to set about my afternoon's research with increased vigour.

Overall, these six people made my time in Leicester a most enjoyable experience and I am most thankful for their unwavering company and support.

I would further like to extend my thanks to the EMF Biological Research Fund for their financial support in presenting the Grant that funded this highly interesting project (BRT11/45). Additionally, I am grateful to Dr. Zenon Sienkiewicz and Jackie Haines for their contributions to the design of this project and also for their guidance, participation and support within every aspect of the work undertaken at Public Health England. Through their continuing support, they made my stay and work there a most valued experience.

I would also like to express my appreciation for my previous G7 lab colleagues: Dr. Ade Adeolou, Dr. Andre Gomez and Dr. Hamdy Abouzeid Ali for all their help, advice and co-operation in the initial phase of my project.

Enormous and heartfelt thanks must also go to both my parents who provided me with never-ending mental and physical support throughout the writing of this thesis - not to mention the endless flow of tea and coffee, without which my concentration and 
writing abilities would have diminished. I cannot imagine how infinitely more difficult completion of this project would have been without their support throughout this entire process.

Finally, to my friend and partner throughout it all, Catherine, my muse, my rock, you gave me the inspiration to keep ploughing forward, and unwavering support when the dark times set in. Your help will never be forgotten.

To all of you that assisted me throughout this process, I am eternally grateful.

It's finally done, thank you. 


\section{Table of Contents}

Table of Tables

Table of Figures ii

List of Abbreviations ............................................................................................... ii

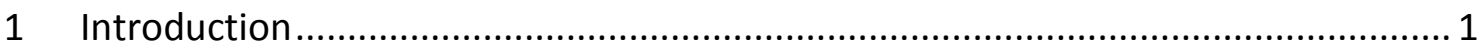

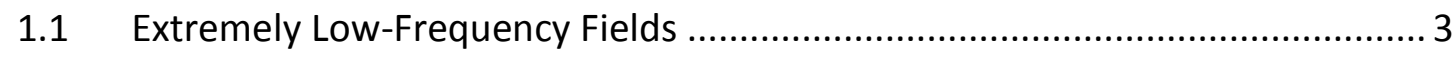

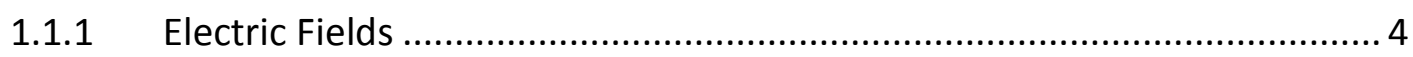

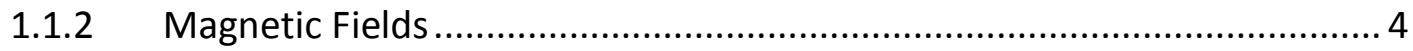

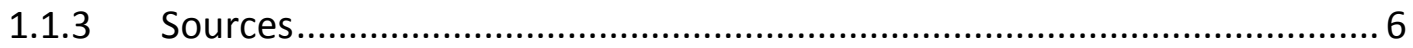

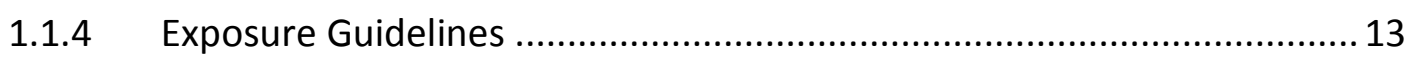

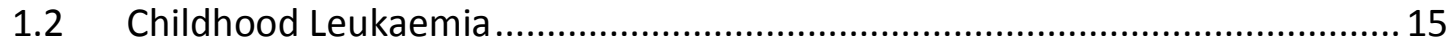

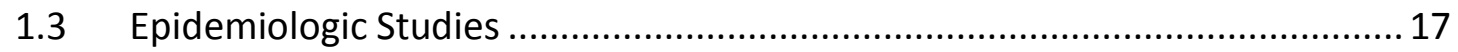

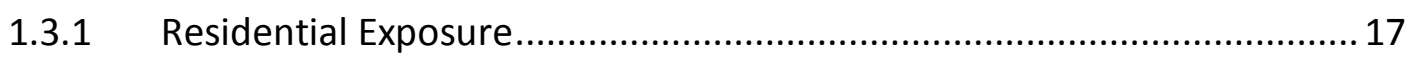

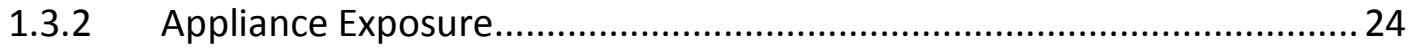

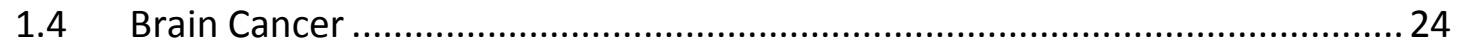

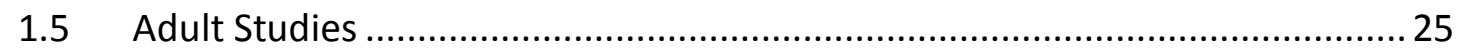

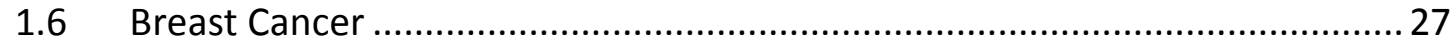

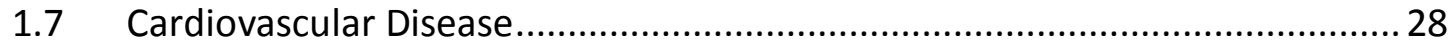

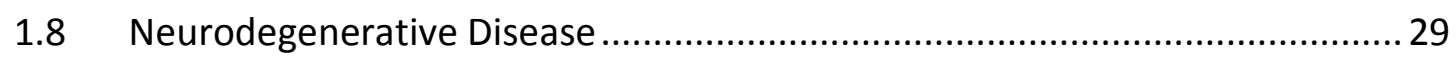

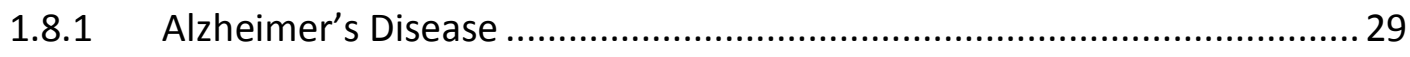

1.8.2 Amyotrophic Lateral Sclerosis ............................................................. 30

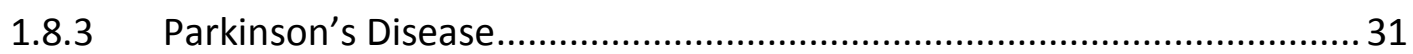

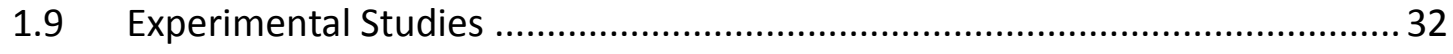

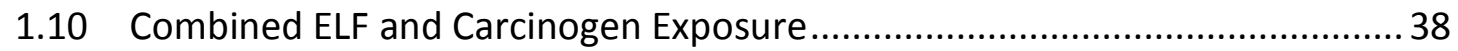

1.11 Experimental Evidence for Carcinogenicity of ELF-MF ................................ 41

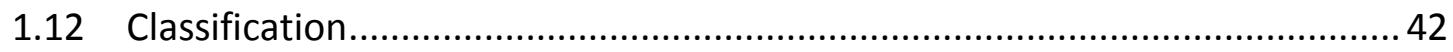

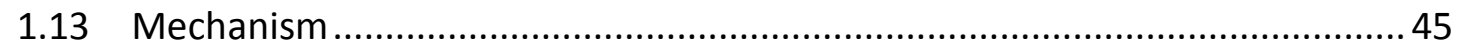

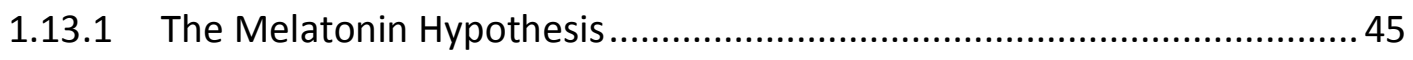

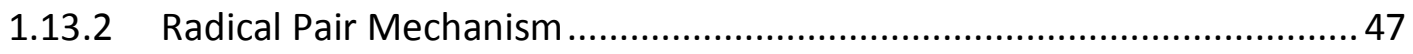

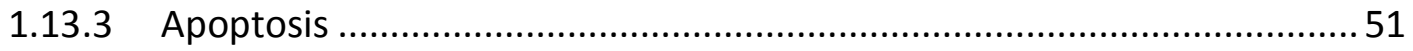

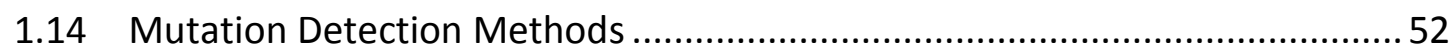

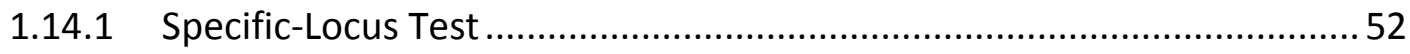




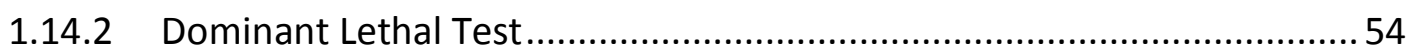

1.14.3 Hypervariable Tandem Repeat Regions................................................ 55

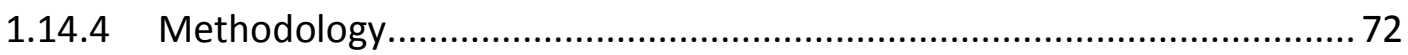

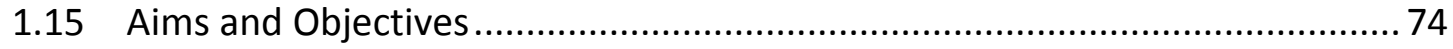

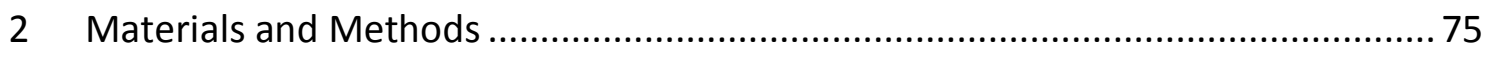

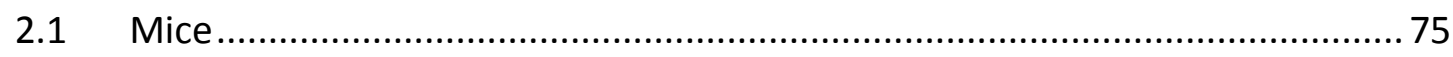

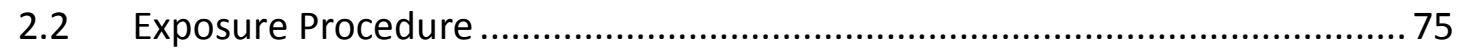

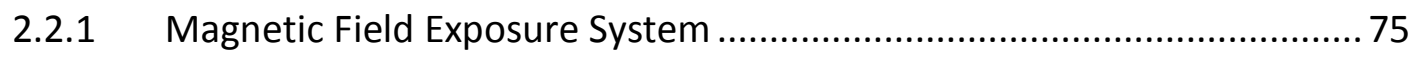

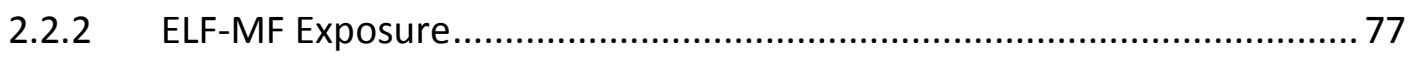

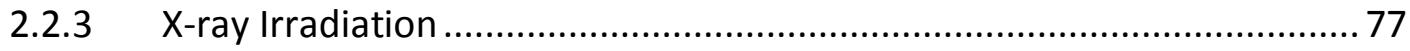

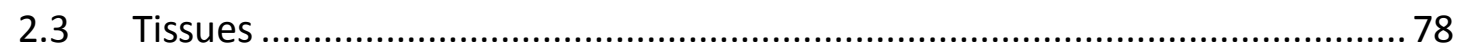

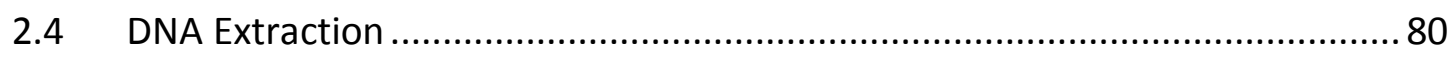

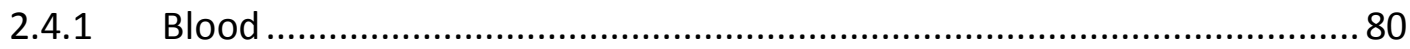

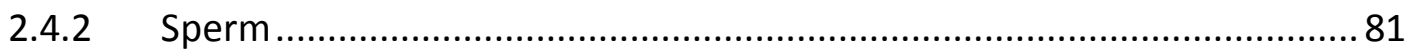

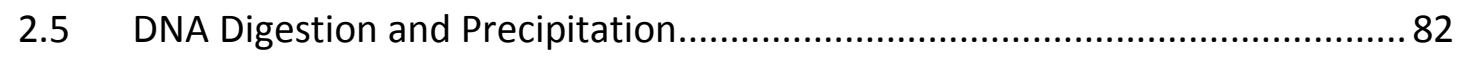

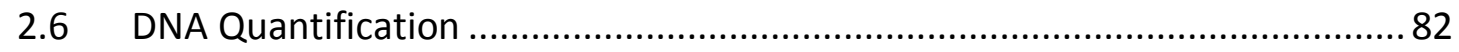

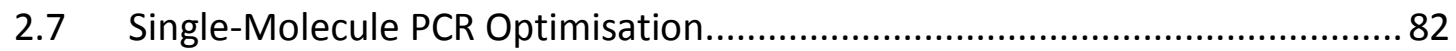

2.8 Poisson Analysis at the Single Molecule Level ............................................ 83

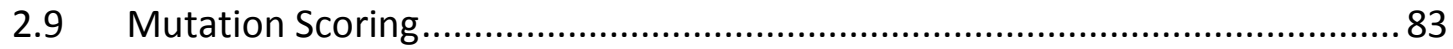

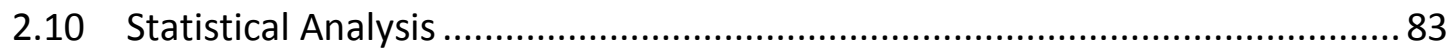

2.11 Ms6-hm Amplification and Autoradiograph Preparation ................................ 84

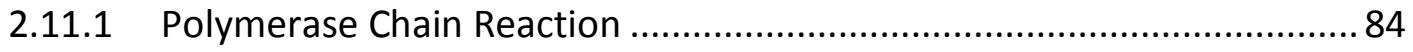

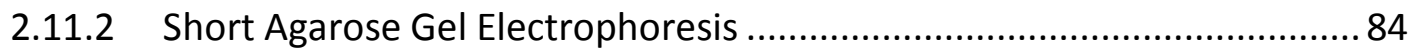

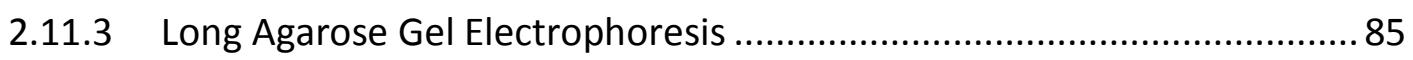

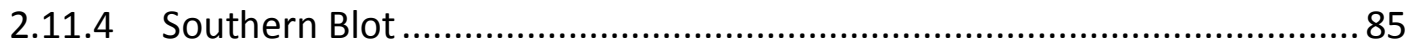

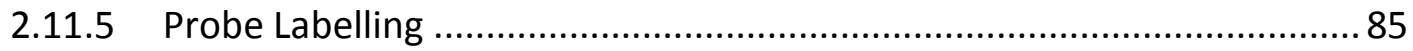

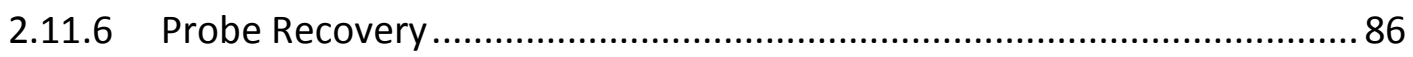

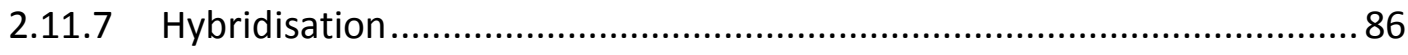

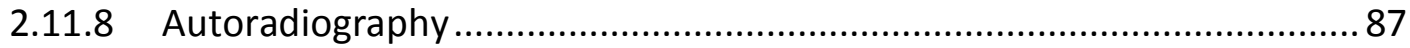

2.11.9 Mutation Scoring and Sizing of ESTR Mutants ................................... 87

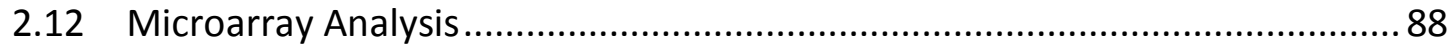

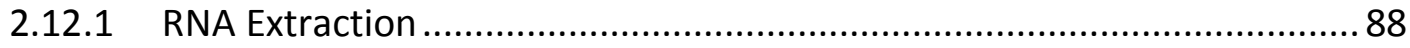




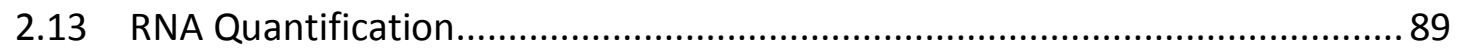

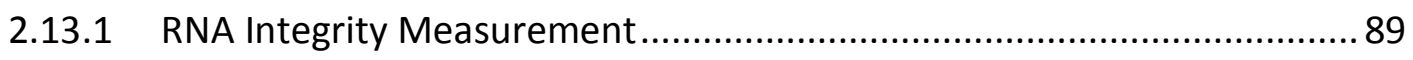

2.14 One-Colour Microarray-based Gene Expression Analysis .............................. 92

2.14.1 Template total RNA with Spike-In...................................................... 92

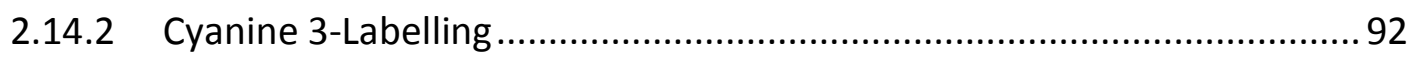

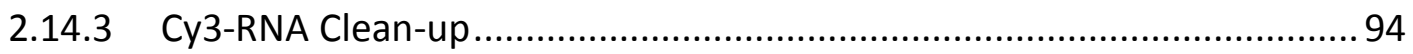

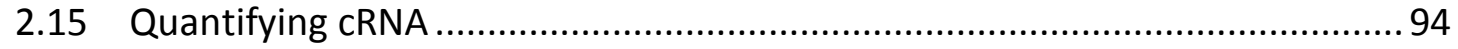

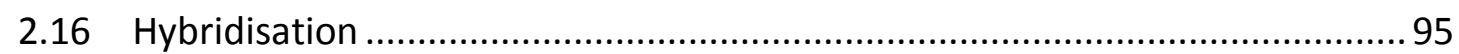

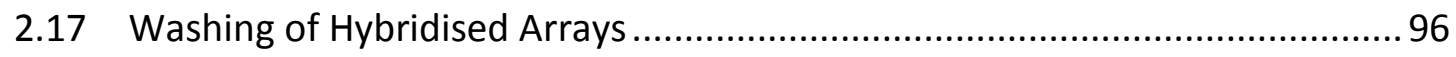

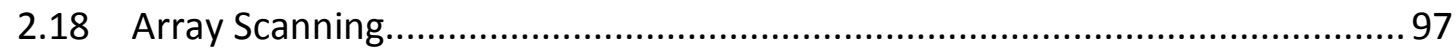

2.19 Data Normalisation \& Quality Control ............................................................. 97

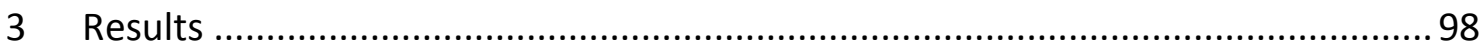

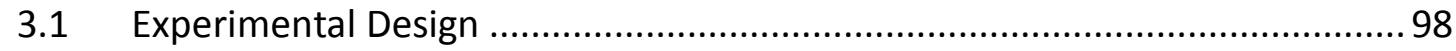

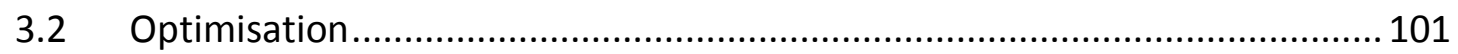

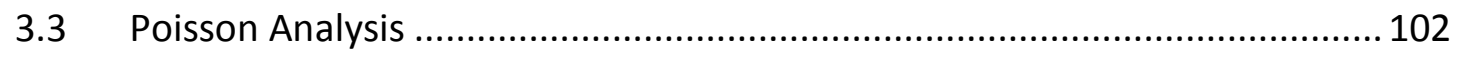

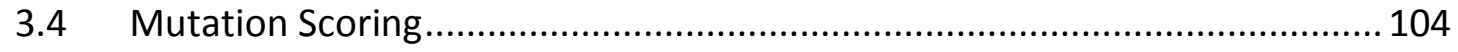

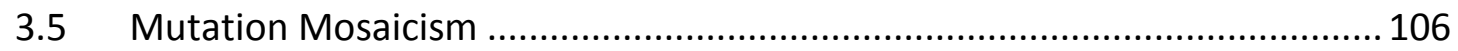

3.6 ESTR Mutation Frequencies in Sham-treated Males .................................. 107

3.7 ESTR Mutation Frequencies in Irradiated Males.......................................... 109

3.8 ESTR Mutation Frequencies in Males Exposed to $50 \mathrm{~Hz}$ Magnetic Fields ..... 109

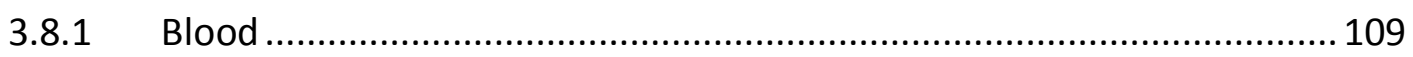

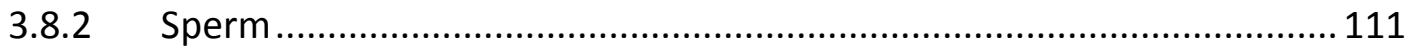

3.8.3 Combined ELF-MF effect............................................................. 113

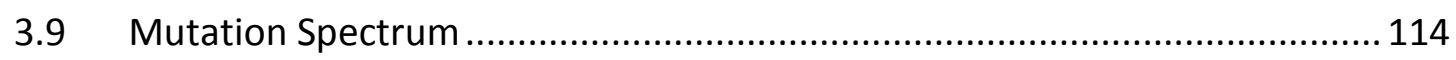

3.9.1 Size Spectra of Ms6-hm Mutations in Blood ....................................... 115

3.9.2 Size Spectra of Ms6-hm Mutations in Sperm ..................................... 117

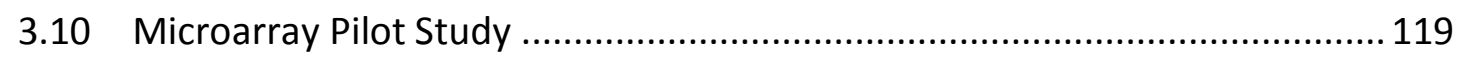

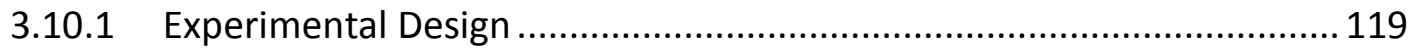

3.10.2 Gene Expression Analysis............................................................... 120

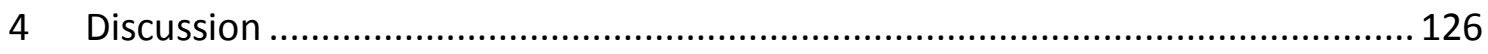

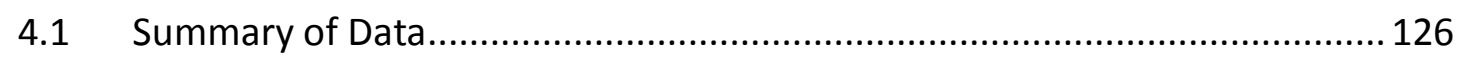

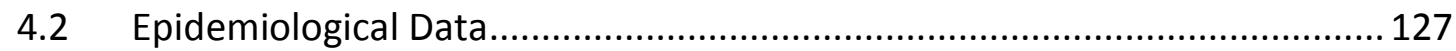




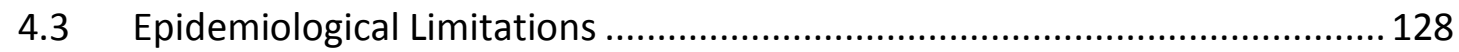

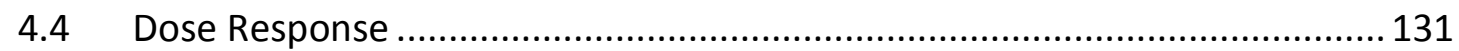

4.5 Comparative Analysis of Study Results .................................................... 133

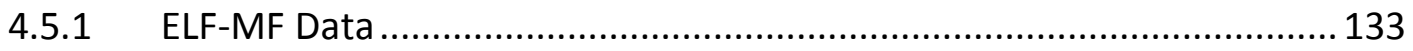

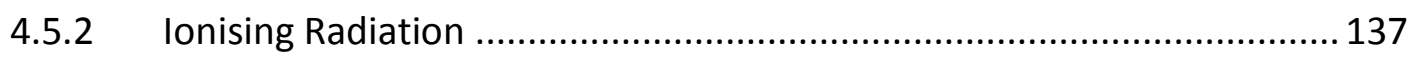

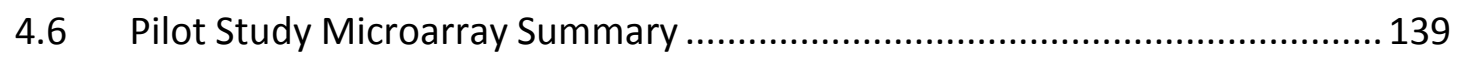

4.6.1 Comparative Analysis of Microarray Data ........................................... 139

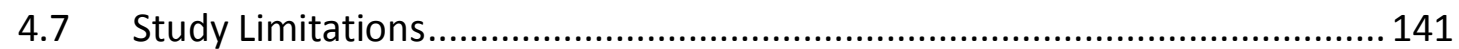

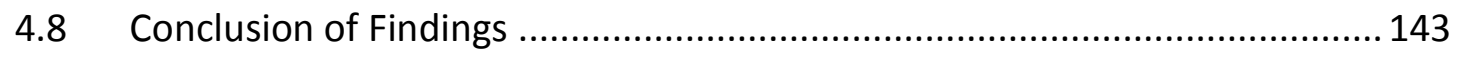

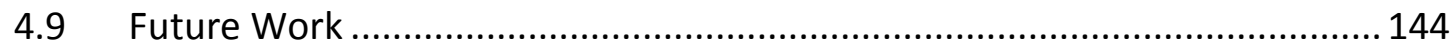

4.9.1 Microarray Analysis ......................................................................... 144

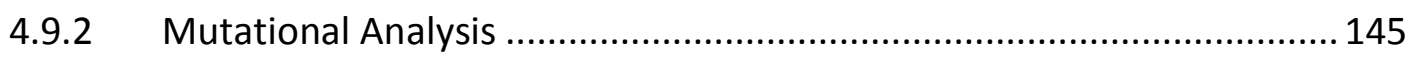

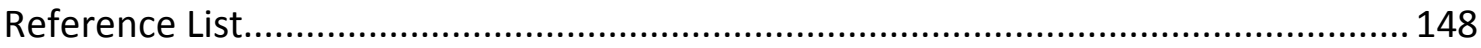




\section{Table of Tables}

Table 1. The magnetic fields from $50 \mathrm{~Hz}$ supplied household appliances....................... 10

Table 2. The magnetic fields from $60 \mathrm{~Hz}$ supplied household appliances..................... 11

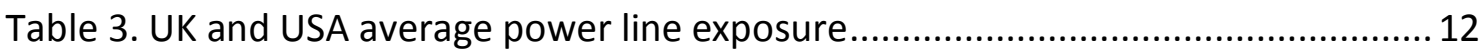

Table 4. Wire code assessment categories....................................................................... 19

Table 5. The ELF-MF time-weighted average exposures for electrical occupations ...... 27

Table 6. The IARC classification scale of carcinogenic risk to humans ........................... 44

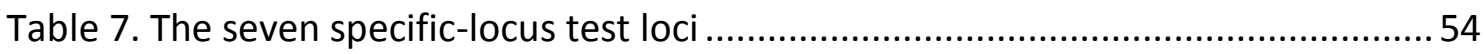

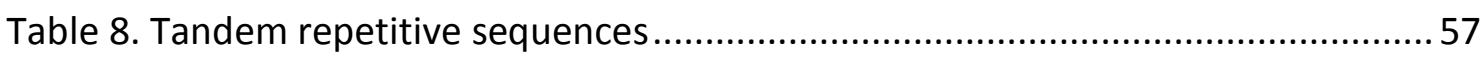

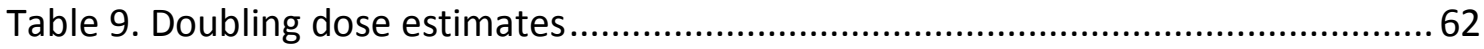

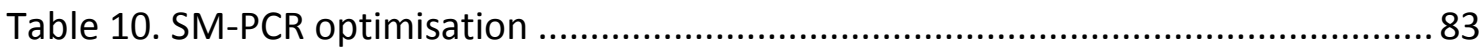

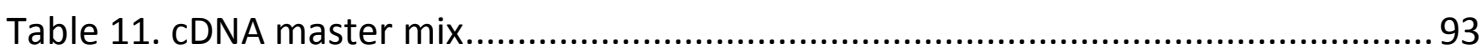

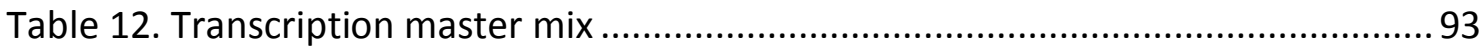

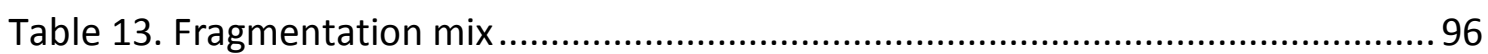

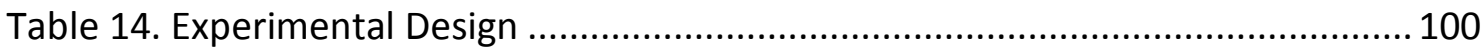

Table 15. ESTR mutation frequencies in sham-treated males.................................. 107

Table 16. Statistics for the difference between sham-treated groups ....................... 108

Table 17. ESTR mutation frequencies for all age-matched controls ............................ 109

Table 18. Summary of ESTR mutation data in blood ................................................ 110

Table 19. Summary of ESTR mutation data in sperm ................................................. 112

Table 20. ESTR mutation spectra of control and treated males in blood...................... 115

Table 21. ESTR mutation spectra of control and treated males in sperm ..................... 118

Table 22. Summary of up-regulated genes............................................................. 123 


\section{Table of Figures}

Figure 1. The Electromagnetic Spectrum.................................................................... 2

Figure 2. A simplified Two-Hit Model for potential CL onset ..................................... 16

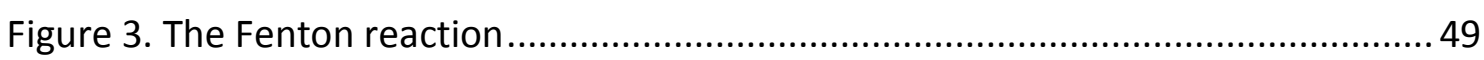

Figure 4. Diagrammatic representation of an insertion mutation caused by replication

slippage

Figure 5. Diagrammatic representation of a deletion mutation caused by replication

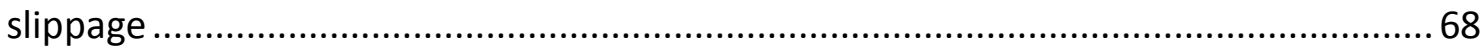

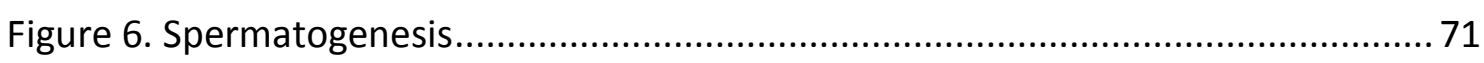

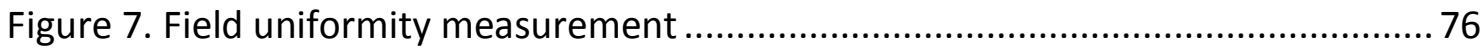

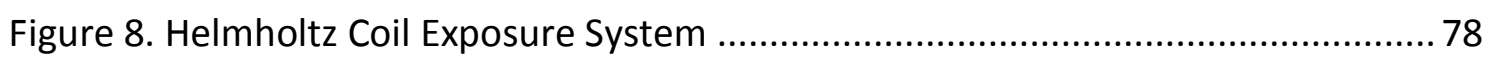

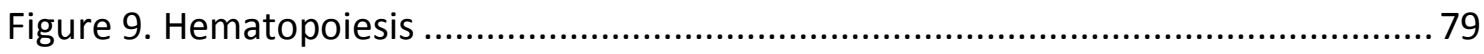

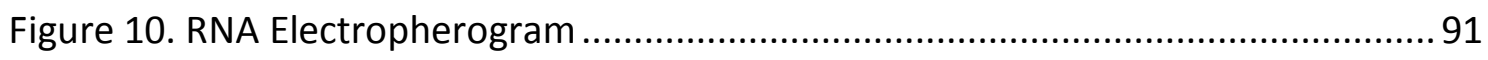

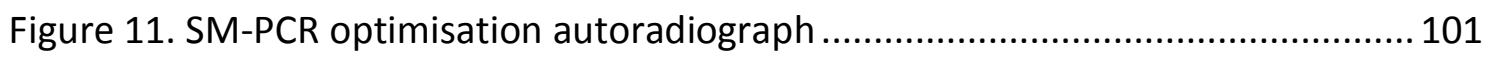

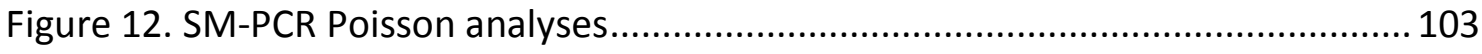

Figure 13. Mutation detection at the Ms6-hm ESTR locus....................................... 105

Figure 14. Mutational mosaicism at the Ms6-hm ESTR locus ................................... 107

Figure 15. ESTR mutation frequencies at the Ms6-hm locus in sham-treated

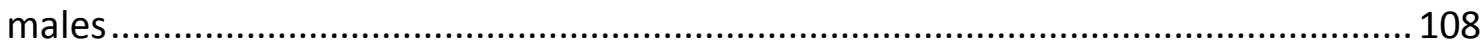

Figure 16. ESTR mutation frequencies in blood of exposed and matched

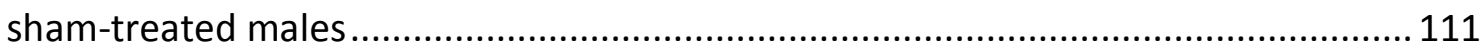

Figure 17. ESTR mutation frequencies in sperm of exposed and matched

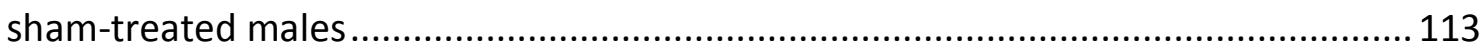

Figure 18. ESTR mutation frequencies in sham-treated and exposed males............... 114

Figure 19. Spectra of somatic ESTR mutations in sham-treated controls and exposed male mice

Figure 20. Spectra of germline ESTR mutations in sham-treated controls and exposed

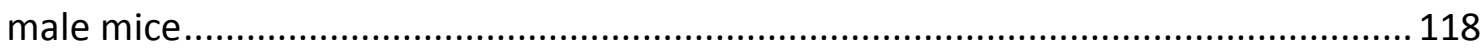

Figure 21. ELF-MF gene expression analysis..................................................... 121

Figure 22. Ionising radiation gene expression analysis .......................................... 122

Figure 23. Comparison of differentially expressed probes......................................... 124

Figure 24. Probability plot analysis comparing the exposure distributions between ELFMF and X-ray irradiated samples 126 


\section{List of Abbreviations}

\begin{tabular}{|c|c|}
\hline$\cdot \mathrm{OH}$ & Hydroxyl radical \\
\hline $\mathrm{OOH}$ & Superoxide radicals \\
\hline$\sim$ & Approximately \\
\hline $\boldsymbol{\mu}$ & Micro \\
\hline$\mu T$ & micro Tesla \\
\hline 6-TG ${ }^{r}$ & 6-thioguanine-resistant \\
\hline$A / m^{-1}$ & Amperes per metre \\
\hline$A C$ & Alternating current \\
\hline AD & Alzheimer's disease \\
\hline ALL & Acute lymphoblastic leukaemia \\
\hline ALS & Amyotrophic lateral sclerosis \\
\hline AML & Acute myeloid leukaemia \\
\hline B & Magnetic flux density \\
\hline BM & Bone marrow \\
\hline Bp & Base pair \\
\hline BP & Benzo(a)pyrene \\
\hline BSA & Bovine Serum Albumin \\
\hline CA & Chromosomal aberrations \\
\hline САT & Catalase \\
\hline CCTV & Closed circuit television \\
\hline cGY & centi-Gray \\
\hline $\mathrm{Cl}$ & Confidence interval \\
\hline CL & Childhood leukaemia \\
\hline $\mathrm{Cm}$ & Centimetre \\
\hline c-Myc & Cellular myelocytomatosis \\
\hline Cps & counts per second \\
\hline CRNA & Complementary RNA \\
\hline CTP & Cytidine triphosphate \\
\hline DB & Dilution buffer \\
\hline DC & Direct current \\
\hline dCTP & Deoxycytidine triphosphate \\
\hline DEPC & Diethylpyrocarbonate \\
\hline df & degrees of freedom \\
\hline DLT & Dominant lethal test \\
\hline DNA & deoxyribonucleic acid \\
\hline dNTP & deoxynucleoside triphosphate \\
\hline DSB & Double strand break \\
\hline EDTA & ethylene-diamine-tetra-acetic acid \\
\hline EF & Electric field \\
\hline EHF & Extremely high-frequency \\
\hline ELF & Extremely low-frequency \\
\hline ELF-MF & Extremely low-frequency magnetic field \\
\hline ENU & $\mathrm{N}$-ethyl-N-nitrosourea \\
\hline ES-1 cells & Human primary fibroblast cells from 6 year old male \\
\hline
\end{tabular}




\begin{tabular}{|c|c|}
\hline ESTR & Expanded simple tandem repeat \\
\hline eV & Electron-volts \\
\hline $\boldsymbol{F}$ & Frequency \\
\hline $\mathbf{F}_{1}$ & First filial generation \\
\hline $\mathbf{F}_{2}$ & Second filial generation \\
\hline FDR & False discovery rate \\
\hline G & Gauss \\
\hline G & Grams \\
\hline GIT & Gastrointestinal tract \\
\hline GM & Geometric mean \\
\hline GSH & Glutathione \\
\hline Gy & Gray \\
\hline Gy $\min ^{-1}$ & Grays per minute \\
\hline H & Hours \\
\hline $\mathbf{H}$ & Magnetic field strength \\
\hline $\mathrm{H} / \mathrm{m}^{-1}$ & Henry per metre \\
\hline $\mathrm{H}_{2} \mathrm{O}$ & Water \\
\hline $\mathrm{H}_{2} \mathrm{O}_{2}$ & Hydrogen peroxide \\
\hline $\mathrm{HCC}$ & High-current configuration \\
\hline $\mathrm{HCl}$ & Hydrochloric acid \\
\hline HF & High frequency \\
\hline HL-60 cells & Human promyelocytic leukaemia cells \\
\hline$H m-2$ & Human microsatellite-2 \\
\hline HPRT & hypoxanthine-guanine phosphoribosyl transferase \\
\hline HR-1d cells & Human primary fibroblast cells from 42 year old male \\
\hline HRV & Heart rate variability \\
\hline HSC & Hematopoietic stem cell \\
\hline HSP & Heat shock protein \\
\hline $\mathrm{Hz}$ & Hertz \\
\hline IARC & International Agency for Research on Cancer \\
\hline ICNIRP & International Commission on Non-Ionising Radiation Protection \\
\hline iPMS & isopropyl methanesulfonate \\
\hline $\mathrm{J} / \mathrm{cm}^{2}$ & Joules per centimetre squared \\
\hline JEM & Job exposure matrix \\
\hline Kb & Kilobases \\
\hline Km & Kilometres \\
\hline kV & Kilovolts \\
\hline $\mathbf{L}$ & Litre \\
\hline LCC & Low-current configuration \\
\hline LF & Low frequency \\
\hline lincRNAs & Long intergenic non-coding ribonucleic acids \\
\hline LTR & Long terminal repeat \\
\hline M & Molar \\
\hline m- & Milli \\
\hline M5s cells & Mouse embryonic skin cells \\
\hline MaLR & Mammalian apparent LTR-retrotransposon \\
\hline MCF10A cells & Human breast epithelial cells \\
\hline
\end{tabular}




\begin{tabular}{|c|c|}
\hline MeWo cells & Cultured human melanoma cells \\
\hline MF & Medium Frequency (Electromagnetic Spectrum (P2)) \\
\hline MF & Magnetic field \\
\hline Mm & milli metre \\
\hline MN & Micronuclei \\
\hline MNNG & $\mathrm{N}$-methyl-NO-nitro-N-nitrosoguanidine \\
\hline MRC-5 cells & Human primary foetal lung fibroblast cells \\
\hline Ms6-hm & Minisatellite-6-hypermutable \\
\hline MT & Mouse transposon \\
\hline $\mathbf{N}$ & Nano \\
\hline $\mathrm{NaAc}$ & Sodium acetate \\
\hline $\mathrm{NaCl}$ & Sodium Chloride \\
\hline $\mathrm{NaOH}$ & Sodium Hydroxide \\
\hline NEB & New England Biolabs \\
\hline No & Number \\
\hline nT & nano Tesla \\
\hline Nt & Nucleotide \\
\hline${ }^{\circ} \mathrm{C}$ & degrees Celsius \\
\hline OHCC & Ordinary high-current configuration \\
\hline OLCC & Ordinary low-current configuration \\
\hline OR & Odds ratio \\
\hline ORR-1 & Origin-region repeat 1 \\
\hline $\mathbf{P}$ & Pico \\
\hline $\boldsymbol{P}$ & Probability \\
\hline PAR & Population attributable risk \\
\hline PBS & Phosphate-buffered saline \\
\hline PCR & Polymerase chain reaction \\
\hline PD & Parkinson's disease \\
\hline pmol & Pico-mole \\
\hline PMR & Proportionate mortality ratios \\
\hline RAT-1 cells & Rat fibroblast cells \\
\hline Rcf & Relative centrifugal force \\
\hline RF & Radiofrequency \\
\hline RIN & RNA integrity number \\
\hline RNA & Ribonucleic acid \\
\hline RNA-Seq & RNA sequencing \\
\hline ROS & Reactive oxygen species \\
\hline RPM & Radical pair mechanism \\
\hline Rpm & Revolutions per minute \\
\hline $\mathbf{R R}$ & Relative risk \\
\hline RT & Room temperature \\
\hline RT-PCR & Reverse transcription-polymerase chain reaction \\
\hline s.e. & Standard error \\
\hline SCE & Sister chromatid exchange \\
\hline SDS & Sodium dodecyl Sulphate \\
\hline SHF & Super high-frequency \\
\hline SI & Système International \\
\hline
\end{tabular}




\begin{tabular}{|c|c|}
\hline SLT & Specific-locus test / Russell 7-locus test \\
\hline SM & Single-molecule \\
\hline SM-PCR & Single-molecule polymerase-chain reaction \\
\hline SOD & Superoxide dismutase \\
\hline SP-PCR & Small-pool polymerase chain reaction \\
\hline SSB & Single-strand breaks \\
\hline SSC & Saline-Sodium Citrate \\
\hline$T$ & Student $t$ test value \\
\hline $\mathbf{T}$ & Tesla \\
\hline TAE & Tris-acetate-EDTA \\
\hline TBE & Tris-Borate-EDTA \\
\hline$T$-stock mouse & Genetic tester stock mouse \\
\hline $\mathbf{U}$ & Unit \\
\hline UG & Underground cables \\
\hline UHF & Ultra-high frequency \\
\hline UK & United Kingdom \\
\hline UKCCS & UK Childhood Cancer Study \\
\hline USA & United States of America \\
\hline UV & Ultraviolet \\
\hline UVW & Human glioma cells \\
\hline $\mathbf{V}$ & Volts \\
\hline $\mathrm{V} / \mathrm{m}^{-1}$ & Volts per metre \\
\hline VF & Voice frequency \\
\hline VH25 cells & Human skin fibroblast cells \\
\hline VHCC & Very high-current configuration \\
\hline VHF & Very high-frequency \\
\hline VLCC & Very low-current configuration \\
\hline VLF & Very low-frequency \\
\hline $\begin{array}{l}\text { WI-38 cells } \\
\alpha-{ }^{32} \mathrm{P}-\mathrm{dCTP}\end{array}$ & $\begin{array}{l}\text { Embryonic human lung fibroblast cells } \\
\text { deoxycytidine triphosphate-alpha-32-phosphate }\end{array}$ \\
\hline VH2AX & Phosphorylated form of histone $\mathrm{H} 2 \mathrm{AX}$ \\
\hline$\delta$ & $4 \pi \times 10^{-7}$ \\
\hline$\lambda$ & Wavelength \\
\hline$x^{2}$ & Chi-squared \\
\hline
\end{tabular}




\section{Introduction}

Human exposure to the numerous forms of electromagnetic radiation is not a new phenomenon, in fact naturally-occurring fields of different strengths are omnipresent within the environment (IARC Working Group on the Evaluation of Carcinogenic Risks to Humans, 2000; IARC Working Group on the Evaluation of Carcinogenic Risks to Humans, 2002; IARC Working Group on the Evaluation of Carcinogenic Risks to Humans, 2013). Encompassed within the electromagnetic spectrum (Figure 1), the various forms of electromagnetic fields are separated according to the quantity of energy they emit and the way in which they interact with physical matter. Energy corresponding to the fields' properties is emitted from the source and propagated through space or an alternative medium by vectors, in the form of time-varying electric (E) and magnetic (M) fields that act as wave-like modes of transport (IARC Working Group on the Evaluation of Carcinogenic Risks to Humans, 2013). Owing to the different physical properties of the various electromagnetic fields, the electromagnetic spectrum is sub-divided into two further categories: ionising and non-ionising radiation. lonising radiation such as $\mathrm{X}$-rays and $\mathrm{Y}$-rays are often referred to in terms of photon energy (eV). Both possess sufficient energy capable of causing either direct or indirect damage to DNA, lipids and protein through both the removal and ionisation of electrons from their orbit and/or the formation of free radicals (Powell \& McMillan, 1990; IARC Working Group on the Evaluation of Carcinogenic Risks to Humans, 2000). Alternatively, fields characterised as static, extremely low-frequency (ELF), radiofrequency ( $\mathrm{RF}$ ), microwaves, infra-red, visible light and ultraviolet fields are known as non-ionising radiation and do not possess the same quantity of energy within a single quantum needed to ionise an atom or molecule. Furthermore, the field strength related to the electric and magnetic components is described by either the frequency $(f)$ as is the case with static and extremely low-frequency fields, or the wavelength $(\lambda)$. The frequency of a field is measured in hertz $(\mathrm{Hz})$ and depicts the number of sinusoidal cycles the wave completes per second (IARC Working Group on the Evaluation of Carcinogenic Risks to Humans, 2013). Alternatively, the field strength for radiofrequency to infra-red fields is characterised by the wavelength $(\lambda)$, which relates to the distance in metres between successive peaks of the wave (IARC Working 
Group on the Evaluation of Carcinogenic Risks to Humans, 2013). It should be noted that frequency is inversely proportional to wavelength as illustrated by Equation 1, where $c$ represents the speed of light in a vacuum $\left(3 \times 10^{8} \mathrm{~m} / \mathrm{s}\right)$ (IARC Working Group on the Evaluation of Carcinogenic Risks to Humans, 2002).

\section{Equation 1}

$$
\lambda=\frac{c}{f}
$$

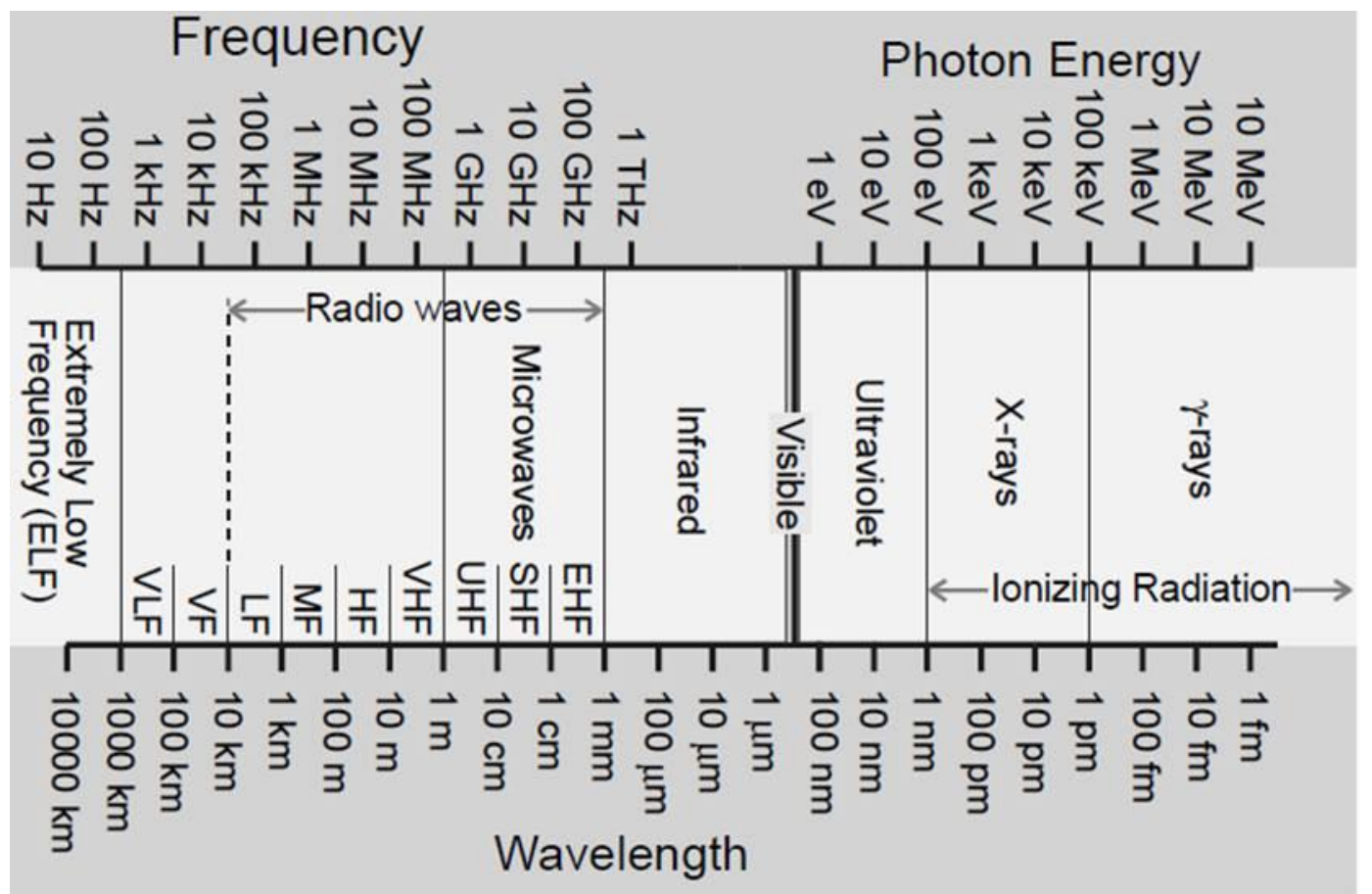

Figure 1. The Electromagnetic Spectrum. The frequency (expressed in hertz $(\mathrm{Hz})$ ) increases from left to right and the category to which they correspond is indicated. Electrical appliances operate in the extremely low-frequency range $50-60 \mathrm{~Hz}$, depending on the country of residence. The abbreviations VLF, VF, LF, MF, HF, VHF, UHF, SHF and EHF represent very low-frequency, voice frequency, low frequency, medium frequency, high frequency, very high-frequency, ultra-high frequency, super high-frequency and extremely high-frequency. Thereafter, from radiowaves until ultraviolet, categories are referred to by wavelength and expressed in metres. Ionising radiation is referred to in terms of photon energy expressed in electron-volts (eV) (IARC Working Group on the Evaluation of Carcinogenic Risks to Humans, 2000). Figure taken from IARC Working Group on the Evaluation of Carcinogenic Risks to Humans, 2013.

Exposure of humans to electromagnetic fields has grown considerably. Indeed, since the $19^{\text {th }}$ century public exposure to man-made electromagnetic fields has been steadily increasing in conjunction with advancements in medical and industrial equipment, 
development of telecommunication systems and the growth of electrical power generation and transmission. Thus, the growth of our technology orientated society and increased reliance upon electrical power has not only led to changes in social behaviour, but also constant environmental exposure to a complex mix of electromagnetic fields. Of particular interest to the media (Edwards, 1990), public and scientific communities (Feychting et al., 2005), is exposure to the extremely lowfrequency (ELF) region of the electromagnetic spectrum (Figure 1). Primarily classified as corresponding to frequencies from $30-300 \mathrm{~Hz}$, the scientific literature is often expanded to include the frequency range: $0-100 \mathrm{kHz}$ (IARC Working Group on the Evaluation of Carcinogenic Risks to Humans, 2002). However, frequencies corresponding to ELF are predominantly associated with the generation, transmission and use of electrical energy, whereby weak electric (EF) and magnetic fields (MF) are produced, which oscillate at a frequency of $50 \mathrm{~Hz}$ in Europe and Asia and $60 \mathrm{~Hz}$ in the USA (Feychting \& Ahlbom, 1995). Concern has increased amongst reports of a potential association between extended ELF exposure and a wide range of adverse health effects relating to: adult and childhood cancers (Wertheimer \& Leeper, 1979), depression and suicide (Ahlbom, 2001), neurodegenerative diseases (Hakansson et al., 2003) and compromised reproductive capacity (Huuskonen et al., 1998; Ahlbom et al., 2001; Panagopoulos et al., 2013). Indeed, potential adverse health effects were first illustrated in a series of studies conducted by the former Soviet Union in the early 1960's. Russian substation workers complained of increased headaches and cardiovascular disorders including abnormal heart rates and arrhythmia (reviewed in (Bonnell, 1982; Fatigoni et al., 2005)). However, any potential follow-up studies were postponed under the premise that the problem was too complex to analyse (reviewed by Santini et al., (2009)). It was not until the publication of Wertheimer and Leeper's (1979) ground-breaking study linking continuous ELF exposure to an increased incidence of childhood leukaemia that extensive investigations started within this area of research, principally in the form of epidemiologic studies.

\subsection{Extremely Low-Frequency Fields}

The fundamentals of electromagnetism as described by James Clerk Maxwell's equations state that the presence of a time-varying electric field produces a time- 
varying magnetic field and vice versa, which then couple together to form a propagating electromagnetic wave. However, the formation of an electromagnetic wave typically only occurs at high frequencies. In contrast, the production of weak electric and magnetic fields which oscillate within the frequency range of ELF, consist of very long wavelengths ( 6000 kilometres $(\mathrm{km})$ at $50 \mathrm{~Hz}$ and $5000 \mathrm{~km}$ at $60 \mathrm{~Hz}$ ), thus the interdependence of the electric and magnetic field components becomes weaker (IARC Working Group on the Evaluation of Carcinogenic Risks to Humans, 2013). As a result the EF and MF propagate, in effect, uncoupled as if arising from independent sources (Fatigoni et al., 2005).

\subsubsection{Electric Fields}

Electric fields arise from the presence of electric charges, regardless of the flow of an electrical current (IARC Working Group on the Evaluation of Carcinogenic Risks to Humans, 2002). Subsequently, electric fields are related to voltages rather than currents and measured as electric field strength, which is expressed in the Système International (SI) unit volts per metre $\left(\mathrm{V} / \mathrm{m}^{-1}\right)$ (International Commission on NonIonizing Radiation Protection, 1998). Furthermore, electric fields are easily perturbed by materials hence buildings are able to reduce the electric field within them from an external source. Overall, this not only makes obtaining a reliable measure of personal exposure difficult but researchers have also noted that any measurement of the extremely low-frequency electric field component is essentially non-existent (BurdakRothkamm et al., 2009). Consequently, any epidemiologic studies which attempt to analyse the effects of external power lines are inherently studies exclusively pertaining to magnetic fields (Savitz, 1995). Similarly, Focke et al. (2010) has provided direct experimental evidence ruling out ELF electric fields as a possible carcinogen or contributing factor. The emphasis of experimental investigations has therefore been placed on determining the potential adverse health effects linked to exposure to the ELF-MF component exclusively (Tenforde, 1992).

\subsubsection{Magnetic Fields}

In contrast, magnetic fields arise from the motion of electric charges or more simply, the flow of electrical current (International Commission on Non-lonizing Radiation Protection, 1998). For that reason, magnetic fields are representative of the electric 
current in a system, irrespective of the voltage. Since electrical current is supplied as alternating current $(A C)$, in the form of a sinusoidal wave, the magnitude of the electric current determines the magnetic field (Kheifets \& Oksuzyan, 2008). Within the scientific literature the magnitude of a magnetic field is predominantly described as its magnetic flux density (B), expressed in the SI units of tesla (T) (IARC Working Group on the Evaluation of Carcinogenic Risks to Humans, 2002). However it is not uncommon, especially within the earlier studies published in the USA, to see the magnetic flux density displayed as gauss (G). Also since magnetic fields are generated by the movement of charged particles, they are occasionally, although less commonly, displayed as magnetic field strength $(\mathbf{H})$ and expressed as amperes per metre $\left(\mathrm{A} / \mathrm{m}^{-1}\right)$ (IARC Working Group on the Evaluation of Carcinogenic Risks to Humans, 2002). Despite this variation in SI units, these measurements are easily interchangeable with $1 \mathrm{G}$ being equivalent to $10^{-4} \mathrm{~T}$, while magnetic flux density and magnetic field strength are related by Equation 2 (International Commission on Non-lonizing Radiation Protection, 1998).

\section{Equation 2}

$$
\boldsymbol{B}=\boldsymbol{\delta} \boldsymbol{H}
$$

Where $\delta$ represents the constant of proportionality (the magnetic permeability) in a vacuum and air $\left(4 \pi \times 10^{-7}\right)$ and is expressed in Henry per metre $\left(\mathrm{H} / \mathrm{m}^{-1}\right)$ (International Commission on Non-Ionizing Radiation Protection, 1998). As a conversion $1 \mathrm{~A} / \mathrm{m}^{-1}$ equates to $1.25 \mu \mathrm{T}$. Furthermore, in contrast to electric fields, magnetic fields are not easily shielded and thus can easily penetrate the body: only ferromagnetic materials such as iron, which are highly susceptibility to magnetisation, can obstruct them (Fatigoni et al., 2005; Miller \& Green, 2010). This represents an issue in today's world in that, not only do ELF fields occur naturally within the environment, but since the first power station arose in 1882 (Kheifets \& Oksuzyan, 2008), global industrialisation and the extended use of electricity have resulted in their increase. Consequently, the presence of extremely low-frequency fields is universal, including within households, in schools and at work. As such, extensive research has been conducted to clarify the 
plethora of links between ELF-MF exposure and adverse biological effects, particularly carcinogenesis.

\subsubsection{Sources}

\subsubsection{Naturally Occurring Fields}

Although representative of a static field, the most recognisable naturally occurring field is the Earth's magnetic field. Estimated to be around $50 \mu \mathrm{T}$, the geomagnetic field is not constant, but instead is subject to continuous fluctuations. Location is instrumental in the determination of total field intensity, at a maximum of $67 \mu \mathrm{T}$ at the magnetic poles the intensity diminishes towards the equator $(30 \mu \mathrm{T})$. Additionally, the geomagnetic field is known to fluctuate as a result of lunar and seasonal variations (Barnes \& Greenebaum, 2007). Similarly, time-varying magnetic fields in the ELF range are also present throughout the atmosphere as a product of solar and lunar effects on ion currents in the upper atmosphere (Tenforde, 1992). There is, however, also a continuous global presence of extremely low-frequency electromagnetic waves within the geomagnetic field as a result of a number of very low-intensity signals called Schumann resonances. These fields are generated by thunderstorms and lighting discharges within the resonant cavity formed between the Earth and the ionosphere. Lightning discharges create electromagnetic standing waves that cover the ELF spectrum with broad peaks of fading amplitude predominantly at a frequency of 7.83 $\mathrm{Hz}$, but also resonance frequencies of $14.1,20.3,26.4$, and 32.5 ranging up to $250 \mathrm{~Hz}$ (Chand et al., 2009). These waves are then transmitted around the globe through reflection between the lower boundary of the ionosphere and the earth's surface, the same mechanism through which the basis of distance radio communications are formed (Cherry, 2002). As the Schumann resonance forms electromagnetic waves, it can be detected as electric or magnetic micro-pulses. The electric component of which consists of $\sim 0.01 \mathrm{~V} / \mathrm{m}^{-1}$, while the magnetic fields amount to between 1 and $10 \mathrm{nT}$ (Funk et al., 2009).

Artificial ELF-MFs however represent strengths many thousands of magnitude greater than those arising naturally (IARC Working Group on the Evaluation of Carcinogenic Risks to Humans, 2002). Thus, the main sources of human ELF-MF exposure occur both through background fields originating from distant high-voltage overhead and 
underground power distribution lines and power transmission lines, and also fields resulting from the use of electrical appliances and devices, regardless of whether it be in an occupational (high levels of exposure over a short duration) or residential environment (generally lower exposure over an extended period of time).

\subsubsection{Artificial Fields}

\subsection{Background Fields}

Background magnetic fields within residences in many countries generally arise from the distribution lines supplying them electricity. Additionally, background fields can also arise within homes residing in close proximity to high-voltage transmission lines. The magnitudes of these fields will also be larger than those produced from the lower voltage distribution lines, although very few residences are located near such highvoltage transmission lines (Chapter 1.1.3.2.3) (Swanson \& Kaune, 1999).

Measurements of background fields have been conducted in many countries including Australia (Karipidis, 2015), North America (Linet et al., 1997) and the UK (Coghill et al., 1996), but studies in the rest of Europe often present poor degrees of geographical coverage (Grellier et al., 2014). The background fields recorded in Australia were in general, comparable with the USA (50-60 nT) (Karipidis, 2015). There are, however, differences in background fields between the USA and the UK with background fields within the UK being approximately 1.5 to 1.9 times weaker than those that resonate in North America (Swanson \& Kaune, 1999). The daily average background field in the USA was measured over a 24-hour period in eight individual studies as ranging from 47-99 nT (reviewed in Swanson \& Kaune, (1999)). However, uncertainties relating to the measurements made within these studies prevented the formation of a single, unambiguous approximation, leaving the authors to estimate that backgrounds fields within the USA typically present a geometric mean (GM) within the range of 60-70 nT (Swanson \& Kaune, 1999). However, in a study conducted by Zaffanella, (1992) which is considered the most representative study of background fields within the USA, spot measurements performed in the centre of most rooms in each home yielded a GM field of $56 \mathrm{nT}$ with only $10 \%$ of residences registering background levels above $0.2 \mu \mathrm{T}$ (Kheifets et al., 2005). Conversely, studies conducted in the UK (Merchant et al., 1994; 
Coghill et al., 1996; Preece et al., 1996) provided a GM of $38 \mathrm{nT}$, with the accurate value believed to be between 36-39 nT (Swanson \& Kaune, 1999).

The variation between these countries is likely a consequence of different electrical supply voltages. Since magnetic fields arise from the flow of electrical currents, consumers in the UK use approximately double the final distribution voltage than in the USA (220-240 volts (V) versus 110 volts, respectively), meaning that the currents in the UK are halved for the same power (Merchant et al., 1994). The background fields which are generated are, in general, uniformly present throughout the home. This was demonstrated in a study by Preece and co-authors, (1997) who failed to illustrate any variation between rooms when the background fields were measured in the centre of every room of 50 residences. In this study measurements were taken with both the power on and off, recording values of $0.017 \pm 0.003 \mu \mathrm{T}$ and $0.012 \pm 0.002 \mu \mathrm{T}$ respectively (Preece et al., 1997). Yet, since magnetic fields arise from electrical currents they vary proportionally and according to electrical demand. This has led to variations in readings between different times of day (Banks et al., 2002; Karipidis, 2015), and some studies have also illustrated seasonal differences whereby summer fields are in excess of those measured in winter (Banks et al., 2002). Uncertainties remain as to whether this is in fact founded, since other studies failed to illustrate any such differences (Kaune \& Zaffanella, 1994; Karipidis, 2015).

Alongside ELF-MF exposure from ubiquitous low-intensity background fields, approximately $30-50 \%$ of total ELF-MF exposure resides in fields generated through the use of electrical domestic appliances and devices (Kheifets et al., 2005; Repacholi, 2012). Unlike the background fields, ELF-magnetic fields produced by appliances are constantly more intense, yet only experienced intermittently.

\subsection{Electrical Appliances}

During use, each appliance produces an elevated field within its immediate vicinity. As such humans are exposed to the highest magnetic flux densities when in close proximity to domestic appliances, particularly those containing looped heating elements, motors or transformers (Merchant et al., 1994). Magnetic fields produced by appliances are typically localised and decrease as the inverse cube of distance from the source, thus at distances of 1-2 metres they are similar to background fields (Lacy- 
Hulbert et al., 1998). Attempts have been made to assess personal exposure to household appliances which are regularly used both in the UK $(50 \mathrm{~Hz})$ and North America $(60 \mathrm{~Hz})$, as noted in Table 1 and Table 2 respectively.

Preece et al. (1997) assessed the magnetic field strength $(40-800 \mathrm{~Hz})$ of 806 domestic appliances within 50 UK homes (Table 1) using the Emdex II (electric and magnetic field digital exposure system) to measure the appliance-generated magnetic field. Upon activation of the appliance, measurements were recorded every 3 seconds for 30 seconds at 5, 30, 60 and 100 centimetres from the appliance surface. Similarly, Farag et al. (1998) assessed the magnetic field strength of domestic appliances at various distances up to 120 centimetres in 254 homes (Table 2).

The relatively large magnetic fields experienced when in close proximity to domestic appliances have in fact been hypothesised to contribute to an increased risk of hormone-dependent cancers and in particular breast cancer (Stevens, 1987). While studies have provided a weak yet significant link between the use of electric razors and breast cancer (odd ratio, $\mathrm{OR}=1.7,95 \%, \mathrm{Cl}$ : 1.1-2.8) (Zheng et al., 2000b), and meningioma ( $\mathrm{OR}=10.9,95 \%, \mathrm{Cl}: 2.3-50)$ (Kleinerman et al., 2005), there has been no association between breast cancer and the use of hair dryers, curling irons, vacuum cleaners or irons (Zheng et al., 2000b). Moreover, little evidence has been provided of an association between brain cancer and either curling irons or microwave ovens (Kleinerman et al., 2005).

Electric blankets however represent a further appliance that has been extensively studied in relation to an increased risk in potential adverse health effects (Savitz et al., 1990; Abel et al., 2007). Comparatively, the magnetic fields emitted by electric blankets are weaker than those of hair dryers and electric razors with residential measurements at a distance of 10 centimetres recording flux densities between 0.1$2.2 \mu \mathrm{T}$ (Wilson et al., 1996; Hatch et al., 1998; Lee et al., 2000). However, the exposure from appliances is related to the duration of the dose alongside the distance from the source and, unlike electric blankets, none of the appliances indicated in Tables 1 and 2 are employed over an extended period of time. In contrast, Preston-Martin et al. (1996) reported that $83 \%$ of mothers and $84 \%$ of children left the blanket on for the 
whole night. Therefore, the use of electric blankets represents a sustained source of magnetic field exposure that is within close proximity to the body and thus may provide vital understanding into the association of exposure to magnetic fields with such diseases.

Table 1. The magnetic fields from $50 \mathrm{~Hz}$ supplied household appliances

\begin{tabular}{|c|c|c|c|c|}
\hline \multirow[t]{2}{*}{ Appliance } & \multirow{2}{*}{$\begin{array}{l}\text { Number of } \\
\text { appliances } \\
\text { measured }\end{array}$} & \multicolumn{3}{|c|}{$\begin{array}{l}\text { Magnetic field at specified distance from the exterior } \\
\text { of appliance }(\mu \mathrm{T})\end{array}$} \\
\hline & & $5 \mathrm{~cm} \pm$ S.D & $50 \mathrm{~cm} \pm S . D$ & $100 \mathrm{~cm} \pm S . D$ \\
\hline Television & 73 & $2.69 \pm 1.08$ & $0.26 \pm 0.11$ & $0.07 \pm 0.04$ \\
\hline Kettle & 49 & $2.82 \pm 1.51$ & $0.05 \pm 0.06$ & $0.01 \pm 0.02$ \\
\hline Video recorder & 42 & $0.57 \pm 0.52$ & $0.06 \pm 0.05$ & $0.02 \pm 0.02$ \\
\hline Vacuum cleaner & 42 & $39.53 \pm 74.58$ & $0.78 \pm 0.74$ & $0.16 \pm 0.12$ \\
\hline Hair-dryer & 39 & $17.44 \pm 15.56$ & $0.12 \pm 0.10$ & $0.02 \pm 0.02$ \\
\hline Microwave oven & 34 & $27.25 \pm 16.74$ & $1.66 \pm 0.63$ & $0.37 \pm 0.14$ \\
\hline Washing machine & 34 & $7.73 \pm 7.03$ & $0.96 \pm 0.56$ & $0.27 \pm 0.14$ \\
\hline Iron & 33 & $1.84 \pm 1.21$ & $0.03 \pm 0.02$ & $0.01 \pm 0.00$ \\
\hline Clock radio & 32 & $2.34 \pm 1.96$ & $0.05 \pm 0.05$ & $0.01 \pm 0.01$ \\
\hline Hi-fi system & 30 & $1.56 \pm 4.29$ & $0.08 \pm 0.14$ & $0.02 \pm 0.03$ \\
\hline Toaster & 29 & $5.06 \pm 2.71$ & $0.09 \pm 0.08$ & $0.02 \pm 0.02$ \\
\hline Central heating boiler & 26 & $7.37 \pm 10.10$ & $0.27 \pm 0.26$ & $0.06 \pm 0.05$ \\
\hline Fridge / freezer & 23 & $0.21 \pm 0.14$ & $0.05 \pm 0.03$ & $0.02 \pm 0.01$ \\
\hline Cooker & 18 & $2.27 \pm 1.33$ & $0.21 \pm 0.15$ & $0.06 \pm 0.04$ \\
\hline Dishwasher & 13 & $5.93 \pm 4.99$ & $0.80 \pm 0.46$ & $0.23 \pm 0.13$ \\
\hline Freezer & 13 & $0.42 \pm 0.87$ & $0.04 \pm 0.02$ & $0.01 \pm 0.01$ \\
\hline Oven & 13 & $1.79 \pm 0.89$ & $0.39 \pm 0.23$ & $0.13 \pm 0.09$ \\
\hline Electric shower & 12 & $30.82 \pm 35.04$ & $0.44 \pm 0.75$ & $0.11 \pm 0.25$ \\
\hline Food processor & 10 & $12.84 \pm 12.84$ & $0.23 \pm 0.23$ & $0.04 \pm 0.04$ \\
\hline Extractor fan & 9 & $45.18 \pm 107.96$ & $0.50 \pm 0.93$ & $0.08 \pm 0.14$ \\
\hline Hand blender & 8 & $76.75 \pm 87.09$ & $0.97 \pm 1.05$ & $0.15 \pm 0.16$ \\
\hline Tumble dryer & 7 & $3.93 \pm 5.45$ & $0.34 \pm 0.42$ & $0.10 \pm 0.10$ \\
\hline Computer & 6 & $1.82 \pm 1.96$ & $0.14 \pm 0.07$ & $0.04 \pm 0.02$ \\
\hline
\end{tabular}

The arithmetic means of calculated broadband magnetic fields at 5, 50 and 100 centimetres $(\mathrm{cm})$ from a selected number of household appliances using a $50 \mathrm{~Hz}$ power system supply. Adapted from Preece et al., (1997). 
Table 2. The magnetic fields from $60 \mathrm{~Hz}$ supplied household appliances

\begin{tabular}{|c|c|c|c|c|}
\hline \multirow{2}{*}{ Appliance } & \multirow{2}{*}{$\begin{array}{l}\text { Number of } \\
\text { appliances } \\
\text { measured }\end{array}$} & \multicolumn{3}{|c|}{ Exposure $(\mu \mathrm{T})$ at $10 \mathrm{~cm}$} \\
\hline & & Minimum & Median & Maximum \\
\hline Dishwasher & 12 & 0.55 & 2.08 & 2.2 \\
\hline Tumble dryer & 32 & 0.41 & 3 & 16.5 \\
\hline Electric shaver & 69 & 1 & 2 & 13 \\
\hline Hair-dryer & 117 & 0.3 & 1.5 & 19 \\
\hline Microwave oven & 137 & 0.26 & 7 & 44.2 \\
\hline Refrigerator & 225 & 0.065 & 0.66 & 3.2 \\
\hline Washing machine & 46 & 0.2 & 0.69 & 1.4 \\
\hline \multirow[t]{2}{*}{ Oven } & 230 & 0.12 & 1.21 & 5.05 \\
\hline & & \multicolumn{3}{|c|}{ Exposure $(\mu \mathrm{T})$ at $30 \mathrm{~cm}$} \\
\hline \multirow[t]{2}{*}{ Vacuum cleaner } & 34 & 1.4 & 5.1 & 20.5 \\
\hline & & \multicolumn{3}{|c|}{ Exposure $(\mu \mathrm{T})$ at $50 \mathrm{~cm}$} \\
\hline Air conditioner & 27 & 0.025 & 0.16 & 0.82 \\
\hline Blender & 27 & 0.21 & 0.22 & 0.7 \\
\hline Electric blower & 16 & 0.018 & 0.13 & 1.05 \\
\hline $\begin{array}{l}\text { Electric can } \\
\text { opener }\end{array}$ & 27 & 0.92 & 1.45 & 6.2 \\
\hline Ceiling fan & 24 & 0.02 & 0.15 & 1.2 \\
\hline Coffee maker & 20 & 0.05 & 0.095 & 0.16 \\
\hline Iron & 60 & 0.052 & 0.14 & 0.3 \\
\hline Non-ceiling fan & 21 & 0.015 & 0.26 & 3.2 \\
\hline Food processor & 24 & 0.35 & 1.2 & 4 \\
\hline Toaster & 18 & 0.042 & 0.21 & 0.51 \\
\hline Oven & 230 & 0.025 & & 1.45 \\
\hline
\end{tabular}

The minimum, median and maximum magnetic flux density at 10, 30 and $50 \mathrm{~cm}$ from a selected number of household appliances using a $60 \mathrm{~Hz}$ power system supply. Values courtesy of Farag et al., (1998).

Finally, the transmission of electrical power through high-voltage transmission and distribution lines involves the flow of electrical current and ultimately the production of magnetic fields. As a result, exposure to stray fields associated with the generation and transmission of electricity has been a regular occurrence for nearly a century (Jackson, 1992).

\subsection{Power Lines}

The transportation of electricity from the power station into residences occurs via a wide range of voltages and hence currents, which are determined by the distance of transmission (Jackson, 1992). The magnetic field produced and ultimately the population's exposure is related to the properties of the transmission/distribution line. 
Long distance transmission is conducted by 500 kilovolts $(\mathrm{kV})$ and 275/400 kV highvoltage transmission lines in the USA and UK respectively. Once within a local region, these voltages are stepped down by transformers and $132 \mathrm{kV}, 33 \mathrm{kV}$ and $11 \mathrm{kV}$ distribution lines carry the electricity into the neighbourhoods, where neighbourhood transformers further step down the voltage to residential delivery via domestic service lines (Kaune, 1993; Merchant et al., 1994). In the UK, as with most of Europe, electricity is delivered into homes at $220-240$ volts, in comparison to North America $(110 \mathrm{~V})$, therefore the currents needed to provide the same power are lower in the UK and Europe (Repacholi, 2012). Similar to electrical appliances, the strongest magnetic fields are in the immediate vicinity of the source (directly beneath the power line) and diminish in relation to distance (Table 3) so that at approximately 100 metres they are typically equivalent to background fields (Draper et al., 2005; Kroll et al., 2010).

Table 3. UK and USA average power line exposure

\begin{tabular}{|c|c|c|}
\hline \multicolumn{2}{|l|}{ Source of magnetic fields (USA) } & \multirow{2}{*}{$\begin{array}{l}\text { Field strength } \\
(\mu \mathrm{T}) \\
8.7\end{array}$} \\
\hline 500 kV high-voltage & Typical average under line & \\
\hline transmission line & Typical average $20 \mathrm{~m}$ from centre of line & 2.9 \\
\hline \multirow{2}{*}{$\begin{array}{l}24 \mathrm{kV} \text { overhead and } \\
\text { underground distribution } \\
\text { lines }\end{array}$} & Typical maximum beneath and over lines & $4-7$ \\
\hline & Typical average beneath and over lines & $1-2$ \\
\hline $\begin{array}{l}\text { Mean exposure to a typical } \\
\text { person from all sources of } \\
\text { magnetic fields over a } 24 \text { hour } \\
\text { period }\end{array}$ & & $<0.05$ \\
\hline \multicolumn{2}{|l|}{ Source of magnetic fields (UK) } & $\begin{array}{l}\text { Field strength } \\
(\mu \mathrm{T})\end{array}$ \\
\hline \multirow{2}{*}{$\begin{array}{l}275 / 400 \mathrm{kV} \text { high-voltage } \\
\text { transmission line }\end{array}$} & Typical average under line & 5.7 \\
\hline & Typical average $20 \mathrm{~m}$ from centre of line & 2 \\
\hline \multirow[t]{2}{*}{132 kV distribution line } & Typical average under line & $0.5-2$ \\
\hline & Typical average $25 \mathrm{~m}$ from centre of line & $0.05-0.2$ \\
\hline \multirow[t]{2}{*}{$33 \mathrm{kV}$ distribution lines } & Typical average under line & 1.5 \\
\hline & Typical average $20 \mathrm{~m}$ from centre of line & 0.1 \\
\hline \multirow[t]{2}{*}{$400 \mathrm{~V}$ service line } & Typical average under line & $0.143-0.215$ \\
\hline & Typical average $25 \mathrm{~m}$ from centre of line & $0.039-0.041$ \\
\hline $\begin{array}{l}\text { Average exposure to a typical } \\
\text { person from all sources of } \\
\text { magnetic fields over a } 24 \text { hour } \\
\text { period }\end{array}$ & & $<0.11$ \\
\hline
\end{tabular}

Typical average exposures to sources of power frequency magnetic fields in the USA, (values courtesy of (Repacholi, 2012) and UK, (EMFs.info, 2015). Adapted from (Repacholi, 2012). 
It must be noted that while residing in close vicinity of high-voltage power lines will lead to high levels of electric and magnetic fields' exposure, only a small percentage of the population own homes within these areas. In the UK $0.07 \%$ of homes are within 50 metres of high-voltage transmission lines, while $0.21 \%$ lies within 100 metres. These figures correlate with those provided in the USA where approximately $1.1 \%$ of residences are within 40 metres of high-voltage power lines (EMFs.info, 2015).

Moreover, as is illustrated by Table 3, even directly under a typical high-voltage transmission line the average ELF-MF exposure is less than $5 \%$ of the International Commission on Non-Ionising Radiation Protection (ICNIRP) guideline limits (Chapter 1.1.4), (International Commission on Non-Ionizing Radiation Protection, 2010). Taken together, these percentages are particularly pertinent following the publication of several studies associating ELF-MF exposure of residences closer to high-voltage power lines with an increased risk of cancer (Coleman et al., 1989; Feychting \& Ahlbom, 1993; Draper et al., 2005). On the other hand, conflicting studies have similarly been published in which residences in close proximity to high-voltage power lines present no significant association with an increased onset of cancer (Olsen et al., 1993; Kleinerman et al., 2000; UK Childhood Cancer Study Investigators, 2000; Pedersen et al., 2014). A further study noted a significant risk of leukaemia attached up to the 1980 's, though from thereon in, risk declined to insignificance throughout subsequent decades (Bunch et al., 2014). Nonetheless, the indication of a potential elevated risk ensures that ELF-MF falls within the scope of the ICNIRP, an organisation established in 1992 to develop universally appropriate guidelines detailing exposure limits to potentially hazardous forms of non-ionising radiation.

\subsubsection{Exposure Guidelines}

It was following a comprehensive review of the published literature, and in an attempt to safeguard against any potential adverse health effects relating to both residential and occupational ELF-MF exposure, that the initial ELF-MF exposure guidelines were established in 1998. Within the $100 \mathrm{kHz}$ frequency range, which includes power frequencies $50 \mathrm{~Hz}$ and $60 \mathrm{~Hz}$, reference limits of $500 \mu \mathrm{T}$ and $100 \mu \mathrm{T}$ were set for occupational workers and the general population respectively (International Commission on Non-lonizing Radiation Protection, 1998). A safety factor of five was 
applied to occupational exposure to represent workers' chronic exposure to stronger fields (International Commission on Non-lonizing Radiation Protection, 1998).

However, several publications further linking ELF-MF exposure to an increased risk of cancer, in addition to numerous biological and genotoxic effects (IARC Working Group on the Evaluation of Carcinogenic Risks to Humans, 2002; WHO, 2007), the ICNIRP reconsidered their guideline limits in 2010 and raised them to $200 \mu \mathrm{T}$ and $1 \mathrm{mT}$ for residential and occupational exposure respectively (International Commission on NonIonizing Radiation Protection, 2010). Ultimately, the ICNIRP reasoned that in an overall absence of consistent, replicated experimental research coupled to a lack of a defined causal relationship, meant that any potential observed effect would not be confronted in such basic exposure restrictions.

However, an issue still remains in respect to these guidelines in that a considerable number of epidemiologic studies, alongside several pooled and meta-analysis studies have regularly indicated that chronic exposure to $50 / 60 \mathrm{~Hz}$ magnetic fields many times lower $(0.4 \mu \mathrm{T})$ is associated with an increased risk of numerous health problems, with probably the most consistent link being to an association with childhood leukaemia (Ahlbom et al., 2001). Indeed, the initial link to childhood leukaemia was reported in 1979 when Wertheimer and Leeper published their ground-breaking study in which they developed and employed a non-invasive method as a surrogate of ELF-MF exposure assessment, known as 'wire codes' (Wertheimer \& Leeper, 1979). Residences were classified based on the observable characteristics of the electrical cables in close proximity, therefore the distance, size and the type of cable were used to categorise homes as possessing either a high-current configuration (HCC) or a low-current configuration (LCC) (Table 4). Ultimately, homes classified as HCC were presumed to be susceptible to stronger background fields than those of LCC. Using this surrogate to indirectly calculate the potential current flow, Wertheimer and Leeper (1979) performed a case-control study within the greater Denver area of Colorado during 1976 to 1977 . A total of 344 cases were selected through a stringent two-step process of individuals diagnosed with cancer in Colorado before the age of 19 and between the years of 1950 to 1973, who also presented a Colorado birth certificate. The assignment of matched controls however is rather complicated and inadequately presented in the 
literature (Lagiou et al., 2002). Though a simplified explanation presented in the IARC summary (2002), indicates the matched-controls were assigned through the possession of a Denver-area birth certificate, alongside lists of births within the Colorado area during the time period of 1946-1973. Although relative risks were not presented in their study, Wertheimer and Leeper reported a statistically significant increase in leukaemia mortality among children who lived in close proximity to cables representing high current configurations. Subsequent re-analyses of the data have indicated a three-fold increase in childhood leukaemia (Kheifets et al., 1997; LacyHulbert et al., 1998).

\subsection{Childhood Leukaemia}

The elevated risk to children is especially pertinent as the role of ELF-MF becomes more prominent in modern society because, although cases of childhood cancers are sparse, of those recorded approximately one third relate to childhood leukaemia (CL), making it the most common type of childhood cancer (Linet et al., 1999). Leukaemia is a cancer that arises in the hematopoietic tissue, i.e. bone marrow before entering the blood, occurring as a result of chromosomal alterations and mutations that disturb the normal differentiation of blood progenitor cells into either lymphoid or myeloid progenitor cells (Chiaretti et al., 2014). Subsequently, leukaemia is classified according to the cell of origin, as well as its clinical course (acute or chronic) (Brain et al., 2003). As such, the major morphologic types are acute lymphoblastic leukaemia (ALL) and acute myeloid leukaemia (AML), which refers to cancer of lymphoid or myeloid progenitor cells respectively. Acute lymphoblastic leukaemia is the most common form of $\mathrm{CL}$, accounting for around $78 \%$ of the diagnosed cases, while $\mathrm{AML}$ accounts for the majority of the remaining diagnoses (Belson et al., 2007). The age of onset for ALL follows a bimodal distribution whereby cases are normally reported up to the age of 15 years (Inaba et al., 2013), with the peak incidence occurring in children aged between 2 and 5 years (Linet \& Devesa, 1991; Greaves \& Wiemels, 2003; Pui et al., 2008). There then follows a steady increase in prevalence throughout life. The risk of $A M L$ on the other hand is highest within the first two years immediately after birth before decreasing thereafter (Gurney et al., 1995). Although the knowledge of genetic changes in $\mathrm{CL}$ has improved significantly over the years, the precise aetiology of 
childhood leukaemia remains largely unknown. Less than $5 \%$ of cases of ALL are connected with inherited genetic syndromes or ionising radiation (Pui et al., 2008), which has led researchers to hypothesise that development of the disease results from a combination of factors involving genetic susceptibility, environmental factors and chance (Greaves, 2002). This has led to the development of a 'two-hit' model (Figure 2), whereby the onset and progression of leukaemia consists of a multistep process (Greaves, 2006). The general model assumes an initial somatic hit, occurring in utero in a foetal hematopoietic cell, probably as a result of chromosome translocations. This initial hit is believed to be a common event that results in the formation and proliferation of a pre-leukaemic clone (Greaves, 1999). Yet, the reasonably low prevalence of the disease illustrates at least a two-stage pathogenesis in which the conversion of the pre-leukaemic clone into the disease requires either an additional pre-natal and/or post-natal genetic or environmental event (Eden, 2010). Such events are rare: only $\sim 1 \%$ of individuals possessing pre-leukaemic clones will get an additional genetic hit to create overt leukaemia, thus acting as a rate-determining step in the progression of the disease (Greaves \& Wiemels, 2003).

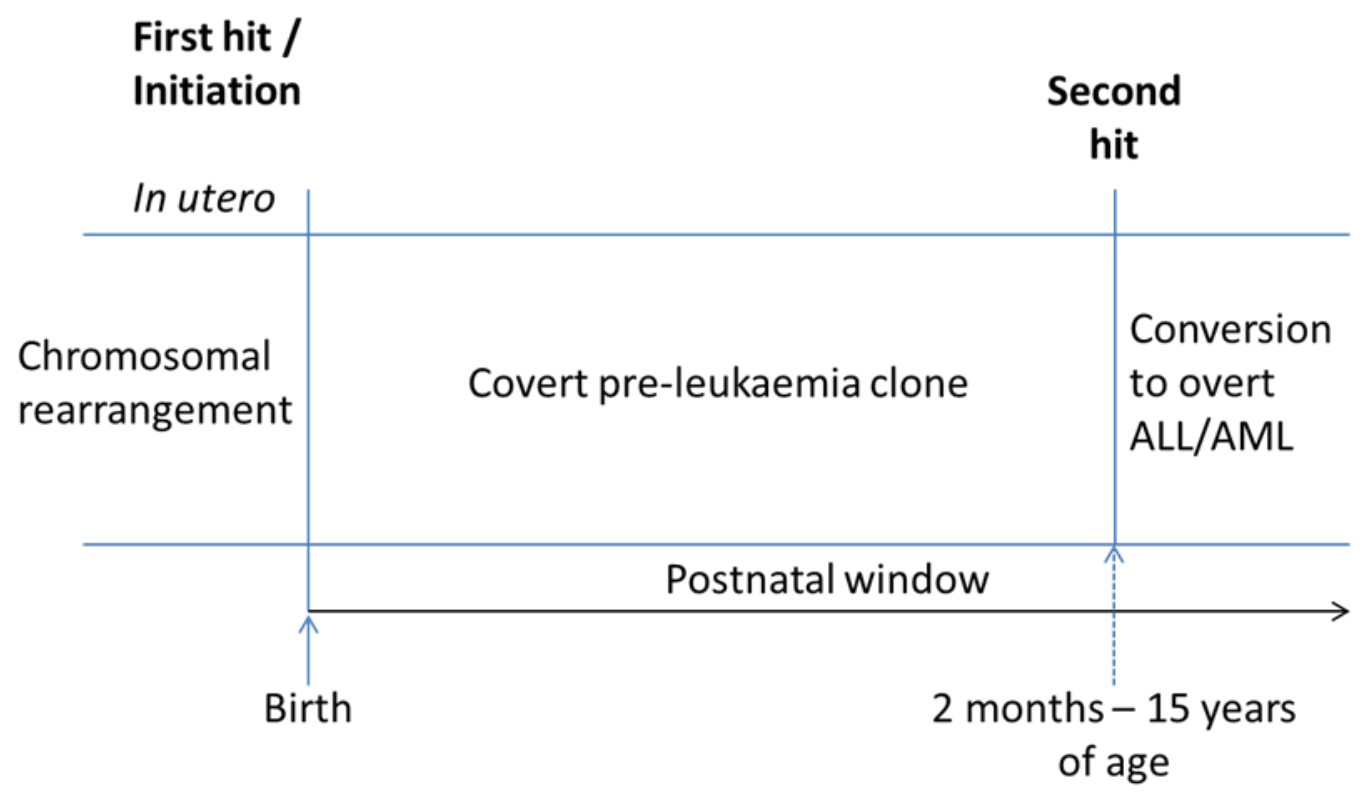

Figure 2. A simplified Two-Hit Model for potential CL onset. Illustrating the crucial events hypothetically required to produce the major morphologic types of childhood leukaemia. Adapted from (Greaves, 2006). 
If indeed the two hit model requires an environmental interaction, in spite of the vast number of epidemiologic studies that have researched a multitude of environmental agents in the general population, ionising radiation remains as the only widely accepted environmental causative factor, but still only accounts for a very limited amount of CL cases (Preston et al., 1994; Doll \& Wakeford, 1997). Other suspected risk factors include exposure to environmental agents such as benzene, the human T-cell virus and genetic disorders such as Down's syndrome (reviewed in (Belson et al., 2007; Eden, 2010; Mezei et al., 2014)). Finally, owing to the seminal study by Wertheimer and Leeper (1979), ELF-MF is also hypothesised as a potential environmental exposure responsible for a causative link to $\mathrm{CL}$.

Despite the above, the results of subsequent epidemiologic studies conducted over the past three decades have often presented contradictory and inconclusive evidence of a causal relationship between ELF-MF exposure and an increased risk of cancer. A prime example of such inconsistency between studies is highlighted in a study conducted by Fulton et al. (1980), where attempts to replicate the findings of Wertheimer and Leeper (1979) indicating a relationship between childhood leukaemia and electric power line configurations in Rhode Island $(\mathrm{OR}=1.09,95 \% \mathrm{Cl}: 0.7-1.6)$ proved unsuccessful, despite implementing the same wire classification analysis. Such underlying contradictions present within epidemiological studies are predominantly due to the ubiquitous nature of ELF-MFs in the environment, which has consequently resulted in the assessment of ELF-MF becoming especially problematic. Indeed, in most studies it is more important to assign the residence to the correct classification in an attempt to reduce the chance of misclassification rather than extract an exact exposure measurement value (Kheifets \& Oksuzyan, 2008). In fact, throughout the years and hence epidemiologic studies, there has been a plethora of exposure matrixes used which generated both positive and negative correlations to childhood leukaemia.

\subsection{Epidemiologic Studies}

\subsubsection{Residential Exposure}

The most basic and simple of exposure assessments is to measure the distance between the residence and source as it does not take into consideration multiple sources of exposure. Firstly, Coleman et al. (1989) assessed the potential relationship 
of indirect residential exposure to power frequency magnetic fields from power lines and transformer substations through distance. The authors state that the risk of leukaemia relative to cancer controls when residing within 100 metres was 1.45 (95\% $\mathrm{Cl}: 0.54-3.88)$; within 50 metres the relative risk (RR) increased to 2.0 but the confidence intervals also expanded (95\% Cl: 0.4-9.0) while residences within 25 metres carried a risk of 1.3 (95\% Cl: 0.8-2.0) (Coleman et al., 1989). Although, as previously mentioned (Chapter 1.1.3.2.3), approximately only $0.21 \%$ of households lie within 100 metres of high-voltage transmission lines in the UK (EMFs.info, 2015). Moreover the authors reported that only $6 \%$ of the 771 case subjects lived within 100 metres of an overhead power line while $40 \%$ lived within 100 metres of a substation ( $O R=0.99$ ) (Coleman et al., 1989). Similarly, subsequent studies have also failed to report a significantly raised odds ratio between childhood cancer and proximity to overhead power lines (Myers et al., 1990; Verkasalo et al., 1993). Alternatively, some evidence of increased risk of all cancers was observed by Olsen and co-authors for calculated exposures of more than $0.4 \mu \mathrm{T}$, but not for leukaemia when taken separately (Olsen et al., 1993). The lack of significantly raised odds ratios does not signify a lack of true association, but more so that too few addresses for cases and controls within their study had background levels of magnetic fields exceeding $0.01 \mu \mathrm{T}$ (Myers et al., 1990). Thus, in an attempt to record more accurate exposures, the 'wire code' classification system was further developed, firstly to include four (Wertheimer \& Leeper, 1982) and later five (Savitz et al., 1988) sub-classes of home exposure (Table 4). 
Table 4. Wire code assessment categories

\begin{tabular}{|c|c|c|c|c|c|c|}
\hline \multirow{3}{*}{$\begin{array}{l}\text { Wire code } \\
\text { category }\end{array}$} & \multicolumn{6}{|c|}{ Type of external power line \& distance to residence } \\
\hline & \multirow{2}{*}{$\begin{array}{l}\text { Transmission } \\
\text { line }\end{array}$} & \multicolumn{3}{|c|}{3 phase primary distribution } & \multicolumn{2}{|c|}{ Secondary distribution } \\
\hline & & $\geq 2$ & Thick & Thin & $1^{\text {st }}$ span & $\geq 2^{\text {nd }}$ span \\
\hline \multicolumn{7}{|l|}{ HCC } \\
\hline VHCC & $<15 \mathrm{~m}$ & $<15 \mathrm{~m}$ & $<15 \mathrm{~m}$ & $<7.5 \mathrm{~m}$ & & \\
\hline $\mathrm{OHCC}$ & $<40 \mathrm{~m}$ & $<40 \mathrm{~m}$ & $<40 \mathrm{~m}$ & $<20 \mathrm{~m}$ & $<15 \mathrm{~m}$ & \\
\hline \multicolumn{7}{|l|}{ LCC } \\
\hline OLCC & & & & $<40 \mathrm{~m}$ & $<40 \mathrm{~m}$ & $<40 \mathrm{~m}$ \\
\hline VLCC & \multicolumn{6}{|c|}{ None of the above and secondary service overhead } \\
\hline UG & \multicolumn{6}{|c|}{ None of the above and secondary service underground } \\
\hline
\end{tabular}

Representative of the specifications of the wire codes used as a surrogate index for residential magnetic field exposure assessment. High-current configuration (HCC) and low-current configurations were used in Wertheimer \& Leeper, (1979). Subsequent studies expanded the indices to include very high-current configuration (VHCCs), ordinary high-current configuration (OHCCs), ordinary low-current configuration (OLCCs), very low-current configuration (VLCC) and underground cables (UG). Adapted from Kheifets et al., (1997).

\subsubsection{Wire Code Studies}

Using the modified wire code categories, Savitz et al. (1988) again performed a casecontrol study in Denver. The odds ratios presented were generally consistent with the corrected odds ratios of Wertheimer and Leeper (1979). However Savitz and coauthors (1988) concluded that their study did not indicate a series of replicated positive studies but rather a body of literature of suggestive, yet inconclusive, results when taken both as an aggregate and individually. Re-analysis of the data using modified wire codes (high-, medium- and low wire codes), further facilitated the production of more precise odds ratio providing an association for leukaemia, 2.9 (Cl: 1.5-5.5) (Savitz \& Kaune, 1993). Consistent with these earlier studies, London et al. (1991) also observed a statistically significant association between very high exposure classifications and an increased risk for leukaemia. However, in contrast to these earlier studies, McBride et al. (1999) provided little support for an association between ELF-MF exposure and an increased risk of $\mathrm{CL}$, publishing results portraying a statistically non-significant risk associated with exposure pertaining to high wire codes. Similarly, Linet et al. (1997) found no association between residences classified within the highest wire code category in either the modified Wertheimer and Leeper category (Table 4) or the three code's surrogate employed by Savitz and Kaune (1993). The lack 
of an association was observed in both the main residence and the one the pregnant mother had resided in (Linet et al., 1997).

\subsubsection{Field Measurement Studies}

While the use of wire codes as a surrogate of exposure to determine predicted magnetic field levels eliminates the prospect of socioeconomic bias, no account of exposure from within the home is taken (Kheifets et al., 1997). The development of magnetic field meters further enhanced the accuracy of exposure assessment, allowing for direct measurements of magnetic fields to be undertaken in homes and over extended periods. Importantly, the use of spot measurements does not provide any reference to variability throughout the day or even seasonal variability unless measurements are constantly repeated (Kheifets \& Oksuzyan, 2008). A solution is to record measurements over a longer period. These can be either undertaken as personal or location-based measurements, and normally take place over a 24-48 hour period.

Tomenius (1986) conducted the first European study in relation to childhood leukaemia and ELF-MF exposure. The analysis was based on residences and their proximity to different types of electrical installations, alongside spot measurements that were taken outside the entrance doors. Statistically significant odds ratios in children exposed to magnetic fields $\geq 0.3 \mu \mathrm{T}$ were presented for all tumours combined $(O R=2.1)$, notably however not for leukaemia $(O R=0.3)$. Similarly, Savitz et al. (1988), reported that measured magnetic fields under low power use conditions $(\geq 0.2 \mu \mathrm{T})$ when compared to a cut-off of $<0.2 \mu \mathrm{T}$, had a modest but statistically insignificant association with total cancer incidence ( $\mathrm{OR}=1.4 ; \mathrm{Cl}: 0.63-2.9)$, observing odds ratios of 1.9 (Cl: 0.67-5.6) for leukaemia. Furthermore, the study conducted by London et al. (1991), which was the first to perform long term-exposure (24-hour) measurements of ELF-MF, in addition to spot (single time point) measurements also observed a nonsignificant elevated odds ratio in association to childhood leukaemia risk (OR $=1.69$, $95 \% \mathrm{Cl}: 0.71-4.00)$. Subsequent studies have also found a lack of significant risk of childhood leukaemia (Feychting \& Ahlbom, 1993; Coghill et al., 1996; Michaelis et al., 1998; McBride et al., 1999). Equally, there were no associations in the UKCC study (1999), where adjusted odds ratios of 0.92 (95\% Cl: 0.47-1.79) for acute lymphoblastic 
leukaemia, $0.90(0.49-1.63)$ for all leukaemia and $0.46(0.11-1.86)$ for children with mean exposures of $>0.2 \mu \mathrm{T}$ compared with children with mean exposures of $<0.1 \mu \mathrm{T}$ were published (UK Childhood Cancer Study Investigators, 1999). There were however suggestions of a positive association between $\mathrm{CL}$ and ELF-MF $\geq 0.2 \mu \mathrm{T}$ (Dockerty et al., 1998), although the study only possessed five cases and one control within this exposure group, and the confidence intervals produced were too wide to form a viable conclusion (Cl: 1.1-224). Additionally, data produced by Linet et al. (1997) suggest an increase in risk at fields $\geq 0.3 \mu \mathrm{T}$, but again the number of cases in which this exposure category was represented was small and, in contrast to Dockerty et al. (1998), no association was witnessed at $\geq 0.2 \mu \mathrm{T}$. If an effect does in fact exist below $0.2 \mu \mathrm{T}$, then it is likely too small to reach consensus in epidemiological studies alone (Greenland et al., 2000).

In addition to studies recording spot and long-term measurements, some used the historical loads as recorded by power companies at the time of diagnosis to calculate magnetic field exposure retrospectively (Feychting \& Ahlbom, 1993). The use of historical loads to calculate magnetic fields provided some evidence of an increased risk of $\mathrm{CL}$ for exposures $>0.3 \mu \mathrm{T}(\mathrm{OR}=3.8,95 \% \mathrm{Cl}$ : 1.4-9.3) (Feychting \& Ahlbom, 1993). Likewise, a meta-analysis in 1998 reported a weak elevated risk of leukaemia for children exposed to residential magnetic fields and a positive association between childhood leukaemia and calculated historic fields $\geq 0.2 \mu \mathrm{T}$ (OR $=1.90,95 \% \mathrm{Cl}$ : 1.073.39) (Wartenberg, 1998). However, Tynes and Haldorsen (1997) failed to reproduce such findings, presenting no association between childhood cancer, including leukaemia, and residences near power lines, based on calculations from power line distance and records of load. While, in a further meta-analysis study comprised of risk estimates from 14 case-control studies and one cohort study, no association between childhood leukaemia and calculated magnetic fields from wiring configuration codes could be substantiated, since the heterogeneity amongst individual studies was found to be statistically significant (Angelillo \& Villari, 1999).

If follows that the considerable variation between studies illustrated throughout this chapter could be partially attributed to the numerous methods of exposure assessment that were employed, but it must also be noted that most studies involved 
small numbers of cases within the top exposure categories. However, the organisation and review of the existing data may yet provide important insights into the consistency of the results. These analyses have taken form as two separate entities, pooled analysis studies and meta-analyses.

\subsubsection{Meta-\& Pooled Analysis Studies}

Pooled analysis lays the benchmark for synthesising results from multiple studies. Raw data are collected from previous studies which can then be compared free from artefacts introduced by analytical differences for the gleaning of statistically more stable results (Kheifets et al., 2006; Kheifets et al., 2010). However, despite their strengths, results derived from pooled analyses are still prone to the same biases as the original studies were subjected to. Thus in an attempt to provide a more reliable evaluation of inter-study differences in results, two pooled analyses based on studies up to 1999 were published in 2000 (Ahlbom et al., 2000; Greenland et al., 2000). The first was conducted by a team funded through the National Institute of Environmental Health Sciences. Combining studies that used magnetic field measures as their primary analysis, with those that used wire codes, Greenland and co-authors (2000) derived exposure summary estimates from 12 studies based on 2656 cases and 7084 controls. Of those 12, only two (London et al., 1991; Linet et al., 1997) provided more than four cases exposed to $\geq 0.4 \mu \mathrm{T}$, hence for categorical magnetic field analysis these cases were combined into a single category of exposures $>0.3 \mu \mathrm{T}$. A positive association was concluded to exist between ELF-MF and childhood leukaemia when comparing $>0.3 \mu \mathrm{T}$ to exposures $<0.1 \mu \mathrm{T}(\mathrm{OR}=1.68,95 \% \mathrm{Cl}: 1.23-2.31)$ (Greenland et al., 2000). Likewise, a further pooled analysis study showed a two-fold increase in risk of childhood leukaemia during the year prior to diagnosis with exposures $\geq 0.4 \mu \mathrm{T}$ (RR 2.00, 95\% Cl: 1.27-3.13) when compared with a reference level exposure of $<0.1 \mu \mathrm{T}$ (Ahlbom et al., 2000). While it is highlighted that exposures $>0.4 \mu \mathrm{T}$ signify a doubling in risk of childhood leukaemia it must also be noted that this exposure category was only representative of $0.8 \%$ of subjects ( 44 cases and 62 controls). A more recent pooled analysis based on primary data from epidemiological studies on residential magnetic fields and childhood leukaemia published post 2000, found a statistical non-significant risk of leukaemia associated with increased exposure $(\geq 0.3 \mu$ T when compared to $<0.1$ $\mu \mathrm{T}, \mathrm{OR}=1.44,95 \% \mathrm{Cl}: 0.88-2.36$ ) (Kheifets et al., 2010). However, the overall number 
of cases and controls were once more small with only 26 cases and 50 controls present within the $\geq 0.3 \mu \mathrm{T}$ category. Indeed this led the authors to conclude that, though there was a lack of statistical significance, their results were in line with previous pooled studies. Furthermore, that their lack of significant data does not alter the previous assessment that magnetic fields are possibly carcinogenic (Kheifets et al., 2010). More recently still, Zhao et al. (2014) aimed to further assess the link between childhood leukaemia and magnetic field exposure in a meta-analysis based on circa 25,000 individuals. A meta-analysis, similar to pooled analysis, is a statistical method extensively used in biomedical research, which is specifically useful in providing a more reliable outcome when data from different studies are inconclusive (Lagiou et al., 2002). A single study, taken separately may be either too small or limited in design to generate a conclusive outcome. Thus, by combining the data from similar, previously published studies, to provide a single summary risk estimate, a more reliable representation of the evidence is presented (Vijayalaxmi \& Prihoda, 2009). A total of 11,699 cases and 13,194 controls from nine studies between the years of 1997 to 2013 were included under strict selection criteria. Again using $<0.1 \mu \mathrm{T}$ as the reference, a statistically significant association was exhibited between both total childhood leukaemia and more specifically acute lymphocytic leukaemia and magnetic field intensity of $\geq 0.4 \mu \mathrm{T}$ and, $\mathrm{OR}=1.57,95 \% \mathrm{Cl}: 1.03-2.40$ and $\mathrm{OR}=2.43,95 \% \mathrm{Cl}: 1.30-$ 4.55, respectively (Zhao et al., 2014). While this indicates some concordance between studies and potentially identifies a very specific exposure limit associated with damage, only a few residences registered average spot- or long-term measurements $>0.3 \mu \mathrm{T}(1-4 \%)$ and even fewer (1-2\%) >0.4 $\mu \mathrm{T}$ (Ahlbom et al., 2000; Greenland et al., 2000). Considering the small amount of individuals who are in fact exposed to ELF-MFs greater than $0.4 \mu \mathrm{T}$, it follows that an estimate of between one and two additional cases (per 500) would arise as a result of artificial ELF-exposure (UK Childhood Cancer Study Investigators, 1999).

While the omnipresence of background fields occupy the majority of concern, an abundance of studies have been conducted in order to analyse the relationship between ELF-MF exposure from electric blankets and the incidence of several types of cancer. Unlike the use of surrogates and direct measurements as a method of 
exposure assessment for background fields, estimation of exposure from appliances is poorly developed, generally consisting of the collection of data on frequency and duration of use via questionnaires (Kheifets \& Oksuzyan, 2008).

\subsubsection{Appliance Exposure}

The use of electric blankets has been investigated in various scenarios, exploring the association attached to childhood leukaemia in direct correlation to the child's use, while also the mother's pre- and post-natal use. The majority of studies have obtained similar findings whereby there is no association between both the child's use and the mother's pre-/post-natal use of electric blankets and the onset of childhood leukaemia (Savitz et al., 1990; London et al., 1991; Dockerty et al., 1998). Conversely, some also showed that the risk of childhood ALL was significantly increased when mothers had indicated electric blanket use during pregnancy $(\mathrm{OR}=1.59, \mathrm{Cl}: 1.11-2.29)$ (Hatch et al., 1998). However the lack of dose-response led the authors to conclude that the association found may not reflect MF exposure from electric blankets but with that of external exposure (Hatch et al., 1998).

\subsection{Brain Cancer}

As well as childhood leukaemia, in their original paper Wertheimer and Leeper (1979) also reported that children living close to high-voltage power lines presented increased risk of brain cancer. Brain cancer accounts for around $90 \%$ of all nervous system cancers and similar to leukaemia, onset is moderately rare with an annual incidence rate of 6 cases per 100,000 people in the USA (reviewed in Kheifets, (2001)). Brain cancer also correlates to leukaemia in that the causes for onset are likewise poorly understood and known factors, such as certain inherited genetic conditions and ionising radiation, only relate to a small proportion of cases. However, in the wake of additional studies indicating an increased risk of brain cancer in connection to ELF-MF exposure (Tomenius, 1986; Savitz et al., 1988), and in particular two types of malignant gliomas, glioblastoma and astrocytoma, succeeding studies have investigated them further. It originally appeared that the link to childhood brain tumour was more prominent than that of leukaemia (Ahlbom, 1988). Indeed, a more recent study has again published a positive association between high-level exposure $(>0.4 \mu \mathrm{T})$ and the risk of brain tumours in Japanese children under 15 years of age (Saito et al., 2010). 
However, the presence of only three cases and one control within this exposure group led to poor statistical precision with hugely variable confidence intervals presented $(\mathrm{Cl}$ : 1.05-113). In addition, a meta-analysis of childhood brain tumour studies has also indicated a non-statistically significant link when presenting risk estimates with multiple exposure assessment methods (Wartenberg, 1998). The majority of casecontrol studies however, have failed to provide evidence of a positive association when assessing exposure with a range of methods (Feychting \& Ahlbom, 1993; Gurney et al., 1996; Tynes \& Haldorsen, 1997; UK Childhood Cancer Study Investigators, 1999), meta-analysis (Mezei et al., 2008) or in conjunction with appliance use either directly by the child or the mother during gestation (Savitz et al., 1990; Preston-Martin et al., 1996). Overall, it can be concluded that there is little to no evidence which currently supports a link between ELF-MF exposure and the development of childhood brain cancer (Kheifets, 2001).

\subsection{Adult Studies}

The findings of Wertheimer and Leeper (1979) did not solely relate to childhood diseases as they also studied adults: a follow-up study in 1982 also found adult cancer to be associated with high-current wire configurations that were in close proximity to residences (Wertheimer \& Leeper, 1982). In light of these findings, a large number of epidemiological studies were performed to evaluate potential correlation between ELF-MF exposure and cancer. However, the majority of concerns are related to occupational exposure since chronic exposure to levels of ELF-MFs in occupational environments is extensively higher than those in a residential setting. Such occupational studies have used various designs, including population-based casecontrol studies, occupational cohorts, and death certificate surveys alongside several different exposure assessments, ultimately as a means of measuring either morbidity or mortality. The results of such studies are similarly conflicting, with some studies presenting a potential association between occupations considered to consist of high ELF-MF exposure and both leukaemia and brain cancer. The first occupational study outlined significantly increased mortality rates for total leukaemia occurrences (proportionate mortality ratios, $\mathrm{PMR}=1.4, \mathrm{Cl}$ : 1.1-1.6), in-particular acute leukaemia along with non-Hodgkin's lymphomas and malignant brain tumours among male 
electrical workers (Milham, 1985). The use of job titles taken from a death certificate database was used as a crude method of assessing exposure to magnetic fields. Employment in an 'electrical' profession such as an electrical and electronic technician, radio and telegraph operator, electrician, lineman (power and telephone), television and radio repairman, power station operator, welder and flame-cutter, and motion picture projectionist inferred to the researchers a higher than normal exposure to magnetic fields. A significant increase in brain tumour risk ( $\mathrm{OR}=2.2, \mathrm{Cl}: 1.1-4.1)$ also correlated with increasing probability of exposure to magnetic fields in a further casecontrol study which used occupation to classify exposure to magnetic fields (Lin et al., 1985). In an attempt to resolve the deficiencies in exposure assessment, industry workers wore personal dosimeters and exposures were catalogued. Questionnaires were combined with workplace measurements to establish a job exposure matrix (JEM) (Floderus et al., 1993), which in turn was used to further analyse the link between ELF-MF exposure at work and the risk of leukaemia and brain tumour in central Sweden. Jobs with exposures $\geq 0.29 \mu \mathrm{T}$ reported a strong association with all sub-types of leukaemia (OR = 1.6, $\mathrm{Cl}: 1.1-2.4)$, especially chronic lymphocytic leukaemia, yet not with any other sub-type of leukaemia or with brain cancer (Floderus et al., 1993). However, a further study on cancer mortality, in which the JEM was applied, found no association between an increased risk of either leukaemia or brain cancer mortality amongst electric utility company workers (Sahl et al., 1993). Similarly, a mortality study by Savitz and Loomis (1995) found no association with leukaemia mortality amongst a cohort of electric utility workers, except for those that had worked for more than twenty years as an electrician, in which case there was a 2.5fold increase in the risk of leukaemia. It is therefore unsurprising that a pooled analysis study including these studies presented no consistent exposure-response relationship or association with leukaemia or brain tumours (Kheifets et al., 1999). 
Table 5. The ELF-MF time-weighted average exposures for electrical occupations

\begin{tabular}{|l|l|}
\hline Occupation & Time-weighted average exposure $(\boldsymbol{\mu} \mathbf{T})$ \\
\hline Lineman (power \& telephone) & 3.6 \\
\hline Electrician / electrical engineer & 1.6 \\
\hline Power station operators & 1.4 \\
\hline Welders and flame-cutters & 2.0 \\
\hline Motion picture projectionists & 0.8 \\
\hline
\end{tabular}

A list of the time-weighted average exposures to ELF-MF for five selected jobs classified as electrical occupations. Adapted from IARC Working Group on the Evaluation of Carcinogenic Risks to Humans, (2002). Originally cited in Portier \& Wolf, (1998).

\subsection{Breast Cancer}

Similarly, the long-term exposure to ELF-MF has been hypothesised to increase the risk of breast cancer (Stevens, 1987). The idea was based on experimental evidence which suggested that ELF-MF affects the pineal glands production of the hormone melatonin in the same way that light does at night. Melatonin production follows a specific circadian pattern: low levels in daylight and high levels in darkness, and it is believed that high levels of melatonin could provide a protective effect against the development of breast cancer. Thus it is thought 50 and $60 \mathrm{~Hz}$ fields suppress melatonin production ultimately affecting oestrogen production by the ovary. Since then, and over the subsequent three decades, a considerable amount of epidemiological studies have analysed a potential association with breast cancer from ELF exposure in residential and occupational environments, including from electric blankets. However, most epidemiological studies have indicated little or no overall effect of ELF-MF exposure risks and similar findings are reported in the majority of studies of bed heating devices (Verkasalo et al., 1996; Li et al., 1997; Coogan \& Aschengrau, 1998; Feychting et al., 1998; Schoenfeld et al., 2003; Elliott et al., 2013). Studies have been established to explore both pre-menopausal (Vena et al., 1994) and post-menopausal breast cancer in conjunction with electric blanket use (Vena et al., 1991; McElroy et al., 2001). However, currently no association to breast cancer risk has been reported in either pre- or post-menopausal women using electric blankets (Gammon et al., 1998; Laden et al., 2000; Zheng et al., 2000b; Kabat et al., 2003). An exception is from a Norwegian case-control study (Kliukiene et al., 2004), where exposures were calculated based on nearby power lines. Effects were visible at quite low exposure levels, as any exposure to magnetic fields $\geq 0.05 \mu \mathrm{T}$ was associated with 
an increased risk of breast cancer. Similarly, the general outcomes from studies of occupational exposure did not indicate any effects either (Coogan \& Aschengrau, 1998; Kliukiene et al., 2004; Forssén et al., 2005; McElroy, 2007). Furthermore, the metaanalysis studies (Erren, 2001; Chen et al., 2010) which, between them, summarised the literature available from 1966-2009, including studies analysing residential and occupational exposures, as well as those from electric blankets. In all cases, both studies were in agreement that there was very little association between ELF-MF exposure and a proneness to female breast cancer.

Overall the evidence available is consistently negative and does not suggest an increased risk of breast cancer related to ELF-MF exposure. Indeed the evidence is sufficient enough for Feychting (2013) to conclude with confidence that ELF-MFs do not cause breast cancer and that focus should instead switch to developing more promising hypotheses on biophysical mechanisms. Indeed if there truly is a link between residential and occupational exposure to ELF-MF and cancer (excluding leukaemia) the constant presence of inadequate sample sizes results in inconsistent data and lack of a universally accepted conclusion.

\subsection{Cardiovascular Disease}

Reports have also surfaced in the 1960's linking ELF-MF exposure to cardiovascular irregularities and more recent studies further link exposure with a reversible relationship to reduced heart rate variability (HRV) (Sastre et al., 1998). Although a pooled study conducted within the same institute later failed to replicate such findings (Graham et al., 2000), a hypothesis was established that ELF-MF could affect heart rate variability (Savitz et al., 1999). It was theorised following evidence of an increased mortality from acute myocardial infarction and arrhythmia-related cardiovascular disease, but not chronic coronary heart disease, which was observed in workers employed for longer durations in occupations with elevated ELF magnetic field exposures (Savitz et al., 1999). Subsequent studies, including one directly replicating the original study (Sahl et al., 2002), and others specifically designed to replicate these findings through analysis of various specified endpoints, mostly failed to find any association of increased myocardial infarction morbidity (Ahlbom et al., 2004), arrhythmia (Johansen et al., 2002) or total cardiovascular disease mortality (Mezei et 
al., 2005; Cooper et al., 2009; Koeman et al., 2013). A solitary study however did evoke some support for the original hypothesis (Savitz et al., 1999), observing a non-

significantly increased risk for acute myocardial infarction (Håkansson et al., 2003).

However, the only two studies to illustrate an association between occupational exposure and ELF-MF relied on mortality records as a method of exposure assessment. Thus, when taken as a whole, the evidence does not support the notion of ELF-MF as a causative factor in the development of cardiovascular disease (Kheifets et al., 2007).

\subsection{Neurodegenerative Disease}

Due to an aging population neurodegenerative diseases are becoming more frequent, thus representing an extensive burden on public health. Since familial cases only account for a small percent of the vast majority of these diseases' aetiology, it can be safely assumed that lifestyle coupled with environmental factors play an essential role in their onset. Although not well developed, the potential effects of ELF-MF have been extensively researched in numerous epidemiological studies as a potential risk related to neurodegenerative diseases. Research has predominantly investigated the link to Alzheimer's disease (AD) and motor neuron disease, particularly amyotrophic lateral sclerosis (ALS). The risk of Parkinson's disease (PD) and dementia in general have also been analysed but to a lesser extent.

\subsubsection{Alzheimer's Disease}

The risk of Alzheimer's disease was initially outlined to be elevated 3-fold in association with primary occupations of low intensity ELF-MF exposure (Sobel et al., 1995), findings which have later been partially supported by Feychting et al. (1998). Subsequent cohort studies have then provided evidence that long-term occupational exposure to higher ELF-MF intensities (>0.2 $\mu \mathrm{T}$ ) also increased risk of AD 2.3-fold (Qiu et al., 2004), alongside that of AD mortality (Savitz et al., 1998; Feychting et al., 2003). Furthermore, age-specific analyses also indicated a stronger association with the onset of $A D$, with a proclivity for early onset $A D$ mortality (<75) (Feychting et al., 2003).

Conversely and in contrast to the data presented by the aforementioned studies, there has also been evidence presented which does not support the proposition that aboveaverage levels of ELF-MF are related to the onset of AD (Johansen, 2000; Noonan et al., 2002). Similarly, Sorahan and Mohammed (2014) also failed to establish a statistically 
significant trend associated with risk of AD mortality and increased ELF-MF exposure either over recent or primary occupations, or over a lifetime occupation in a cohort of UK electricity supply workers.

Again, inconsistencies on the basis of study design, the examination of morbidity or mortality coupled to the standard of exposure assessment has resulted in discrepancies between the data published. Ultimately, the data in which the risk of Alzheimer's disease is increased due to ELF-MF exposure are weak.

\subsubsection{Amyotrophic Lateral Sclerosis}

Another disease that has been extensively focused upon is motor neuron disease, the most common of which is amyotrophic lateral sclerosis (ALS), a fatal disease which permanently damages the nerve cells in the brain and spinal cord. A hypothesis whereby high occupational ELF-MF exposure increased the risk of ALS was further accentuated by a small case-control study which reported a potential 2.5 -fold increase in the risk of ALS in relation to occupational exposure to ELF-MF for more than 20 years (Davanipour et al., 1997). However the authors cautioned that owing to their method of exposure assessment and the small numbers of cases and controls used, their findings required confirmation in larger studies that use quantitative exposure data. Further support for an association was later offered from several well-designed cohort studies (Savitz et al., 1998; Johansen, 2000). Conversely, the results from studies which used the job exposure matrices (JEMs) to estimate MF exposure have been less consistent. Two studies reported ALS was not associated with ELF-MF exposure at any level (Noonan et al., 2002; Feychting et al., 2003), but both studies did in fact report significant associations of ALS attached with a history of workers in electrical professions, $(\mathrm{OR}=2.30,95 \% \mathrm{Cl}: 1.29-4.09)$ and $(\mathrm{OR}=1.4,95 \% \mathrm{Cl}: 1.1-1.9)$ respectively. Vergara and co-authors (2015) also applied JEM to analyse the relationship between occupational exposure to MFs and ALS mortality. Similarly, they did not find increased ALS mortality odds within high MF exposure categories when compared to low exposure but, consistent with previous publications, an association between electrical professions and ALS was observed. More recently, Sorahan and Mohammed (2014) investigated the risks of ALS mortality in a cohort of UK electricity supply workers and, unlike any of the aforementioned studies, failed to establish a 
statistically significant trend associated with risk of ALS and increased ELF-MF exposure either over a lifetime, recent or primary occupations. Additionally, two meta-analysis studies have analysed the previously reported associations of occupational magnetic field exposure with amyotrophic lateral sclerosis. Zhou et al. (2012) reported significant increases in risk of ALS due to ELF-MF exposure based on 9 case-control studies $(R R=1.39)$ but not in 8 cohort studies $(R R=1.16, C l: 0.80-1.69)$. Similar findings are illustrated in the meta-analysis from Vergara et al. (2013), who analysed 12 case-control studies reporting a significant increased risk of 1.38 for ALS, but again a weak associated risk of 1.14 from 9 cohort studies. The fact that ALS associations are stronger in studies which use occupational titles as a means of exposure measurement, rather than MF levels, suggests that magnetic fields are in fact not the explanation for the observed association of job titles with ALS.

\subsubsection{Parkinson's Disease}

The risk of Parkinson's disease has been analysed to a lesser extent than AD and ALS because epidemiological studies using either utility cohort studies, (Savitz et al., 1998; Johansen, 2000; Feychting et al., 2003; Roosli et al., 2007; Sorahan \& Mohammed, 2014) or a mortality registry study (Davanipour et al., 1997) have failed to provide any evidence of an association between elevated occupational ELF-MF exposure and PD. A meta-analysis study also failed to report any association between MF and PD (Vergara et al., 2013). Conversely, a positive association was observed in three separate methods of exposure assessment performed within one study (Noonan et al., 2002). However, owing to the inconsistent findings of this study, this positive association would need to be independently replicated in order to provide reliable support for an association between occupational ELF-MF exposure and Parkinson's disease.

In conclusion, there have been many studies into exposure to ELF-MF and risks of the collective group of neurodegenerative diseases, including several meta-analyses. Based on the literature, although largely contradictory and ultimately inconclusive, it is widely accepted that the extent of excess risk for Alzheimer's, Parkinson's and motor neurone disease assigned to occupational magnetic field exposure is probably small or, with regard to Parkinson's disease, most probably nil. Even in the case of $A D$, where studies ascribed a potential association to mortality, there remains a wide scope for 
potential publication bias and misclassification of the disease accounting for the observable risk (Johansen, 2000; Vergara et al., 2013).

Although epidemiological studies form the main body of evidence linking ELF-MF exposure to potential health effects, interpretation of data is problematic owing to inconsistent and unreliable methods of exposure assessment. In addition to this, any positive association related to an epidemiological study is based upon health risk estimates in human populations heavily biased towards cancer risk, while in vitro and in vivo studies represent a more reliable method of determining any potential genotoxic and carcinogenic effects of ELF-MFs through the use of defined exposure conditions and a variety of genotoxic endpoints. Yet, despite the numerous experimental studies performed using these improved parameters, their ability to evaluate whether power-frequency magnetic fields induce DNA damage has thus far failed to provide conclusive evidence for the mutagenic effects of extremely lowfrequency magnetic fields (AGNIR, 2001). It is however obstructive that the specific causes for the development of different types of human cancer are still poorly understood.

\subsection{Experimental Studies}

The development of cancer is a multistep process in which somatic cells acquire mutations in a specific clonal lineage (Loeb et al., 2003). The current understanding in the causation of cancer points to at least a two pathway system, of which neither path is mutually exclusive: a genotoxic pathway and an epigenetic pathway (Vijayalaxmi \& Obe, 2005). Yet in contrast to ionising radiation where the effects of exposure have been well established and the subsequent DNA damage has been extensively studied (reviewed in Lara et al., (2015)), there is currently no established mechanistic model for extremely low-frequency magnetic fields. The current epidemiological data however imply that extremely low-frequency magnetic fields may destabilise the genome of exposed cells, although it has been often outlined that ELF-MFs are themselves not genotoxic (Lacy-Hulbert et al., 1998; McCann et al., 1998). The process of a genotoxic pathway therefore assumes that ELF-MF exposure possesses the capability to induce DNA damage in a cell. Unrepaired DNA damage will result in DNA single- (SSBs) and double-strand breaks (DSBs), which are responsible for genomic 
instability. Moreover, the repair of DSBs is also an error-prone process, and incorrect repair of DSBs can result in the formation of chromosomal aberrations (CAs), micronuclei (MN), sister chromatid exchanges (SCE) and mutations that ultimately lead to carcinogenesis. Correspondingly, their unrepaired presence in germ cells has also been known to result in reproductive and birth defects. As a result, the possible defects to development and spermatogenesis in relation to ELF-MF exposure have also been confronted in various animal models. For the most part however, the results between studies are relatively consistent in illustrating a lack of adverse effects in regards to both development (reviewed in (Juutilainen, 2003; Juutilainen, 2005)) and spermatogenesis (Duan et al., 2014). Conversely, the experimental data produced in relation to the potential genotoxicity of ELF-MF exposure are reminiscent of that produced by epidemiological studies in that it is moderately conflicting. This point was typified by the intra-laboratory inconsistencies published by Luukkonen et al. (2011) and Luukkonen et al. (2014) who in contrast to their earlier study (Luukkonen et al., 2011), more recently illustrated that ELF-MF exposure (100 $\mu \mathrm{T}, 24$ hours) was able to induce micronuclei in human SH-SY5Y neuroblastoma cells at 8 and 15 days postexposure (Luukkonen et al., 2014).

Despite this, the majority of studies conducted to date have found no consistent evidence of magnetic field induced effects on genotoxicity using an array of field intensities: 0.001-1 mT (Paile et al., 1995; Luceri et al., 2005; Stronati et al., 2004; Testa et al., 2004; Luukkonen et al., 2011; Saha et al., 2014), and 5-400 mT (Antonopoulos et al., 1995; Tateno et al., 1998; Miyakoshi et al., 2000). Yet there have also been several studies, both in vivo and in vitro where positive correlations have been reported; for example a significant three-fold elevation in SCE was observed in mouse m5S cells exposed to $400 \mathrm{mT}(50 \mathrm{~Hz})$ for 40 hours (Yaguchi et al., 1999). Indeed one of the first attempts to analyse the direct genotoxic effects of ELF-MF in vivo was a study by Lai and Singh (1997). The authors reported that acute exposure (2 hours) of adult rats (Sprague-Dawley) to a $60 \mathrm{~Hz}$ MF at flux densities of $0.1,0.25$ and $0.5 \mathrm{mT}$, caused a dose-dependent increase in DNA single strand breaks in brain cells harvested at 2 and 4 hours post-exposure, while a dose-dependent increase in DSBs was also witnessed but only at 0.25 and $0.5 \mathrm{mT}$. However, several attempts have been made to 
independently validate these findings, using similar exposure conditions and experimental methods (alkaline comet assay performed on whole brain homogenates) but proved unsuccessful (McNamee et al., 2002, 2005). The sensitivity of such assays had previously been reported to equate to $10-50$ cGy of X-irradiation in lymphocytes (McNamee et al., 2000). Furthermore, earlier findings by Frazier et al. (1990) contradict those of Lai and Singh (1997) who reported the presence of strand breaks 4 hours after exposure to MF. Frazier and co-authors (1990) assessed the repair kinetics of DNA SSBs in the presence of $60 \mathrm{~Hz}$ magnetic field of $1 \mathrm{mT}$, noting that within 20 minutes of exposure between 50 to $75 \%$ of the induced SSBs had been repaired, while the majority of the remaining damage was repaired after 3 hours. However, a more recent study by Lai and Singh (2004) once again illustrated a significant increase in DNA damage in brain cells from whole brain homogenates taken from rats after 24- or 48 hours continuous exposure to $60 \mathrm{~Hz}, 10 \mu \mathrm{T}$ magnetic fields (Lai \& Singh, 2004). Similarly, a dose-dependent increase in DNA damage was reported in three cell lines (HL-60 leukaemia cells, Rat-1 fibroblasts and WI-38 diploid fibroblasts) exposed for 372 hours to $0.5-1.0 \mathrm{mT}, 50 \mathrm{~Hz}$ ELF-MF, while genotoxicity peaked at 24 and 72 hours (Wolf et al., 2005).

These discrepancies illustrate how the comparison of any corresponding data generated from experimental studies is difficult owing to differences between exposure conditions, designs and methods (Ruiz-Gomez \& Martinez-Morillo, 2009). Thus a collaborative European Union funded project known as the REFLEX project was established in an attempt to verify the data under controlled and standardised conditions and thereby eliminate any potential inter-laboratory inconsistencies. A series of studies from several laboratories ultimately led to a plethora of publications analysing the genotoxic effects of ELF-MF exposure (Ivancsits et al., 2002, 2003a, 2003b, 2005, Winker et al., 2005). The initial study was established to analyse the effects of continuous exposure to a $50 \mathrm{~Hz}$ sinusoidally magnetic field of $1 \mathrm{mT}$ for 24 hours on cultured human diploid fibroblasts (Ivancsits et al., 2002). Discrimination between DNA single- (SSB) and double-strand breaks (DSB) was achieved through the use of two separate assays: the alkaline comet assay detected both SSB and DSB, while the neutral comet assay was used to solely detect DSBs. No significant difference in the 
amount of DNA breaks were detected between continuously- and sham-exposed cells using either the alkaline or neutral comet assay (Ivancsits et al., 2002). However, previous data published by Nordenson et al. (1994) indicated a significant 3-fold increase of chromosomal aberrations in human amniotic cells exposed to a $50 \mathrm{~Hz}, 30$ $\mu T$ intermittent field for 72 hours (20 seconds on/20 seconds off). These findings are perhaps unsurprising given that continuous ELF-MF exposure as part of our daily environment is moderately sparse, since different electrical household appliances produce a range of exposure levels over short periods. Similarly, these findings may also suggest that biological and possibly adverse health effects are potentially related to exposure to intermittent magnetic fields (Kheifets et al., 1997). However subsequent attempts to replicate the findings observed by Nordenson and co-authors (1994) have thus far proven unsuccessful, with Galt et al. (1995) illustrating decreased chromosomal aberrations in similarly exposed amniotic cells. Nevertheless, Ivancsits and co-authors (2002) subsequently concentrated on optimising intermittent exposure conditions for maximal effect on DNA strand break levels. As such, intermittent on/off times were increased in 5 minute increments while the other experimental parameters remained constant $(50 \mathrm{~Hz}, 1 \mathrm{mT}$ for 24 hours). The highest level of DNA strand breaks in both the alkaline and neutral comet assays was induced using intermittent exposures of 5 minutes on and 10 minutes off. Furthermore, by using the optimal intermittent exposure conditions and varying the magnetic flux density between 0.02 $2 \mathrm{mT}$, significant increases were observed in both the alkaline and neutral comet assay at flux densities as low as $70 \mu \mathrm{T}$ (Ivancsits et al., 2002). Ensuing studies using identical exposure conditions ( $50 \mathrm{~Hz}$ sinusoidal, $1 \mathrm{mT}$, intermittent $5 \mathrm{~min}$ on/10 $\mathrm{min}$ off) for a period of 1 to 24 hours also reported an increase in alkaline and neutral comet tail factors in a time-dependent manner, with the largest being at 15 hours (Ivancsits et al., 2003b). The assay levels declined thereafter, without returning to basal levels. Moreover, increasing the magnetic flux density between 0.02-1 mT (15 hours, intermittent $5 \mathrm{~min}$ on/10 $\mathrm{min}$ off) resulted in a significant increase in DNA SSBs and DSBs at $35 \mu \mathrm{T}$ (Ivancsits et al., 2003b). Finally following this series of experiments the authors were able to define the optimum conditions at which the maximum effect on SSB- and DSB was evoked: $100 \mu \mathrm{T}$, intermittent (5 min on/10 min off), for 15 hours with human fibroblast cells from donors over 40 years of age, produced a 2-fold 
increase in DSB and a 4-fold increase in DSB plus SSB (Ivancsits et al., 2003a, 2003b). Although these effects were predominantly seen in human fibroblast cells, similar effects were also observed in human melanocytes and rat granulosa cells; however no detectable damage was witnessed in human lymphocytes, human monocytes or human skeletal muscles cells (Ivancsits et al., 2005). Comparable results were observed by Luceri et al. (2005) who failed to observe an increase in DNA damage in human blood lymphocytes exposed to $50 \mathrm{~Hz}$ magnetic fields at 1,10 or $100 \mu \mathrm{T}$ for 18 hours and more recently by Focke et al. (2010), who designed a study in an attempt to validate similar previously conducted studies (Ivancsits et al., 2002, 2003a, 2003b, 2005). Focke and co-authors (2010) were able to replicate an ELF-MF exposure dependent increase in comet tail DNA using three cell models of primary human fibroblasts (ES-1, HR-1d and MRC-5) intermittently exposed to $50 \mathrm{~Hz}$ ELF-MF at a flux density of $1 \mathrm{mT}$. However, statistical significance was smaller and with higher experimental variation than those previously presented by Ivancsits and co-authors (2002), while HeLa cancer cells were also not significantly affected (Focke et al., 2010). It could therefore be hypothesised that any observed inter-laboratory inconsistencies could be due to alternate responses to field exposure between cell types. However Kim et al. (2010) illustrated that DSBs were induced not only in normal human fibroblasts, but also HeLa cervical cancer cells, by both a continuous exposure of $6 \mathrm{mT}$ for 30 minutes and an intermittent exposure of $6 \mathrm{mT}$ for 30 minutes every 24 hours over 3 days. Further studies were performed within the REFLEX programme which analysed different genotoxic endpoints. ELF-MF exposure of intermittent fields (5 min on/10 min off) at a flux density of $1 \mathrm{mT}$ for 10 hours resulted in a significant 3-fold increase in micronuclei and a 10-fold increase in chromosomal aberration frequency above sham-exposure levels (Winker et al., 2005). Several subsequent studies have attempted to replicate these positive findings in a bid to finally obtain a general consensus of consistent data, however Scarfi et al. (2005) failed to confirm the REFLEX programme's findings using the alkaline comet and micronucleus assays, despite using identical fibroblast line and exposure conditions.

The reproducibility of data however must be achieved through the use of the most sensitive assay available at the time. The use of the comet assay for measurement of 
DSB is relatively insensitive and also unable to produce exact measurements of DSB yields (Burdak-Rothkamm et al., 2009). Indeed the use of the $\mathrm{yH} 2 \mathrm{AX}$ assay in which the sites of DSB are highlighted through the use of a fluorescently-tagged monoclonal antibody represents an advancement in the detection of DSBs over the conventional comet assays (reviewed in Kuo \& Yang, (2008)). The formation of $\mathrm{Y}-\mathrm{H} 2 \mathrm{AX}$ foci which represents the phosphorylated form of histone $\mathrm{H} 2 \mathrm{AX}$ is specific to DNA double-strand breaks. Indeed the phosphorylation of $\mathrm{H} 2 \mathrm{AX}$ is one of the primary characteristics of DSB in eukaryotes (Rogakou et al., 1998). Therefore Burdak-Rothkamm and co-authors (2009) re-assessed the genotoxic potential of ELF-MF by using the $\mathrm{\gamma H} 2 \mathrm{AX}$ assay as well as comparable techniques and parameters: $\mathrm{VH} 25$ fibroblast cells exposed to intermittent $50 \mathrm{~Hz}$ ELF-MFs at numerous flux densities (50, 100, 500 and $1000 \mu \mathrm{T})$ for 15 hours ( $5 \mathrm{~min}$ on/10 min off), alongside a commercial MF exposure system almost identical to that used in the REFLEX programme studies. A 15 hour continuous field was simultaneously analysed for each of the defined magnetic flux densities while a positive control (X-rays) dose-response curve was also defined for both the alkaline comet and $\mathrm{\gamma H} 2 \mathrm{AX}$ assays. Yet, despite the fact that the $\mathrm{\gamma H} 2 \mathrm{AX}$ method possessed the ability to reliably detect DNA damage equivalent to an X-ray dose of $0.025 \mathrm{~Gy}$, neither the alkaline comet assay nor the gamma $\mathrm{\gamma H} 2 \mathrm{AX}$ assay could detect significant damage at the DNA breakage level in the MF exposed fibroblasts and thus confirm the REFLEX programme's data. Similarly, neither the chromosome aberration nor the micronucleus assay detected any chromosomal damage in the MF exposed fibroblast cells (BurdakRothkamm et al., 2009). These findings are in conjunction with those of Scarfi and coauthors (2005) and more recently still Saha and co-authors (2014). Whilst using similar experimental parameters ( $50 \mathrm{~Hz}$ intermittent, $(5 \mathrm{~min}$ on/10 min off) magnetic field at $300 \mu \mathrm{T}$ for 15 hours), the findings of Saha et al. (2014) provide further evidence of an inability of ELF-MFs to cause direct DNA damage. Owing to its rapid recruitment to sites following the introduction of DNA DSBs, Saha and co-authors quantified the tumour suppressor p53 binding protein 1 (53BP1) foci numbers as a direct signalling response to the formation of DSBs. The quantification of 53BP1 foci numbers as a marker for DNA DSBs provided increased assay detection sensitivity. This in turn enabled the authors to detect a statistically significant increase in 53BP1 foci following a $10 \mathrm{mGy}$ dose $\mathrm{X}$-rays. Though, the minimum detectable response was calculated to 
equate to approximately 0.233 DSB per cell, which corresponds to an estimated dose of 22 mGy (Saha et al., 2014).

Whilst the establishment of the collaborative REFLEX programme further added to the inconsistent evidence regarding ELF-MFs genotoxic potential, the use of meta-analysis studies provides a method capable of counteracting inter-laboratory inconsistencies in data (Vijayalaxmi \& Obe, 2005; Ruiz-Gomez \& Martinez-Morillo, 2009). Indeed Vijayalaxmi and Obe (2005) utilised such an analysis, pooling together data from 63 in vitro studies conducted over a period of 13 years using human and animal somatic cells to analyse the overall effects of ELF-MF exposure, using single and/or double strand breaks, chromosomal aberrations, micronuclei and sister chromatid exchange as end points to define genetic damage. They found $22 \%$ of studies presented an increase in genetic damage in ELF-MF exposed cells. Comparatively, $46 \%$ of studies demonstrated no increase in damage while the remaining $32 \%$ of studies were inconclusive in their findings. More recently, similar results were observed when analysing solely DNA strand breaks induced by magnetic fields: $37.5 \%$ of studies found induction of DNA strand breaks while $62.5 \%$ of studies reported no effects (Ruiz-Gomez \& MartinezMorillo, 2009).

While ELF-MF could potentially be carcinogenic, the culmination of these findings appears to support the inability of ELF-MF exposure to cause a direct carcinogenic effect and fundamentally any potential role in the initiation of carcinogenesis can be confidently excluded. However, as was previously mentioned, the current understanding in the causation of cancer points to at least a two pathway system, the first of which being a genotoxic pathway while the second assumes ELF-MF partakes in an epigenetic role (Vijayalaxmi \& Obe, 2005).

\subsection{Combined ELF and Carcinogen Exposure}

An epigenetic role assumes that isolated ELF-MFs may not be genotoxic, but rather that the effects of ELF-MF exposure could either be enhanced or rather potentiate the effects of environmental carcinogenic agents such as chemicals, ionising radiation or ultraviolet (UV) radiation. Since humans are exposed to various combinations of an array of different carcinogens at changeable levels, exposure to ELF-MF could potentially act in a promotor, co-promotor or enhancer through either simultaneous or 
sequential exposure, ultimately contributing towards tumour progression (Juutilainen et al., 2000). Indeed a study had previously reported that mice experienced enhanced skin tumorigenesis when exposed to UV radiation in conjunction with magnetic fields (Kumlin et al., 1998). Additionally, evidence was presented that $50 / 60 \mathrm{~Hz}$ magnetic field exposure at flux densities of 50 and $400 \mathrm{mT}$ potentiated a small but significant increase in the X-ray induced DNA strand breaks in human glioma cells (Miyakoshi et al., 1999, 2000). Similar statistically significant enhanced chromatid-type aberrations were observed in mouse m5S cells pre-exposed to mitomycin C or $3 \mathrm{~Gy} X$-rays followed by $60 \mathrm{~Hz}, 50 \mathrm{mT}$ or $50 \mathrm{~Hz}, 400 \mathrm{mT}$ for 40 hours (Yaguchi et al., 2000). Moreover, multiple studies signified that cells pre-treated with the tumour initiator benzo(a)pyrene (BP), and then co-exposed with benzene and 0.8-1 mT ELF-MF introduced an increase in the comet tail moment (Moretti et al., 2005), as well as a significant dose dependent increase in the frequency of sister chromatid exchanges and micronuclei formation (Cho \& Chung, 2003). A combination of 3 mT ELF-MF and xenobiotic (N-methyl-NO-nitro-N-nitrosoguanidine (MNNG)) induced DNA damage in human leukocytes (Villarini et al., 2006), while exposure to ELF-MF at field strengths as low as $100 \mu$ T for 24 hours have been illustrated to increase the menadione-induced DNA damage and MN formation in human neuroblastoma cells (Luukkonen et al., 2011).

The majority of studies in which a positive correlation was discovered were as a result of ELF-MF exposure preceding the known carcinogen (Juutilainen et al., 2006). These results indicate that ELF-MF is more likely to function as an enhancer and amplify the initial DNA damage by the known carcinogen instead of acting as the initiator of the original mutagenic event (Cho \& Chung, 2003).

However the majority of these co-carcinogenicity studies are either inconclusive or negative in their findings (Juutilainen et al., 2000; Vijayalaxmi \& Obe, 2005), despite using numerous cytogenic assays (comet, MN and SCE) and combined treatments with multiple mutagens (mitomycin C or X-rays) at a range of high $50 \mathrm{~Hz}$ flux densities (80$1740 \mathrm{mT}$ ) (Maes et al., 2000). Similarly, a recent series of studies performed by the same laboratory demonstrated that 4 or 16 hour exposure to $60 \mathrm{~Hz}, 1 \mathrm{mT}$ ELF-MF in combination with ionising radiation ( $1 \mathrm{~Gy}$ X-rays) or $\mathrm{H}_{2} \mathrm{O}_{2}$ or cellular myelocytomatosis 
oncogene (c-Myc) activation did not have either an enhancing or synergistic effect on MN formation (Jin et al., 2012) or primary DNA damage (Jin et al., 2014). However, more recently the authors re-analysed the effects of ELF-MF exposure on DNA damage response in combination with the same mutagens through the formation of $\gamma-\mathrm{H} 2 \mathrm{AX}$ foci (Yoon et al., 2014). Similar to their earlier published data (Jin et al., 2012, 2014), 1 mT ELF-MF in combination with $1 \mathrm{~Gy}$ X-rays, or $\mathrm{H}_{2} \mathrm{O}_{2}$ or c-Myc activation did not potentiate the formation of $\mathrm{\gamma}-\mathrm{H} 2 \mathrm{AX}$ foci (Yoon et al., 2014). However, when the field strength was increased to $2 \mathrm{mT}$ over the same duration (6 hours), a combination of ELF-MF and ionising radiation was capable of inducing $\mathrm{Y}-\mathrm{H} 2 \mathrm{AX}$, yet both $\mathrm{H}_{2} \mathrm{O}_{2}$ and cMyc remained incapable of this. It should also be noted that a 6 hour exposure of a 2 mT ELF-MF administered alone induced an increase in $\mathrm{y}-\mathrm{H} 2 \mathrm{AX}$, which is in contrast to the earlier data reported by Burdak-Rothkamm and co-authors (2009).

The significance of previous findings has been portrayed as uncertain by Mairs et al. (2007) who claim that the determination of ELF-MFs carcinogenic potential to this date had been based chiefly on studies that, while adept at detecting widespread and extreme cellular damage, were unable to expose any potential subtle molecular alterations. In an attempt to detect any small-scale potential genetic damage the authors developed a more sensitive method of DNA-damage detection involving examination of microsatellite DNA sequences. The use of these non-coding DNA sequences in identifying radiation-induced mutations had previously been shown to represent at least a 1000 times more sensitivity than analysis of the hypoxanthineguanine phosphoribosyl transferase (HPRT) gene (Boyd et al., 2000). Through the analysis of three types of microsatellite sequence mutation (change in allele size, loss of heterozygosity and allelic imbalance), a $50 \mathrm{~Hz}$ magnetic field of $1 \mathrm{mT}$ in strength was shown to increase the mutagenic capacity of 0.3 and $3 \mathrm{~Gy} \mathrm{y}$-irradiation by factors of 2.62 and 2.75 respectively (Mairs et al., 2007).

Despite these findings it is essential that the validity of the positive epidemiological results be corroborated with long-term carcinogenicity studies in animals. As the onset of childhood leukaemia potentially represents the sole cancer-associated end-point to date, numerous animal studies have also focussed on the analysis of whether $50 / 60 \mathrm{~Hz}$ 
ELF-MF exposures can alter the incidence or onset of leukaemia and lymphomas in rodents (reviewed in Boorman et al., (2000)).

\subsection{Experimental Evidence for Carcinogenicity of ELF-MF}

These studies have several advantages over epidemiological studies in that exposure can be directly assessed while in epidemiological studies direct human measurements are often absent. However, such tests then need to be sensitive enough to extrapolate the data and predict accurately the carcinogenic risk to humans. This is generally accepted to be the case as $84 \%$ of carcinogenic agents in humans correlate in experimental animal studies. Indeed the correlation could have been higher but seven remaining agents had not been adequately tested (Wilbourn et al., 1986). Additionally, for those exposures which illustrated carcinogenicity in both humans and experimental animals, there is a common target organ in at least one animal study (Wilbourn et al., 1986). Moreover, in animal studies specifically analysing leukaemia and lymphoma, $100 \%$ of the chemicals that result in hematopoietic tumours in humans did so in rodents (Boorman et al., 2000). However, animal studies performed on a selection of rodent models have proven consistently negative for $50 / 60 \mathrm{~Hz}$ magnetic field exposures enhancing the risk of leukaemia. Multiple studies have also been conducted including chronic exposure bioassays with exposures for up to two and a half years with flux densities up to $5 \mathrm{mT}$ (Mandeville et al., 1997; Yasui et al., 1997; Boorman et al., 1999; McCormick et al., 1999), initiation and promotion studies (Shen et al., 1997), transgenic models (McCormick et al., 1998) and tumour growth studies (Sasser et al., 1996; Morris et al., 1999; Anderson et al., 2001).

Consequently, when taken together, these combined animal bioassays and the lack of detectable ELF-MF genotoxic damage in stimulated lymphocytes (Antonopoulos et al., 1995; Paile et al., 1995) weaken the epidemiologically hypothesised association between extremely low-frequency magnetic field exposures and leukaemia in humans (Wertheimer \& Leeper, 1979; Ahlbom et al., 2000). This is because any potential link to leukaemia would indicate stem or progenitor cells as a primary target cell for magnetic field exposure. 


\subsection{Classification}

In spite of the extensive research, there is no widely accepted consensus within the field as to the potential biological and carcinogenic effects of exposure to ELF-MFs. Yet, ultimately in 2002 the International Agency for Research on Cancer (IARC) published a monograph co-authored by an established working group composed of international experts, evaluating the carcinogenic risks to humans of non-ionising radiation. Categorisation of ELF-MF, as with other potentially hazardous biological agents, is evaluated by designated Working Groups in accordance with three separate criteria: a) carcinogenicity in humans, b) carcinogenicity in experimental animals, and c) mechanistic and other relevant data, relating to mechanisms of carcinogenesis. Such experimental evidence is then further evaluated in consideration of all relevant scientific data and classified by the strength of evidence that exposure to the specific biological agent is carcinogenic. This latter assessment relies on whether the evidence of exposure presents: i) sufficient evidence of carcinogenicity, ii) limited evidence of carcinogenicity, iii) inadequate evidence of carcinogenicity, or that iv) evidence suggests lack of carcinogenicity. Once each biological agent has been classified according to the strength of evidence relating to the three study models, the body of evidence is considered as a whole leading to an overall evaluation of the biological agent's carcinogenicity to humans. The five definitive groups that relate to an agent's carcinogenicity are described in Table 6. Of particular note is Group 2, where no quantitative measurement is attached to the terms 'probably' and 'possibly' carcinogenic. These terms are used by the Working Groups to signify the different quantities of published evidence indicating human carcinogenicity.

After reviewing all the data from studies up until this point (2002) the conclusion of the Working Groups was three-fold. First that bias, chance or confounding factors could not be confidently ruled out in any observation of a positive association between ELF-MF exposure and the occurrence of cancer, for which a causal relationship was considered. Secondly, that there was inadequate evidence in humans to establish a correlation for carcinogenicity in relation to all other cancers apart from childhood leukaemia; in other words that the available studies provided insufficient consistency, quality and/or statistical power to allow a conclusion regarding a causal association. 
Finally, they concluded that there was inadequate evidence in animal studies to characterise the carcinogenicity of ELF-MF since the studies could not be interpreted as showing either the presence or absence of a carcinogenic effect. Despite this, and due to the limited evidence from the epidemiological studies outlining a potential positive association with childhood leukaemia, the IARC working group classified extremely low-frequency magnetic fields within group $2 \mathrm{~B}$, outlining that exposure to ELF-MF is possibly carcinogenic to humans (IARC Working Group on the Evaluation of Carcinogenic Risks to Humans, 2002).

In retrospect, it has been suggested that a potential deciding factor of the classification passed down by the IARC was the persisting lack of an accepted biophysical mechanism through which extremely low-frequency magnetic fields can induce a genotoxic and/or carcinogenic effect. Yet, despite this inability to identify a plausible mechanism, a number of possibilities have been vigorously studied. 
Table 6. The IARC classification scale of carcinogenic risk to humans

\begin{tabular}{|c|c|c|c|}
\hline \multicolumn{2}{|c|}{ Group } & \multirow{2}{*}{$\begin{array}{l}\text { Explanation } \\
\text { There is sufficient evidence of } \\
\text { carcinogenicity in humans } \\
\text { OR } \\
\text { - Less than sufficient evidence of } \\
\text { carcinogenicity in humans, but, } \\
\text { sufficient evidence in experimental } \\
\text { animal studies and strong evidence of a } \\
\text { relevant mechanism of carcinogenicity. }\end{array}$} & \multirow{2}{*}{$\begin{array}{ll}\text { Examples } \\
\text { (Biological Agent) } \\
- & \text { Benzene } \\
- & \text { Etoposide } \\
- & \text { Engine exhaust } \\
& \text { (Diesel) } \\
- & \text { Formaldehyde } \\
- & \text { lonising radiation } \\
& \text { (all types) }\end{array}$} \\
\hline 1 & $\begin{array}{l}\text { Agent is } \\
\text { carcinogenic to } \\
\text { humans }\end{array}$ & & \\
\hline $2 A$ & $\begin{array}{l}\text { Agent is } \\
\text { probably } \\
\text { carcinogenic to } \\
\text { humans }\end{array}$ & $\begin{array}{l}\text { - Limited evidence of carcinogenicity in } \\
\text { humans and sufficient evidence in } \\
\text { experimental animals } \\
\text { OR } \\
\text { - Inadequate evidence of carcinogenicity } \\
\text { in humans and significant evidence in } \\
\text { experimental animal studies, coupled to } \\
\text { strong evidence that the carcinogenic } \\
\text { mechanism resonates in humans. }\end{array}$ & $\begin{array}{ll}\text { - } & \text { Acrylamide } \\
\text { - } & \text { Cisplatin }\end{array}$ \\
\hline 2B & $\begin{array}{l}\text { Agent is } \\
\text { possibly } \\
\text { carcinogenic to } \\
\text { humans }\end{array}$ & $\begin{array}{l}\text { - Limited evidence of carcinogenicity in } \\
\text { humans and less than sufficient } \\
\text { evidence in experimental animals. } \\
\text { OR } \\
\text { - Inadequate evidence in humans, less } \\
\text { than sufficient evidence in experimental } \\
\text { animals but supporting evidence from } \\
\text { mechanistic studies }\end{array}$ & $\begin{array}{ll}- & \text { Chloroform } \\
\text { - } & \text { Coffee (Urinary } \\
& \text { bladder) } \\
\text { - } & \text { Engine exhaust } \\
& \text { (Gasoline) } \\
\text { - } & \text { Extremely low- } \\
& \text { frequency } \\
& \text { magnetic fields } \\
\text { - } \quad \text { Lead }\end{array}$ \\
\hline 3 & $\begin{array}{l}\text { Agent is not } \\
\text { classifiable as to } \\
\text { carcinogenicity } \\
\text { to humans }\end{array}$ & $\begin{array}{l}\text { - Inadequate evidence in humans and } \\
\text { inadequate or limited in experimental } \\
\text { animal studies. } \\
\text { - Further research is needed }\end{array}$ & $\begin{array}{ll}- & \text { Chlorinated } \\
& \text { drinking-water } \\
- & \text { Diazepam } \\
- & \text { Extremely low- } \\
& \text { frequency electric } \\
& \text { fields } \\
\text { - } & \text { Fluorescent } \\
& \text { lighting } \\
\end{array}$ \\
\hline 4 & $\begin{array}{l}\text { The agent is } \\
\text { probably not } \\
\text { carcinogenic to } \\
\text { humans }\end{array}$ & $\begin{array}{l}\text { - Evidence suggests a lack of } \\
\text { carcinogenicity in humans and in } \\
\text { experimental animal studies. }\end{array}$ & - Caprolactam \\
\hline
\end{tabular}

The current IARC classification categories and the corresponding criteria used to evaluate the carcinogenic potential of biological agents in humans. Category criteria were taken from the IARC Working Group on the Evaluation of Carcinogenic Risks to Humans, (2013). The biological agent examples for each classification were taken from IARC Working Group on the Evaluation of Carcinogenic Risks to Humans, (2016), where a comprehensive list of agents and their current classification can also be located. 


\subsection{Mechanism}

\subsubsection{The Melatonin Hypothesis}

The first hypothesised mechanism is referred to throughout the literature as the 'melatonin hypothesis' (Stevens, 1987; Stevens \& Davis, 1996). Melatonin ( $N$-acetyl-5methoxytryptamine) is a mammalian neuro-hormone, which is synthesised and secreted predominantly by the pineal gland (Lerner et al., 1959). Although the gastrointestinal tract (GIT), lymphocytes and skin also contribute as alternate sources of melatonin (Bubenik, 2002; Carrillo-Vico et al., 2004; Slominski et al., 2008). The synthesis and secretion of melatonin is a biomarker of circadian rhythmicity which is controlled by a day-night cycle and more specifically by light and dark, in that darkness initiates and light terminates its production (Moore et al., 1967; Lewy et al., 1980). The presence of light is detected by non-rod and non-cone receptors in the retina. Once the light intensity declines below a threshold of 10 lux (Henshaw \& Reiter, 2005), a signal is sent via the retinohypothalmic tract to the suprachiasmatic nuclei (Okamura et al., 2002), which in turn prompts the regulation of melatonin production, primarily in the pineal gland through the parenchymatous cells (Singh \& Jadhav, 2014).

Melatonin plays a key regulatory role in many important physiological processes which occur with a daily or seasonal synchronisation, such as metabolism regulation, reproduction, sleep (reviewed in Singh \& Jadhav, (2014)) and the modulation of hormones (reviewed in (Pandi-Perumal el al., 2006; Slominski et al., 2012)). Moreover, a reduced melatonin concentration has been reported post-magnetic field exposure (Welker et al., 1983), while the suppression of melatonin has been linked to various diseases, including cancer (reviewed in Hill et al., (2011)). Therefore the theory originally devised by Stevens (1987) proposing an ELF-MF associated nocturnal reduction in melatonin synthesis represents a tentative explanation as to the potential oncogenic action of magnetic fields (Wertheimer \& Leeper, 1979).

Ultimately, since the inception of the 'melatonin hypothesis', the effects of ELF-MF exposure on melatonin secretion have been extensively studied. The majority of in vivo studies have provided very little evidence to suggest ELF-MF reduces melatonin synthesis. However, whilst covering a variety of exposure parameters, these have predominantly occurred in short-term studies ( $\leq 2$ weeks of exposure duration) 
conducted on rodents (Vanderstraeten et al., 2012). The issue with this is therefore two-fold as the majority of studies have been conducted on a nocturnally active species whose anatomical location of the pineal gland is largely different from that of humans (Touitou et al., 2006). Moreover, that long-term in vivo studies have failed to provide a definitive conclusion is perhaps best highlighted by a series of 6 -week studies conducted in male rats, which assessed the effects of ELF-MF exposure on pineal and plasma melatonin concentrations (Kato et al., 1993, 1994a, 1994b, 1994c, 1994d). The initial study displayed significantly reduced pineal and serum melatonin concentrations in male Wistar-King rats post-ELF exposure to a circularly polarised magnetic field up to $250 \mu \mathrm{T}$ (Kato et al., 1993), although normal production was restored one week following the termination of exposure (Kato et al., 1994d). Similar nocturnal suppression of melatonin levels were also seen in Long-Evan rats (Kato et al., 1994a). However, a subsequent study using the same exposure protocol on Wistar-King rats, but with horizontal or vertical polarised ELF-magnetic fields instead, had no effect on melatonin concentration levels (Kato et al., 1994c). However, the authors failed to present a clear explanation as to how these different polarised power-frequency magnetic fields could evoke such variations in pineal and plasma melatonin concentrations. Further studies investigating the long-term effects of magnetic fields on the pineal and plasma melatonin concentrations were equally inconsistent with no clear justification presented for as to why (IARC Working Group on the Evaluation of Carcinogenic Risks to Humans, 2002).

While some human volunteer studies have produced similarly contradictory results, it must be noted that the majority have reported a lack of an ELF-MF effect upon melatonin concentration following acute exposure of healthy volunteers (reviewed in Touitou et al., (2006)). On the other hand, the effect of chronic exposure in humans has been less comprehensively considered, although those that have been conducted seemingly confirm a lack of effect. This was illustrated in a study of workers exposed to both daily residential and occupational $50 \mathrm{~Hz}$ fields over a period of 1-20 years, whereby no change was detected in either the circadian synthesis of melatonin or their plasma melatonin concentration (Touitou et al., 2003). An issue with this study is that melatonin synthesis predominantly occurs during sleep therefore only nocturnal 
residential exposure principally applies. A pooled analysis of epidemiological risk estimates, based on either nocturnal or 24-hour residential exposure, concluded that there was no difference between the two (Schuz et al., 2007).

Ultimately, in light of the present literature, the melatonin hypothesis stating that disruption of melatonin secretion is a main factor in the carcinogenic effects of magnetic fields is not supported by either epidemiological or experimental data (Lewczuk et al., 2014), and thus should be regarded as negatively corroborated and rebutted (Vanderstraeten et al., 2012). Furthermore, the core of the original hypothesis was conceived on the potential association between ELF-MF and breast cancer (Stevens, 1987), which has also been dismissed (Feychting, 2013). It is also important to note that there is an equal absence of any valid mechanism by which changes in human melatonin concentrations could result in adverse effects on health or well-being.

\subsubsection{Radical Pair Mechanism}

Since it is widely accepted that ELF-MF does not itself possess sufficient energy to directly cause DNA breaks, several reports have suggested that such DNA and cytotoxic damage may result from an indirect secondary process. The most plausible hypothesis through which ELF-MF induced biological effects occur is the radical pair mechanism (RPM) (Timmel et al., 1998; Brocklehurst, 2002; Hore, 2012). Upon formation of a free radical, the unpaired electron within the outermost atomic orbital can be identified as existing in one of two spin states: $\uparrow$ or $\downarrow$ (Rodgers \& Hore, 2009). Free radicals, predominantly consisting of hydrogen peroxide $\left(\mathrm{H}_{2} \mathrm{O}_{2}\right)$, hydroxyl radicals $(\mathrm{OH})$ and superoxide radicals $(\mathrm{OOH})$, are created as a by-product of normal aerobic metabolism (Singh \& Jadhav, 2014). Collectively known as reactive oxygen species (ROS) they occur through the conversion of molecular oxygen into water and the subsequent release of electrons from the mitochondrial electron transport chain into the respiratory system (Singh \& Jadhav, 2014). Owing to the presence of the unpaired electron, free radicals may be highly reactive species either donating or accepting an electron. The formation of a radical pair therefore comprises two transient radicals created in tandem in which their unpaired electron spins are correlated in either an antiparallel (singlet state (S),

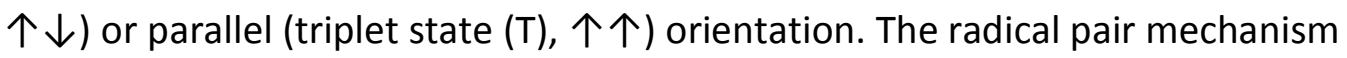


therefore hypothesises that magnetic field interaction alters the correlated spin dynamics of radical pairs, though the process through which this occurs is as yet unconfirmed (Hore, 2012).

In addition to the RPM, it has been proposed that a weak magnetic field enhances the lifespan of free radicals in cells, while it has been shown that the yield of free radical recombination can alter by 15 to $30 \%$ in the presence of a weak magnetic field (Eveson et al., 2000). Similarly, several studies have also shown increased formation of ROS after exposure to ELF-MF (Ciejka et al., 2011; Patruno et al., 2015). This most likely occurs through the Fenton reaction (Lai \& Singh, 2004), which is an iron-mediated catalytic process through which hydrogen peroxide is converted into more potent and toxic hydroxyl free radicals (Phillips et al., 2009) (Figure 3). These findings therefore correlate with recent data suggesting iron may accentuate the effects of ELF-magnetic fields (Lai \& Singh, 2004), whereby the addition of iron ions as a stimulator of the oxidation procedure alongside exposure to a $7 \mathrm{mT} 50 \mathrm{~Hz}$ magnetic field, significantly enhanced the number of damaged cells (Zmyslony et al., 2000). Additionally, an observed increase in DNA single strand breaks in rat brain cells following acute $60 \mathrm{~Hz}$ magnetic field exposure (Lai \& Singh, 1997) was ascribed to an increased presence of iron within brain cells (Singh \& Lai, 1998). Likewise both in vitro and in vivo studies have indicated enhanced lipid peroxidation and oxidative damage after ELF-MF exposure (Zmyslony et al., 2004; Yokus et al., 2005). However, similarly to genotoxic studies, others have noted that ELF-MF exposure fails to contribute to intracellular ROS levels (Hong et al., 2012) and ultimately, to the ability to produce significant amounts of oxidative damage in cells (Focke et al., 2010). 


$$
\mathrm{Fe}^{2+}+\mathrm{H}_{2} \mathrm{O}_{2}
$$

$$
\mathrm{Fe}^{3+}+\mathrm{OH}+\mathrm{OH}^{-}
$$

Figure 3. The Fenton reaction. Hydrogen peroxide in the presence of a transition metal, particularly $\mathrm{Fe}^{2+}$ produces the most potent reactive oxygen species, the hydroxyl radical.

A build-up of intracellular ROS is widely recognised as a causative factor in the oxidative damage of nuclear DNA, membrane lipids and cytosolic proteins. Moreover, the increased formation of reactive oxygen species is believed by many to be the primary oncostatic event in the formation of the majority of cancers (WHO, 2007). Thus enhanced levels, especially in the lymph nodes and bone marrow could subsequently lead to increased carcinogenesis, especially of lymphomas and leukaemia (Simko \& Mattsson, 2004). Several cellular defence mechanisms however exist in oxygen-metabolising cells to prevent free radical-induced oxidative damage (LimonPacheco \& Gonsebatt, 2009). The first of which includes the catalytic removal of free radical and ROS by radical scavengers, peroxidase and thiol-specific antioxidants and antioxidant-related enzymes such as catalase (CAT) and superoxide dismutase (SOD) (Simko \& Mattsson, 2004; Hong et al., 2012). Additionally, free radicals can be further reduced by electron donors such as glutathione (GSH) and vitamin $\mathrm{C}$, alongside the presence of proteins such as stress and heat shock proteins which protect against macromolecular damage and the regulation of redox-sensitive signalling pathways (Limon-Pacheco \& Gonsebatt, 2009). In fact, in addition to the melatonin hypothesis, melatonin is also a potent receptor, independent scavenger of free radicals and antioxidant, especially hydroxyl and peroxyl radicals (Allegra et al., 2003), while also playing a role in the stimulation of additional antioxidant synthesis (Reiter et al., 2003). As such, melatonin may play a fundamental role in the protection of the cytosolic proteins, membrane lipids and nuclear DNA against oxidative damage not only in adults but also in developing foetuses' (Garcia et al., 2014). For example, melatonin in animals has been illustrated by numerous studies to be highly protective of the foetus against oxidative damage (reviewed in Henshaw \& Reiter, (2005)). This suggestion is of note following the proposed general model pertaining to the onset and progression of childhood leukaemia which assumes that any initial event(s) occurs in utero (Greaves, 1999) (Chapter 1.2). While the same is potentially true in pregnant women where 
Nakamura et al. (2001) illustrated that serum melatonin possesses a diurnal rhythm which rises substantially following 24 weeks gestation and remains elevated until the full term. Indeed the addition of radical scavengers and antioxidants were able to inhibit an ELF-MF related increase in cell proliferation (Wolf et al., 2005), the MFinduced production of superoxide radical anions (Mannerling et al., 2010) and also prevent the previously observed induction of single- and double-strand breaks in brain cells of rats following ELF-MF exposure (Lai \& Singh, 1997). Under normal cellular conditions, ROS and antioxidants exist in equilibrium; however there have been studies which have outlined alteration in the free radical homeostasis in cells following ELF-MF exposure (Lupke et al., 2004; Simko \& Mattsson, 2004). An ELF-MF induced imbalance in intracellular ROS concentration, either through excessive production of ROS or deterioration in the cells' antioxidant defensive capabilities ultimately leads to cellular oxidative stress (Limon-Pacheco \& Gonsebatt, 2009). This has been suggested by the studies indicating ELF-MF exposure inhibits melatonin production and the CAT antioxidant defence (Akdag et al., 2010), while also enhancing ROS concentrations (Patruno et al., 2015). However, a study by Hong and co-authors (2012) analysed whether ELF-MF exposure of cultured human breast epithelial MCF10A cells could elicit oxidative stress by measuring intracellular ROS levels as well as antioxidant enzyme activity within these exposed cells. They found that exposure to $60 \mathrm{~Hz}$ ELF-MF at $1 \mathrm{mT}$ for four hours did not affect intracellular concentration of ROS in MCF10A cells (Hong et al., 2012). Likewise, SOD activity, which is recognised as the primary defence response to cellular oxidative stress, remained unaffected following exposure (Hong et al., 2012). Oxidative stress has been linked to numerous disorders but is most prominently recognised as the biological hallmark of neurodegeneration as well as the aging process (Hardeland, 2005; Limon-Pacheco \& Gonsebatt, 2009). Correspondingly, persistent or excessive cellular oxidative stress may ultimately induce oxidative damage to the macromolecules and lipid peroxidation which, if left unrepaired and subsequent genomic stability fails to be restored, could result in cell death via apoptosis (Kim et al., 2010). Apoptosis is a protective mechanism through which damaged, infected or potentially cancerous cells are removed to maintain normal homeostasis of multicellular organisms (Lacy-Hulbert et al., 1998; Juutilainen et al., 2000). In fact the alteration of the cells redox state to a more oxidative environment 
has been shown to occur immediately prior to the final phase of caspase activation in numerous models of apoptosis (reviewed in Kannan \& Jain, (2000)).

Yet, while the inhibition of apoptosis is another related candidate mechanism, the data correlating to the effects of ELF-MF on apoptosis remain rather limited and contradictory.

\subsubsection{Apoptosis}

As discussed above, a number of studies have consistently illustrated that apoptotic pathways were activated following continuous ELF-MF exposure (Simko et al., 1998; Akdag et al., 2013; Kim et al., 2014) at field intensities as low as $14 \mu \mathrm{T}$ (Kim et al., 2009). Similarly, the induction of apoptosis in both HeLa (human cervical carcinoma) cells and human lung fibroblast cells was illustrated in an additional study by Kim and co-authors (2010), following repetitive 30 minute exposure to an intermittent $60 \mathrm{~Hz}, 6$ mT magnetic field every 24-hours for 3 days (Kim et al., 2010). However, it must be noted that while Simko and co-authors (1998) reported an induction of apoptosis in human squamous cell carcinoma cells, continuous exposure to a range of $50 \mathrm{~Hz}$ field intensities (0.1-1 mT) for various time periods (24-, 48- or 72-hours) failed to indicate any statistically significant difference in apoptotic induction in human amniotic cells (Simko et al., 1998). Further contradicting data were presented, demonstrating that ELF-MF exposure had no statistically significant effect on apoptosis induction (Ding et al., 2004), either directly or 24 hours after acute (2-hour) exposure to a $1 \mathrm{mT}$ magnetic field (McNamee et al., 2002), or succeeding exposure to $50 \mathrm{~Hz}$ magnetic fields (100 or $300 \mu \mathrm{T}$ ), using an assay which possessed a detection limit of apoptotic frequency equivalent to that induced by exposure to 25 mGy X-rays (Saha et al., 2014).

Despite the widespread presence and functions of radicals within biological systems, the conflicting studies make it abundantly clear that further experimental and theoretical work is needed to fine-tune the conditions relating to ELF-MF sensitivity (Messiha et al., 2015). It follows that it is a fallacy to rationalise that biological systems are so fragile that any minor alteration in the homeostasis of intracellular radical concentration arising from interaction with weak magnetic fields could have a genotoxic effect (Hore, 2012). One exception would arise where exposure was in the presence of an amplification mechanism or the exposed system exhibited an already 
weakened defence system (Eveson et al., 2000). Nevertheless, the radical pair mechanism remains to date the most feasible method through which environmentally weak magnetic fields interact with our biochemical systems.

\subsection{Mutation Detection Methods}

As has been highlighted throughout this thesis, advancements in technological capabilities have subsequently led to a now ubiquitous exposure to a range of electromagnetic fields. It has therefore become a necessity to analyse and understand any potential biological effects ELF-MF may have on living organisms. However, over the years, a body of controversial and contradictory literature has been produced in regards, not only to the effects on the health of the general population, but also those caused at the DNA level. It has been suggested that the cause of these findings may reside in the methodologies employed throughout these studies (Mairs et al., 2007). It is reasoned that while the classical methodologies possess the ability to identify intense, widespread cellular DNA damage, they potentially lack the sensitivity required to reveal the more subtle molecular alterations that lead to possibly harmful genetic damage. Instead, an analysis of the rate at which mutations are induced in male mice following ELF-MF exposure could potentially rectify such assay sensitivity limitations.

For more than half a century the mouse model has played an authoritative role in the production of germline mutation induction data following exposure to chemical mutagens and ionizing radiation (Shelby, 1996; UNSCEAR, 2001). The use of pedigreebased assays, namely the specific-locus test (SLT) and the dominant lethal test (DLT), are responsible for providing the majority of experimental data. The germline mutation data generated in mice by these assays are of note since they can ultimately be extrapolated and used to assess the genetic risk of human exposure to such mutagens.

\subsubsection{Specific-Locus Test}

The development of the specific locus test (SLT) enables the accurate, non-subjective detection of viable mutations in the germ cells of mice (Searle, 1974). Developed in 1948 , the SLT employs the use of a genetic tester- $(T)$ stock mouse, which are homozygous recessive at seven specific loci (Table 7), six of which relate to the pigmentation of the mouse's coat: agouti, brown, chinchilla at albino, dilute, piebald- 
spotting and pink-eyed dilution, while the seventh controls ear morphology: short-ear (Russell, 1951; Russell, 1989). The predominant use of the specific-locus $T$-stock led to the test being commonly referred to as the Russell-7-loci test (Russell, 1951; reviewed in (Russell, 2004)), yet a second tester-stock mouse was constructed consisting of different loci known as the Harwell-test stock (Lyon \& Morris, 1966). Ultimately, through the use of seven easily recognisable visible markers, the SLT provides a platform through which the transmission of novel and heritable gene mutations can be scored. Indeed, the main strength of this assay is that it analyses the transferal of novel germline mutations in the offspring of treated parents, which simulates the genetic changes associated with heritable human genetic diseases (reviewed in Yauk et al., (2015)).

The assay functions as wild-type parental mice that have either been exposed to mutagens or left unexposed (control) are mated with $T$-stock mice. Owing to the homozygous recessive nature of the $T$-stock mice the majority of progeny will appear wild-type. However, a novel mutant allele (either dominant or recessive) which presumably occurred in the germline of the wild-type parent at any of the seven loci before fertilisation would manifest itself as a distinctive phenotypic change in the offspring (Favor, 1999). The scoring of both the exposed and control parental offspring provides an assessment of both the mutagen-induced and spontaneous mutation rates. Thereby, providing an assay through which mutagenesis can be calculated in the germline and potentially extrapolated to represent whole-genome rates (Davis \& Justice, 1998). Ultimately, this has led the SLT to become one of the most efficient methods of mutation induction analysis to date, through which the majority of knowledge pertaining to radiation and chemical mutagenesis has been provided (Selby, 1998). However, the SLT is not without its limitations. The main underlying issue with the SLT is that the average spontaneous mutation rates at the seven protein-coding genes are very low $\left(6.6 \times 10^{-6}\right)$ (Russell \& Russell, 1996). Since the induction of mutations is measured using a pedigree-based analysis, the SLT requires the use of hundreds to thousands of mice in order to accrue sufficient data through which any significant effect on mutation induction can be determined. Furthermore, the seven specific loci employed herein are each 600 to 5500 base pairs in length 
(Russell, 2004). As such the process of conducting this assay whilst additionally analysing de novo mutants, becomes tremendously expensive and time consuming. Consequently, laboratories around the world have discontinued the routine maintenance of the specific locus tester- $(T)$ stock mouse.

Table 7. The seven specific-locus test loci

\begin{tabular}{|l|l|l|l|}
\hline Name & Symbol & Protein & Chromosome \\
\hline Agouti & $a$ & $\begin{array}{l}\text { Melanocortin-receptor } \\
\text { antagonist }\end{array}$ & 2 \\
\hline Brown & $b$ & Tyrosine-related protein & 4 \\
\hline Albino & $c$ & Tyrosinase & 7 \\
\hline Pink-eyed dilution & $p$ & Tyrosinase-routing protein & 7 \\
\hline Dilute & $d$ & Myosin Va & 9 \\
\hline Short-ear & $s e$ & Bone morphogenetic protein & 9 \\
\hline Piebald spotting & $s$ & Endothelin receptor, type B & 14 \\
\hline
\end{tabular}

Details of the seven loci employed as markers in the specific-locus test (Russell-7 loci), (Modified from Russell, (2004)).

\subsubsection{Dominant Lethal Test}

The dominant lethal (DL) test is an extensively used test in the analysis of mutagenicity, measuring the genetic changes in germ cells by using embryonic mortality as an endpoint in rodents. The assay is predominantly conducted to evaluate the mutagenic consequences of paternal exposure, whereby males are either exposed to ionising radiation or used as controls and mated with an untreated female (Green et al., 1987). The manifestation of a dominant lethal mutation derived from a solitary parent could, in theory, occur at any period following fertilisation however, this assay is based solely upon the analysis of pre- and post-implantation death of the zygote. As such, females demonstrating signs of mating are sacrificed approximately seventeen days post-conception, upon which the ovaries and uterine contents are assessed. The amount of ovulated eggs is determined via counting corpora lutea, in addition to the number of viable and dead implanted embryos (reviewed in Yauk et al., (2015)) and the number of implants (living vs dead) against the number of eggs ovulated per pregnant female for both the treated and control groups. The dominant lethal effect is then calculated by comparing the data for the treated and control groups against one another. Indeed a true mutagenic effect, as expressed by the DL assay, would equate to a statistically significant increase in dead embryos per pregnant female or a 
statistically significant decrease in viable embryos per pregnant female when compared to a non-exposed control.

The DL method has been instrumental in identifying numerous mutagenic agents since its inception (Luning \& Searle, 1971; Searle \& Beechey, 1981; Kirk \& Lyon, 1984). Yet, since the DL assay is primarily employed to discover mutagens that cause chromosomal aberrations using embryonic death functions as the end-point, the assay fails to provide a definitive outcome, considering that additional genetic events and environmental factors may also heavily influence the occurrence of in utero loss of embryos. Moreover, similar to the SLT, the DL also implements a pedigree-based analysis, which again necessitates the use of a considerable amount of animals, whilst also being expensive and time consuming. Finally, owing to an elevated spontaneous mutation frequency attached to dominant lethal mutations, the DL assay sensitivity regarding the detection of small increases in mutation frequency is restricted (Russell \& Matter, 1980). Such limitation in sensitivity presented by both methods means that only exposure to relatively high doses of radiation can be analysed in sufficient detail. This proves problematic when attempting to replicate the effects of environmentally low and intermediate dose exposures. Furthermore, in light of progressive societal developments and an increased public and media awareness with regards to animal testing, the use of such numbers of mice is no longer accepted within modern society. Therefore more economical and sensitive experimental techniques were required in order to further progress the analysis of radiation-induced mutation induction.

\subsubsection{Hypervariable Tandem Repeat Regions}

The development of any assay would have to include a genetic system that evokes a high rate of spontaneous mutations in order to allow it to be sensitive enough to detect an alteration in DNA structure induced by a dose representative of that within the environment, whilst using a relatively small population sample. Following the successful utilisation of an individual's unique DNA fingerprint in both forensic science and the determination of family relationships (Jeffreys et al., 1985b; Jeffreys, 1987), alongside eukaryotic genome ubiquity, the use of hypervariable tandem repeat loci was implemented as a biomarker in the detection of mutation induction at the DNA level (Dubrova et al., 1993). The use of hypervariable tandem repeat regions not only 
provides all the characteristics required to work as an effective system in the analysis of the effects of mutagenic agents but also improves upon the two traditional approaches (SL \& DL tests). Firstly, such regions are known to be highly unstable, possessing spontaneous mutation rates in both germline and somatic cells thought to be at least a thousand times higher than in most protein-coding loci (Jeffreys et al., 1985a, 1991; Kelly et al., 1989). Moreover, the majority of repeat regions are primarily located in non-coding regions of most eukaryotic genomes, including human and mouse, so the identification of mutations ought not to be affected by the selection process (Jeffreys et al., 1985a; Kelly et al., 1989; Gibbs et al., 1993; Bois, 2003). Finally, any mutagenic response at these loci manifests as an alteration in repeat copy number, through either an insertion or deletion of repeat sequence, which translates as an easily detectable alteration in allele length. Thus, the detection of de novo mutant alleles occurs through quantifying changes in length relative to progenitor alleles (Jeffreys et al., 1988; Kelly et al., 1989). The resulting differentially sized alleles can easily be resolved through gel electrophoresis and Southern blot hybridisation. Additionally, the application of multi-locus DNA probes can provide simultaneous analysis of multiple loci which further boosts statistical power compared to the previous conventional approaches. Overall, the development and use of these loci has provided a highly informative laboratory-based biomarker in the analysis of the effects of mutagenic agents, allowing the detection of induced mutations at lower dose exposures within smaller population samples (Dubrova et al., 1993, 1998b, 2000a).

Tandem repeat sequences can be broadly divided into different classes according to their differing properties: total sequence size, length of repeat unit sequence and total number of repeats (Table 8). In fact, expanded simple tandem repeat (ESTR) loci were initially categorised as minisatellites, owing to the possession of some similar characteristics. However, subsequent characterisation of the loci has determined that ESTR regions are currently located solely within the mouse genome and are separate to the less hypervariable true minisatellite loci encompassed within the mouse genome (Kelly et al., 1989; Gibbs et al., 1993; Bois et al., 1998a, 1998b). As such, to date there is no animal model in which the mutation induction at minisatellites can be 
appropriately studied, nor is there any such equivalent for a human ESTR model at this time.

Table 8. Tandem repetitive sequences

\begin{tabular}{|l|l|l|l|}
\hline $\begin{array}{l}\text { Tandem Repetitive } \\
\text { Sequence }\end{array}$ & Sequence Size (nt) & Number of repeats & Total Size \\
\hline Microsatellites & $2-5$ & $10-100$ & - \\
\hline Minisatellites & $10-100$ & Up to 6000 & Up to $30 \mathrm{~kb}$ \\
\hline ESTRs & $4-9$ & Up to 4000 & Up to $20 \mathrm{~kb}$ \\
\hline
\end{tabular}

The structural differences tandem repetitive sequences. Data compiled from reviews: (Bois \& Jeffreys, 1999; Yauk, 2004; Yauk \& Polyzos, 2005; Bouffler et al., 2006; Somers, 2006).

\subsubsection{Minisatellites}

Minisatellites typically contain GC-rich repeat sequences that range from 10 base pairs to those over 100 base pairs in length (Table 8). Although extensively researched in humans (Jeffreys et al., 1985b, 1999; Jeffreys, 1987), DNA fingerprint analysis has shown that they are omnipresent throughout the genome of most organisms and higher eukaryotes (Burke \& Bruford, 1987; Jeffreys et al., 1987; Gilbert et al., 1990; Andersen \& Nilsson-Tillgren, 1997; Bois et al., 1998a). Shown to exhibit high levels of germline mutation rates (up to 15\% per gamete) (Vergnaud et al., 1991), minisatellites have thus been successfully utilised as a tool for monitoring DNA mutation following exposure to environmental doses of mutagenic agents in both human (Dubrova et al., 1996, 1997, 2002; Kodaira et al., 1995, 2004; Satoh et al., 1996; Livshits et al., 2001; Kiuru et al., 2003), and animal populations (Yauk \& Quinn, 1996; Yauk et al., 2000). Indeed such innovation was instigated by Dubrova et al. in 1993 whose pioneering paper implemented the use of previously classified hypervariable minisatellite loci, before reclassification to ESTR loci, to demonstrate a significant increase in the germline mutation rate of offspring following acute parental exposure to 0.5 and $1 \mathrm{~Gy}$ of $\gamma$-rays in mice (Dubrova et al., 1993). Although still applying a pedigree-based approach, the frequency in which mutations are induced following irradiation was calculated using substantially reduced numbers of animals ( 250$)$ when compared to traditional approaches ( 1000 's). Two additional studies similarly employed what later transpired to also be ESTR loci to illustrate a statistically significant increase in the induction of germline mutations at the Ms6-hm hypervariable mouse minisatellite locus following parental exposure at up to $3 \mathrm{~Gy}$ y-rays (Sadamoto et al., 1994; Fan et 
al., 1995). Minisatellite loci have however been applied to the analysis of Herring gulls inhabiting a widely industrialised region of Hamilton harbour (Yauk \& Quinn, 1996). Herring gulls residing within Hamilton harbour presented statistically significant differences in minisatellite mutation rates when compared to gulls from three seemingly non-industrialised control rural areas.

Although it has since been demonstrated that these studies actually employed ESTR loci as opposed to minisatellites, such an extended analysis initially confirmed that hypervariable minisatellite loci can be used successfully as an efficient biomarker in the analysis of genetic damage in germline cells. Consequently, the unstable GC-rich minisatellite loci were thereby implemented in the analysis of mutation induction in human populations following chronic low-dose exposure to environmental mutagens (Kodaira et al., 1995; Dubrova et al., 1996, 1997, 2002; Satoh et al., 1996; Kodaira et al., 2004). Indeed, unstable GC-rich minisatellite loci were initially implemented following the Chernobyl incident, to analyse the germline mutation rates of Belarusian families inhabiting a rural area of the Mogilev district heavily affected by the incident's radioactive fallout. An initial 2 -fold increase in minisatellite mutation rate was observed within families exposed to high levels of caesium-137 surface contamination (Dubrova et al., 1996). Yet insufficient evidence was presented to dispel concerns that the increased mutation rates were instead attributed to sampling errors, such as the use of Caucasian British families as the control families. In an attempt to authenticate the original findings, several follow-up studies were produced (Dubrova et al., 1997, 2002). Firstly, Dubrova and co-authors (1997) extended the analysis to include an additional 48 Belarusian families as well as a further five minisatellite loci. The inclusion of the extra analysis parameters produced an almost identical 1.9-fold increase in mutation rate, yet regardless of this, the control group was still that of British Caucasians. Their third study was then expanded further to study the germline mutation rate in exposed Ukrainian families from other rural areas of Ukraine contaminated with radionuclides, post-Chernobyl (Dubrova et al., 2002). Within this study, however, the control group consisted of matched Ukrainian families. Similarly, a statistically significant 1.6-fold increase in the frequency of minisatellite mutations was observed in those Ukrainian families residing within areas of Ukraine presenting high 
levels of surface radionuclide contamination. In addition, upon re-analysis against the matched Ukrainian control families, the exposed families from Belarus exhibited a statistically significant 1.7-fold increase in germline mutations (Dubrova et al., 2002). Although cumulatively the three individual studies present compelling evidence of elevated minisatellite mutation rates within the Chernobyl area, similar studies of minisatellite mutation rate analysis among children of Chernobyl clean-up workers in Ukraine (Livshits et al., 2001), and Estonia (Kiuru et al., 2003), provide contradicting findings. Interestingly, neither of these two studies reported a statistical difference in mutation rate, despite the Estonian study employing the identical eight minisatellite loci as Dubrova and co-authors $(1997,2002)$. Furthermore, it was calculated that the clean-up workers were exposed to average doses of radiation less than $0.25 \mathrm{~Gy}$ (Pitkevitch et al., 1997), which represents a value lower than the calculated doubling dose of $0.33 \mathrm{~Gy}$ in mice (Dubrova et al., 1998a). Similar non-significant mutation rates were presented in studies of Hiroshima and Nagasaki atomic bomb survivors and their offspring, in which the same eight minisatellite loci were again employed (Kodaira et al., 1995, 2004; Satoh et al., 1996). The detection of alterations in germline mutation rates within human populations is particularly problematic, relying heavily on the extrapolation of estimations from experimental systems relating to the genetic effects of radiation and other mutagens.

Thus, inevitably, the use of animal studies were required in order to elucidate the processes involved in induced tandem repeat mutation, including the mechanisms involved, as well as to resolve the aforementioned inconsistencies in the human investigations.

\subsubsection{Expanded Simple Tandem Repeats (ESTRs)}

As mentioned previously (Chapter 1.14.3), the ESTR regions of mouse DNA containing alleles from the unstable mouse loci Ms6- $\mathrm{hm}$ and $\mathrm{Hm}-2$ were initially classified as hypervariable mouse minisatellites (Kelly et al., 1989; Gibbs et al., 1993; Bois et al., 1998b) and have been implemented to demonstrate a significant increase in the germline mutation rate of offspring following acute parental exposure to 0.5 and $1 \mathrm{~Gy}$ of $\gamma$-rays in mice (Dubrova et al., 1993). However, it has subsequently transpired that the loci are present only in rodent DNA and thus were renamed as expanded simple 
tandem repeat (ESTR) loci to distinguish them from what is deemed 'true' minisatellites in the mouse genome (Kelly et al., 1989; Gibbs et al., 1993; Bois et al., 1998b). The confusion originally stemmed from ESTR loci exhibiting exceedingly high rates of spontaneous mutation in the paternal germline, similar to that of GC-rich human minisatellites. Yet, the occurrence of spontaneous mutations is extremely rare at human minisatellite loci owing to a high level of somatic tissue stability (Jeffreys et al., 1988, 1994). In contrast, ESTR loci provide equally high somatic cell instability (Kelly et al., 1989; Gibbs et al., 1993; Hardwick et al., 2009), similar to that exhibited by microsatellites.

Expanded simple tandem repeats consist of long ( $>1 \mathrm{~kb})$, homologous, non-coding sequences comprised of short tandem repeat arrays (4-10 bp) (Kelly et al., 1989; Gibbs et al., 1993; Bois et al., 1998b). The complexity of the repeat units can also be used to distinguish between loci. Whilst ESTRs comprise a sole repeat unit array, this is not the case for minisatellite loci, which exhibit a large proportion of variant repeats. Similarly, the single repeat unit array contained in the ESTR is generally found to be smaller than in minisatellite loci (Table 8). Additionally, ESTR repeat unit arrays are also deemed too large to be microsatellite loci (Ellegren, 2004). Currently, the only mouse ESTR loci characterised in detail include the Ms6-hm and the Hm-2 (Kelly et al., 1989; Gibbs et al., 1993), both of which originated from highly expanded transcript dispersed repeats in the mouse genome (Kelly, 1994). Ms6- $\mathrm{hm}$ and $\mathrm{Hm}-2$ expanded from independent sequences representative of long terminal repeats (MT and ORR-1 respectively), which belonged to the Mammalian-apparent long terminal repeat (LTR)-retrotransposon (MaLR) superfamily (Kelly, 1994).

Ms6-hm is the locus most predominantly used owing to its characterisation as the most unstable locus in the mouse genome (Hardwick et al., 2009). However, both represent efficient in vivo biomarkers in the detection of germline mutation induction, expressing mutation rates of between 1.7-3.6\% per gamete (Bois et al., 1998b). Both loci also express similarly high levels of spontaneous somatic mutation with rates of up to $2.8 \%$ and $20 \%$ for Ms6-hm and Hm-2, respectively (Kelly et al., 1989; Gibbs et al., 1993). Originally detected as a hypervariable locus used in DNA fingerprinting laboratory mice (Jeffreys et al., 1987), the Ms6-hm locus consists of (GGGCA) 
pentamer repeats and is mapped on chromosome 4. The length of the GGGCA repeat however varies between the different strains of mice, although most alleles contain more than 400 repeat units (Kelly et al., 1989; Gibbs et al., 1993). The Hm-2 locus, on the other hand, consists of (GGCA) $n$ and is mapped on chromosome 9 (Kelly et al., 1989), and shows sequence similarity to that of the (GGCAGA) $)_{n}$ repeat array exhibited by the MMS10 repeat family (Bois et al., 1998b).

Following the successful application of ESTR loci as an analysis tool in the evaluation of germline genetic effects (Dubrova et al., 1993; Sadamoto et al., 1994; Fan et al., 1995), the majority of studies investigating germline mutation induction have implemented the use of unstable mouse ESTR loci. The high frequencies of spontaneous and induced mutations at mouse ESTR loci allows the analysis of mutation induction to be conducted at much lower doses of exposure than those previously accessible with the standard approaches. Similarly, because germline mutation induction at ESTR loci typically ranges between two- to four-fold increases, they can be evaluated using much smaller numbers of animals (Table 9) (Dubrova et al., 1998a).

\subsection{Doubling Dose}

Such a significant improvement in sensitivity, compared to the more traditional approaches, provided a more robust estimate of the doubling dose without the need for extrapolating data from high doses of radiation. More importantly though is that the doubling dose attained using ESTR loci is exceedingly similar to estimates previously acquired using the more traditional approaches of mutation detection in mice, specifically the SLT (Russell 7-locus test) (0.34 Gy) (Searle, 1974). A robust linear dose-response curve was generated following a dose range of 0-1 Gy acute exposure to X-rays in pre-meiotic spermatogonia (Figure 6), culminating in an estimated doubling dose for ESTR mutation of 0.33 Gy (Dubrova et al., 1998a). The doubling dose is used in animal test systems to denote the dose of radiation capable of inducing a mutation frequency corresponding to twice the overall spontaneous mutation frequency per generation (Selby, 1998). Likewise, it can also signify an assay's relative sensitivity in times of inter-assay comparisons (Somers, 2006). Owing to the SLT's previous standing as the most efficient method with which to score protein-coding gene mutations in mice (Searle, 1974), the similarities in doubling doses are of 
significant importance, implying a potential correlation between mutations at tandem repeat DNA and those at coding sequences. These studies taken in conjunction therefore demonstrate a correlation between mutations in tandem repeat sites and other mutation assays insofar as an increase in tandem repeat mutations causes an identical increase in mutation frequency at other sites.

Table 9. Doubling dose estimates

\begin{tabular}{|l|l|l|l|l|}
\hline Method & $\begin{array}{l}\text { Spontaneous } \\
\text { mutation } \\
\text { rate }\end{array}$ & $\begin{array}{l}\text { Total number } \\
\text { of animals } \\
\text { tested }\end{array}$ & $\begin{array}{l}\text { Exposure } \\
\text { doses used } \\
(\mathbf{G y})\end{array}$ & Doubling dose (Gy) \\
\hline $\begin{array}{l}\text { Russell 7- } \\
\text { locus }\end{array}$ & $7.95 \times 10^{-6}$ & $1,051,869$ & $3,6,6.7$ & $\begin{array}{l}0.34(95 \%, \mathrm{Cl}: 0.22-0.50) \\
0.38( \pm 0.05)^{*}\end{array}$ \\
\hline $\begin{array}{l}\text { Dominant } \\
\text { Lethal }\end{array}$ & $8.11 \times 10^{-6}$ & 225,017 & 6,12 & $0.17(95 \%, \mathrm{Cl}: 0.00-0.59)$ \\
\hline ESTR (2 loci) & $5.56 \times 10^{-2}$ & 252 & $0.5,1$ & $\begin{array}{l}0.33(95 \%, \mathrm{Cl}: 0.06-0.75) \\
0.41( \pm 0.11)^{*}\end{array}$ \\
\hline
\end{tabular}

Comparison of the doubling dose estimates for acute exposure to ionising radiation of mice spermatogonia (Table adapted from Dubrova et al., (1998a). * re-assessed value (Dubrova, 2005).

\subsection{ESTR Mutation Detection Studies}

Subsequently numerous studies have shown that ESTR loci can be implemented successfully to provide an exceedingly sensitive system through which germline and somatic mutation rates can be analysed following exposure to: (a) ionising radiation (Dubrova et al., 1993, 1998a , 2000, 2000a; Barber et al., 2002, 2006; Dubrova \& Plumb, 2002; Yauk et al., 2002; Dubrova, 2005; Mughal et al., 2012). Of particular note is the study by Mughal et al. (2012) in which paternal exposure to doses of acute $\gamma$-rays as low as 50 cGy resulted in significant $\sim 2$-fold increase in the frequency of ESTR mutation. (b) Chemical mutagens (Vilarino-Guell et al., 2003), which included the treatment of male mice with several doses of known mutagenic alkylating agents: ethylnitrosourea (ENU), isopropyl methanesulfonate (iPMS), or alternatively the topoisomerase II inhibitor etoposide and (c) Anticancer drugs (Barber et al., 2000; Glen et al., 2008). It should be noted however that the study by Barber and co-authors (2000) failed to observe any change on ESTR mutation rates at any stage during spermatogenesis at which the cisplatin was administered. These findings are somewhat unexplainable considering cisplatin is widely known to propagate a host of DNA-damaging events, notably chromosomal aberrations occurring in mouse spermatocytes and spermatogonial stem cells. The dose of cisplatin that was 
administered $(10 \mathrm{mg} / \mathrm{kg})$ is perhaps notable in that similar studies employing both the dominant lethal and specific locus tests also failed to display any effect at such a dose (Katoh et al., 1990; Witt \& Bishop, 1996). One hypothesis for these negative results is that of potential cell killing inadvertently affecting the amount of mutants available for detection (Barber et al., 2000), owing to the administered dose of cisplatin (10 mg/kg) being twenty times that of the doubling dose for chromosome abnormalities ( 0.5 $\mathrm{mg} / \mathrm{kg}$ ) (Somers, 2006). Glen and co-authors (2008) also utilised ESTR loci alongside a novel methodology known as single-molecule PCR (reviewed later in Chapter 1.14.4.1) to analyse the rate of male germline mutation induction for four commonly used anticancer drugs: bleomycin, cyclophosphamide, mitomycin $\mathrm{C}$ and procarbazine. Each chemotherapy drug was applied at clinically relevant doses for humans, which were calculated in accordance to Food and Drug administration guidelines. In addition, ENU was also administered as an assay positive control following previous reports of significant increases in mutation frequencies (Vilarino-Guell et al., 2003). Significant increases in sperm ESTR mutation frequencies were presented for each of the four anticancer drugs (Glen et al., 2008).

Such sensitivity of ESTR loci signified a potential useful tool for studies of environmental pollution thus, lastly but of equal importance, is their implementation in the analysis of (d) environmental mutagens, including ambient urban/industrial air pollution (Somers et al., 2002, 2004; Yauk et al., 2008; Zhou et al., 2009), diesel exhaust particles (Ritz et al., 2011) and sidestream tobacco smoke (Yauk et al., 2007; Marchetti et al., 2011). A similar detection of de novo mutations in ESTRs has also been observed in vitro, signifying a correlation between the mutation responses of cultured embryonic fibroblasts and germ cells and somatic cells in vivo following treatment with chemical mutagens (Polyzos et al., 2006a, 2006b).

There are several benefits to the use of ESTR loci in studies of this kind: whilst enabling the identification of direct parental exposure to mutagenic agents, the use of ESTR loci further facilitates the ability to identify the transmission of radiation-induced somatic genome mutations through the germline to first generation $\left(F_{1}\right)$ offspring (Dubrova et al., 2000). Indeed, significant increases in mutation rate were detected at ESTR loci in first generation $\left(F_{1}\right)$ offspring following parental exposure to ionising radiation 
(Dubrova et al., 2000; Mughal et al., 2012), anticancer drugs (Glen \& Dubrova, 2012), and chemical mutagens (Dubrova et al., 2008). Continuation studies confirmed those previous findings, while also expanding the analysis to illustrate that ESTR mutation rates remain elevated in three separate unexposed second generation $\left(F_{2}\right)$ offspring (Barber et al., 2002), and that ESTR transgenerational genomic instability also manifests in somatic tissues (Barber et al., 2006). In contrast, several studies have reported that maternal in utero exposure fails to illicit any effect on transgenerational instability (Barber et al., 2009; Abouzeid Ali et al., 2012). Likewise, the frequency of ESTR mutation of unexposed first generation offspring remained similar to control levels following the administration of a parental methyl-donor deficient diet (Voutounou et al., 2012).

\subsection{Mechanisms of Mutation at ESTR Loci}

Although a mutation response at ESTR loci consists of either an insertion or deletion of repeat sequence, translating to alterations in the allele length, the mechanisms through which this transpires remain poorly understood. Following differences in somatic stability, it was theorised that ESTR mutations arise through a different mechanistic process to that of unstable GC-rich human minisatellites (Kelly et al., 1989, 1991; Gibbs et al., 1993; Bois et al., 1998b). The high frequency of germline mutations at human minisatellites arise through complex meiotic recombination events, however ESTR loci display equal levels of somatic mutation induction, while human minisatellites do not (Jeffreys et al., 1988, 1994). Moreover, a study by Barber et al. (2000) provided further confirmation that increased mutation induction at ESTR loci are not caused by genome-wide elevations in meiotic recombination. Since meiotic recombination is often connected with chromosomal crossover, the authors measured both crossover frequency and germline ESTR mutation frequency following acute exposure to $1 \mathrm{~Gy} \mathrm{X}$-rays or $10 \mathrm{mg} / \mathrm{kg}$ of cisplatin to ascertain whether mutation induction at ESTR loci is attributed to meiotic recombination (Barber et al., 2000). Cisplatin is a commonly used anticancer drug which is known to produce a variety of DNA adducts, particularly 1, 2-intrastrand meiotic crossovers (Hanneman et al., 1997). Although the authors detected a 3-fold increase in ESTR mutation frequency after examining 25 sites located on six separate chromosomes when compared to the controls, no overall alteration in crossover frequencies was identified. This therefore 
suggests that ESTR mutation occurs in the absence of meiotic-specific DSBs and meiotic recombination (Barber et al., 2000).

Further mechanistic insights of ESTR mutation induction were provided following exposure to both ionising radiation (Sadamoto et al., 1994; Fan et al., 1995; Dubrova et al., 1998a, 1998b, 2000a; Yauk et al., 2002) and chemical mutagens (Vilarino-Guell et al., 2003; Glen et al., 2008). Mutation spectra data generated from studies suggest that both induced and spontaneous ESTR mutations in germline or somatic tissues arise through similar mechanisms (Yauk et al., 2002; Hardwick et al., 2009). Moreover, these data further suggest that the detected increases in ESTR mutation rates are not the direct result of mutagens targeting ESTR loci. It was therefore theorised that ESTR mutations arise through a replication or repair based process involving DNA polymerase slippage during DNA replication, or through DNA polymerase pausing to repair the DNA (Yauk et al., 2002; Hardwick et al., 2009). An explanation that was supported by data compiled from the use of DNA repair-deficient male mice (Barber et al., 2004).

The high spontaneous mutation rates exhibited by ESTR loci are potentially linked to either the size of their repeat length (up to 4000 base-pairs) (Bois et al., 1998a, 1998b), or the formation of hairpin structures among the repeat region of ESTR loci during DNA replication, repair and recombination (Weitzmann et al., 1998; Fukuda et al., 2002). A hypothesis supported by data in which observed mutation rates correlate to the size of ESTR allele (Bois et al., 2001; Dubrova, 2005). The representation of these aforementioned hairpin structures instigate DNA polymerase stalling/replication pausing and essentially promote the occurrence of DNA polymerase slippage events (Kang et al., 1995). DNA polymerase stalling ensues following the delayed repair of DNA damage thus, in an attempt to fix DNA damage while crossing an ESTR locus, a polymerase slippage event occurs causing the formation of hairpins among repeat sequences in the ESTR arrays. Upon replication re-initiation these are subsequently transformed into an insertion or deletion of sequence and are detected as length changes (Figures 4 \& 5) (Kang et al., 1995). Indeed, a replication-based process similar to mechanisms evoked in microsatellite instability (Ellegren, 2004) represents the most feasible method through which ESTR mutations occur. Furthermore, the proposed 
replication-based method also provides a conceivable explanation regarding how mutation frequencies remain elevated at ESTR loci across multiple generations (Dubrova et al., 2000; Barber et al., 2002, 2006). 


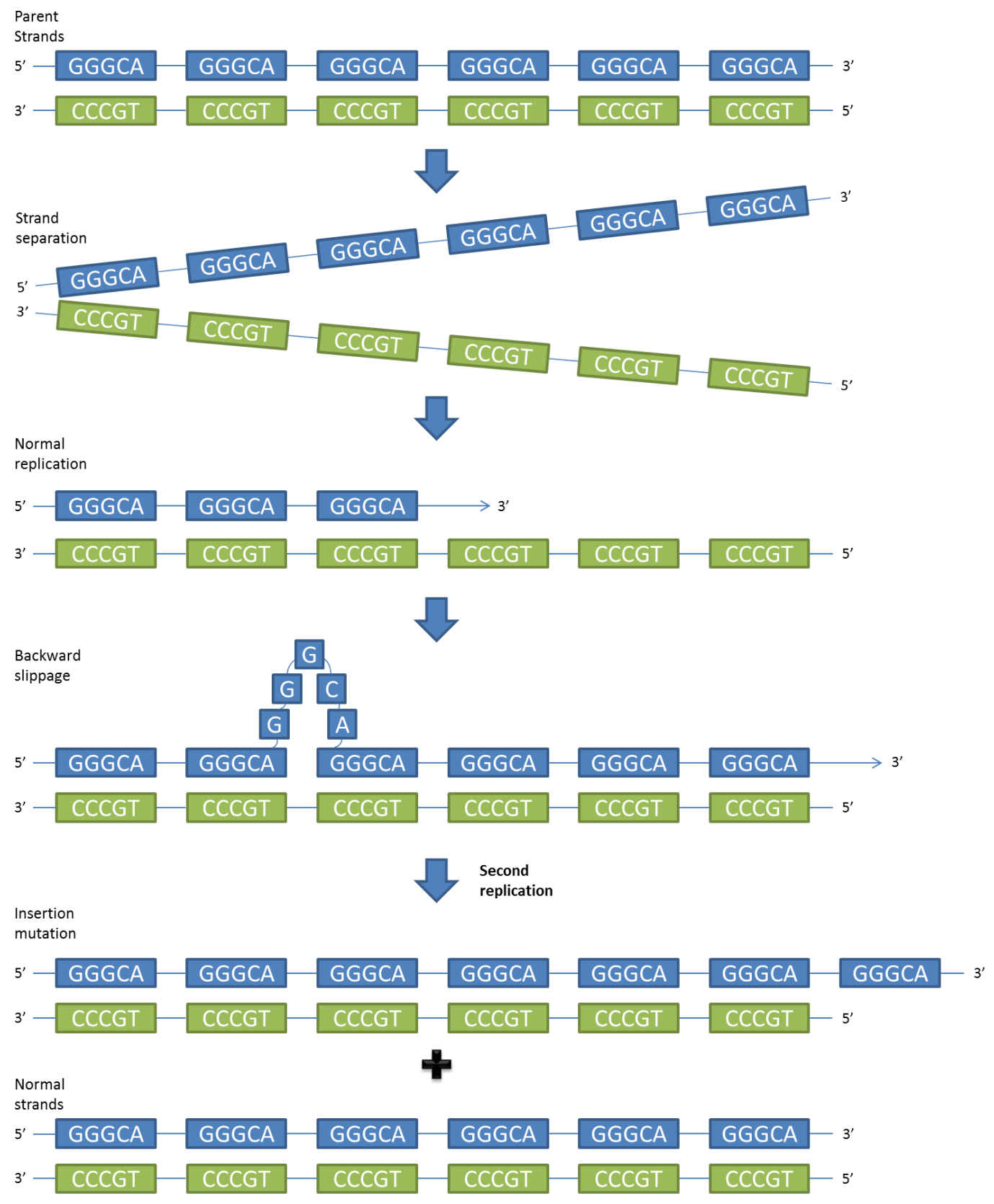

Figure 4. Diagrammatic representation of an insertion mutation caused by replication slippage. Strand mispairing (DNA polymerase slippage) during DNA replication on the daughter strand (backward slippage) results in an insertion mutation. Diagram modified from (Kang et al., 1995; Ellegren, 2004). 


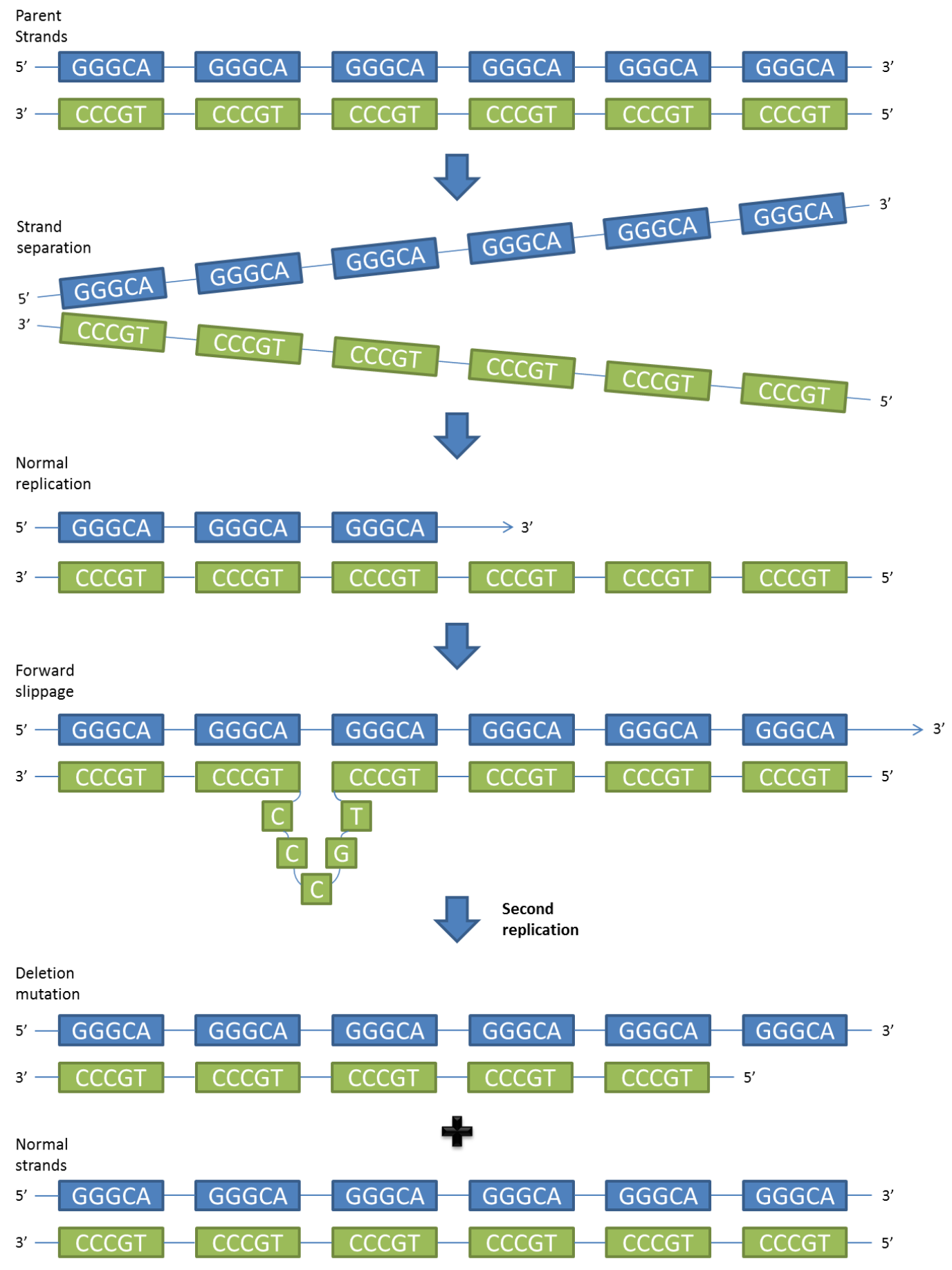

Figure 5. Diagrammatic representation of a deletion mutation caused by replication slippage. Strand mispairing (DNA polymerase slippage) during DNA replication in the parent strand (forward slippage) results in a deletion mutation. Diagram modified from (Ellegren, 2004). 
In addition to the process through which ESTR mutations occur, the stage of development at which ESTR mutations arise is also of equal mechanistic importance. Data from numerous studies, analysing the ESTR mutation rate following acute irradiation to X-rays at various stages of spermatogenesis, have shown that rates are significantly elevated only in samples taken at 4,5 , or 6 and above weeks, posttreatment (Dubrova et al., 1998a, 1998b; Barber et al., 2000). These time points represent spermatogonia derived from mid and early pachytene, type $B$ spermatogonia and $A_{s}$ spermatogonia, which are indicative of pre-meiotic spermatogonia (Searle, 1974). In contrast, no measurable alterations in the frequency of ESTR mutations were exhibited in post-meiotic spermatids procured from nondividing sperm cells of irradiated adult mice analysed either 1 week (Barber et al., 2009) or 3 weeks (Dubrova et al., 1998a, 1998b; Barber et al., 2000) after exposure (Figure 6). A collective analysis of these data therefore indicates a proclivity for all radiation-induced ESTR mutations to arise at every stage of spermatogenesis preceding metaphase I (Barber et al., 2000). In addition to induced mouse germline mutations at the ESTR loci, a study by Hardwick and co-authors (2009) investigated the spontaneous mutation process through the measurement of age-related changes in mutation frequencies for male mice aged 12, 26, 48 and 96 weeks. The authors highlighted an abundance of ESTR mutations accumulating in the sperm of older male mice, whereby the number of age-related ESTR mutations correlate with that of cell divisions occurring in the tissue. Considering that spermatogenesis is a continual process throughout adulthood and possesses an exceedingly high proliferation activity of stem cells (de Rooij \& Russell, 2000), these findings seem to indicate that both induced and spontaneous ESTR mutation rates correlate to the proliferation rate of the tissues, whereby the age-related increases in ESTR mutation frequency can be explained by an accumulation of replication-driven mutations. Consequently, tissues which manifest a low mitotic index therein present lower ESTR mutation rates. Indeed, similar observations also correspond to induced and spontaneous ESTR mutations in somatic cells. ESTR mutation rates in DNA samples taken from bone marrow and brain cells were similarly measured for age-related changes (Hardwick et al., 2009), in addition to those induced by radiation (Barber et al., 2009). The relatively 
small changes in mutation rate exhibited in bone marrow cells following acute exposure to X-rays (Barber et al., 2009), coupled with the significant elevations correlating with age, again correspond to the cellular replication proficiency of the tissue, given that the percentage of actively dividing stem cells in mouse bone marrow is very low ( 9.1/10 5 cells in BALB/c mice) (Muller-Sieburg \& Riblet, 1996). Similarly, ESTR mutation frequencies calculated from the brains of males irradiated in utero were significantly elevated (Barber et al., 2009), whilst no discernible change in mutation frequency appeared in non-proliferating adult brains either induced or in conjunction with aging (Hardwick et al., 2009). Since the mitotic proliferation capabilities in an adult brain are low to non-existent (Gross, 2000), with brain cells only undergoing mitotic proliferation in utero, these data further signify such an association.

Overall, these findings taken together constitute further evidence that ESTR mutations arise almost entirely in replication-proficient cells, through a replication dependent mechanism predominantly expected to be replication slippage. 


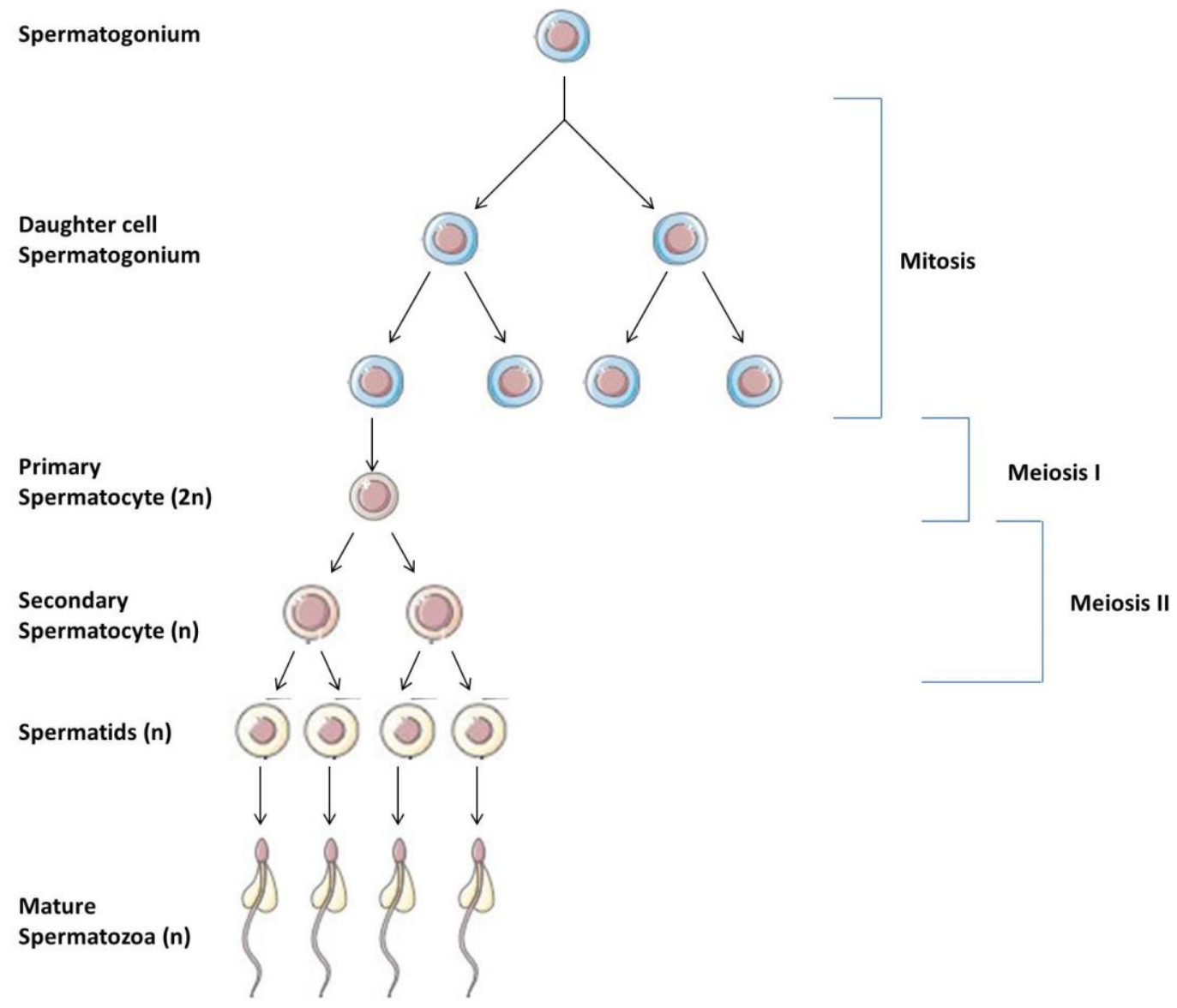

Figure 6. Spermatogenesis. Originating in the males testes, spermatogenesis represents the production of sperm and occurs continuously from puberty onwards (six weeks after birth in mice), taking approximately 35 days. Male germ cells identified as spermatogonia undergo many mitotic cell divisions generating diploid primary spermatocytes. These cells then undergo meiosis I producing secondary spermatocytes. Each secondary spermatocyte undergoes meiosis II to produce haploid spermatids which subsequently mature into spermatozoa (reviewed in Cooke \& Saunders, (2002)). Figure adapted from Berne et al., (2005), 2n denotes diploid cells while $\mathrm{n}$ denotes those which are haploid. 


\subsubsection{Methodology}

Although most experimental data regarding mutagen-induced tandem repeat mutations in the germline were generated using ESTR loci, the early studies relied upon a pedigree-based approach to determine in vivo germline mutation rates in offspring of test males (Dubrova et al., 1993, 1998a, 1998b, 2000a; Vilarino-Guell et al., 2003). Pedigree-based studies however do not represent the most efficient method of mutation detection, especially in human minisatellite studies where mutation rates are in the region of $1 \%$ per gamete (Bois \& Jeffreys, 1999). Similar limitations persist in animal studies, including extended experimental timescales through mating and birthing processes and, although drastically reduced, the issue of using an excessive number of animals still continued. These issues were overcome through the progressive development of PCR methodologies, initially through small-pool PCR (SPPCR) (Jeffreys et al., 1994) and later single-molecule PCR (SM-PCR) (Yauk et al., 2002). Such methodological advancements in mutation screening provide more robust estimations regarding the effect of mutagens on individual mutation rates. Both supply the capability to screen an unrestricted amount of novel germline and somatic alleles, taken directly from genomic DNA of the same exposed individual in a single experiment. Consequently, the number of animals required to undergo analysis has drastically reduced, such that mutation frequencies can now be generated in response to lower exposure doses (Dubrova et al., 2000a; Glen et al., 2008; Mughal et al., 2012), and by using just five animals per exposure parameter (Yauk et al., 2002).

\subsubsection{PCR Methodologies}

Such improved characteristics are accomplished through the bulk dilution of genomic DNA (Jeffreys et al., 1994; Yauk et al., 2002). Initially, SP-PCR amplifies multiple aliquots, each containing approximately 10-100 individual progenitor molecules (Jeffreys et al., 1994). While SP-PCR has been used successfully in attaining several reliable estimates of mutation rates in the regions of $10^{-3}$ and $10^{-4}$, the majority of mutant alleles present indistinguishable length changes which, in the presence of multiple progenitor molecules become difficult to quantify (Jeffreys et al., 1994). Similarly, the existence of PCR artefacts resulting from PCR amplification errors further hinder the detection of genuine mutants expressing such low mutation rates. 
Further refinement of the methodology culminated in the production of singlemolecule PCR (Yauk et al., 2002), whereby multiple reactions are established, each containing on average at least one amplifiable ESTR molecule. Although this process requires the preparation of more aliquots than SP-PCR, the amplification of a single progenitor molecule is advantageous in that de novo mutant ESTR alleles are more readily detected. Moreover, data obtained from ESTR analysis have been shown to correlate with those generated by the previous techniques (Dubrova et al., 1998a), thus proving that investigation through ESTR loci provides a reliable representation of mutation induction throughout the genome.

Initially implemented by Yauk and co-authors in 2002, SM-PCR has since been used in conjunction with ESTRs to directly measure in vivo mutation frequencies in both germline and somatic tissues following exposure to ionising radiation (Abouzeid Ali et al., 2012; Mughal et al., 2012), and anticancer drugs (Glen et al., 2008). Therefore, through implementing this system within the current study, we have evaluated the in vivo effects of exposure to extremely low-frequency $(50 \mathrm{~Hz})$ magnetic fields on mutation induction in the germline and somatic tissues of $B A L B / C \times C B A / C a F_{1}$ hybrid male mice. 


\subsection{Aims and Objectives}

The Effects of ELF-MF on Mutation Induction

Given the profound differences in the mutagenic data generated following ELF-MF irradiation, this study was specifically designed to evaluate the current poorly understood genetic effects of ELF-MF exposure on ESTR mutation induction in vivo. By establishing the effects of ELF-MF doses currently within the accepted reference limits for a $50 \mathrm{~Hz}$ magnetic field (International Commission on Non-lonizing Radiation Protection, 2010), in both the mouse germline (sperm) and somatic tissues (blood) of directly exposed male mice, the data generated can provide an experimentally based assessment of the effects of ELF-MF exposure on mutation induction.

Additionally, the pattern of ESTR mutation induction will be analysed in the germline and somatic tissues of male mice exposed to $1 \mathrm{~Gy}$ acute $\mathrm{X}$-rays, and the mutagenic effects compared against those of ELF-MF exposure.

\section{The Effects of ELF-MF and 1 Gy Acute X-ray Exposure on ESTR Mutation Spectrum}

This study aims to establish whether ELF-MF or 1 Gy acute X-rays alters the spectra of ESTR mutation in the mouse germline and somatic tissues. Such analysis can provide further evidence into the mechanisms involved in the generation of induced and spontaneous mutations at these loci.

The Effects of ELF-MF and 1 Gy Acute X-ray Exposure on the Genome-wide Pattern of Gene Expression

Finally, this study aims to analyse and compare the genome-wide pattern of gene expression in the ELF-MF and X-ray irradiated males. Acting as a pilot study, it aims to validate the results obtained by the ESTR assay, while also providing a broader insight into the effects of long-term acute exposure to ionising radiation. 


\section{Materials and Methods}

\subsection{Mice}

$\mathrm{CBA} / \mathrm{Ca}$ males were mated with $\mathrm{BALB} / \mathrm{c}$ females to produce a BALB/C $\times \mathrm{CBA} / \mathrm{Ca}$ hybrid male mice $F_{1}$ generation (Harlan, Bicester, UK). The BALB/C $\times C B A / C a F_{1}$ hybrid males were irradiated at 7 weeks old at the Public Health England, Centre for Radiation, Chilton, UK. Somatic and germline tissues were collected from the hybrid males 12 weeks post-irradiation at Public Health England, UK.

Prior to, and following exposures all mice were maintained in conventional housing with a light/dark cycle of 12/12 hours. Rooms were maintained at $21 \pm 2{ }^{\circ} \mathrm{C}, 45-56 \%$ relative humidity with approximately 20 air changes per hour.

All animal procedures were approved by the Local Ethical Review Committee and performed under the Animals (scientific procedures) Act 1986.

\subsection{Exposure Procedure}

\subsubsection{Magnetic Field Exposure System}

The magnetic fields were generated using an exposure system (Figure 8) consisting of a function generator (Hewlett Packard 3324A), a power amplifier (Kepco BOP-50-2M) and a pair of horizontal Helmholtz coils. The horizontal coils (overall diameter of 54 $\mathrm{cm}$ ) and vertical coils (overall diameter of $99 \mathrm{~cm}$ ) were manufactured by the Clarendon Laboratory, (University of Oxford, UK.). Each coil consisted of 150 turns of 20 standard wire gauge (equivalent to $0.914 \mathrm{~mm}$ ) copper wire wound on a frame made from Tufnol resin. An earthed screen was wound around all coils to prevent stray electric fields. Upon field initiation, the required field intensity was stabilised within 60-120 seconds (Figure 7).

The magnetic field uniformity was measured using a 3-axis fluxgate magnetometer (MAG-03 MC, Bartington Instruments, UK) connected to a digital voltmeter (Solatron SL 6071). The Bartington fluxgate magnetometer was calibrated annually using equipment traceable to national standards.

A personal computer running custom-made software ensured the pre-set field intensity (flux density) was maintained at $\pm 5 \%$ of the nominal at all times; the flux 
density for each axis of the field was also recorded at approximately twenty second intervals and any deviation then corrected. Mapping revealed that the generated field was homogenous to $\pm 5 \%$ within the centre of the coils occupied by the mice.
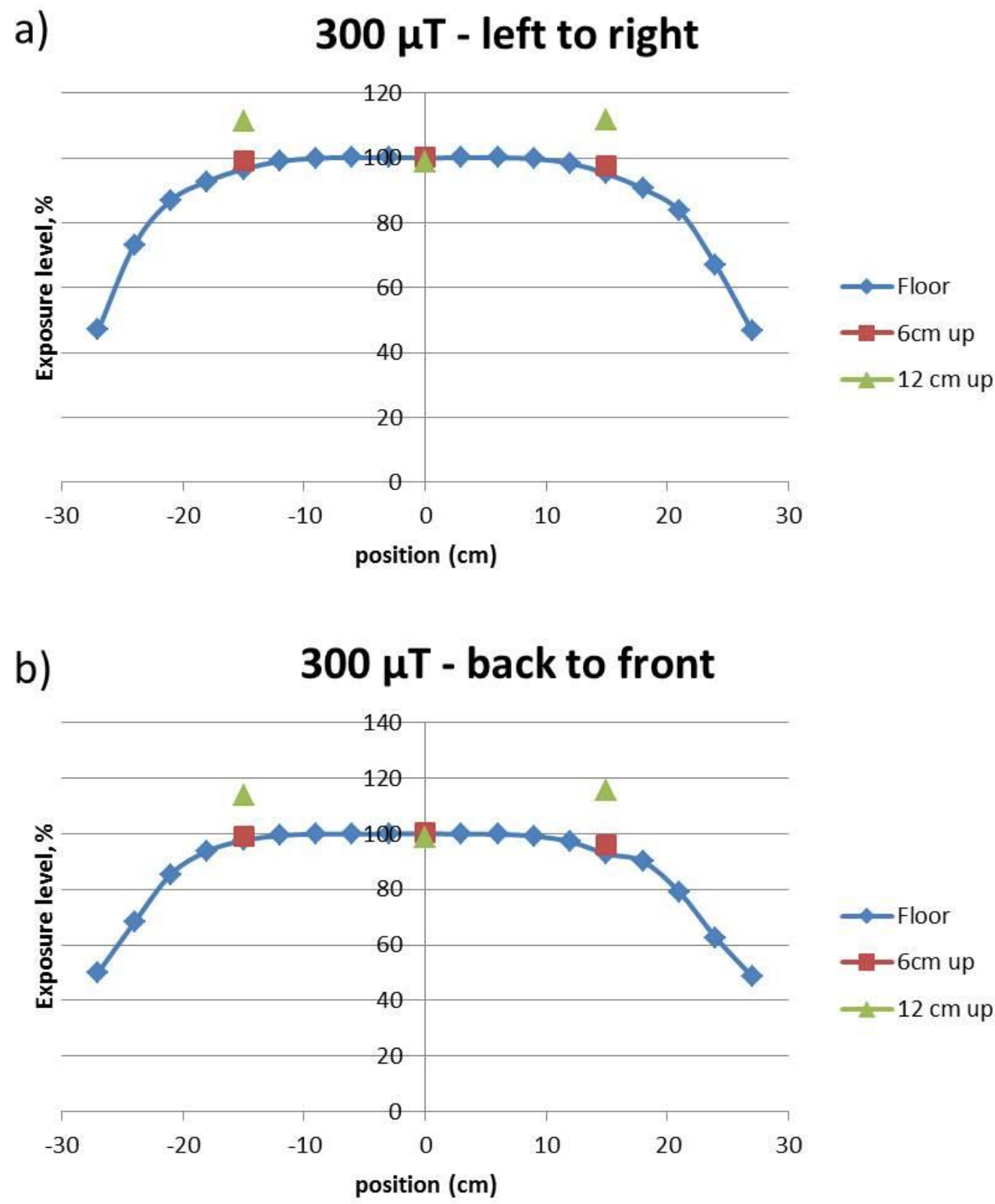

Figure 7. Field uniformity measurement. $50 \mathrm{~Hz}$ Extremely low-frequency magnetic field uniformity for an exposure dose of $300 \mu \mathrm{T}$. The cages were placed $50 \mathrm{~cm}$ from the base of the coil system ensuring that mice were centred in the most homogenous part of the field (plotted area ' 0 '). Field measurements were also taken at 6 and $12 \mathrm{~cm}$ above ground level. a) Field uniformity measured from left to right. b) Field uniformity measured from back to front. 
During exposures, groups of five mice were housed in square, acrylic cages ( $30 \times 30 \times$ $14 \mathrm{~cm}$; Vet Tech Solutions Ltd, Congleton, UK). The cages were placed $50 \mathrm{~cm}$ from the base of the coil system ensuring that mice were centred in the most homogenous part of the field. Each cage was provided with commercial, dust-free bedding material and with free access to water and standard diet (SDS RM3; Lillico, UK). Mice were placed within the cages immediately before exposure and returned to their home cages after exposure. Animals were observed remotely during exposure using CCTV.

\subsubsection{ELF-MF Exposure}

Exposures were performed using a vertical $50 \mathrm{~Hz}$ magnetic field at 10, 100 or $300 \mu \mathrm{T}$ for 2 or 15 hours; the static (or DC) field was maintained at $42.7 \mu \mathrm{T}$, the average value of the static field in the laboratory containing the exposure system. A further two groups of 5 sham-exposed control mice were treated identically but without exposure to $50 \mathrm{~Hz}$ magnetic fields.

Treatments commenced at 09:30 for the 2 hour exposure periods and at 17:00 for the 15 hour exposures. Sham exposed control animals were treated identically but without explicit $50 \mathrm{~Hz}$ magnetic field exposure. The average background $50 \mathrm{~Hz}$ magnetic was less than $0.01 \mu \mathrm{T}$ when measured over 24 hours using an EMDEX II magnetic field dose meter.

\subsubsection{X-ray Irradiation}

Groups of five mice were given whole-body, acute irradiation of $1 \mathrm{~Gy}$ of $250 \mathrm{kV}$ X-rays, delivered at a dose rate of $0.5 \mathrm{~Gy} \mathrm{~min}^{-1}$ as a positive control using a commercial, small animal exposure system (HS System; AGO X-rays Ltd, Aldermaston, UK). During exposure, mice were group housed in corrugated-plastic cages (31 x $27 \times 14.5 \mathrm{~cm})$. One further group of 5 sham-exposed control animals were again treated identically, but without exposure to the whole-body, acute irradiation $1 \mathrm{~Gy}$ of X-rays. 


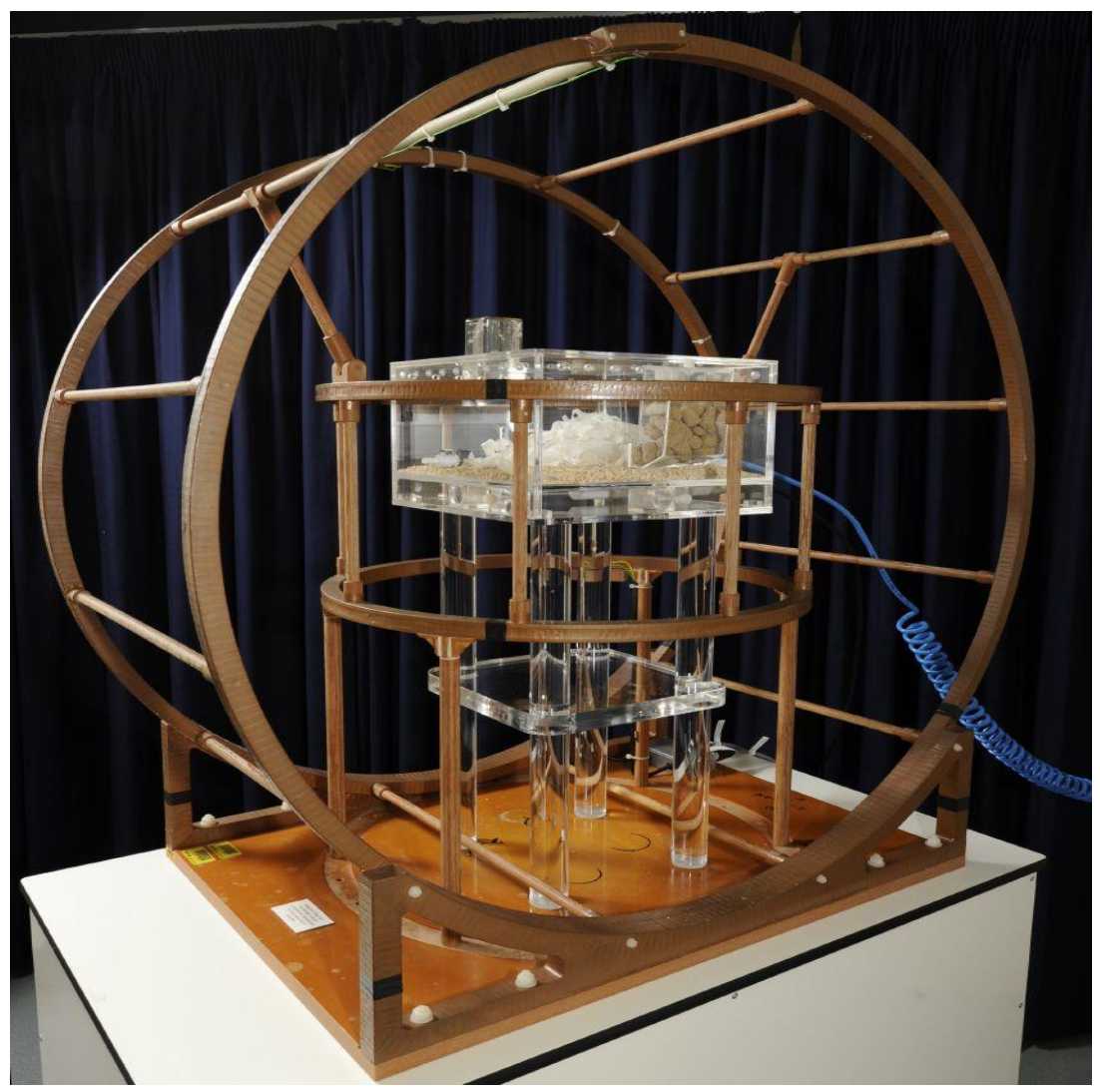

Figure 8. Helmholtz Coil Exposure System. The Helmholtz coil exposure system showing one of the acrylic exposure cages containing bedding, food and water.

\subsection{Tissues}

Blood and testes were taken 12 weeks post-irradiation and DNA samples were extracted from both tissues and purified.

Sperm samples are used to estimate the frequency of ESTR mutation induction and were taken at 12 weeks after exposure to ensure that the sperm samples were derived from exposed $A_{s}$ spermatogonia and stem cells (Searle, 1974) (Figure 6), which is necessary since previous studies have illustrated that ESTR mutation is a replicationbased process involving polymerase slippage (Barber et al., 2004, 2009; Shanks et al., 2008; Hardwick et al., 2009). Thus, the mutagenic effects of ELF-MF irradiation should lead to the accumulation of ESTR mutations in tissues with a high mitotic index, such as spermatogonia and stem cells.

In order to establish the genetic effects of ELF-MF exposure on mutation induction within the somatic tissues of mice, DNA samples were extracted from blood samples taken from the same irradiated animals 12 weeks after exposure. 
The lifespan of specific mature blood cell types is relatively short with approximately $1 \%$ of red blood cells being removed each day. Therefore the regeneration and replacement of adult blood cells represents a continuous process throughout life, originating with hematopoietic stem cells (HSC), located mainly in the bone marrow (BM) niche of adult mammals and occurring through a series of progenitor stages. Hematopoietic stem cells are multi-potent and thus exist as the hierarchy of progenitor cells. They possess the ability to differentiate into multi-lineage progenitors and precursors, ultimately facilitating the emergence of more than ten different individual adult blood cell lineages, each of which possesses unique functions. In addition to their role in replenishing individual blood lineages, immortal HSCs also maintain the blood system through the production of extra HSCs through their ability to self-renew (Orkin \& Zon, 2008).

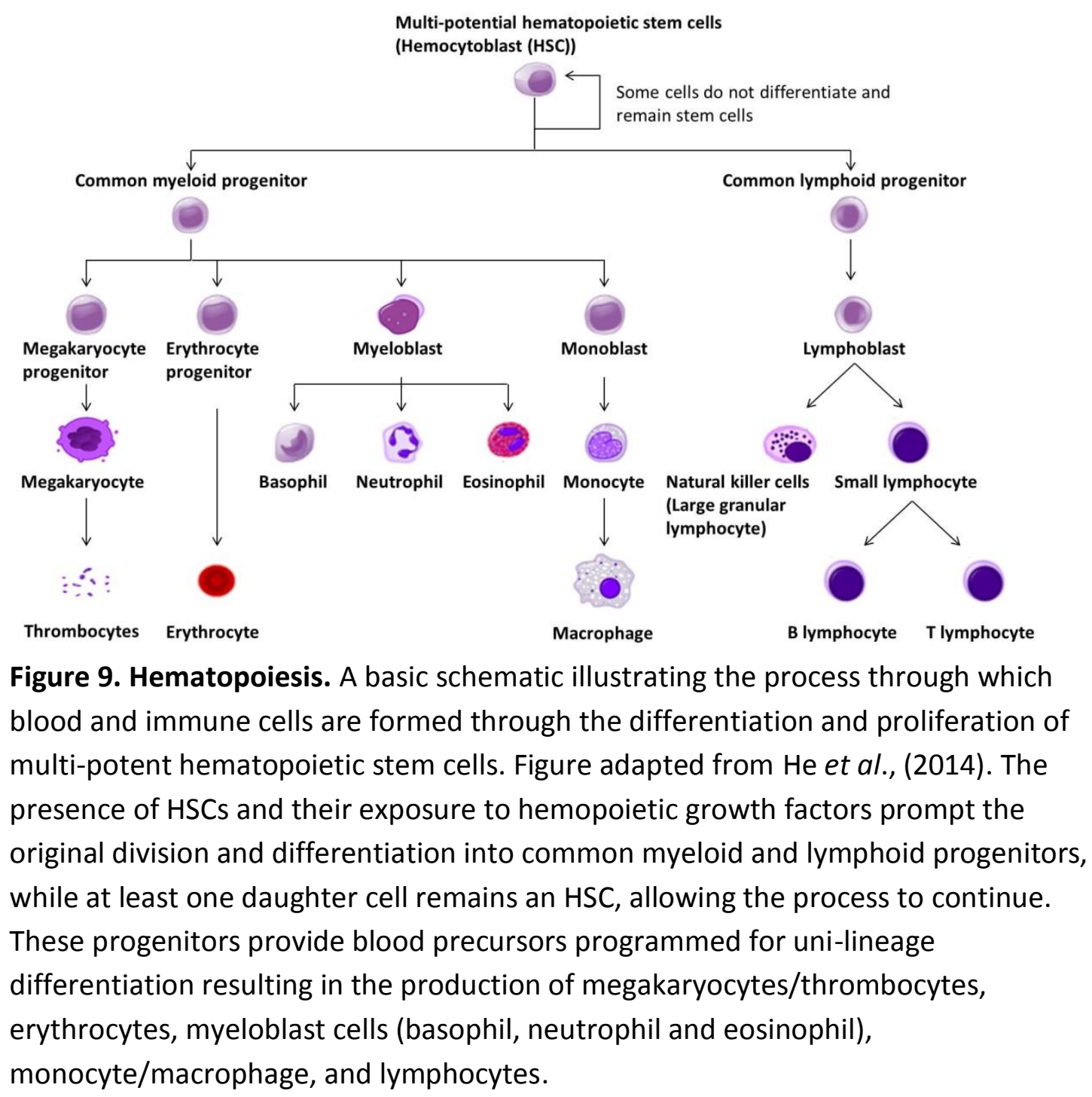




\subsection{DNA Extraction}

All DNA extractions were performed in a category II laminar flow hood (Walker Safety Cabinets Ltd, Glossop, UK) in order to minimise the risk of contamination by foreign DNA molecules.

\subsubsection{Blood}

Blood DNA was extracted using the Wizard Genomic DNA Purification Kit (Promega, Southampton, UK). The blood samples ( $300 \mu \mathrm{L}$ ) were added to $900 \mu \mathrm{L}$ Cell Lysis Solution (Promega, Southampton, UK) and inverted 5-6 times to mix before being left to incubate for 10 minutes at room temperature (RT). The samples were centrifuged (30 seconds, 13,200 revolutions per minute (rpm)), (Eppendorf, Hamburg, Germany) to separate the red blood cells and cell debris from the white blood cells. The supernatant was removed without disturbing the pellet. The process of cell lysis was repeated until the pellet contained only white blood cells (white pellet). Upon generation of the white pellet, the white blood cells were re-suspended at $40 \mathrm{~Hz}$ on the vortex mixer (ZX3, VELP Scientifica, Italy). $300 \mu \mathrm{L}$ of Nuclei Lysis Solution (Promega, Southampton, UK) was added and the samples mixed in order to lyse the white blood cells. If cell clumps were still visible after mixing then the samples were incubated at $37^{\circ} \mathrm{C}$ in a circulating water bath (Grant, Cambridge, UK) until the clumps were disrupted. Although, if the cell clumps remained after an hour then an additional 100 $\mu \mathrm{L}$ Nuclei Lysis Solution was added and the incubation was repeated. Once the cell clumps had dissipated then $100 \mu \mathrm{L}$ Protein Precipitation Solution (Promega, Southampton, UK) was added and the sample mixed on the vortex $(40 \mathrm{~Hz}, 10-20$ seconds). Samples were then centrifuged ( 3 minutes, 13,200 rpm) to remove the proteins. The supernatant was transferred to a new Eppendorf tube containing $300 \mu \mathrm{L}$ single molecule-PCR clean isopropanol and gently mixed. The mixture was then centrifuged ( 3 minutes, 13,200 rpm) to separate the DNA. The supernatant was again removed and the pellet was washed with $300 \mu \mathrm{L} 70 \%$ ethanol before centrifugation occurred again (3 minutes, 13,200 rpm). The supernatant was once more removed and the pellet left to air dry for 15 minutes in the laminar flow hood. The pellet was finally re-suspended in $50 \mu \mathrm{L}$ DNA Rehydration Solution (Promega, Southampton, UK) and the DNA left to rehydrate overnight $\left(4^{\circ} \mathrm{C}\right)$. 


\subsubsection{Sperm}

Sperm DNA was extracted from the testes of the hybrid $F_{1}$ male mice. Each DNA extraction was performed on individual testicles and DNA from both testes combined for each animal. Each testicle was placed in a sterile Petri dish and the excess fat removed before being finely chopped. The disaggregated testicle was mixed with $1 \mathrm{~mL}$ phosphate-buffered saline (PBS) and transferred through a size 80 mesh (Sigma-Aldrich Company Ltd, Poole, UK) into an Eppendorf tube. Then $5 \mu \mathrm{L}$ of the PBS/tissue solution was transferred to a haemocytometer and visualised under a light microscope (Olympus) to determine a sperm to somatic tissue ratio.

The Eppendorf tubes were centrifuged ( 2 minutes, 13,200 rpm) and the supernatant removed. Afterwards, they were centrifuged (30 seconds, 13,200 rpm) and the remaining PBS removed again. Then $1 \mathrm{~mL} 1 \times$ Saline-Sodium Citrate (SSC) was added and the samples mixed on the vortex $(40 \mathrm{~Hz})$ in order to re-suspend the pellet before $10 \mu \mathrm{L}, 10 \%$ Sodium dodecyl Sulphate (SDS), (Fisher Scientific, Loughborough, UK) was added. The samples were centrifuged ( 2 minutes, 13,200 rpm) to produce a pure white pellet. The supernatant was removed and the samples centrifuged again (30 seconds, $13,200 \mathrm{rpm}$ ) and the residual liquid removed. Then $960 \mu \mathrm{L}, 0.2 \times \mathrm{SSC}$ was added and the pellet re-suspended using the vortex mixer $(40 \mathrm{~Hz})$. The samples were once more visualised under the light microscope to ensure that no somatic cells were present. Then $70 \mu \mathrm{L} \beta$-Mercaptoethanol (Sigma-Aldrich Company Ltd, Poole, UK), $100 \mu \mathrm{L}, 10 \%$ SDS and $20 \mu \mathrm{L}, 25 \mathrm{mg} / \mathrm{mL}$ Proteinase K (Sigma-Aldrich Company Ltd, Poole, UK) were added and the tubes incubated at $37^{\circ} \mathrm{C}$ in a circulating water bath for between 2-3 hours. After incubation, the samples were transferred to QiagenMaXtract tubes (Qiagen, Crawley, UK) and the DNA extracted in $1 \mathrm{~mL}$ Phenol/Chloroform mix (SigmaAldrich Company Ltd, Poole, UK) before centrifugation (5 minutes, 3,100 rpm). The supernatant was removed and an additional $1 \mathrm{~mL}$ of Chloroform was added followed again by centrifugation ( 5 minutes, 3,100 rpm). The DNA was transferred into a new 15 $\mathrm{mL}$ tube (Corning Incorporated, USA) containing $2-3$ volumes of $100 \%$ ethanol and 250 $\mu \mathrm{L}, 10 \% 3 \mathrm{M} \mathrm{NaAc}(\mathrm{pH}$ 5.2). The DNA was then transferred again to an Eppendorf tube containing $500 \mu \mathrm{L} 80 \%$ ethanol and centrifuged ( 30 seconds, 13,200 rpm). The $80 \%$ ethanol was removed and the DNA left to air-dry for approximately 15 minutes in the 
laminar flow hood. Finally, the DNA was re-suspended in $50 \mu \mathrm{L}$ SM-PCR clean ultrapure $\mathrm{H}_{2} \mathrm{O}$ (ultra-pure water that has been UV irradiated).

\subsection{DNA Digestion and Precipitation}

Approximately $5 \mu \mathrm{g}$ of each DNA sample was digested with 1x NEB buffer 2, $20 \mathrm{ng}$ Bovine Serum Albumin (BSA) and $25 \mathrm{U}$ Msel restriction enzyme (New England Biolabs (NEB), Hitchin, UK) for 2 hours at $37^{\circ} \mathrm{C}$ in a circulating water bath. Msel cleaves outside the ESTR array and distal to the PCR primer sites used for PCR amplification, therefore it was used to render genomic DNA fully soluble prior to dilution. DNA was precipitated in $40 \mu \mathrm{L}, 100 \%$ ethanol and 10\%, $3 \mathrm{M} \mathrm{NaAc}(\mathrm{pH} 5.2)$ and frozen (>2 hours, $\left.80^{\circ} \mathrm{C}\right)$. The tubes were centrifuged (20 minutes, $13,200 \mathrm{rpm}$ ) before being rotated $180^{\circ}$ and centrifuged again (20 minutes, 13,200 rpm). The supernatant was removed and the DNA pellet washed with $50 \mu \mathrm{L}, 80 \%$ ethanol and centrifuged ( 30 seconds, 13,200 rpm). The supernatant was removed and the pellet air dried for 15 minutes. The pellet was then re-suspended in $50 \mu \mathrm{L}$ SM-PCR clean ultra-pure water. The yield of digested DNA was measured.

\subsection{DNA Quantification}

The digested DNA concentration was estimated using an ND-1000 Spectrophotometer (NanoDrop Technologies, Ringmer, UK). When possible the DNA samples were diluted in a separate Eppendorf tube to a concentration of approximately $10 \mathrm{ng} / \mu \mathrm{L}$ using SMPCR clean ultra-pure water.

\subsection{Single-Molecule PCR Optimisation}

The $10 \mathrm{ng} / \mu \mathrm{L}$ DNA stock was diluted in order to establish a concentration whereby one haploid genome and thus one amplifiable ESTR molecule was present in each reaction. To achieve this, the stock DNA was diluted with dilution buffer (DB) $(5 \mathrm{mM}$ Tris- $\mathrm{HCl}$ (pH 7.5), $5 \mu \mathrm{g} / \mathrm{mL}$ Salmon sperm DNA) to a variety of concentrations (Table 10). Eight reactions, each containing $1 \mu \mathrm{L}$ diluted DNA were established for their respective concentrations. They were then all amplified (PCR), blotted and an autoradiograph image obtained. 
Table 10. SM-PCR optimisation

\begin{tabular}{|l|l|}
\hline DNA Concentration & Volume DNA (stock) : Volume DB $(\boldsymbol{\mu l})$ \\
\hline $500 \mathrm{pg}$ & $1(10 \mathrm{ng}): 19$ \\
\hline $100 \mathrm{pg}$ & $2(500 \mathrm{pg}): 8$ \\
\hline $50 \mathrm{pg}$ & $1(500 \mathrm{pg}): 9$ \\
\hline $20 \mathrm{pg}$ & $2(500 \mathrm{pg}): 48$ \\
\hline $10 \mathrm{pg}$ & $10(20 \mathrm{pg}): 10$ \\
\hline $5 \mathrm{pg}$ & $5(20 \mathrm{pg}): 5$ \\
\hline $2 \mathrm{pg}$ & $1(20 \mathrm{pg}): 9$ \\
\hline
\end{tabular}

The DNA concentrations for SM-PCR optimisation and the volumes of DNA and dilution buffer (DB) used.

\subsection{Poisson Analysis at the Single Molecule Level}

Using the Hi Fidelity PCR system (Roche) with Betaine, the concentrations obtained from the optimisation assays were used to establish a new set of data. $10 \mu \mathrm{L}$ reactions were set up for the single-molecule concentration in 96 reactions, which were then amplified, blotted and an autoradiograph produced (Figure 11).

The amounts of amplifiable molecules were noted and their location on the PCR plate identified. In order to collate robust estimates of individual ESTR mutation frequencies in each animal, an average of approximately 150 amplifiable molecules were required to be obtained and analysed for each tissues for each individual mouse (Barber et al., 2006, Glen et al., 2008, Glen \& Dubrova, 2012). Therefore, DNA amplification was performed until a sufficient number of Ms6-hm molecules were ascertained.

\subsection{Mutation Scoring}

Positive PCR products were resolved on $40 \times 20 \mathrm{~cm}$ agarose gels. Longer gels were used to ensure the progenitor alleles separated sufficiently thereby providing an appropriate resolution in order to detect the Ms6-hm mutants.

\subsection{Statistical Analysis}

ESTR mutation frequencies, 95\% confidence intervals and standard errors were estimated from the Poisson distribution using a modified version of an approach previously described (Zheng et al., 2000a). To assist data analysis, a database was compiled for each animal representing the individual exposure parameters using Microsoft Excel 2010. Adobe Photoshop CS4 was employed in the preparation of figures included in this thesis. Statistical analysis was performed using software written 
using Microsoft Basic by Professor Y.E Dubrova alongside programs STATISTICA and SYSTAT.

\subsection{Ms6-hm Amplification and Autoradiograph Preparation}

\subsubsection{Polymerase Chain Reaction}

Since single-molecule PCR involves the dilution of bulk genomic DNA and the amplification of multiple samples, each containing approximately one amplifiable ESTR molecule, the Ms6-hm ESTR locus was amplified in $10 \mu \mathrm{L}$ reactions. The Ms6-hm ESTR locus consists of a pentanucleotide repeat GGGCA (Kelly et al., 1989; Gibbs et al., 1993), therefore amplification was performed using $0.4 \mu \mathrm{M}$ flanking primers $\mathrm{HM} 1.1 \mathrm{~F}$ (5' - AGA GTT TCT AGT TGC TGT GA - 3') and HM1.1R (5' - ATG CCT TAG AAC TGA CTC TC $-3^{\prime}$ ) primers (Sigma-Aldrich, Poole, U.K.), 10x buffer (Roche Molecular Biochemicals, USA), $200 \mu \mathrm{M}$ dNTPs (Roche), 1 M Betaine, 3.4 $\mu \mathrm{L}$ SM-PCR clean ultra-pure water and

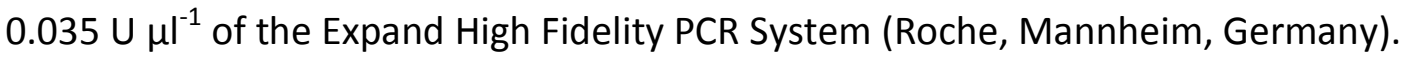
Amplification was carried out in $0.2 \mathrm{ml}$ thin-walled PCR tube strips consisting of 8 wells (Thermo Scientific, Ashford, UK). Using a PCT-225 Tetrad PCR machine (Bio-Rad, Hemel Hempstead, UK), the PCR program consisted of heated lids to denature the DNA for 3 minutes at $96^{\circ} \mathrm{C}$ before 29 cycles of, $96^{\circ} \mathrm{C}$ for 20 seconds, $58^{\circ} \mathrm{C}$ for 30 seconds and $68^{\circ} \mathrm{C}$ for 3 minutes were performed. The program terminated with a final cycle of $68^{\circ} \mathrm{C}$ for 10 minutes.

\subsubsection{Short Agarose Gel Electrophoresis}

This type of gel electrophoresis was used in the separation of DNA products for the SM-optimisation and Poisson analysis. 1\% agarose gels, $25 \times 24 \mathrm{~cm}$ in size were produced through the addition of $3 \mathrm{~g}$ of Seakem Le Agarose (Lonza, Rockland, Maine, USA) and $300 \mathrm{~mL}$ 0.5x TBE buffer (22.5 mM Tris-borate ( $\mathrm{pH}$ 8.3) and 0.5 mM EDTA). Each gel consisted of 4 rows of 26 wells into which $5 \mu \mathrm{L}$ of DNA was loaded, alongside 2 wells of $200 \mathrm{ng}$ of $1 \mathrm{~kb}$ DNA ladder (Invitrogen, Paisley, UK) per row. The DNA samples each had $5 \mu \mathrm{L}$ loading dye (5x TAE, 12.5\% Ficoll 400, (Sigma-Aldrich Company Ltd, Poole, UK) and 0.1\% Bromophenol blue added prior to loading to enable the DNA fragments to be observed. The gels were electrophoresed in 0.5x TBE running buffer, at 200 volts for 1.25 hours; until the blue loading dye had migrated three quarters of the way down the well track. 


\subsubsection{Long Agarose Gel Electrophoresis}

This type of gel electrophoresis was used for mutation scoring analysis. Prior to electrophoresis the gel tanks require balancing to ensure that the DNA fragment does not electrophorese out of the gel and into the running buffer. 1\% agarose gels, $40 \times 20$ $\mathrm{cm}$ in size were produced through the addition of $6 \mathrm{~g}$ of Seakem Le Agarose and 600 $\mathrm{mL}$ 1x TBE buffer (44.5 mM Tris-borate $\mathrm{pH} 8.3,1 \mathrm{mM}$ EDTA and $0.5 \mu \mathrm{g} / \mathrm{mL}$ Ethidium Bromide). Each gel consisted of 1 row of 27 wells. $5 \mu \mathrm{L}$ of each positive PCR reaction was loaded alongside 1 mg, 1 kb ladder and 200 ng, 200 bp ladder (Promega, Southampton, UK), which were loaded to determine the migration distance and the size of the DNA fragments, respectively. Gel electrophoresis occurs in 1x TBE buffer running buffer at 200 volts for approximately 24 hours; until the $1.6 \mathrm{~kb}$ band of the $1 \mathrm{~kb}$ ladder had migrated off the bottom of the gel.

\subsubsection{Southern Blot}

Gels were removed from their running tanks and inverted into plastic trays. They were rinsed with depurinating solution $(0.25 \mathrm{M} \mathrm{HCl}, 2 \times 5$ minutes), denaturing solution $(0.5 \mathrm{M} \mathrm{NaOH}$ and $1 \mathrm{M} \mathrm{NaCl}, 2 \times 10$ minutes) and finally in neutralising solution $(0.5 \mathrm{M}$ Tris and $3 \mathrm{M} \mathrm{NaCl}, 2 \times 5$ minutes). DNA was transferred to a MAGNA nylon transfer membrane (Gilson Scientific Ltd, Luton, UK) by capillary transfer method (Southern, 1975). The MAGNA nylon transfer membrane had been soaked in $2 x$ SSC $(0.3 \mathrm{M} \mathrm{NaCl}$ and 0.03M Na-citrate) prior to transfer and 20x SSC was used as the transfer buffer while 3MM Whatmann filter paper (Munktell, Falun, Sweden) was used to facilitate uptake of DNA to the membrane. After a minimum of 5 hours the nylon membrane was removed and rinsed in $2 \times \mathrm{SSC}$. The membrane was dried $\left(10\right.$ minutes, $\left.80^{\circ} \mathrm{C}\right)$ before the DNA was covalently cross-linked to the membrane using a UV crosslinker (Hoefer) at $7 \times 10^{4} \mathrm{~J} / \mathrm{cm}^{2}$.

\subsubsection{Probe Labelling}

Double stranded Ms6-hm probe DNA was produced via PCR using the primers HMA (5' - GGGCA GGGCA GGGCA GGGCA GG - 3') and HMB (5' - TGCCC TGCCC TGCCC TGCCC $\left.3^{\prime}\right)$, (Sigma-Aldrich, Poole, U.K.). The Ms6-hm probe (20 ng), the $1 \mathrm{~kb}(40 \mathrm{ng}$ ) and the $200 \mathrm{bp}$ (40 ng) ladder probes were all labelled with $1.5 \mu \mathrm{L} \alpha-{ }^{32} \mathrm{P}-\mathrm{dCTP}$ (PerkinElmer, Austria), for both the short and long agarose gel membranes. 
Ms6-hm DNA (20 ng) was added to $\mathrm{H}_{2} \mathrm{O}$ to make a volume of $30 \mu \mathrm{L}$. The tubes were boiled for 6 minutes to denature the DNA. Then $12 \mu \mathrm{g} \mathrm{BSA}, 6 \mu \mathrm{L}$ Oligo-labelling buffer, 8.16 U Klenow DNA polymerase (United States Biochemical Corp (USB), Cleveland, USA) and $1.5 \mu \mathrm{L}$ of $\alpha-{ }^{32} \mathrm{P}-\mathrm{dCTP}$ were added. The tubes were incubated for a minimum of 5 hours at room temperature (RT).

\subsubsection{Probe Recovery}

Incubation was stopped by adding $70 \mu \mathrm{L}$ Oligo-stop solution ( $5 \mathrm{M} \mathrm{NaCl}, 1 \mathrm{M}$ Tris (pH 7.5), 0.5M EDTA, $10 \%$ SDS, $10 \mathrm{mM}$ dCTP, $\mathrm{H}_{2} \mathrm{O}$ ). The labelled DNA was then precipitated with $90 \mu \mathrm{g}$ salmon sperm DNA, $30 \mu \mathrm{L}, 2 \mathrm{M} \mathrm{NaAc}(\mathrm{pH}$ 5.6) and $425 \mu \mathrm{L}, 100 \%$ ethanol. The solution was centrifuged ( 3 minutes, 13,200 rpm) and the supernatant discarded into a beaker of detergent. The pellet was washed with $80 \%$ ethanol and centrifuged (1 minute, 13,200 rpm) before the supernatant was again removed into a beaker of detergent. The pellet was re-suspended in $600 \mu \mathrm{L}$ ultra-pure water. The appropriate labelled ladders, ( $30 \mu \mathrm{L}, 1 \mathrm{~kb}$ for short gel, $30 \mu \mathrm{L}$, both $1 \mathrm{~kb}$ and $200 \mathrm{bp}$ for long gels) were added to the labelled Ms6-hm probe which was boiled ( 6 minutes, $100^{\circ} \mathrm{C}$ ) to denature the DNA prior to hybridisation. The probes were finally added to $\sim 10 \mathrm{~mL}$ Church buffer (0.5 M Na-Phosphate, 7\% SDS and 1 mM EDTA (Fisher Scientific, Loughborough, UK) and then to the hybridisation bottles containing the membranes.

\subsubsection{Hybridisation}

The DNA-bound MAGNA nylon transfer membranes were rolled between two nylon meshes and placed into the hybridisation bottles along with 15 $\mathrm{mL}$ Church buffer(0.5M Na Phosphate, 7\% SDS, 1 mM EDTA). The membranes were then prehybridised $\left(20\right.$ minutes, $65^{\circ} \mathrm{C}$ ) in a Mini 10 hybridisation oven (Thermo Scientific, Ashford, UK).

Upon recovery of the probes (Probe Recovery section) the Church buffer was removed and the labelled DNA probes were added to $10 \mathrm{~mL}$ Church buffer and then to the hybridisation bottles containing the membranes. The probes were then left to hybridise to the DNA-bound membranes $\left(>5\right.$ hours, $65^{\circ} \mathrm{C}$ ) in the Mini 10 hybridisation oven. 
Once the labelled probes had hybridised to the DNA-bound nylon membrane the unbound probes were removed through washing. Phosphate wash solution (0.04M NaPhosphate, $0.5 \%$ SDS) was added to the hybridisation bottle firstly for 1 minute (RT) before being removed and secondly for 10 minutes $\left(65^{\circ} \mathrm{C}\right)$. High stringency wash solution $(0.1 \times \mathrm{SSC}$ and $0.01 \% \mathrm{SDS})$ was then used for further 10 minute washes $\left(65^{\circ} \mathrm{C}\right)$ until the radioactivity levels were $\sim 10$ counts per second (cps), (Mini 900 Ratemeter, Thermo Scientific, Ashford, UK).

\subsubsection{Autoradiography}

The membranes were then removed from the hybridisation bottles and washed $(\sim 500$ $\mathrm{mL}, 2 \times \mathrm{SSC}$ ) and covered in Saran wrap. The membrane was taped, DNA side up, into an autoradiograph cassette (Genetic Research Instrumentation GRI, Braintree, UK) containing an intensifying screen and a Fuji Rx100 X-ray film inserted. The radioactivity level of the membrane determined the length of time for which the cassette was stored at $-80^{\circ} \mathrm{C},(>50 \mathrm{cps}=2-3$ hours or $<50 \mathrm{cps}=$ overnight $)$.

The films were developed using manual dip tanks instead of a developing machine in order to maintain control over the amount of film development. The cassettes were removed from the $-80^{\circ} \mathrm{C}$ freezer and placed into a drying oven for 10 minutes. Films were removed from the cassette and initially placed into the Developing solution (RGX-ray developer, Champion Photochemistry, Malaysia) before the developing reaction was terminated by the Stop solution (13.5 litres $\mathrm{H}_{2} \mathrm{O}, 135 \mathrm{~mL}$ Acetic acid (Acros Organics, Geel, Belgium) and finally the image was fixed onto the film by placing it into the Fixer solution (RG-X-ray fixer, Champion Photochemistry, Malaysia) until the film appeared clear.

\subsubsection{Mutation Scoring and Sizing of ESTR Mutants}

All de novo ESTR mutants pertaining to each tissue, exposure dose and time point, along with the age-matched sham-treated controls and X-ray irradiated males were reliably scored twice through two independent markers. Mutants were scored by eye as novel DNA fragments demonstrating a shift of at least $1 \mathrm{~mm}$ relative to the progenitor allele. Smaller length-changes however were not scored as they could not be reliably scored consistently. The parental and mutant allele sizes were estimated in 
accordance with the method employed by Southern (1979) with the 200 bp DNA step ladder (Promega, UK) used as a reference.

\subsection{Microarray Analysis}

\subsubsection{RNA Extraction}

Two kidneys per animal ( $5 x$ biological replicates per group), of the indicated treatments were collected and fast frozen in liquid nitrogen before being stored at $80^{\circ} \mathrm{C}$.

In all instances, RNA samples were extracted individually from two kidneys taken from each animal and pooled. RNA extractions were performed using a modified protocol of the TRIzol reagent (SIGMA-ALDRICH, St Louis, USA) method, whereby the TRIzol reagent is a mixture of guanidine thiocyanate and phenol in a monophase solution which dissolves DNA, RNA and protein upon homogenisation or lysis of tissue (Chomczynski \& Sacchi, 1987, 2006).

Prior to homogenisation of tissues, the working area and Diethylpyrocarbonate (DEPC)-treated sterilised homogeniser probe were cleaned with RNaseZap (Ambion, Life Technologies Ltd, Paisley, UK) to ensure RNase-free working conditions. The homogeniser probe was assembled into the Multi-Gen 7 homogenizer (PRO Scientific Inc., Oxford, CT, USA) and cooled in dry ice for 30-40 seconds prior to use. The kidneys were retrieved onto dry ice and transferred individually into a sterile $15 \mathrm{~mL}$ centrifuge tube containing $7 \mathrm{~mL}$ TRIzol reagent, (10-100 mg of tissue per $\mathrm{mL}$ TRIzol reagent). Upon addition, the kidney was homogenised for 20 seconds and left for 5 minutes at room temperature. After incubation, $1.4 \mathrm{~mL}$ 1-bromo-3-chloro-propane (Sigma B9673), (200 $\mu \mathrm{L}$ per $\mathrm{mL}$ TRIzol reagent), was added to lessen genomic DNA contamination. The TRIzol lysate was then vigorously mixed on the vortex at full speed for 30 seconds, and left for a further 3 minutes at room temperature. Afterwards, the contents of the tube were aliquoted into 8 labelled $1.5 \mathrm{~mL}$ RNase-free Eppendorf tubes and phaseseparated through centrifugation (10 minutes, 12000 relative centrifugal force (rcf), $4^{\circ} \mathrm{C}$, cooled at $4^{\circ} \mathrm{C}$ before use). The RNA containing upper aqueous phase was transferred to a $1.5 \mathrm{~mL}$ RNase-free Eppendorf tube containing $560 \mu \mathrm{L}$ isopropanol (Sigma), (1 $\mu \mathrm{L}$ per $1 \mu \mathrm{L}$ aqueous phase), while the DNA and protein containing phases; 
interphase and the organic phase were discarded. The RNA isolates were inverted 20 times to mix and left at room temperature for 10 minutes. They were then centrifuged (10 minutes, $12000 \mathrm{rcf}, 4^{\circ} \mathrm{C}$ ) to form an RNA pellet. The samples were collected on ice, the supernatant discarded and the RNA pellet washed with $1 \mathrm{~mL},\left(-20^{\circ} \mathrm{C}\right), 75 \%$ RNasefree ethanol (3:1 ethanol/DEPC water). Samples were again centrifuged (5 minutes, $12000 \mathrm{rcf}, 4^{\circ} \mathrm{C}$ ) and the RNA pellet was again collected on ice before the ethanol was discarded and the wash repeated. After centrifugation (5 minutes, $12000 \mathrm{rcf}, 4^{\circ} \mathrm{C}$ ), the samples were left in the centrifuge $\left(4^{\circ} \mathrm{C}\right)$ for a further 5 minutes before collection, again on ice. The ethanol was removed completely and the RNA pellet air dried for 5 minutes on ice. The RNA pellets were re-suspended in 80-150 $\mu \mathrm{L}$ nuclease-free water (1/1000 final, DEPC/ $\left.\mathrm{H}_{2} \mathrm{O}\right)$, depending on their size. Upon complete re-suspension the RNA sample was temporarily pooled into a single solution to measure the concentration and integrity. Afterwards, each RNA sample was stored at $-80^{\circ} \mathrm{C}$.

\subsection{RNA Quantification}

After extraction, $5 \mu \mathrm{L}$ of each RNA sample was transferred to a new Eppendorf tube and the total RNA concentration quantified using an ND-1000 Spectrophotometer (Thermo Fisher Scientific, USA).

\subsubsection{RNA Integrity Measurement}

The integrity of the RNA samples was assessed on an Agilent 2100 Bioanalyzer (Agilent Technologies, Wokingham, UK) using an Agilent RNA 6000 Nano Kit (Agilent Technologies, Inc. Waldbronn, Germany). Each Agilent RNA 6000 Nano Kit contains multiple RNA Nano Chips, each of which is equipped with an interconnected set of micro-channels that is used for size-dependant separation of the nucleic acid fragments as they are driven through it electrophoretically. Each RNA sample's integrity is evaluated through the production of a RNA Integrity Number (RIN). The RIN index provides a quantitative value ranging from 1 to 10 that facilitates the assessment of the RNA integrity through classification of the eukaryotic total RNA (Schroeder et al., 2006). The resulting electropherogram exhibits at least two distinct peaks representing the $18 \mathrm{~S}$ and $28 \mathrm{~S}$ ribosomal RNA (Figure 10).

Prior to evaluation, all Agilent RNA 6000 Nano Reagents equilibrated to room temperature for 30 minutes, while each RNA sample was diluted to $250 \mathrm{ng} / \mu \mathrm{L}$ using 
RNase-free water. The RNA ladder had been previously heat denatured $\left(70^{\circ} \mathrm{C}, 2\right.$ minutes) and stored at $-80^{\circ} \mathrm{C}$.

Prior to the preparation of the gel matrix, the RNA 6000 Nano gel matrix, required filtering, $550 \mu \mathrm{L}$ of was pipetted into a spin filter and centrifuged (1500 rcf, 10 minutes, room temperature). Thereafter, $65 \mu \mathrm{L}$ of the filtered gel was aliquoted into a $1.5 \mathrm{~mL}$ RNase-free Eppendorf tube whilst the remaining filtered gel was stored at $4{ }^{\circ} \mathrm{C}$ for up to 4 weeks. The RNA 6000 Nano dye concentrate was then vortexed for 10 seconds and centrifuged before $1 \mu \mathrm{L}$ was added to the $65 \mu \mathrm{L}$ filtered gel. The subsequent gel-dye solution was mixed on a vortex and centrifuged (13000 rcf, 10 minutes, room temperature) and stored at room temperature while a new RNA 6000 Nano chip was added to the chip priming station. $9 \mu \mathrm{L}$ of the gel-dye mix was added to the Nano chip into the well-marked $(\mathbf{G})$. The chip priming station was then closed and the syringe plunger pressed down for exactly 30 seconds before being released. Five seconds after release the plunger was replaced to the starting position and the chip priming station opened once more. A further $9 \mu \mathrm{L}$ of the gel-dye mix was then added into the two wells marked $(\mathbf{G})$ of the Nano chip and the remaining gel-dye solution discarded. Next, $5 \mu \mathrm{L}$ of RNA 6000 Nano maker was pipetted into all 12 sample wells in addition to the ladder well. RNA samples were denatured $\left(70^{\circ} \mathrm{C}, 2\right.$ minutes) immediately prior to loading, where $1 \mu \mathrm{L}$ of denatured RNA sample was added to each of the 12 sample wells and $1 \mu \mathrm{L}$ of the prepared ladder was pipetted into the ladder well. If any wells remained unused a further $1 \mu \mathrm{L}$ of the Nano Marker was added. Once all the wells had been loaded, the chip was removed from the priming station and placed in the adapter of the IKA vortexer. The chip was then vortexed for 1 minute at $2400 \mathrm{rpm}$ and placed into the Agilent 2100 Bioanalyzer for scanning. 


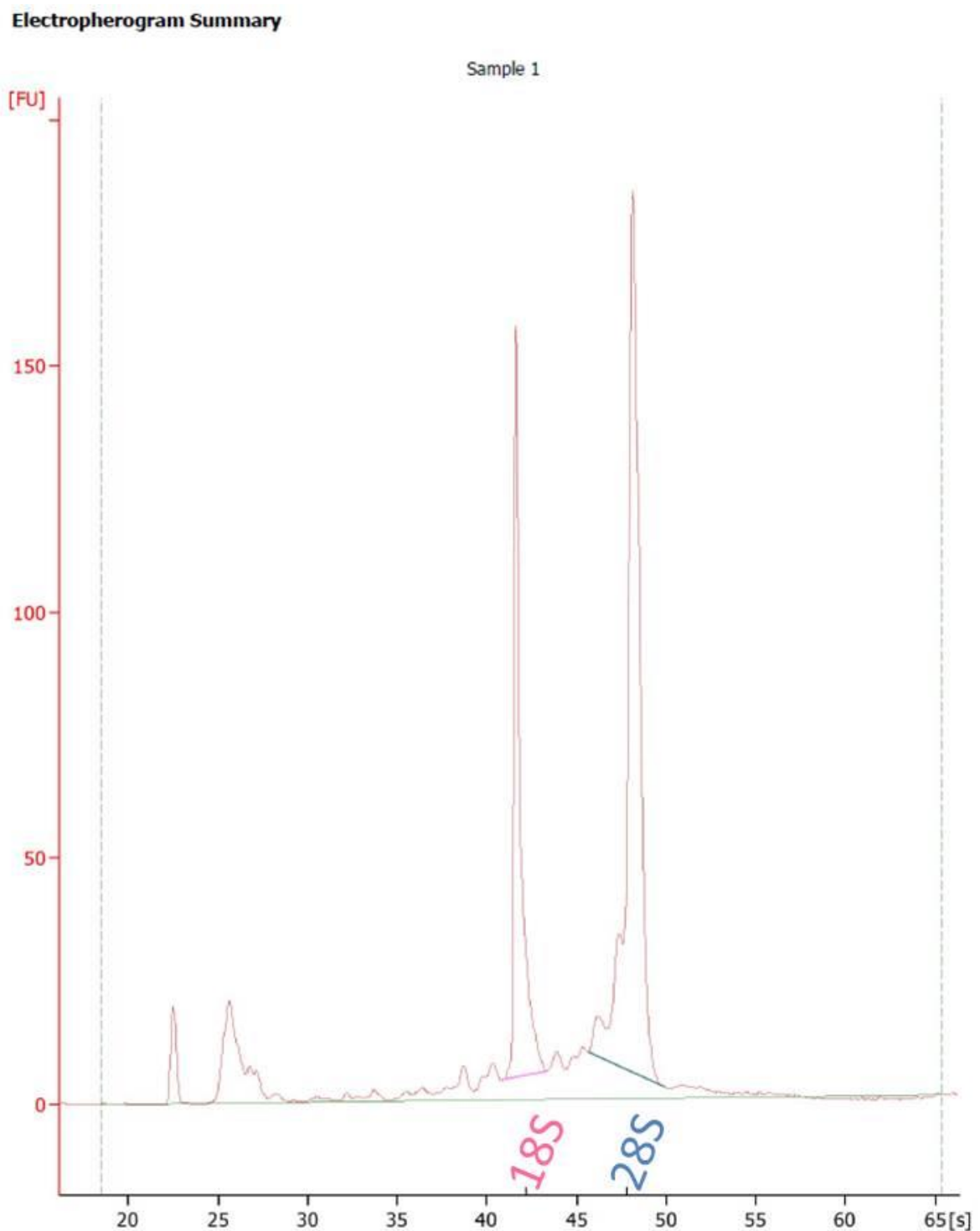

Figure 10. RNA Electropherogram. An electropherogram illustrating the total RNA peaks of the successful sample X-1.10. The RIN index for the sample shown is 9.7. Characteristic regions for ribosomal peaks and the lower marker are displayed. 


\subsection{One-Colour Microarray-based Gene Expression Analysis}

Through the use of T7 RNA Polymerase Blend and Cyanine 3-CTP Dye Pack (Agilent Technologies Inc, TX 78612, USA), the Agilent Low Input QuickAmp Labelling Kit (Agilent Technologies Inc, TX 78612, USA) generates fluorescently labelled cRNA. The gene expression in experimental and control samples was measured through the hybridisation of the fluorescent cRNA onto Agilent SurePrint G3 format 8x60k microarray slides (Agilent Technologies Inc, TX 78612, USA). One-color arrays result in absolute fluorescence intensities, which are assumed to be monotonically (if not linearly) related to the abundance of mRNA species complementary to the probes on the array.

\subsubsection{Template total RNA with Spike-In}

After extraction, each sample was diluted to a total RNA concentration $40 \mathrm{ng} / \mu \mathrm{L}$ and $1.5 \mu \mathrm{L}$ transferred to a new $1.5 \mathrm{~mL}$ RNase-free microcentrifuge tube, on ice, ready for amplification and labelling.

Before use the RNA Spike-In One Colour Mix (Agilent Technologies Inc, TX 78612, USA) was vigorously mixed on a vortex mixer and heated in a circulating water bath $\left(37^{\circ} \mathrm{C}, 5\right.$ minutes). Upon removal the RNA Spike Mix was once again vigorously mixed on a vortex mixer and briefly centrifuged ready to prepare the serial dilutions for Cyanine 3labelling. The RNA Spike Mix was serial diluted in $1.5 \mathrm{~mL}$ RNase-free microcentrifuge tubes as per the manufacturer's instructions, $\left(1^{\text {st }} 1: 20,2^{\text {nd }} 1: 25,3^{\text {rd }} 1: 20,4^{\text {th }} 1: 4\right)$, whereby the fourth dilution is at a 40000 -fold final dilution. $2 \mu \mathrm{L}$ of the fourth dilution was added to a new $1.5 \mathrm{~mL}$ RNase-free microcentrifuge tube containing $1.5 \mu \mathrm{L}$ total RNA, resulting in a total volume of $3.5 \mu \mathrm{L}$ and $25 \mathrm{ng}$ total RNA. The first dilution of the spike mix was stored for up to 2 months at $-80^{\circ} \mathrm{C}$ while the others were discarded.

\subsubsection{Cyanine 3-Labelling}

$0.8 \mu \mathrm{LT7}$ Primer Mix was added to $1 \mu \mathrm{L}$ nuclease-free water and the resulting $1.8 \mu \mathrm{L}$ solution aliquoted into the tube containing the total RNA and RNA spike-in forming a total volume of $5.3 \mu \mathrm{L}$. The primer and template mix was denatured in a circulating water bath $\left(65^{\circ} \mathrm{C}, 10\right.$ minutes), collected on ice and incubated for 5 minutes. While the primer-template mix was incubating on ice, the $5 x$ First Strand Buffer was pre-warmed in a heat block $\left(80^{\circ} \mathrm{C}, 4\right.$ minutes) to ensure adequate re-suspensions of the buffer 
components. Afterwards, the 5x First Strand Buffer was briefly mixed on the vortex and centrifuged. Upon re-suspension, the 5x First Strand Buffer was added along with the other components in Table 11 to form the cDNA master mix.

Table 11. cDNA master mix

\begin{tabular}{|l|l|}
\hline Components & Volume $(\mu \mathrm{L})$ per reaction \\
\hline $5 x$ First Strand Buffer & 2 \\
\hline 0.1 M DTT & 1 \\
\hline 10 mM dNTP Mix & 0.5 \\
\hline Affinity Script RNase Block Mix & 1.2 \\
\hline Total Volume & 4.7 \\
\hline
\end{tabular}

The components and required volume of each required in the cDNA master mix for each RNA sample.

4.7 $\mu \mathrm{L}$ of cDNA master mix was added to each sample tube, bringing the total volume to $10 \mu \mathrm{L}$, and mixed. The samples were then incubated in a circulating water bath $\left(40^{\circ} \mathrm{C}\right)$ for two hours. After the incubation period, the samples were moved to another circulating water bath at $70^{\circ} \mathrm{C}$ for a further 15 minutes and finally incubated on ice for 5 minutes. While the samples were incubating on ice, the Transcription Master Mix was prepared (Table 12).

Table 12. Transcription master mix

\begin{tabular}{|l|l|}
\hline Components & Volume $(\mu \mathrm{L})$ per reaction \\
\hline Nuclease-free water & 0.75 \\
\hline 5x Transcription Buffer & 3.2 \\
\hline 0.1 M DTT & 0.6 \\
\hline NTP Mix & 1 \\
\hline T7 RNA Polymerase Blend & 0.21 \\
\hline Cyanine 3-CTP & 0.24 \\
\hline Total Volume & 6 \\
\hline
\end{tabular}

The components and required volume of each required in the Transcription master mix for each RNA sample.

$6 \mu \mathrm{L}$ of transcription master mix was added to each sample tube resulting in a total volume of $16 \mu \mathrm{L}$ for each reaction. The samples were mixed and once again incubated in a circulating water bath $\left(40^{\circ} \mathrm{C}\right)$ for two hours. Following incubation, the newly amplified, cyanine 3-labelled was purified using Qiagen RNeasy Mini Kit (Qiagen Inc., Valencia CA) and the manufacturer's recommended protocol. 


\subsubsection{Cy3-RNA Clean-up}

Prior to the first use of Buffer RPE 4 volumes ( $44 \mathrm{~mL}$ ) 100\% ethanol was added to produce a working solution.

Upon removal from the water bath, on ice, $84 \mu \mathrm{L}$ RNase-free water was added to each cRNA sample ensuring a total starting volume of $100 \mu \mathrm{L}$. Afterwards, $350 \mu \mathrm{L}$ Buffer RLT was added and the solutions well mixed before $250 \mu \mathrm{L} 100 \%$ ethanol was added to the diluted RNA and mixed by pipetting the solution up and down. The sample was then transferred to an RNeasy Mini spin column which had been supplied with a $2 \mathrm{~mL}$ collection tube attached and centrifuged ( 30 seconds, $13 \mathrm{rcf}, 4^{\circ} \mathrm{C}$ ). The flow-through was discarded along with the collection tube, and the spin column replaced back into a new $2 \mathrm{~mL}$ collection tube before $500 \mu \mathrm{L}$ Buffer RPE was added to the spin column. The samples were again centrifuged ( 30 seconds, $13 \mathrm{rcf}, 4^{\circ} \mathrm{C}$ ) and the flow-through once more discarded, however the spin column was replaced back into the same collection tube before a further $500 \mu \mathrm{L}$ Buffer RPE was added to the column. Samples were again centrifuged ( 1 minute, $13 \mathrm{rcf}, 4^{\circ} \mathrm{C}$ ) and the flow-through and collection tube again discarded. The spin column was transferred to a new collection and spun again (30 seconds, $13 \mathrm{rcf}, 4^{\circ} \mathrm{C}$ ) in order to remove any Buffer RPE potentially left on or near the rim of the column alongside any left on the outside of the column. Again the collection tube was discarded and the spin column placed into a fresh $1.5 \mathrm{~mL}$ collection tube. The purified cRNA samples were eluted through the addition of $30 \mu \mathrm{L}$ RNase-free water directly onto the RNeasy filter membrane. The samples were left for 1 minute on ice before centrifuged ( 30 seconds, $13 \mathrm{rcf}, 4^{\circ} \mathrm{C}$ ). The RNeasy spin column was discarded and the cRNA sample placed on ice ready for quantification.

\subsection{Quantifying cRNA}

Dye incorporation and CRNA yield for all cRNA samples were quantified using the NanoDrop ND-1000 UV-VIS Spectrophotometer.

Again, $5 \mu \mathrm{L}$ of each cRNA sample was removed into a new sterile RNase-free Eppendorf tube and the NanoDrop software initialised. The microarray measurement tab was selected and the RNA-40 sub-tab was selected as the sample type. The instrument was initially blanked with $1.5 \mu \mathrm{L}$ of the same nuclease-free water used to elute the cRNA samples. Thereafter, $1.5 \mu \mathrm{L}$ of each sample was loaded on the instrument sample 
loading area and the following categories recorded; Cyanine 3 dye concentration (pmol/ $\mu \mathrm{L})$, RNA absorbance ratio (260 nm/280 nm) and cRNA concentration (ng/ $\mu \mathrm{L})$.

Through the use of these three categories and Equations 3 and 4, the cRNA yield ( $\mu \mathrm{g}$ ) and specific activity of each sample was determined.

\section{Equation 3}

$$
\mu \mathrm{g} \text { of } \mathrm{cRNA}=\frac{\text { Concentration of cRNA } \times 30 \mu \mathrm{L} \text { (elution volume) }}{1000}
$$

\section{Equation 4}

$$
\text { pmol Cy3 per } \mu \mathrm{g} \text { cRNA }=\frac{\text { Concentration of } \mathrm{Cy} 3}{\text { Concentration of cRNA }} \times 1000
$$

The Agilent SurePrint G3 format 8x60k slides are formatted with 8 sub-arrays printed on each 1-inch $\times 3$-inch glass slide. The minimum cRNA yield $(\mu \mathrm{g})$ loaded onto each slide was 0.825 while the minimum specific activity (pmol Cy3 per $\mu \mathrm{g}$ cRNA) was 6 . Any samples that did not meet these requirements were not loaded onto the slide.

\subsection{Hybridisation}

Hybridisation was performed using the Agilent Gene Expression Hybridisation Kit (Agilent Technologies Inc, TX 78612, USA) according to the manufacturer's protocol.

Firstly, $500 \mu \mathrm{L}$, nuclease-free water was pipetted into the supplied vial containing lyophilized 10x Gene Expression Blocking Agent and gently mixed on the vortex until the pellet was re-suspended. Failure to re-suspend the pellet resulted in the mixture being heated for 5 minutes at $37^{\circ} \mathrm{C}$ to facilitate re-suspension while the addition of the Agilent-CGHBlock blocking buffer was required to minimize background signal on the array. Using the components of the Agilent High-RPM Gene Expression Hybridisation Kit (Table 13), the following master mix was prepared to hybridise $600 \mathrm{ng}$ of Cy3labelled cRNA on the SurePrint G3 format 8x60k slide. 
Table 13. Fragmentation mix

\begin{tabular}{|l|l|}
\hline Components & Volume $(\mu \mathrm{L}) /$ mass per reaction \\
\hline Cyanine 3-labelled cRNA & $600 \mathrm{ng}$ \\
\hline 10x Gene Expression Blocking Agent & 5 \\
\hline Nuclease-free water & Bring volume to 24 \\
\hline $25 x$ Fragmentation Buffer & 1 \\
\hline Total Volume & 25 \\
\hline
\end{tabular}

The fragmentation mix for each sample undergoing hybridisation to a SurePrint G3 format $8 \times 60 \mathrm{k}$ slide.

After addition, each sample was incubated for exactly 30 minutes in a circulating water bath $\left(60^{\circ} \mathrm{C}\right)$ in order to fragment the RNA. Immediately after 30 minutes the samples were cooled on ice for 1 minute and $25 \mu \mathrm{L} 2 \mathrm{x}$ Hi-RPM Hybridization Buffer added to terminate the fragmentation reaction. The samples were carefully mixed through pipetting up and down in an attempt to avoid the introduction of bubbles, centrifuged (13000 rcf, 1 minute, room temperature) and $40 \mu \mathrm{L}$ of each sample immediately loaded onto their respective Agilent SureHyb Gasket Slide well (Agilent Technologies Inc, TX 78612, USA). Upon completion, the SurePrint G3 microarray slide was slowly lowered on top of the SureHyb gasket slide ensuring that the slides 'active side' was facing towards the gasket slide. The SureHyb chamber cover was applied to sandwich the slides and assembly clamp secured. The assembly chamber was then placed into the rotisserie of the hybridisation oven and hybridisation was performed for 17 hours at $65^{\circ} \mathrm{C}$ rotating at $10 \mathrm{rpm}$ according to the company's recommendations.

\subsection{Washing of Hybridised Arrays}

To reduce the potential of array wash artefacts, $2 \mathrm{~mL}$ Triton X-102 (10\%) was added to the Gene Expression Wash Buffers prior to their first use. Furthermore, to ensure optimal wash conditions the Gene Expression Wash Buffer 2 was pre-warmed at $37^{\circ} \mathrm{C}$ in a circulating water bath for 17 hours.

Once hybridisation was complete, the assembly chamber was removed from the hybridization oven, disassembled and the array-gasket sandwich quickly transferred to slide-staining dish 1, where it was completely submerged in Gene Expression Wash Buffer 1 (room temperature). Keeping the microarray slide submerged with the numeric barcode facing upwards the array-gasket sandwich was carefully pried apart. Keeping the barcode at the top, the slide was transferred into a slide rack positioned in 
the slide-staining dish 2, again containing the Gene Expression Wash Buffer 1 and agitated vigorously for 1 minute. Immediately prior to the conclusion of the wash the slide-staining dish 3 was filled with the pre-warmed the Gene Expression Wash Buffer $2\left(37^{\circ} \mathrm{C}\right)$. The slide rack was then transferred to slide-staining dish 3 and again agitated vigorously for 1 minute. The slide was then slowly removed from the slide-rack minimising the potential of any wash solution remaining on the slide and transferred with the Agilent barcode facing upwards to a slide holder again for scanning.

\subsection{Array Scanning}

The slides were scanned and fluorescent signals detected using the Agilent High Resolution C Scanner (G2539A) according to the manufacturer's protocol. Raw microarray image files were created and the resulting TIFF images were processed using Agilent's Feature Extraction Software Version 10.5.1.1 (protocol: GE1_105_Dec08).

\subsection{Data Normalisation \& Quality Control}

Information from probe features was extracted from the microarray scan data thus allowing the measurement of gene expression, while the software also generated QC reports using the protocols specific for the microarray assay. The microarray data were analysed using GeneSpring software, version 12.6 (Agilent Technologies).

To enable inter-array comparisons between Agilent one-colour microarrays signals, the raw signal intensity values for each set of one-colour microarrays were log transformed $\left(\log _{2}\right)$ and globally normalised using the $75^{\text {th }}$ percentile of all non-control probes on the microarray, as specified by the manufacturer.

Statistical significance of a probes' expression was later determined using $P$ values calculated by unpaired $t$ test analysis and corrected for multiple-testing with Benjamini-Hochberg false discovery rate (FDR) of $P<0.05$. Of those probes exhibiting a differential expression of statistical significance (FDR value $<0.05$ ), only those presenting a fold increase or decrease of two or more in expression when compared to sham-treated controls were classified as differentially expressed. 


\section{Results}

\subsection{Experimental Design}

Universal exposure to extremely low-frequency magnetic fields within the developed world, in conjunction with often contradictory data, has culminated in a considerable amount of concern regarding the effects of ELF-MF on biological systems. Thus, in view of the conflicting results, it was the aim of this study to provide an in depth analysis of potential molecular changes induced by ELF-MF exposure in the germline and somatic tissues of male mice.

Mutation frequencies were evaluated in both the somatic and germline tissues of $\mathrm{BALB} / \mathrm{c} \times \mathrm{CBA} / \mathrm{Ca} \mathrm{F}_{1}$ hybrid male mice, using a highly sensitive technique that employs the use of extremely unstable expanded simple tandem repeat (ESTR) loci. The use of ESTR loci provides a highly sensitive biomarker for monitoring mutation induction, which has previously been successfully utilised in the analysis of ionising radiation (Dubrova et al., 1993; Mughal et al., 2012), chemical mutagens (Vilarino-Guell et al., 2003) and anticancer drugs (Glen et al., 2008). Germline and somatic mutation frequencies were detected at the Ms6-hm ESTR locus using single-molecule PCR (SMPCR). This approach involves the bulk dilution of genomic DNA in order to isolate and amplify approximately one ESTR molecule per reaction (Yauk et al., 2002). The utilisation of SM-PCR therefore offered several advantages to mutation screening, in providing unlimited access to de novo mutant alleles from a single individual, and the direct analysis of multiple sample equivalents from both the somatic and germline cells of a single individual. As a result SM-PCR provides a more robust estimate of individual mutation rates in both somatic and germline cells, while dramatically reducing the number of mice needed to do so.

Mutation analysis was performed in $\mathrm{BALB} / \mathrm{C} \times \mathrm{CBA} / \mathrm{Ca}_{1}$ hybrid male mice compared to males of an inbred strain in an attempt to more accurately score Ms6-hm mutants. As in previous studies (Yauk et al., 2002; Glen et al., 2008; Abouzeid Ali et al., 2012; Glen \& Dubrova, 2012; Mughal et al., 2012; Voutounou et al., 2012), de novo ESTR mutants were to be identified as novel DNA fragments demonstrating a shift of at least $1 \mathrm{~mm}$ relative to the progenitor allele. Inbred strains present Ms6-hm heterozygosity owing to the presence of two very similar-sized progenitor alleles which is problematic when 
identifying mutants presenting such minimal shifts in distance. In contrast, the use of $\mathrm{BALB} / \mathrm{c} \times \mathrm{CBA} / \mathrm{Ca} \mathrm{F}_{1}$ hybrid male mice within the current study substantially facilitated the process of ESTR mutation scoring. Such mice possess two differently-sized Ms6-hm alleles, which have been consistently and readily amplified across previous experiments (Yauk et al., 2002; Glen et al., 2008; Glen \& Dubrova, 2012; Voutounou et al., 2012). As such, $F_{1}$ hybrid male mice provided a well-spaced banding pattern, through which length-change mutations were easily scored at both the BALB/c- ( 2.5 $\mathrm{kb})$ and CBA/Ca- derived alleles ( $\sim 3.3 \mathrm{~kb}$ ), in addition to the unambiguous establishment of the mutant bands allelic origin.

Using this system, seven-week old male $\mathrm{BALB} / \mathrm{C} \times \mathrm{CBA} / \mathrm{Ca}$ hybrid mice were exposed to 10, 100 and $300 \mu$ T for 2 or 15 hours (Table 14). Blood and sperm DNA samples were extracted 12 weeks post-exposure and the frequencies at which mutations were induced at the Ms6-hm locus calculated. Tissue samples were also collected from additional age-matched sham-treated hybrid males (control group) alongside those exposed to $1 \mathrm{~Gy}$ acute X-rays (positive controls). Given the results of previous studies (Dubrova et al., 1998a; Mughal et al., 2012), such an acute exposure (1 Gy X-rays) causes a statistically significant increase in the frequency of Ms6-hm mutants. Thus, it was used as a positive control to validate the SM-PCR technique. According to the results of previous studies (Yauk et al., 2002; Barber et al., 2009; Hardwick et al., 2009), ESTR mutation induction occurs mostly, if not solely, in replication-proficient mitotic cells. The extraction of DNA samples 12 weeks post-exposure therefore ensured that the evaluated sperm was derived from exposed $A_{s}$ spermatogonia (Searle, 1974). Likewise, the extremely high-turnover of hematopoietic tissues in mice ensured the majority of nucleated blood cells were also derived from exposed replication-proficient stem cells (Metcalf, 1988).

An acute exposure of 2 - or 15 hours to a continuous $50 \mathrm{~Hz}$ magnetic field was applied due to several studies previously observing a dose-dependent increase in DNA strand breaks both in vitro and in vivo following acute (2 hour) exposure (Lai \& Singh, 1997; Ivancsits et al., 2002, 2003b; Focke et al., 2010), with the response plateauing at an exposure of 15 hours (Ivancsits et al., 2002). Similarly, the field's magnetic flux densities employed in the present study $(10,100$ or $300 \mu \mathrm{T})$ have previously illustrated 
genotoxic properties in cells exposed both in vivo and in vitro (Lai \& Singh, 1997, 2004; Ivancsits et al., 2003b). While these flux densities are comparatively high in relation to household levels associated with $\mathrm{CL}(<0.1 \mu \mathrm{T})$ and those capable of small but significant increase in DNA breakage $(0.035 \mu \mathrm{T})$ (Ivancsits et al., 2003b), these values are in accordance with the recommended International Commission on Non-Ionising Radiation reference limits associated with the general public (International Commission on Non-Ionizing Radiation Protection, 1998; International Commission on Non-Ionizing Radiation Protection, 2010). Moreover, any confirmed adverse effects even witnessed at $100 \mu \mathrm{T}$ would have profound implications for risk assessment, including the need to re-examine the exposure parameters for ELF-MFs.

Thus, using single-molecule PCR, the frequency of mutations at the Ms6-hm mouse ESTR locus was established in sperm and blood samples exposed to $50 \mathrm{~Hz}$ magnetic fields of 10, 100 or $300 \mu$ T for 2 or 15 hours, and measured against those in agematched sham-treated and irradiated (acute 1 Gy X-rays) BALB/c x CBA/Ca hybrid $F_{1}$ males.

Table 14. Experimental Design

\begin{tabular}{|l|l|l|l|}
\hline & \multicolumn{2}{|l|}{ Number Analysed } \\
\hline Group & Duration & Blood & Sperm \\
\hline Sham-treated & 2 hours & 5 & 4 \\
& 15 hours & 5 & 5 \\
\hline $10 \mu \mathrm{T}$ & 2 hours & 5 & 5 \\
& 15 hours & 5 & 5 \\
\hline $100 \mu \mathrm{T}$ & 2 hours & 5 & 5 \\
& 15 hours & 5 & 5 \\
\hline $300 \mu \mathrm{T}$ & 2 hours & 5 & 5 \\
& 15 hours & 5 & 5 \\
\hline X-ray sham-treated & - & 5 & 5 \\
\hline X-ray acute 1 Gy & - & 5 & 5 \\
\hline Total & - & 50 & 49 \\
\hline Total number of animals analysed & & \multicolumn{2}{|c|}{99} \\
\hline
\end{tabular}

The doses of ELF-MF, X-ray and sham-treatments analysed in the study, the duration for which they were exposed, the tissues analysed and the number of mice analysed for each group. 


\subsection{Optimisation}

Since de novo mutant alleles are more readily detectable as single molecules, the optimal concentration at which the amplification of, on average, a single Ms6-hm molecule occurs was determined for each individual sample. Multiple DNA stock dilutions containing concentrations of $100 \mathrm{pg} / \mu \mathrm{L}, 50 \mathrm{pg} / \mu \mathrm{L}, 20 \mathrm{pg} / \mu \mathrm{L}, 10 \mathrm{pg} / \mu \mathrm{L}, 5$ $\mathrm{pg} / \mu \mathrm{L}$ and $2 \mathrm{pg} / \mu \mathrm{L}$ were amplified and assessed by autoradiograph. The concentration at which at least one amplifiable molecule is present was identified as the concentration at which $\sim 50 \%$ of the eight PCR reactions yielded a positive result (Figure 11). However, in the instances when the optimum concentration fell between two different concentrations the median value would be implemented. For example, when a concentration of $20 \mathrm{pg} / \mu \mathrm{L}$ presented six positive reactions and $10 \mathrm{pg} / \mu \mathrm{L}$ only two, the single molecule equivalent concentration would be $\sim 15 \mathrm{pg} / \mu \mathrm{L}$.

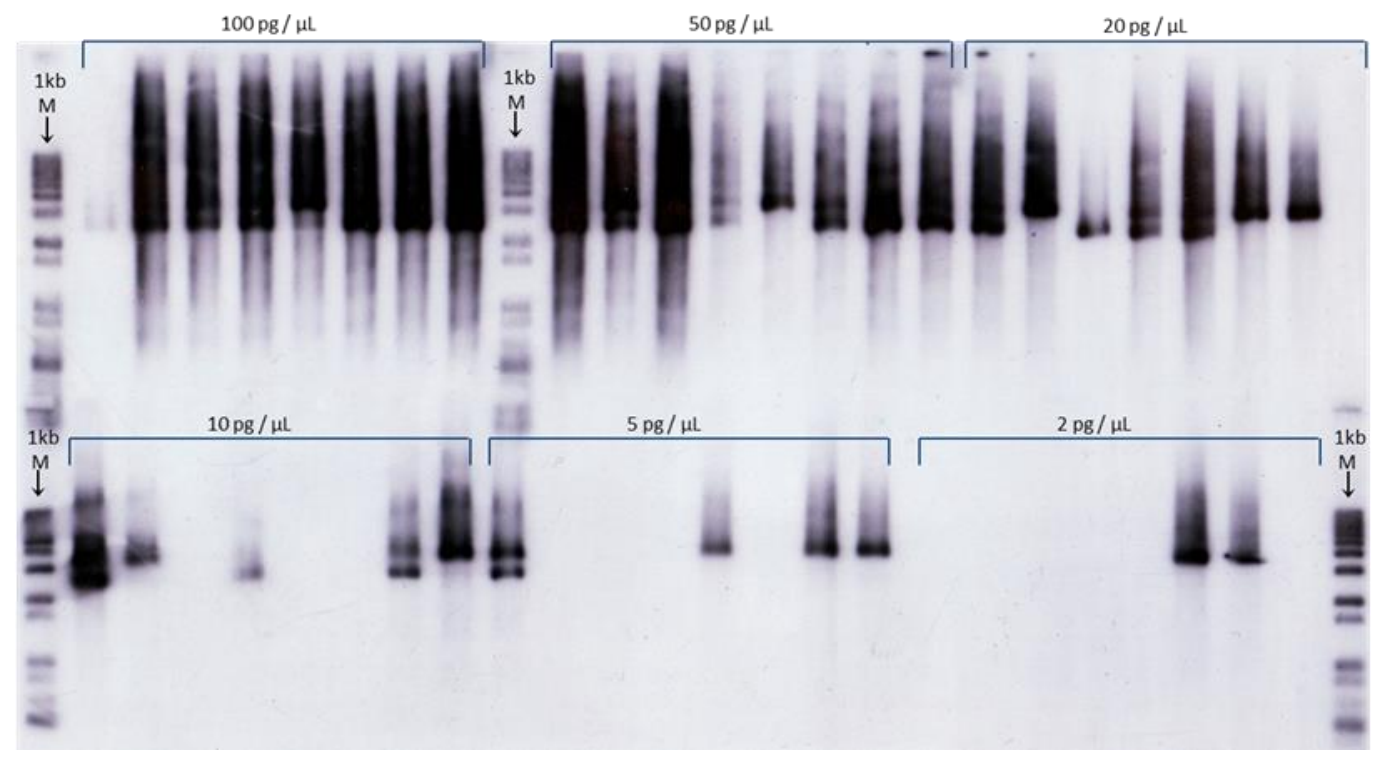

Figure 11. SM-PCR optimisation autoradiograph. Multiple dilutions on sample DNA were performed to the indicated concentrations, and subjected to Southern blot analysis. Dark bands indicated the presence of DNA. 1kb DNA step ladder (Invitrogen) was used as an identification method. A $5 \mathrm{pg} / \mu \mathrm{L}$ optimum concentration is indicated and was subsequently used for Poisson analysis.

Once the optimal concentration had been determined for each individual sample, Poisson analysis was performed in order to establish the mean number of amplifiable molecules per reaction. 


\subsection{Poisson Analysis}

DNA samples were diluted to their corresponding optimal concentrations and amplified out in 96 separate reactions (Figure 12), after which, PCR products were detected once more by Southern blot hybridisation. Poisson distribution (Equation 5, 6) was then used to calculate the mean number of amplifiable molecules per reaction. This value was subsequently multiplied by the total number of reactions to provide an estimate for the total number of amplifiable molecules per reaction.

\section{Equation 5}

$$
P=\frac{n^{-}}{k}
$$

\section{Equation 6}

$$
\lambda=-\ln (P)
$$

Where:

$P$ : Frequency of negative reactions

$\mathrm{n}^{-}$: Number of negative reactions

$k:$ Total number of reactions

$\lambda$ : Mean number of amplifiable molecules per reaction

In order to collate robust estimates of individual ESTR mutation frequencies in each animal, the process of mutation scoring requires that between 90 and 150 individual alleles be procured for each animal (Barber et al., 2006, Glen et al., 2008, Glen \& Dubrova, 2012). Therefore aliquots of each DNA sample continued to be amplified in 96 reactions and analysed until a sufficient number of positive reactions were acquired to enable mutation scoring to be accurately performed. 


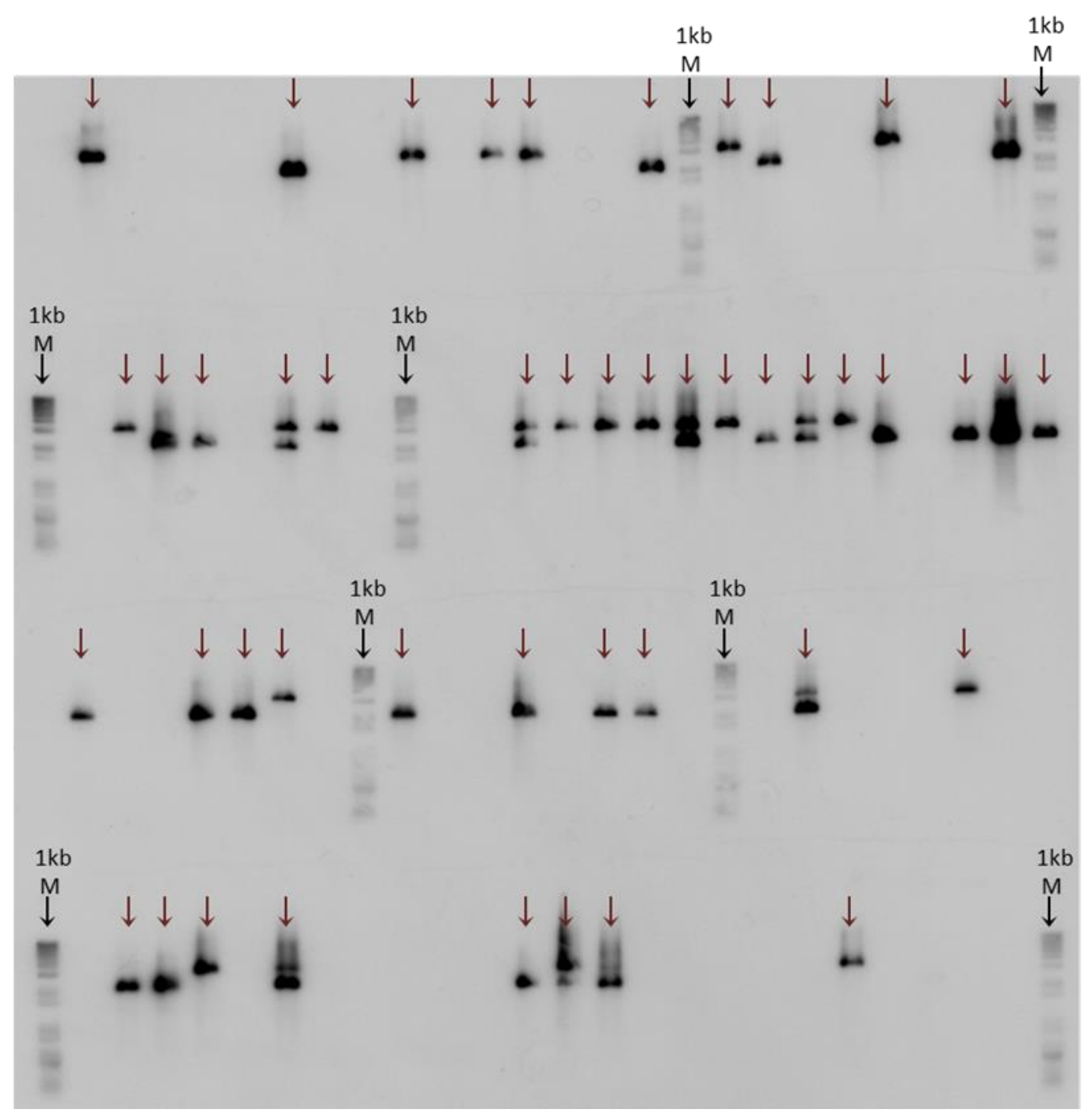

Figure 12. SM-PCR Poisson analyses. SM-PCR and Southern blot analysis of the DNA samples at the estimated single-molecule concentration. ESTR arrays in 96 reactions per plate were amplified. The single alleles were identified as positive reactions (brown arrows) and used in subsequent mutation scoring. 1 kb DNA step ladder (Invitrogen) was used as an identification method as in Figure 11. 


\subsection{Mutation Scoring}

PCR analysis was conducted on multiple samples, each containing at least one amplifiable molecule (indicated by the brown arrows, Figure 12). The ESTR mutation frequencies were determined for DNA samples of 7 week old male BALB/C $\times C B A / C a$ hybrid mice exposed to 10,100 and $300 \mu$ T for 2 or 15 hours, extracted from blood and sperm 12-weeks post-exposure.

To compare the effects of ELF-MF, tissue samples were also collected from additional age-matched sham-treated hybrid males (control group) in addition to hybrid males irradiated with an acute dose of $1 \mathrm{~Gy}$ X-rays (positive controls).

In order to provide a higher resolution for mutation scoring, PCR products were resolved on a $40 \mathrm{~cm}$-long agarose gel and detected again by Southern blot hybridisation. As mutation analysis was performed in $\mathrm{BALB} / \mathrm{C} \times \mathrm{CBA} / \mathrm{Ca}_{1} \mathrm{~F}_{1}$ hybrid male mice, mutations were scored at both the smaller BALB/c-derived allele ( $\sim 2.5 \mathrm{~kb})$ and the larger ( $\sim 3.3 \mathrm{~kb}$ ) CBA/Ca allele. Similar to previous studies of mutation detection at ESTR loci (Yauk et al., 2002; Glen et al., 2008; Abouzeid Ali et al., 2012; Glen \& Dubrova, 2012; Mughal et al., 2012; Voutounou et al., 2012), only novel DNA fragments demonstrating a shift of at least $1 \mathrm{~mm}$ comparative to the progenitor allele were scored as de novo ESTR mutants (Figure 13). Given that the Ms6-hm locus consists of a pentamer repeat sequence (GGGCA) (Kelly et al., 1989), a $1 \mathrm{~mm}$ shift in distance equates to either a gain (insertion) or loss (deletion) of three repeat units for the $\mathrm{CBA} / \mathrm{Ca}$ allele and two repeat units for the smaller BALB/C allele. Smaller lengthchanges however were not scored as they could not be consistently or reliably scored. All mutants pertaining to each tissue, exposure dose and time point, along with the age-matched sham-treated controls and X-ray irradiated males were reliably scored through two independent markers. Thereafter, the frequencies of ESTR mutations $(\mu)$ were calculated for each group (Equation 7), dividing the number of mutants, $m$ by the total number of amplifiable DNA molecules, $n$. The standard error of mutation frequency, se $\mu$ was estimated using Equation 7 : 


\section{Equation 7}

$$
\operatorname{se} \mu=\mu \sqrt{\left(\frac{\operatorname{sen}}{n}\right)^{2}+\frac{1}{m}}
$$

where sen is the standard error of the number of amplifiable DNA molecules. The Student's $t$ test was used to compare the frequency of ESTR mutation in exposed and sham treated mice.

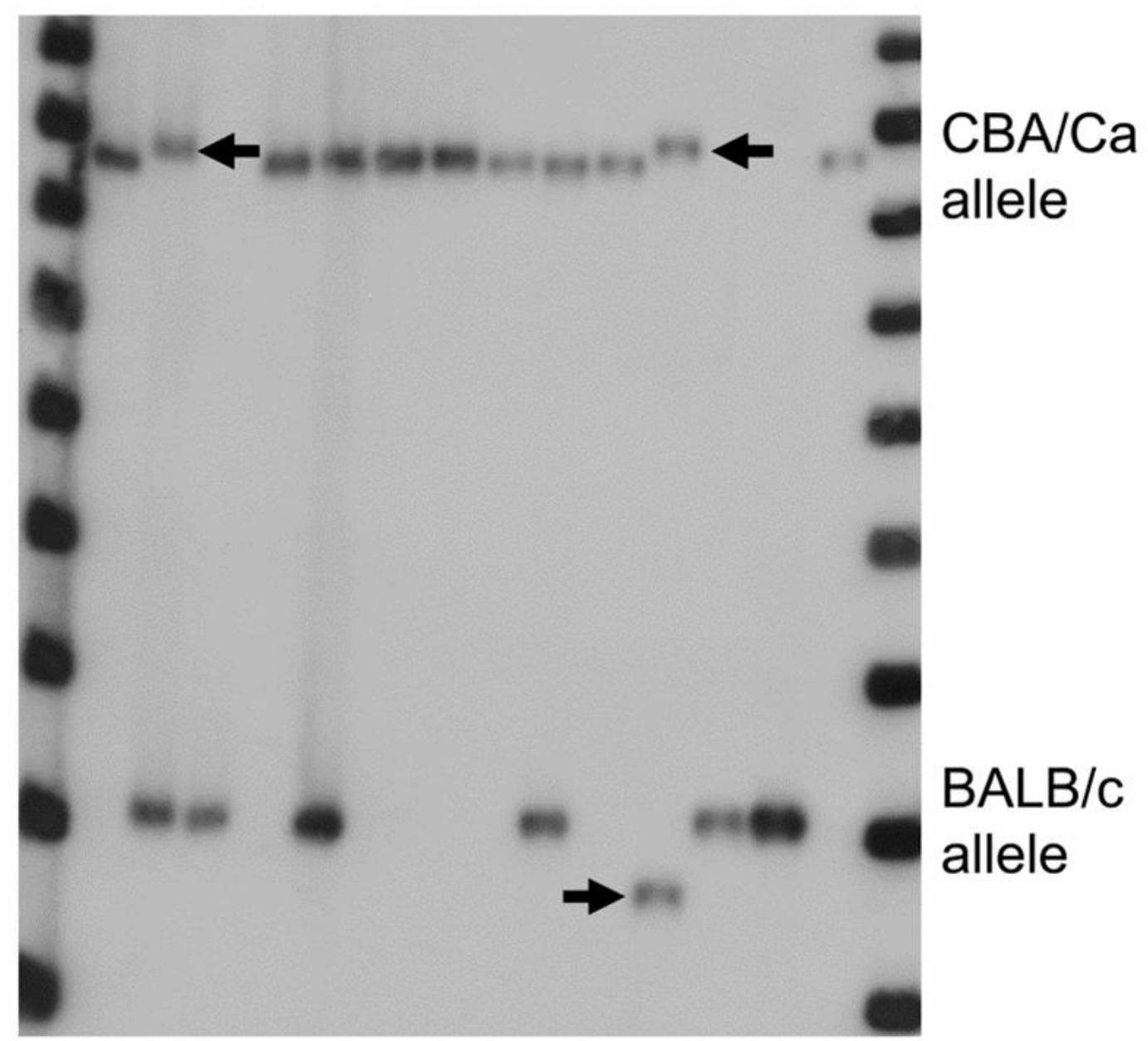

Figure 13. Mutation detection at the Ms6-hm ESTR locus. Southern blot analysis of the CBA/CA and BALB/C alleles at the Ms6-hm locus. Single molecule, positive reactions were identified and electrophoresed on a $40 \times 20 \mathrm{~cm}, 1 \%$ agarose gel. Bands assigned as mutants are indicated by arrows (CBA/Ca mutants, shifts of 1 \& 2 millimetres represent insertions of repeats; $B A L B / c$ mutant is indicative of a deletion). 200 bp DNA step ladder (Promega) is used as a side lane reference. 


\subsection{Mutation Mosaicism}

Due to the extremely high spontaneous mutation rate demonstrated by the Ms6-hm and $\mathrm{Hm}-2 \mathrm{ESTR}$ loci, the occurrence of both germline and somatic mutational mosaicism is especially prominent, with at least $3 \%$ of mice displaying an additional non-parental mutant allele in up to $60 \%$ of somatic cells (Yauk et al., 2002). Mosaics represent a cluster of identical mutations detected in multiple germline and somatic DNA samples which have arisen in the same cell during the first few cell divisions following fertilisation (Figure 14), (Kelly et al., 1989; Gibbs et al., 1993). Hence the presence of mosaics is not indicative of separate mutational events but rather the propagation of a single mutational event. In previous studies, typically those implementing a pedigree-based approach (Dubrova et al., 1998a; Barber et al., 2009), the presence of mosaics have presented issues during the scoring of mutations as the statistical power is too low to facilitate the detection of mutation rate heterogeneity between individuals (Barber et al., 2009).

Furthermore, it has been previously proposed that the presence of mosaics implies that robust estimates of mutation frequency cannot be derived from single animals. Instead, at least three mice must be analysed to test for inter-animal rate homogeneity and therefore lack of mosaicism (Yauk et al., 2002). Thus, to prevent mosaicism compromising the scoring of mutations in this study, blood and sperm samples of five $\mathrm{BALB} / \mathrm{c} \times \mathrm{CBA} / \mathrm{Ca} \mathrm{F}_{1}$ hybrid male mice were analysed for each ELF-field flux density (10 $\mu \mathrm{T}, 100 \mu \mathrm{T}$ or $300 \mu \mathrm{T}$ ) for 2 or 15 hours, in addition to those from the control groups (Table 14). While, as in previous studies (Dubrova et al., 1998a, 2000a; Voutounou et al., 2012), all cases of mosaicism within de novo mutations detected in multiple DNA samples, were collectively recorded and scored as a single independent mutation event. Moreover, the presence of a third non-parental allele within somatic samples was disregarded and not included in the mutation analysis. 


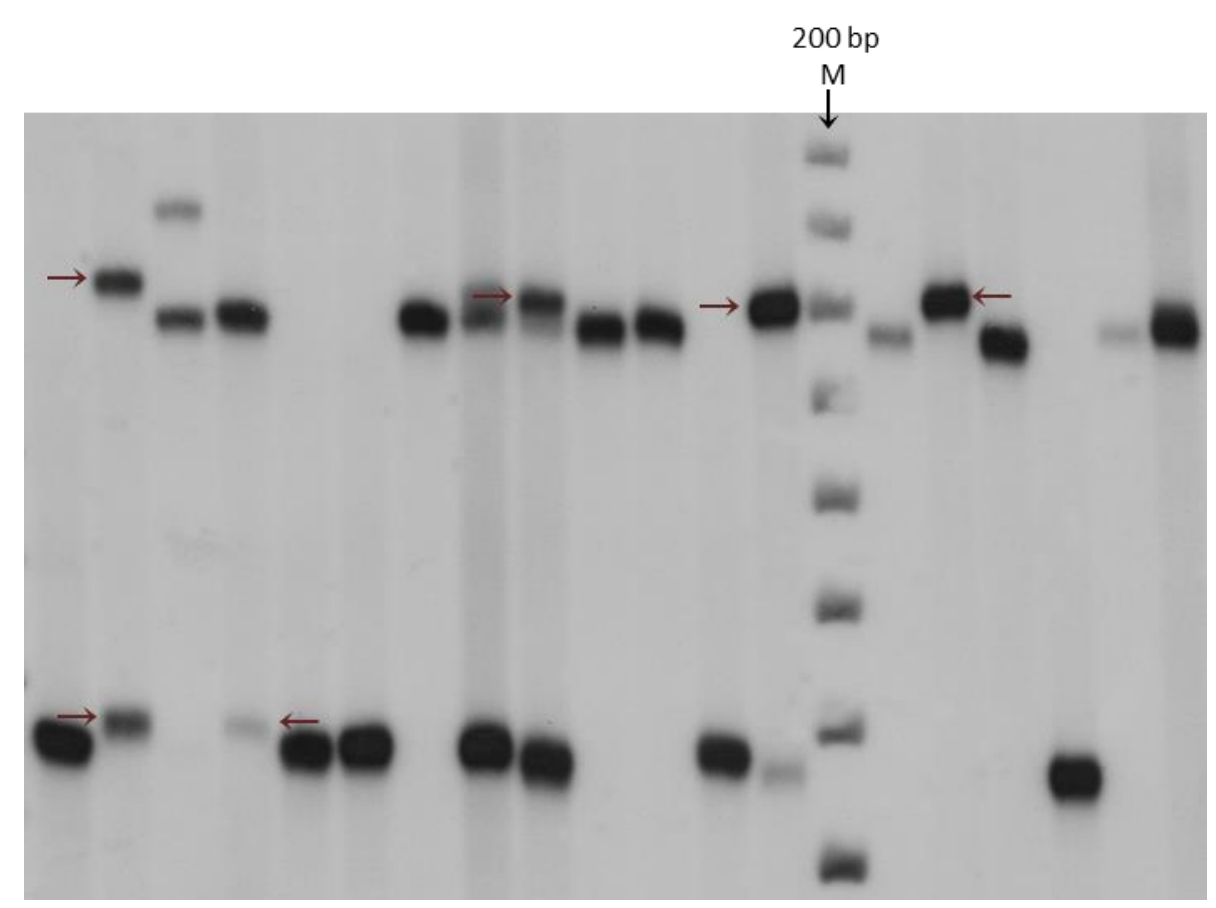

Figure 14. Mutational mosaicism at the Ms6-hm ESTR locus. Southern blot analysis of the CBA/CA and BALB/c alleles at the Ms6-hm locus. An autoradiograph representing 4 identical mosaic mutants at the CBA/CA allele and two mutants at the BALB/c (mutants indicated by arrows). 200 bp DNA step ladder (Promega) is used as a side lane reference.

\subsection{ESTR Mutation Frequencies in Sham-treated Males}

Initially, the ESTR mutation frequencies were established in the blood and sperm DNA samples of the three groups of age-matched sham-treated male mice. A summary of the mutational data for both blood and sperm is presented in Table 15. Additionally, the mean ESTR mutation frequencies calculated in blood and sperm are presented by Figure 15.

Table 15. ESTR mutation frequencies in sham-treated males

\begin{tabular}{|c|c|c|}
\hline Group & Number of mutations ${ }^{*}$ & Frequency \pm s.e. \\
\hline \multicolumn{3}{|c|}{ Sham-treated (Blood) } \\
\hline $2 \mathrm{~h}$ & $25(608 \pm 27)$ & $0.0411 \pm 0.0084$ \\
\hline $15 \mathrm{~h}$ & $28(738 \pm 30)$ & $0.0379 \pm 0.0073$ \\
\hline X-rays & $32(683 \pm 29)$ & $0.0468 \pm 0.0085$ \\
\hline All sham-treated & $85(2030 \pm 50)$ & $0.0419 \pm 0.0047$ \\
\hline \multicolumn{3}{|c|}{ Sham-treated (Sperm) } \\
\hline $2 \mathrm{~h}$ & $22(479 \pm 24)$ & $0.0460 \pm 0.0101$ \\
\hline $15 \mathrm{~h}$ & $36(731 \pm 30)$ & $0.0507 \pm 0.0054$ \\
\hline X-rays & $36(646 \pm 28)$ & $0.0557 \pm 0.0096$ \\
\hline All sham-treated & $94(1856 \pm 48)$ & $0.0507 \pm 0.0054$ \\
\hline
\end{tabular}

* Number of amplifiable molecules ( \pm standard error (s.e.)) is given in brackets. 


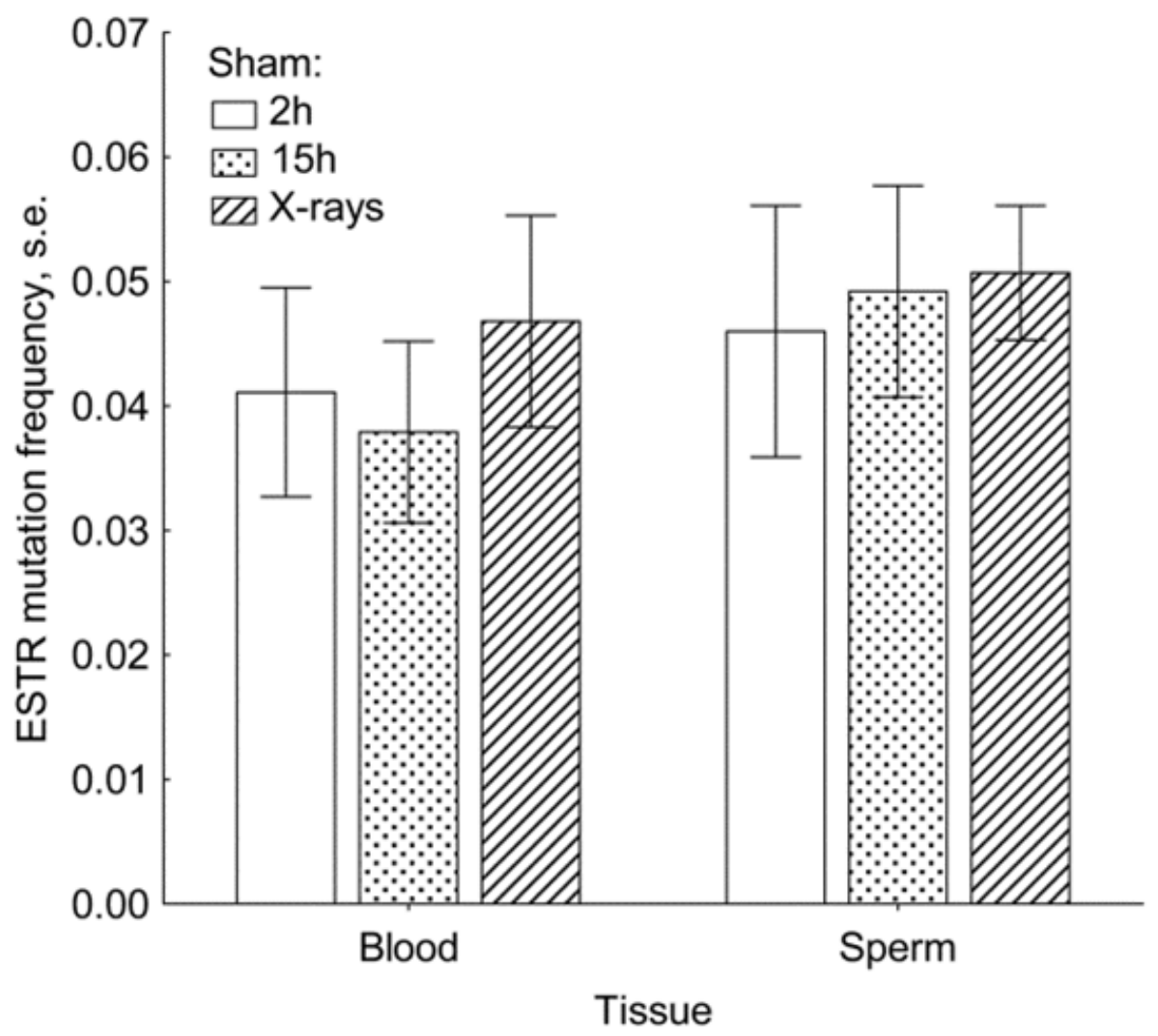

Figure 15. ESTR mutation frequencies at the Ms6-hm locus in sham-treated males. Mutations were scored in both tissues of each indicated sham-treated group. Standard error bars are shown.

A pairwise comparison performed between all the age-matched sham-treated control male mice in both tissues, determined that the frequency of ESTR mutation did not significantly differ between all sham-treated groups in either tissue (Table 16; blood, $0.43<P<0.77 ;$ sperm, $0.48<P 0.80$ ). Therefore, the age-matched sham-treated data were combined to form a single control group for each tissue. Such aggregation of all control data led to an increase in statistical power when comparing the mutation frequencies for all exposed males against those of the sham-treated males.

Table 16. Statistics for the difference between sham-treated groups

\begin{tabular}{|l|l|l|l|l|l|l|}
\hline & \multicolumn{5}{l}{ Sperm } & \multicolumn{2}{l|}{ Blood } \\
\hline Sham-treated & $\mathbf{2} \mathbf{h}$ & $\mathbf{1 5} \mathbf{h}$ & X-rays & $\mathbf{2} \mathbf{h}$ & $\mathbf{1 5} \mathbf{h}$ & X-rays \\
\hline $\mathbf{2} \mathbf{h}$ & - & $t=0.25$ & $t=0.70$ & - & $t=0.48$ & $t=0.29$ \\
\hline $\mathbf{1 5} \mathbf{h}$ & $P=0.8026$ & - & $t=0.51$ & $P=0.6313$ & - & $t=0.79$ \\
\hline X-rays & $P=0.4841$ & $P=0.6010$ & - & $P=0.7719$ & $P=0.4297$ & - \\
\hline
\end{tabular}

The Student's test $(t)$ and probability $(P)$ for the difference between the frequencies of ESTR mutation at the Ms6-hm locus in DNA samples extracted from blood and sperm of male mice that were sham-treated for either 2 hours, 15 hours or under the same conditions as the sample group exposed to $1 \mathrm{~Gy} \mathrm{X}$-rays. 


\subsection{ESTR Mutation Frequencies in Irradiated Males}

Five additional hybrid age-matched male mice were irradiated with an acute dose of 1 Gy X-rays in order to validate the SM-PCR technique (positive control). The frequency of ESTR mutation was significantly elevated in both the germline and somatic tissue of males exposed to $1 \mathrm{~Gy}$ of acute X-rays (Table 17). A highly significant 2.4-fold elevation in the frequency of Ms6-hm mutants was identified in sperm of irradiated males. Meanwhile, in blood, a less pronounced but still significant 1.8-fold increase was exhibited in these males following exposure.

Table 17. ESTR mutation frequencies for all age-matched controls

\begin{tabular}{|c|c|c|c|c|c|}
\hline Group & No mutations ${ }^{*}$ & Frequency \pm s.e. & $\begin{array}{l}\text { Ratio to } \\
\text { control }\end{array}$ & $t$ & Probability \\
\hline \multicolumn{6}{|c|}{ Sham-treated (Blood) } \\
\hline X-rays & $32(683 \pm 29)$ & $0.0468 \pm 0.0085$ & & & \\
\hline All sham-treated & $85(2030 \pm 50)$ & $0.0419 \pm 0.0047$ & & & \\
\hline X-rays, 1Gy & $54(706 \pm 30)$ & $0.0764 \pm 0.0109$ & $\begin{array}{l}1.63 \\
1.83\end{array}$ & $\begin{array}{l}2.14^{\dagger} \\
2.92^{\ddagger}\end{array}$ & $\begin{array}{l}0.2275 \\
0.0245\end{array}$ \\
\hline \multicolumn{6}{|c|}{ Sham-treated (Sperm) } \\
\hline X-rays & $36(646 \pm 28)$ & $0.0557 \pm 0.0096$ & & & \\
\hline All sham-treated & $94(1856 \pm 48)$ & $0.0507 \pm 0.0054$ & & & \\
\hline X-rays, 1Gy & $75(615 \pm 27)$ & $0.1220 \pm 0.0151$ & $\begin{array}{l}2.19 \\
2.41\end{array}$ & $\begin{array}{l}3.71^{\dagger} \\
4.46^{\ddagger}\end{array}$ & $\begin{array}{l}0.0014 \\
5.99 \times 10^{-5}\end{array}$ \\
\hline
\end{tabular}

The total number of unique mutations and the subsequent mutation frequencies in blood and sperm for the sham-treated male mice as well as those exposed to $1 \mathrm{~Gy}$ of acute X-rays. "Number of amplifiable molecules ( \pm s.e.) is given in brackets. ${ }^{\dagger}$ Student's test and Bonferroni corrected probability for difference from mutation frequency in the exposure-matched control group. ${ }^{\ddagger}$ Student's test and Bonferroni corrected probability for difference from mutation frequency in the total control group (all shamtreated).

\subsection{ESTR Mutation Frequencies in Males Exposed to $50 \mathrm{~Hz}$ Magnetic Fields}

\subsubsection{Blood}

Upon establishing the baseline mutation frequencies and aggregating the blood and sperm data of sham-treated controls, the frequency of ESTR mutation induction was next established in blood samples of MF-exposed male mice. Five BALB/c x CBA/Ca $F_{1}$ hybrid male mice were analysed for each magnetic field flux density $(10,100$ or 300 $\mu T$ ) at both time points of exposure (2 hours or 15 hours). The ESTR mutation data 
regarding the blood samples are summarised in Table 18, while Figure 16 presents the frequency of ESTR mutations in blood for all males exposed to ELF-MFs and the matched sham-treated males.

Table 18. Summary of ESTR mutation data in blood

\begin{tabular}{|l|l|l|l|l|l|}
\hline Group & No mutations & Frequency \pm s.e. & $\begin{array}{l}\text { Ratio to } \\
\text { control }\end{array}$ & $t$ & Probability \\
\hline Sham-treated & $25(608 \pm 27)$ & $0.0411 \pm 0.0084$ & & & \\
\hline $2 \mathrm{~h}$ & $28(738 \pm 30)$ & $0.0379 \pm 0.0073$ & & & \\
\hline $15 \mathrm{~h}$ & $85(2030 \pm 50)$ & $0.0419 \pm 0.0047$ & - & - & - \\
\hline $\begin{array}{l}\text { All sham- } \\
\text { treated }\end{array}$ & \multicolumn{5}{|l|}{} \\
\hline ELF-exposed & $24(623 \pm 27)$ & $0.0385 \pm 0.0080$ & 0.94 & $0.22^{\dagger}$ & 1 \\
\hline $10 \mu \mathrm{T}, 2 \mathrm{~h}$ & & & 0.92 & $0.36^{\ddagger}$ & 1 \\
\hline $10 \mu \mathrm{T}, 15 \mathrm{~h}$ & $26(575 \pm 26)$ & $0.0452 \pm 0.0091$ & 1.19 & $0.62^{\dagger}$ & 1 \\
& & & 1.08 & $0.33^{\ddagger}$ & 1 \\
\hline $100 \mu \mathrm{T}, 2 \mathrm{~h}$ & $26(527 \pm 25)$ & $0.0494 \pm 0.0100$ & 1.20 & $0.63^{\dagger}$ & 1 \\
& & & 1.18 & $0.68^{\ddagger}$ & 1 \\
\hline $100 \mu \mathrm{T}, 15 \mathrm{~h}$ & $30(597 \pm 27)$ & $0.0503 \pm 0.0095$ & 1.33 & $1.03^{\dagger}$ & 1 \\
& & & 1.20 & $0.80^{\ddagger}$ & 1 \\
\hline $300 \mu \mathrm{T}, 2 \mathrm{~h}$ & $23(624 \pm 27)$ & $0.0369 \pm 0.0079$ & 0.90 & $0.37^{\dagger}$ & 1 \\
& & & 0.88 & $0.55^{\ddagger}$ & 1 \\
\hline $300 \mu \mathrm{T}, 15 \mathrm{~h}$ & $27(709 \pm 30)$ & $0.0381 \pm 0.0075$ & 1.00 & $0.01^{\dagger}$ & 1 \\
& & & 0.91 & $0.43^{\ddagger}$ & 1 \\
\hline All ELF-MF, 2h & $73(1744 \pm 46)$ & $0.0412 \pm 0.0049$ & 0.98 & 0.11 & 1 \\
\hline All ELF-MF, 15h & $83(1881 \pm 48)$ & $0.0441 \pm 0.0050$ & 1.05 & 0.33 & 1 \\
\hline All ELF-MF & $156(3655 \pm 67)$ & $0.0427 \pm 0.0035$ & 1.02 & 0.14 & 0.8887 \\
\hline & & & & & \\
\hline
\end{tabular}

* Number of amplifiable molecules ( \pm s.e.) is given in brackets. ${ }^{+}$Student's test and Bonferroni corrected probability for difference from mutation frequency in the exposure-matched control group. ${ }^{\ddagger}$ Student's test and Bonferroni corrected probability for difference from mutation frequency in the total control group (all sham-treated).

Taken together, these data demonstrate that the frequency of ESTR mutation in the somatic tissue of all males exposed to magnetic fields did not significantly differ from that of their equivalent sham-treated control groups. Furthermore, a comparison of ESTR mutation frequencies of all exposed males against the aggregated sham-treated group are in accordance with those of their age-matched equivalent, and again fail to reveal any significant differences. 


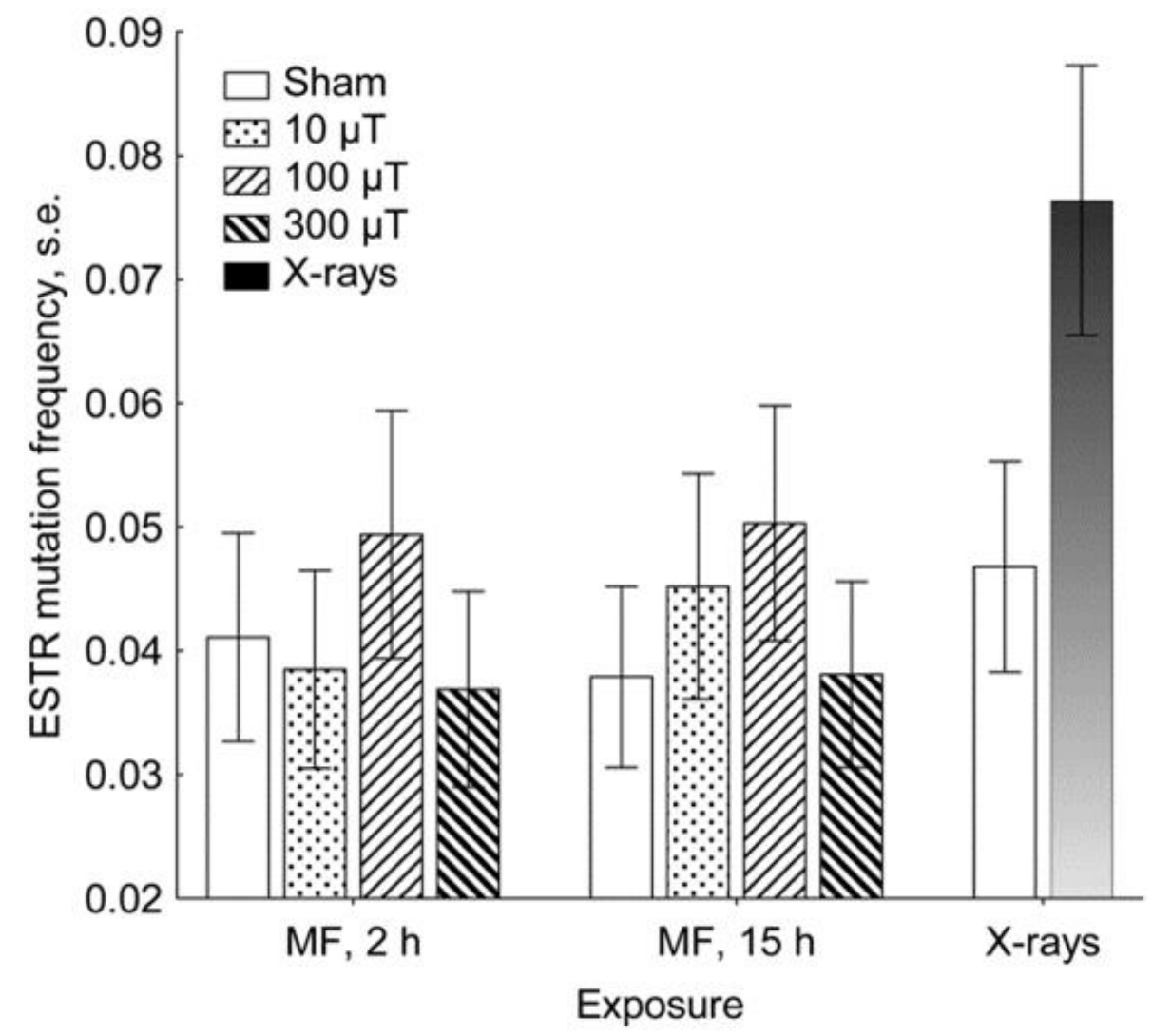

Figure 16. ESTR mutation frequencies in blood of exposed and matched shamtreated males. SM-PCR and Southern blot analysis were performed on DNA extracted from the blood of exposed CBA/CA x BALB/c male mice. Mutations were scored for each of the indicated exposure doses/time periods. Standard error bars are shown.

Thereafter, the effects of both 2 hours and 15 hours exposure to $50 \mathrm{~Hz}$ magnetic fields of $10 \mu \mathrm{T}, 100 \mu \mathrm{T}$ or $300 \mu \mathrm{T}$ in strength were established in the germline of BALB/c $\mathrm{x}$ $\mathrm{CBA} / \mathrm{Ca} \mathrm{F}_{1}$ hybrid male mice.

\subsubsection{Sperm}

Again mutations were scored in five hybrid male mice per group. The ESTR mutation data for sperm are summarised in Table 19.

While, the frequency of ESTR mutation in sperm of all exposed males exceeds that illustrated in blood, no significant differences were revealed between ESTR mutation frequencies of the exposed males and their equivalent sham-treated counterparts (Figure 17; $0.52<P \leq 1$ for all pair wise comparisons). These findings were again corroborated through a comparison of mutation frequencies against the aggregated sham-treated control data. Again, the frequency of ESTR mutation in the germline 
tissue of all males exposed to magnetic fields did not significantly differ from that in the total control group $(0.45<P \leq 1$ for all pair wise comparisons).

Table 19. Summary of ESTR mutation data in sperm

\begin{tabular}{|c|c|c|c|c|c|}
\hline Group & No mutations ${ }^{*}$ & Frequency \pm s.e. & $\begin{array}{l}\text { Ratio to } \\
\text { control }\end{array}$ & $t$ & Probability \\
\hline \multicolumn{6}{|l|}{ Sham-treated } \\
\hline $2 \mathrm{~h}$ & $22(479 \pm 24)$ & $0.0460 \pm 0.0101$ & & & \\
\hline $15 \mathrm{~h}$ & $36(731 \pm 30)$ & $0.0507 \pm 0.0054$ & & & \\
\hline All sham-treated & $94(1856 \pm 48)$ & $0.0507 \pm 0.0054$ & - & - & - \\
\hline \multicolumn{6}{|l|}{ ELF-exposed } \\
\hline $10 \mu \mathrm{T}, 2 \mathrm{~h}$ & $45(639 \pm 29)$ & $0.0704 \pm 0.0110$ & $\begin{array}{l}1.53 \\
1.39\end{array}$ & $\begin{array}{l}1.64^{\dagger} \\
1.62^{\ddagger}\end{array}$ & $\begin{array}{l}0.7091 \\
0.7378\end{array}$ \\
\hline $10 \mu \mathrm{T}, 15 \mathrm{~h}$ & $38(621 \pm 27)$ & $0.0612 \pm 0.0103$ & $\begin{array}{l}1.24 \\
1.21\end{array}$ & $\begin{array}{l}0.90^{\dagger} \\
0.91^{\ddagger}\end{array}$ & $\begin{array}{l}1 \\
1\end{array}$ \\
\hline $100 \mu \mathrm{T}, 2 \mathrm{~h}$ & $49(852 \pm 33)$ & $0.0575 \pm 0.0085$ & $\begin{array}{l}1.25 \\
1.13\end{array}$ & $\begin{array}{l}0.87^{+} \\
0.68^{\ddagger}\end{array}$ & $\begin{array}{l}1 \\
1\end{array}$ \\
\hline $100 \mu \mathrm{T}, 15 \mathrm{~h}$ & $59(829 \pm 32)$ & $0.0712 \pm 0.0097$ & $\begin{array}{l}1.45 \\
1.40\end{array}$ & $\begin{array}{l}1.71^{\dagger} \\
1.85^{\ddagger}\end{array}$ & $\begin{array}{l}0.6125 \\
0.4508\end{array}$ \\
\hline $300 \mu \mathrm{T}, 2 \mathrm{~h}$ & $59(833 \pm 33)$ & $0.0708 \pm 0.0096$ & $\begin{array}{l}1.54 \\
1.40\end{array}$ & $\begin{array}{l}1.78^{\dagger} \\
1.83^{\ddagger}\end{array}$ & $\begin{array}{l}0.5271 \\
0.4718\end{array}$ \\
\hline $300 \mu \mathrm{T}, 15 \mathrm{~h}$ & $52(855 \pm 33)$ & $0.0609 \pm 0.0088$ & $\begin{array}{l}1.24 \\
1.20\end{array}$ & $\begin{array}{l}0.95^{\dagger} \\
0.99^{\ddagger}\end{array}$ & $\begin{array}{l}1 \\
1\end{array}$ \\
\hline All ELF-MF, $2 \mathrm{~h}$ & $153(2324 \pm 55)$ & $0.0658 \pm 0.0055$ & 1.30 & 1.96 & 0.1002 \\
\hline All ELF-MF, $15 \mathrm{~h}$ & $146(2280 \pm 54)$ & $0.0640 \pm 0.0055$ & 1.26 & 1.74 & 0.1638 \\
\hline All ELF-MF & $299(4604 \pm 76)$ & $0.0649 \pm 0.0039$ & 1.28 & 2.15 & 0.0316 \\
\hline
\end{tabular}

* Number of amplifiable molecules ( \pm s.e.) is given in brackets. ${ }^{\dagger}$ Student's test and Bonferroni corrected probability for difference from mutation frequency in the exposure-matched control group. ${ }^{\ddagger}$ Student's test and Bonferroni corrected probability for difference from mutation frequency in the total control group (all sham-treated).

Overall, the frequency of ESTR mutation in the germline and blood of all exposed males did not significantly differ from that in their equivalent sham-treated groups (Figures 16, 17; $0.52<P \leq 1$ for all pair wise comparisons). Nor was there any significant difference when the frequency of ESTR mutation in the blood and sperm of all exposed males were compared to that of an aggregated total control group. 


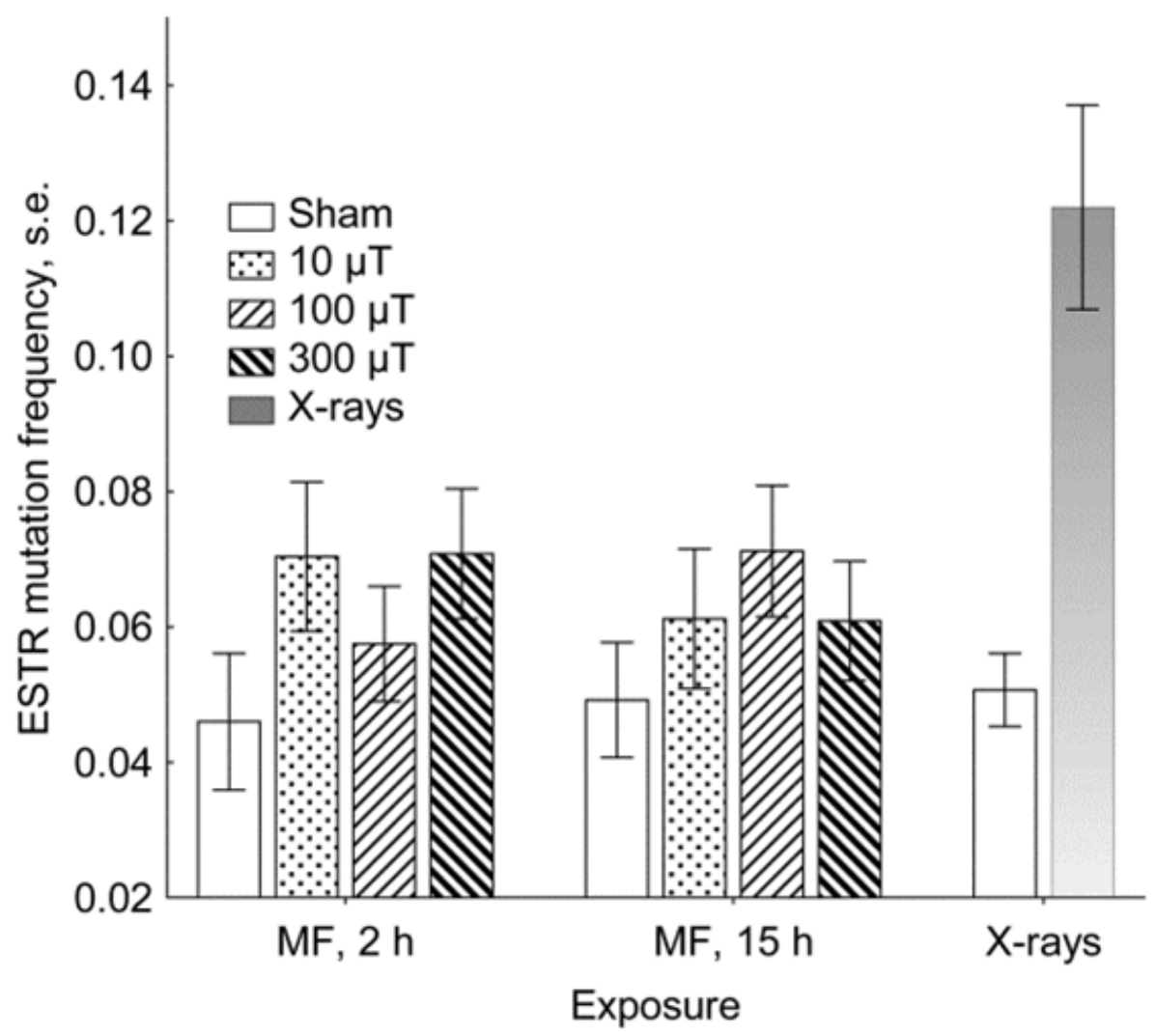

Figure 17. ESTR mutation frequencies in sperm of exposed and matched shamtreated males. SM-PCR and Southern blot analysis were performed on DNA extracted from the sperm of exposed CBA/CA x BALB/c male mice. Mutations were scored for each of the indicated exposure doses/time periods. Standard error bars are shown.

\subsubsection{Combined ELF-MF effect}

To increase the statistical power of the present findings, all the ESTR mutation data for all exposed mice were pooled to include all exposure conditions (field flux density and exposure length), for each individual tissue (Tables 18, 19). Additionally, in the present study, the ESTR mutation frequency of the pooled data was analysed according to the length of exposure for each tissue to determine any cumulative effects of exposure for either 2- or 15 hours.

Upon aggregation of all blood data, the ESTR mutation frequency did not significantly differ and was found to be only marginally elevated when compared to that in shamtreated animals $(P=0.8887)$. While analysis of pooled data for each individual exposure length (2- or 15 hours) also presented similar findings, analysis of the aggregated sperm data illustrated a marginally significant elevation in the total frequency of ESTR mutation $(P=0.0316)$. Meanwhile, separate analysis of each individual exposure length revealed virtually no discernible difference in total mutation 
frequencies between males exposed for 15 hours and those irradiated for a duration of 2 hours (Figure $18 ; 2$ hours, $P=0.1002 ; 15$ hours, $P=0.1638$ ).

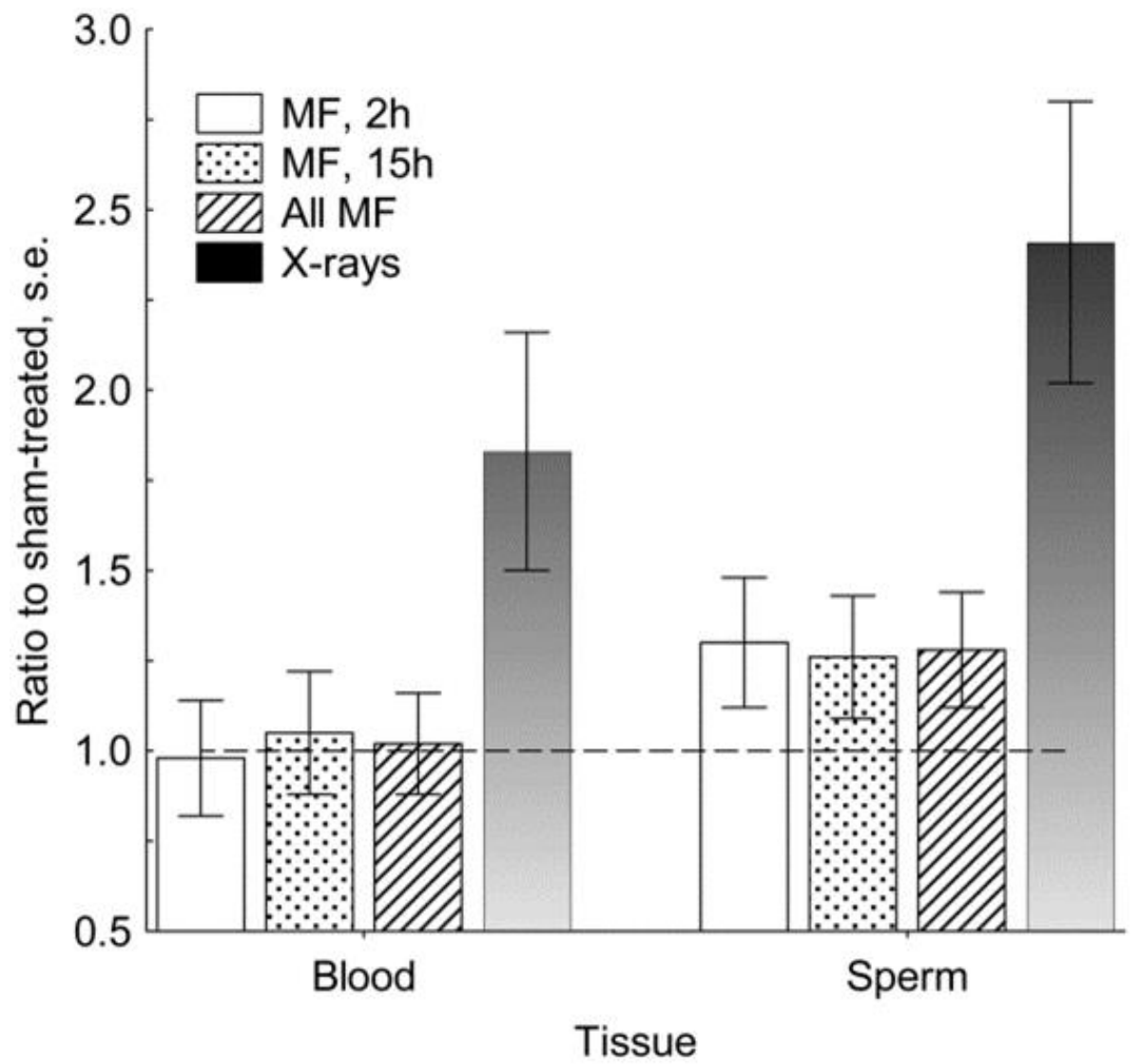

Figure 18. ESTR mutation frequencies in sham-treated and exposed males. $A$ comparison of the average fold induction of ESTR mutation frequencies for both blood and sperm of exposed mice relative to the control. Standard errors are shown.

\subsection{Mutation Spectrum}

Following ESTR mutation frequency analysis, the spectra of length-changes were next examined in each of the identified induced and spontaneous Ms6-hm mutants. According to previous studies, Ms6-hm mutants exhibit similar mutational spectra irrespective of whether mutations arose spontaneously or were induced by ionising radiation (Yauk et al., 2002) or chemical mutagens (Glen et al., 2008). Similarly, no significant differences in the size distribution of mutants were found to arise between Ms6-hm mutations in the germline or somatic tissues taken from 8-12 week old male mice (Yauk et al., 2002; Barber et al., 2006, 2009; Hatch et al., 2007). These data therefore suggest that mutations arising in germline or somatic tissues, either spontaneously or induced, do so through a similar mutation process previously theorised to be through replication slippage. 
Therefore, to further substantiate the aforementioned working hypothesis, the spectra for all 757 de novo ESTR mutant molecules recovered in blood and sperm were analysed. As in previous studies (Yauk et al., 2002; Glen et al., 2008; Abouzeid Ali et al., 2012; Voutounou et al., 2012), analysis involved the categorisation of Ms6-hm mutants as either insertions or deletions, as well as the determination of the number of repeat units gained or lost at each locus. As with mutational analysis, those mutants exhibiting mosaicism were treated as a single mutational event and subsequently analysed as one.

All Ms6-hm mutants identified in blood samples exhibiting repeat unit insertion following ELF-MF exposure were aggregated, irrespective of exposure parameters, as were those presenting repeat unit deletions. These measures were undertaken to increase the statistical power in the analysis of the distribution of repeat units gained or lost by the identified Ms6-hm mutants following ELF-MF exposure.

\subsubsection{Size Spectra of Ms6-hm Mutations in Blood}

The mutation distributions were initially analysed for blood and a summary of the mutation spectrums for the 291 de novo mutations detected is presented in Table 20.

Table 20. ESTR mutation spectra of control and treated males in blood

\begin{tabular}{|l|l|l|l|}
\hline Group & Gains (\%) & Losses (\%) & Total \\
\hline Control & $62(72.94 \%)$ & $23(27.06 \%)$ & 85 \\
MF & $98(64.47 \%)$ & $54(35.53 \%)$ & 152 \\
X-rays & $40(74.07 \%)$ & $14(25.93 \%)$ & 54 \\
Total & $200(68.73 \%)$ & $91(31.27 \%)$ & 291 \\
\hline Chi-square all & $\chi^{2} \mathrm{df}=2,=2.70$ & $P=0.2592$ & \\
\hline Chi-square MF & $\chi^{2} \mathrm{df}=1,=1.78$ & $P=0.1819$ & \\
\hline Chi-square X-rays & $\chi^{2} \mathrm{df}=1,=0.02$ & $P=0.8829$ & \\
\hline
\end{tabular}

The incidence of unique mutations involving gain and loss of repeat units at the Ms6$\mathrm{hm}$ locus detected in DNA samples extracted from blood of control and exposed male mice. The chi-square test and corresponding $P$ values for differences between groups are also shown.

Individual analysis into the distribution of mutant alleles (gain vs loss of repeat units) for either ELF-MF or X-ray samples revealed no significant differences to those exhibited by the sham-treated controls (Table 20; ELF-MF, $P=0.1819 ; \mathrm{X}$-rays, $P=$ 0.8829). Likewise, although demonstrating a slight visible preference towards the gain of repeat units, there was no significant difference in the combined distributions of 
mutations involving gain or loss of repeat units between the irradiated (MF \& X-ray) and control groups $(P=0.2592)$ in blood samples.

Upon characterising the distribution of the identified Ms6-hm mutants, the spectra of length-changes involved in both mutant additions and deletions were next considered (Figure 19). The same process was followed as in Yauk et al. (2002), in which mutations involving insertions or deletions of similar lengths were grouped \pm 2-5 repeats, \pm 6-9 repeats, $\pm 10-13, \pm 14-29$ and $\pm 30+$ repeats). The spectra of size changes in Ms6-hm mutants were compared between blood samples taken from unexposed sham-treated, ELF-MF exposed and X-ray irradiated male mice. The combined distributions of lengthchanges at ESTR loci did not differ significantly $(P=0.0819)$, indicating that there is no divergence between the insertion and deletion of repeat units. 


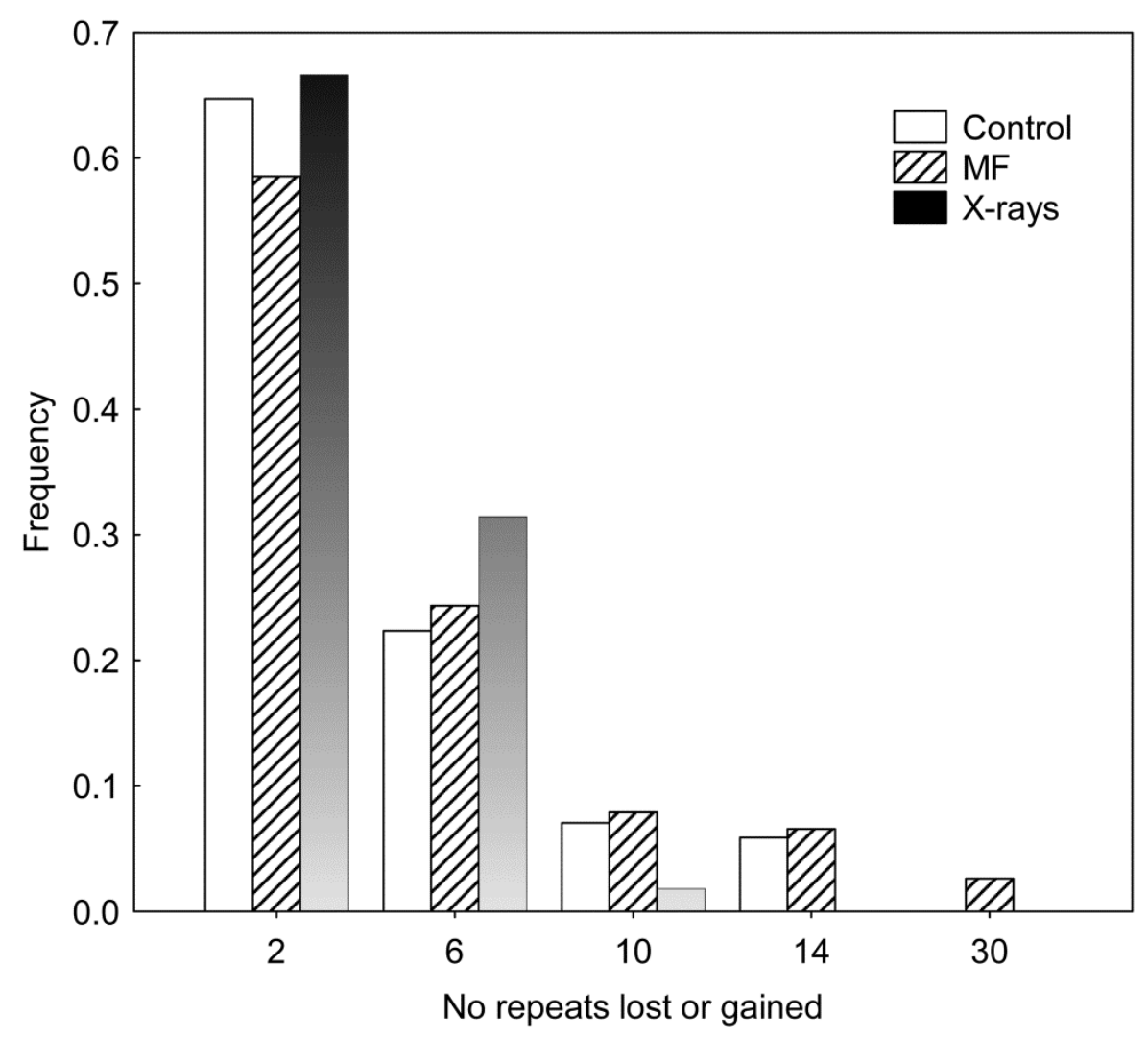

Figure 19. Spectra of somatic ESTR mutations in sham-treated controls and exposed male mice. The progenitor allele was assumed to be the parental allele closest in size to the mutant allele. Kruskal-Wallis test, $P=0.0819$.

\subsubsection{Size Spectra of Ms6-hm Mutations in Sperm}

The distribution and spectra of length-changes for all $466 \mathrm{Ms} 6-\mathrm{hm}$ germline mutations were also defined. A summary of the frequency distribution for gains and losses of Ms6-hm repeat units is presented in Table 21.

Comparable to blood samples, there were no significant differences in the combined distributions of mutations involving gain or loss of repeat units in sperm between the irradiated (ELF-MF \& X-ray) and control groups $(P=0.5541)$. Furthermore, the spectra of length-changes in the sperm Ms6-hm mutants did not significantly differ between all groups either (Figure 20; $P=0.8438$ ). 
Table 21. ESTR mutation spectra of control and treated males in sperm

\begin{tabular}{|l|l|l|l|}
\hline Group & Gains (\%) & Losses (\%) & Total \\
\hline Control & $52(55.32 \%)$ & $42(44.68 \%)$ & 94 \\
MF & $183(61.62 \%)$ & $114(38.38 \%)$ & 297 \\
X-rays & $45(60.00 \%)$ & $30(40.00 \%)$ & 75 \\
Total & $280(60.09 \%)$ & $186(39.91 \%)$ & 466 \\
\hline Chi-square all & $\chi^{2} \mathrm{df}=2,=1.18$ & $P=0.5541$ & \\
\hline Chi-square MF & $\chi^{2} \mathrm{df}=1,=1.18$ & $P=0.2772$ & \\
\hline Chi-square X-rays & $\chi^{2} \mathrm{df}=1,=0.37$ & $P=0.5409$ & \\
\hline
\end{tabular}

The incidence of unique mutations involving gain and loss of repeat units at the Ms6$\mathrm{hm}$ locus detected in DNA samples extracted from sperm of control and exposed male mice. The chi-square test and corresponding $P$ values for differences between groups are also shown.

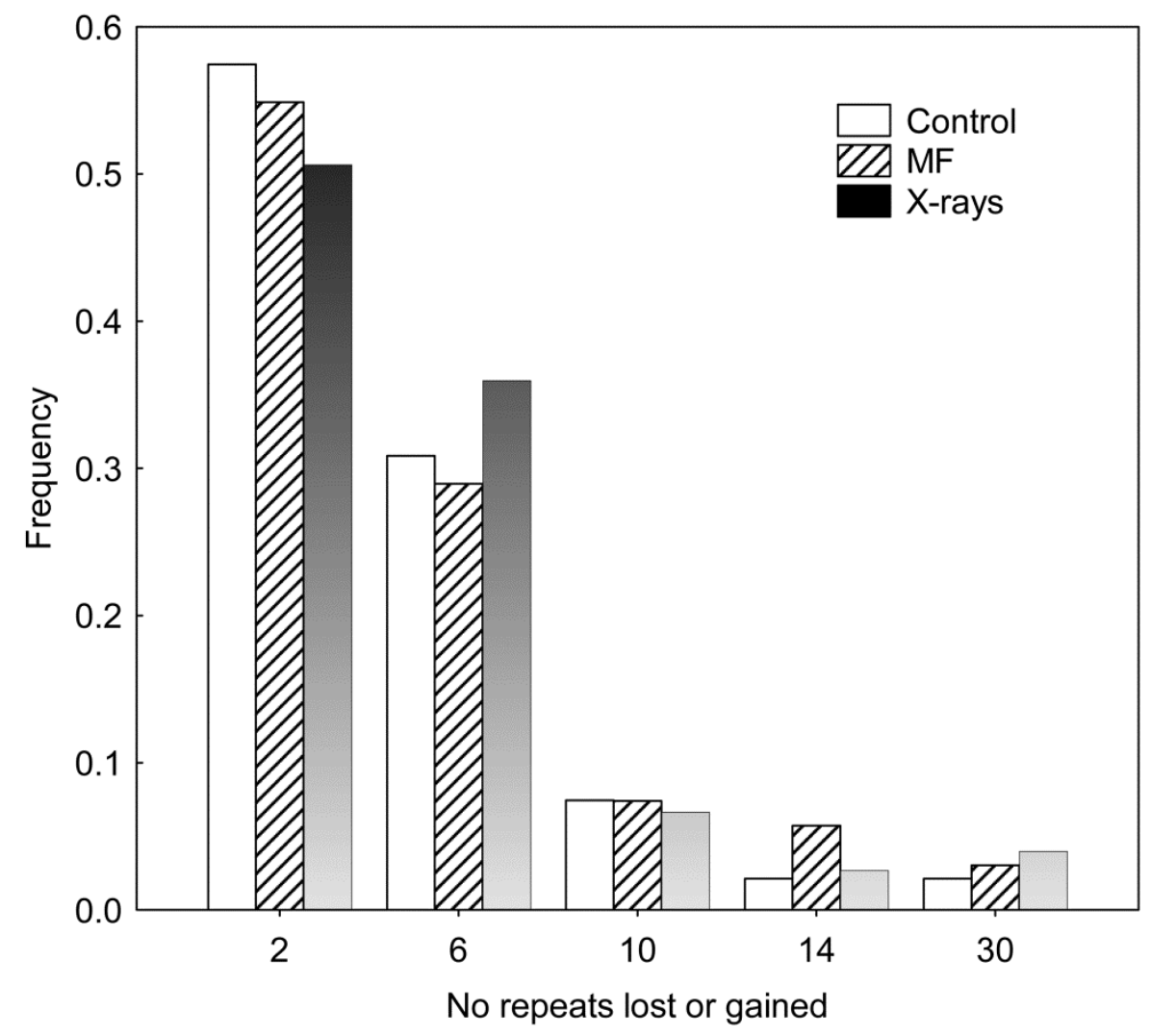

Figure 20. Spectra of germline ESTR mutations in sham-treated controls and exposed male mice. The progenitor allele was assumed to be the parental allele closest in size to the mutant allele. Kruskal-Wallis test, $P=0.8438$.

Overall, the data presented in the current study therefore indicate that neither the frequency distribution nor the spectra of length-changes in Ms6-hm mutants significantly alter from those found in age-matched sham-treated controls, in either the germline or somatic tissues of hybrid male mice exposed to a $50 \mathrm{~Hz}$ magnetic field at intensities of 10,100 and $300 \mu$ T for 2 - and 15 hours. Likewise, acute irradiation to 1 
Gy X-rays does not significantly change frequency distribution, or the spectra of lengthchanges in Ms6-hm mutants from those found in either germline or somatic tissues of age-matched sham-treated controls. The data from this present study are therefore in accordance with those previously presented (Yauk et al., 2002; Dubrova, 2005), and provide further evidence as to the presence of a similar mutational process within both the germline and soma in irradiated male mice (1 Gy acute X-rays).

\subsection{Microarray Pilot Study}

\subsubsection{Experimental Design}

While the present analysis of ESTR mutation frequencies failed to detect any significant changes between ELF-MF exposed and sham-treated males, ELF-MF represents a weak environmental stimulus incapable of ionising molecules, therefore any potential impact of ELF-MF on human health is likely complex. Thus the use of microarray technology to analyse gene expression profiles, following ELF-MF exposure, may not only identify prospective gene candidates but also provides an additionally informative endpoint in the series of ELF-MF studies. Considering this, this present pilot study was designed and initiated in an attempt to validate the mutation induction data. Gene expression profiles pertaining to ELF-MF and age-matched sham-treated BALB/C $\mathrm{x}$ $\mathrm{CBA} / \mathrm{Ca} \mathrm{F}_{1}$ hybrid male mice were compared in parallel with those from X-ray irradiated males using microarray expression data.

Five $\mathrm{BALB} / \mathrm{C} \times \mathrm{CBA} / \mathrm{Ca} \mathrm{F}_{1}$ hybrid male mice were exposed for 15 hours to a magnetic flux density of $300 \mu \mathrm{T}$ or whole-body acute irradiation of $1 \mathrm{~Gy}$ X-rays and their kidney samples taken 12 weeks post-exposure. Kidney samples were also taken from an additional five sham-treated BALB/C x CBA/Ca $F_{1}$ hybrid male mice 12 weeks post mock-exposure and used as control samples for data analysis. Indeed, the kidney represents a transcriptionally rich tissue in which to analyse the effects of ELF-MF on gene expression as it is a highly specialised organ with a great diversity of functions and cell types essential in numerous hormonal and homeostatic regulatory functions. Moreover, high quality RNA extraction and gene pattern analyses have previously been illustrated in non-exposed offspring of irradiated CBA/Ca and BALB/c male mice (Gomes et al., 2015) as well as in CBA/Ca and BALB/c mice subjected to a methyl-donor deficient diet (Glen et al., 2015). RNAs were therefore extracted from both kidneys in 
each mouse per exposure group and quality analysis performed (Chapter 2.13.1, Figure 10). Additionally, each individual RNA sample from the five male mice was subjected to three independent biological replicates while, to minimize inter-experiment variation, microarrays were performed in duplicate for each experimental group.

This preliminary study implemented a one-colour microarray-based gene expression analysis, whereby cyanine 3-labelled target sequences were used to measure gene expression profiles of MF-exposed and X-ray irradiated BALB/C $\times$ CBA/Ca $F_{1}$ hybrid males, with the highly sensitive Agilent SurePrint G3 Mouse GE 8x60K microarrays (Agilent Technologies, Santa Clara, CA, USA). Each Agilent SurePrint G3 Mouse GE $8 \times 60 \mathrm{~K}$ microarray slide was printed with eight identical arrays containing 39,430 Entrez Gene RNAs and 16,251 lincRNAs (long intergenic non-coding RNAs). Gene expression profiles were analysed using a one-colour microarray analysis which utilises an intensity-based platform, through which the intensities of each individual probe were generated in each array experiment. The use of a one-colour microarray analysis offered several advantages in that it allowed for inter-array comparisons of the measured gene expression, while the use of only cyanine 3 dye was more robust compared to the more degradation-prone cyanine 5 dye.

\subsubsection{Gene Expression Analysis}

\subsubsection{ELF-MF Gene Expression Analysis}

The pattern of gene expression was initially established in the ELF-MF exposed males (300 $\mu \mathrm{T}, 15$ hours). Microarray analysis was visualised primarily through volcano plots following the employment of the Student's $t$ test with false discovery rate (FDR) parameters of $P<0.05$ and identifying only those transcripts expressing a fold change of $>2$. The primary data did not reveal any differentially expressed loci following exposure to $300 \mu \mathrm{T}$ for 15 hours compared with the sham-treated controls (Figure 21). 


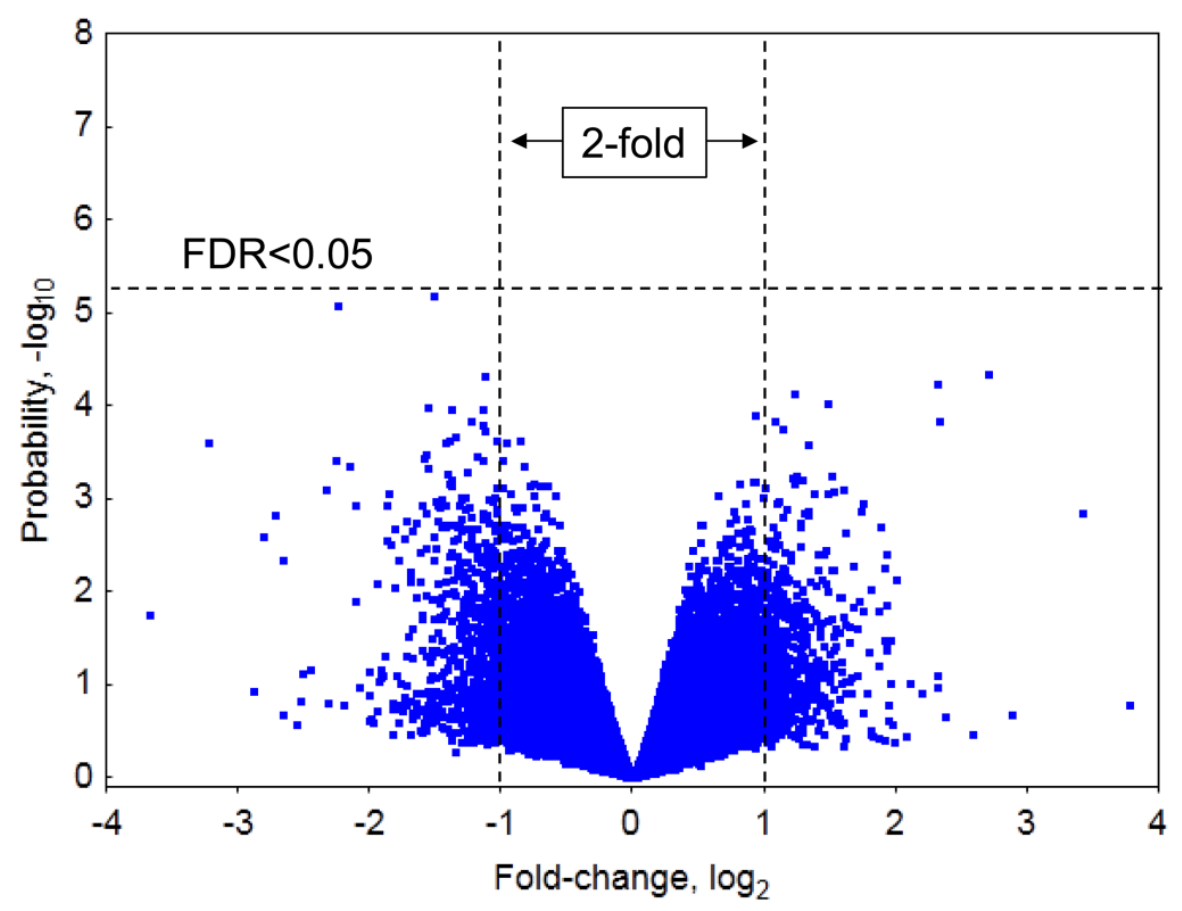

Figure 21. ELF-MF gene expression analysis. Volcano plot analysis exhibiting the differential pattern of gene expression in $\mathrm{BALB} / \mathrm{C} \times \mathrm{CBA} / \mathrm{Ca} \mathrm{F}_{1}$ hybrid male mice following 15 hour exposure to $300 \mu \mathrm{T}$ compared to their age-matched sham-treated controls. The $\log _{2}$ fold-change values are plotted on the $x$-axis of the volcano plots and are compared to the negative $\log _{10}$ raw $P$ values on the $y$-axis.

\subsubsection{Ionising Radiation Gene Expression Analysis}

Using the same two-fold cut-off criteria, the pattern of expression of protein-coding genes (mRNA) and long intergenic non-coding RNAs (lincRNAs) were analysed 12weeks post whole-body acute $1 \mathrm{~Gy}$ X-ray exposure (positive control). In contrast to ELF-MF gene expression profile analysis, RNA samples extracted from irradiated kidney cells revealed five significantly up-regulated loci $(P<0.05)$ when compared with shamtreated controls (shown in red, Figure 22). The five significantly up-regulated loci identified within the positive control experiment of this pilot study relate to the two genes; $/ 112 r b 2$ and $L t b 4 r 2$, in addition to three lincRNAs and are summarised in Table 22. 


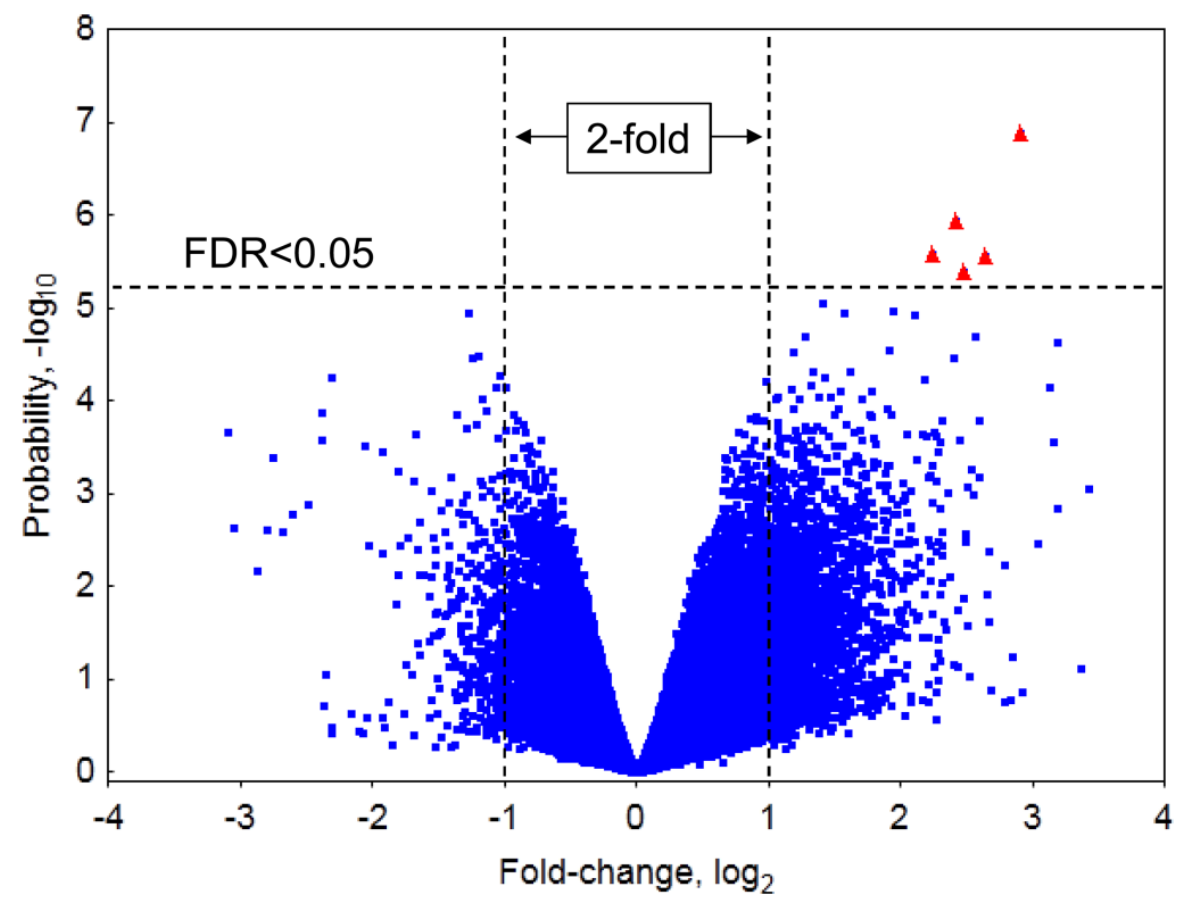

Figure 22. lonising radiation gene expression analysis. Volcano plot analysis exhibiting the differential pattern of gene expression in $\mathrm{BALB} / \mathrm{C} \times \mathrm{CBA} / \mathrm{Ca} \mathrm{F}_{1}$ hybrid male mice following whole-body acute $1 \mathrm{~Gy} X$-ray exposure compared to their age-matched sham-treated controls. The $\log _{2}$ fold-change values are plotted on the $x$-axis of the volcano plots and are compared to the negative $\log _{10}$ raw $P$ values on the $y$-axis. Genes with an absolute differential expression fold-change $>2$, alongside a raw $P$ value $<0.05$ are shown in red. 
Table 22. Summary of up-regulated genes

\begin{tabular}{|l|l|l|l|l|l|l|}
\hline Probe Name & Gene Symbol & Description & Fold Change & Log Fold Change & Regulation & $P($ Corr) \\
\hline A_30_P01018642 & & $\begin{array}{l}\text { lincRNA:chr17:14320464-14320975 } \\
\text { forward strand }\end{array}$ & 4.73 & Up & 0.0384 \\
\hline A_55_P1970489 & II12rb2 & $\begin{array}{l}\text { Mus musculus interleukin 12 receptor, } \\
\text { beta 2 (II12rb2), mRNA [NM_008354] }\end{array}$ & 6.25 & 2.64 & Up \\
\hline A_51_P282211 & Ltb4r2 & $\begin{array}{l}\text { Mus musculus leukotriene B4 receptor 2 } \\
\text { (Ltb4r2), mRNA [NM_020490] }\end{array}$ & 7.51 & 2.91 & Up & 0.0072 \\
\hline A_30_P01025210 & & $\begin{array}{l}\text { lincRNA:chr8:44232164-44267061 reverse } \\
\text { strand }\end{array}$ & 5.55 & 2.47 & Up & 0.0454 \\
\hline A_30_P01030493 & & $\begin{array}{l}\text { lincRNA:chr10:39659425-39674200 } \\
\text { forward strand }\end{array}$ & 5.33 & 2.42 & Up & 0.0323 \\
\hline
\end{tabular}

The details of expression changes exhibited by the five up-regulated probes following whole body acute exposure to $1 \mathrm{~Gy} X$-rays.

* Probability values were corrected for multiple testing using the Benjamini-Hochberg False Discovery Rate method. 
Next, the normalised Cy-3 intensity values were compared for each of the five upregulated probes between each X-ray irradiated and sham-treated male mice. These data confirmed an absence of outliers, illustrating that each probe was persistently upregulated in each of the five X-ray irradiated mice, and in contrast to the corresponding expression in the sham-treated males (Figure 23).

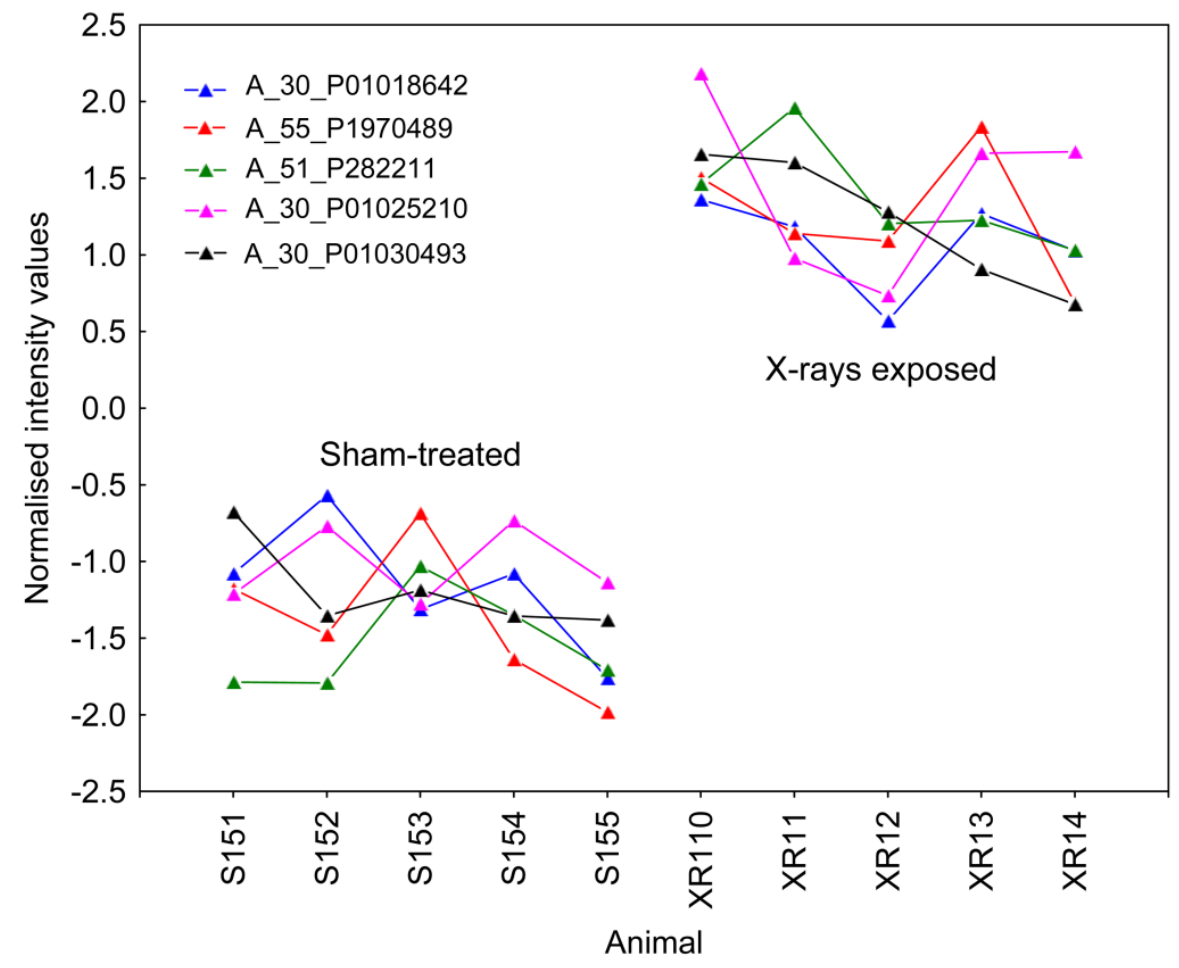

Figure 23. Comparison of differentially expressed probes. Line plot analysis illustrating the normalised signal intensities ( $\log _{2}$ values) of each up-regulated probe in each individual sham-treated and X-ray irradiated male mouse.

Finally, a probability plot was employed as an alternative exposure metric to confirm the prior analyses identifying the absence of statistically significant exposure effect in the ELF-MF exposed males, in addition to the significant upregulation of five transcripts in males irradiated with $1 \mathrm{~Gy}$ acute X-rays (Figure 24). Probability plots represent a useful method in preliminary microarray data analyses as it allows the correction for the large number of comparisons, thereby offering an informative and functionally useful interpretation for the thousands of genes being studied (Wartenberg et al., 1994). Normally implemented to evaluate how well a data set matches a specific distribution, in this current study the probability plot has been utilised to represent a visual aid in the confirmation of genes with differential expression levels. Owing to such a large number of comparisons, genes whose 
expression levels vary between the control and treatment groups can be identified as the points that deviate significantly from the otherwise linear relationship. For the two datasets considered here (ELF-MF exposure and ionising radiation) (Figure 24), it is illustrated that no single gene was differentially expressed 12 weeks following a 15 hour, $300 \mu$ T ELF-MF exposure. It follows that such an expression is likely representative of background global levels of exposure. Comparatively, following exposure to $1 \mathrm{~Gy}$ X-rays, Figure 24 not only illustrates the remnants of a global exposure response in the same transcripts 12 weeks post irradiation, but the expression levels of five transcripts have deviated significantly from the linear relationship expressed by the other transcripts, corresponding to those transcripts whose expression levels were significantly up-regulated in Figure 22. Such a visual representation of the data therefore confirms the robustness of the previous SM-PCR analyses, and moreover, that the effects of ELF-MF on gene expression are negligible compared to those following exposure to $1 \mathrm{~Gy} \mathrm{X}$-rays. 


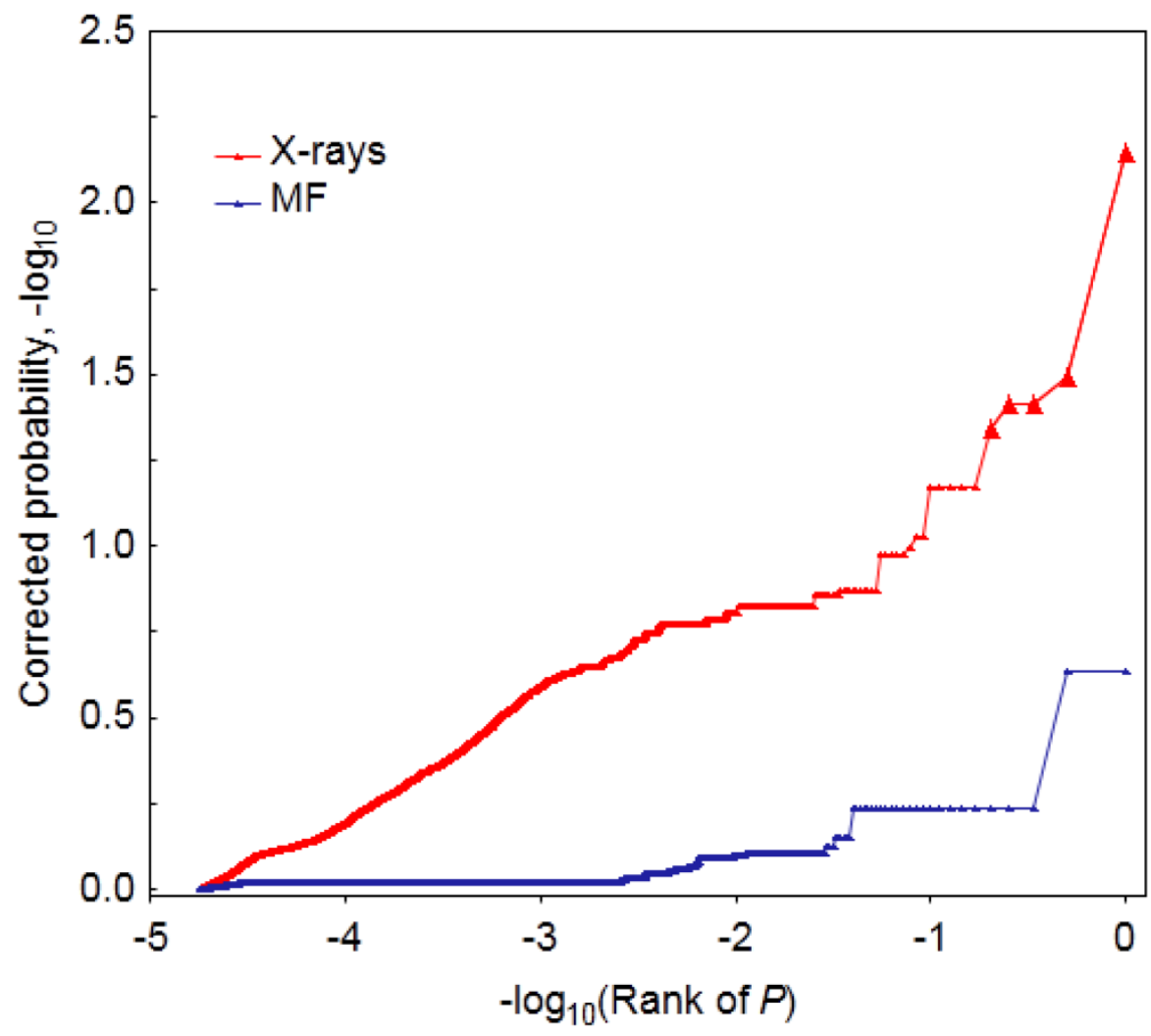

Figure 24. Probability plot analysis comparing the exposure distributions between ELF-MF and X-ray irradiated samples. The $P$-values are ranked and then logtransformed $\left(-\log _{10}\right)$ in order of rank ( $x$-axis). These are compared to the negative $\log _{10}$ transformed corrected $P$ values plotted on the $y$-axis.

\section{Discussion}

\subsection{Summary of Data}

In this thesis, the frequency of germline and somatic ESTR mutation induction was analysed following 2- or 15 hour exposures to $50 \mathrm{~Hz}$ magnetic fields of 10, 100 or 300 $\mu \mathrm{T}$. The data presented from the studies within this thesis demonstrate that in neither tissue does the frequency of ESTR mutation induction of all exposed male mice significantly differ from that in their equivalent sham-treated groups (Figures 16, 17). Moreover, a lack of significant difference in ESTR mutation frequency remained in both the germline and somatic tissues of exposed mice when compared to that of an aggregated group of sham-treated controls. In contrast, the frequency of ESTR mutation was significantly elevated in both the germline and somatic tissue of males exposed to $1 \mathrm{~Gy}$ of acute X-rays (Table 17). 
These data were in part confirmed by an additional pilot study in which highthroughput microarray analysis further demonstrated the lack of any significant alteration in gene expression in kidney cells of $\mathrm{BALB} / \mathrm{C} \times \mathrm{CBA} / \mathrm{Ca}$ hybrid $\mathrm{F}_{1}$ males following a 15 hour exposure to $50 \mathrm{~Hz}$ magnetic fields of $300 \mu \mathrm{T}$ (Figure 21).

Overall, these studies represent, to the best of my knowledge, the first methodical attempt to determine the in vivo frequency with which mutation induction occurs in the germline and somatic tissues of male mice after continuous exposure to $50 \mathrm{~Hz}$ ELFMFs up to a dose of $300 \mu \mathrm{T}$ (Wilson et al., 2015).

\subsection{Epidemiological Data}

Throughout this thesis, a constant emphasis has been placed on how exposure to ELFMF background environmental fields is unavoidable. The development of electrical technology and expansion of their use within modern society has led to an increase in both residential and occupational exposure to ELF-MFs. Owing to such continuous worldwide ELF-MF exposure, it is therefore important to analyse and understand the effects of ELF-MF on living organisms. As such, over the years, the effects of ELF-MF have been extensively studied in regards to both their impact on general health and at the DNA level, using an array of techniques, doses, measurement strategies and systems. Yet, this has resulted in a contradicting body of literature. Thus, an issue still remains as to the potential adverse biological/genotoxic effects of residential and/or occupational ELF-MF exposure. It was therefore owing to this discrepancy that the present study was designed in order to investigate the possible mutagenic potential of low dose ELF-MF exposure in vivo. This was achieved through the analysis of mutation induction frequency in both the somatic and germline tissues of $B A L B / C \times C B A / C a$ hybrid $F_{1}$ males, following exposure to 10,100 and $300 \mu$ T for 2 or 15 hours. These designated field strengths incorporate a spectrum of doses specifically chosen to encompass the International Commission on Non-Ionising Radiation's recommended exposure limits pertaining to the general public, which is set at $200 \mu \mathrm{T}$ for $50 \mathrm{~Hz}$ magnetic fields (International Commission on Non-Ionizing Radiation Protection, 2010). Moreover, they are inclusive of field strengths to which exposure has previously resulted in DNA damage in vitro (intermittent exposure, $\geq 35 \mu \mathrm{T}$, (Ivancsits et al., 2003b)). However, it is of note that these field strengths are substantially larger than 
those associated with the average household exposure in both the UK and USA (AGNIR, 2001), in addition to those reported in epidemiological studies associated with a diverse array of cancers, and in particular childhood leukaemia (IARC Working Group on the Evaluation of Carcinogenic Risks to Humans, 2002).

Indeed such data generated by epidemiological studies associating ELF-MF with an increased risk of childhood leukaemia are chiefly responsible for ELF-MFs group 2B classification as possibly carcinogenic (Chapter 1.12) (Ahlbom et al., 2000; Greenland et al., 2000; IARC Working Group on the Evaluation of Carcinogenic Risks to Humans, 2002). It is owing to such classification that the study of the health effects related to ELF-MF exposure continues to occur. Thus as epidemiological studies are the principal reference in classification, the evidence generated through these studies warrants further discussion.

\subsection{Epidemiological Limitations}

It is important to note that while the majority of epidemiological studies compensate for outside influences within their analyses, there is potential to attribute some causal role to that of possible confounding factors and/or selection bias in the designation of cases and/or controls. Yet, probably with the exception of traffic density, most studies sufficiently account for potential confounding factors and thus the impact upon the observed results from possible confounders is likely to be negligible (IARC Working Group on the Evaluation of Carcinogenic Risks to Humans, 2002). The same however is unlikely to be true for potential selection bias, as a review of the epidemiological literature by Mezei and Kheifets (2006) identified evidence to suggest it played a role both for and against an association between ELF-MF and childhood leukaemia.

One of the main problems with epidemiological studies is with the measurement of ELF-MF exposures. Since these investigations are almost exclusively conducted retrospectively, assessment of human health effects and in particular the study of chronic diseases in relation to ELF-MF exposure proves problematic since direct measurements of exposure during the aetiologically relevant period cannot be recreated. 
The early studies were based upon exposure estimates taken from wire codes rather than from measured values of direct personal exposures. The use of calculated field measurements using wire codes and distance has potential to counteract such fluctuations in exposure as the distance and wiring to residential areas remains stable over extended time periods. However, studies which rely on the use of calculated fields exclude all other sources of exposure which ultimately leads to an underestimation of exposure, while only a few report adequate exposure parameters. Moreover, the use of wire codes also has potential to introduce selection bias in terms of socioeconomic status in studies where controls were selected through random-digit dialling (Savitz et al., 1988; London et al., 1991; Linet et al., 1997). The existence of publication bias will further have an influence over the amount of data available for meta-analysis studies. However, these studies evaluated the influence of socioeconomic status on the observed results and each concluded that it was not a potential confounder.

In fact, the difficulties lie in the selection and recruitment of cases and controls since the average home is exposed to an average field strength of $<1 \mu \mathrm{T}$ (UK Childhood Cancer Study Investigators, 1999), with a fraction of homes with average fields above certain thresholds for example, $1 \%$ to $2 \%$ of homes in the United Kingdom and $10 \%$ in the United States presenting fields of $>0.2$ micro T (Kheifets et al., 2005). Therefore the designation of cases and controls at exposures above these limits proves difficult. Studies have consistently struggled to find sufficient cases to form a viable conclusion of effect, such as Dockerty et al. (1998), who identified suggestions of a positive association between CL and ELF-MF $\geq 0.2 \mu \mathrm{T}$ (Dockerty et al., 1998), although the study only possessed five cases and one control within this exposure group, and the confidence intervals produced were too wide to form a viable conclusion ( $\mathrm{Cl}$ : 1.1-224). Likewise in meta-analysis studies, as of the 12 studies included in the Greenland et al. (2000) study, only two (London et al., 1991; Linet et al., 1997) provided more than four cases exposed to $\geq 0.4 \mu \mathrm{T}$. Moreover, while it was highlighted by Ahlbom et al. (2000) that exposures $>0.4 \mu \mathrm{T}$ signify a doubling in risk of childhood leukaemia, it must also be noted that this exposure category was only representative of $0.8 \%$ of subjects ( 44 cases and 62 controls). 
As study design progressed, many studies still only invoked direct measurements such as spot measurements, despite the fact that these only assess exposure at one time point. Whilst this type of exposure assessment is able to offer a quantitative estimate of the field strength to which a household is exposed, such studies are supremely limited. Firstly, spot measurements exclusively measure background fields, failing to account for exposure metrics when using or within the vicinity of in use appliances. Secondly, exposure assessment of this kind is unrepresentative of long-term exposure to magnetic fields as it fails to account for the seasonal and long-term fluctuations in ELF-MF field strength. Taken together, these limitations suggest that the use of spot measurements in the retrospective assessment of ELF-MF exposure does not provide sufficient information regarding the aetiologically relevant period in order to sufficiently evaluate the association between exposure to ELF-MF and the increased incidence of childhood leukaemia. While 24-hour measurements represent an improvement over spot measurement, they too fail to account for fluctuations in field strength. Overall, direct measurement studies possess limitations in their evaluation of long-term exposure assessment, which may potentially lead to instances of exposure misclassification. It should however be noted that it is unlikely the subsequent association with disease status is due to the misclassifications of exposure. Indeed such misclassifications more prominently bias in favour of the null and are thus representative of an underestimation of the exposure effect (Rothman et al., 2008).

Evidence from epidemiological studies suggests that the presence of any potential association between childhood leukaemia and ELF-MF occurs through post-natal exposures of $>0.4 \mu \mathrm{T}$. Yet since epidemiological studies are limited in their analysis, scientific evidence is still lacking as to the effect of exposures greater than this. Moreover, whilst there have been positive epidemiological associations, uncertainties remain as to whether the association between ELF-MF and childhood leukaemia is causal. Yet, given how few cases and controls are actually exposed to ELF-MFs greater than $0.4 \mu \mathrm{T}$, it is roughly estimated that between one and two additional cases (per 500) would arise as a result of artificial ELF-exposure (UK Childhood Cancer Study Investigators, 1999; Kroll et al., 2010). Further analysis by Teepen and Dijck (2012) also 
estimated that ELF-MF exposure potentially only contributes an overall population attributable risk (PAR) percentage of 1.9(\%).

Taken together these statistical analyses indicate that exposure to ELF-MF is unlikely to significantly impact upon the incidence of childhood leukaemia if indeed causality is established. In light of the present findings, it is therefore posited that the classification of ELF-MF exposure as possibly carcinogenic represents a conservative and premature public-health decision. This decision has continued to drive an increasing number of studies to attempt to validate potential causality between ELFMF exposure and childhood leukaemia as indicated by epidemiological studies. However, such causality cannot be validated without establishing either a dosedependent response in vitro and in vivo or the existence of a plausible biophysical mechanism through which extremely low-frequency magnetic fields are proven to possess carcinogenic characteristics.

\subsection{Dose Response}

As such, this current study investigated the presence of a dose-dependent response to ELF-MF exposure. While analysis of pooled sperm data for each individual exposure length ( 2 hours or 15 hours) illustrated a marginally significant elevation in the total frequency of ESTR mutation when compared to that in sham-treated animals (Figure 18 , Table 19), these data do not however present any evidence to indicate a dosedependent increase within the exposed male mice. The lack of evidence with regards to an ELF-MF dose-response is seemingly in contrast to several earlier studies (Lai \& Singh, 1997; Ivancsits et al., 2002, 2003b; Wolf et al., 2005; Focke et al., 2010). However, all of these studies also provided evidence of ELF-MF exposure possessing genotoxic capabilities.

In the study by Lai and Singh (1997) brain cells of adult Sprague-Dawley rats were analysed 2 and 4 hours after acute ( 2 hours) exposure to a $60 \mathrm{~Hz}$ magnetic field at flux densities of $0.1,0.25$ and $0.5 \mathrm{mT}$. Using the previously employed micro-gel electrophoresis assay (Lai \& Singh, 1996), a significant dose-dependent increase in DNA single-strand breaks was observed at all flux densities in which the average DNA migration lengths increased in conjunction with flux density. A similar dose-dependent response was also witnessed in double-strand breaks, however only following 
exposure to 0.25 and $0.5 \mathrm{mT}$ (Lai \& Singh, 1997). Similar dose-responses were identified in ensuing studies, although these studies implemented intermittent exposures of ELF-MFs. Progressive intermittent ELF-MF exposures (20-1000 $\mu$ T, 5 min on/10 min off) of cultured human fibroblast cells for a period of 1 to 24 hours resulted in both a dose-dependent and time-dependent increase in DNA single- and doublestrand breaks as measured by alkaline and neutral comet tail factors (Ivancsits et al., 2003b). DNA damage increased in a time-dependent manner, although it peaked at 15 hours with the assay levels declining thereafter without returning to basal levels. Likewise, an increasing scale of magnetic flux densities from 20 to $1000 \mu \mathrm{T}$ induced a dose-dependent significant increase in single- and double-strand breaks from a magnetic flux density of $35 \mu \mathrm{T}$ (Ivancsits et al., 2003b). Additionally, Wolf et al. (2005) reported a dose-dependent increase in DNA damage, detected as DNA strand breaks and measured by the comet assay, in three separate cell lines (HL-60 leukaemia cells, Rat-1 fibroblasts and human diploid fibroblasts (WI-38 cells) exposed for 3-72 hours to 0.5-1.0 mT, $50 \mathrm{~Hz}$ ELF-MF, where genotoxicity peaked at 24 and 72 hours (Wolf et al., 2005). Finally, Focke and co-authors (2010) were able to replicate an ELF-MF exposure dependent increase in comet tail DNA in three cell models of primary human fibroblasts (ES-1, HR-1d and MRC-5) intermittently exposed to $50 \mathrm{~Hz}$ ELF-MF at a flux density of $1 \mathrm{mT}$. However, statistical significance was smaller and with higher experimental variation than those previously presented by Ivancsits et al. (2002).

Ultimately, since there are currently neither consistent laboratory evidence in support of a dose response, nor any viable biophysical mechanism through which extremely low-frequency magnetic fields can biologically interact with and give rise to mutations in mammalian tissues, the presence and shape of a dose response cannot be accurately ascertained. Moreover, without the foundation of dose-effect relationships, the validity of the causal effect of extremely low-frequency magnetic field irradiations on any increased risk of cancerous diseases, as portrayed by epidemiological studies, cannot be explained by any scientific reasoning. 


\subsection{Comparative Analysis of Study Results}

\subsubsection{ELF-MF Data}

It is not solely the presence of dose-dependent responses to ELF-MF exposure which is seemingly lacking conclusive supporting data; the absence of viable data replication, both between and within laboratories, has contributed to the contradictory nature of available data regarding this subject. While the majority of studies have been unable to confirm the genotoxic nature of magnetic field exposure, conclusions of such nature have been questioned with arguments pertaining to the lack of assay sensitivity in studies where an absence of ELF-MFs carcinogenic potential has been determined (Mairs et al., 2007). It follows that, while the classic methods provide the sensitivity capable of identifying extensive and severe cellular DNA damage, they may lack the relevant sensitivity required to reveal subtle molecular alterations that lead to potentially harmful genetic damage (Mairs et al., 2007). In light of this, previous studies have called for the implementation of sensitive high-throughput methods in the detection of ELF-MFs potential genotoxic effects (Burdak-Rothkamm et al., 2009). Indeed it has been illustrated throughout that the use of hypervariable tandem repeat loci located within the mammalian genome has been previously employed as a successful biomarker in the detection of induced germline mutations in humans and animals following environmental exposure to radioactive and chemical pollutants (Chapters 1.14 to 1.14.3.2.2) (reviewed in Yauk, (2004)). Therefore, by having recourse to ESTRs in the current study, a highly sensitive in vivo system was employed in order to assess any mutagenic effects of ELF-MFs at levels comparable to those populations are exposed to. The data presented through the implementation of this system are in line with the majority of previous findings in which ELF-MF exposure does not significantly alter the frequency of mutation induction in vivo.

Yet further comparison has proven difficult as there are a limited number of in vivo studies which have been conducted to date. Not only that but studies in which mutational analysis was conducted post-ELF-MF exposure are fewer still. Moreover, the majority of mutation analysis studies that have been performed have investigated the biological effects of ELF-MF at high flux densities (Miyakoshi et al., 1996, 1997; 
Mairs et al., 2007), and are thus neither representative of those flux densities employed within this study, nor of true environmental ELF-MF exposures.

A series of studies investigated the effect of ELF-MF (50 Hz at $400 \mathrm{mT}$ ) exposure on the induction of 6-thioguanine-resistant $\left(6-\mathrm{TG}^{\mathrm{r}}\right)$ mutations in the HPRT gene of cultured human melanoma (MeWo) cells (Miyakoshi et al., 1996, 1997). An increase in induced 6-thioguanine-resistant (6-TG') mutations was observed between 4- and 10 hours after a 2 hour exposure (Miyakoshi et al., 1997). Similarly, in a further study, the authors also reported that exposure to a $50 \mathrm{~Hz}$ magnetic field at $400 \mathrm{mT}$ for up to 20 hours induced a 6-fold increase in 6-thioguanine-resistant (6-TG $\left.{ }^{r}\right)$ mutations as compared to the sham-treated control (Miyakoshi et al., 1996). Furthermore, the frequency of mutations increased in a time-dependent manner until becoming, in their opinion, saturated after 10 hours (Miyakoshi et al., 1996). Conversely, an additional study in which a 12 hour exposure to a $60 \mathrm{~Hz}, 0.7 \mathrm{mT}$ magnetic field did not enhance the mutation frequency in Chinese hamster ovary cells exposed in the absence of ionising radiation (Walleczek et al., 1999); the latter findings are more in line with those from this present study.

However, it has been concluded that the analysis of alteration in hypervariable tandem repeat DNA sequences such as microsatellite and minisatellite may provide a radically enhanced analysis of mutation frequencies when compared to the HPRT locus (Ogheri et al., 1995). The use of these non-coding DNA sequences in identifying radiationinduced mutations has previously been shown to represent at least a 1000 times more sensitivity than analysis of the HPRT gene, which is renowned as the current benchmark against which other methods of DNA mutation detection are measured (Boyd et al., 2000). Indeed the use of hypervariable tandem repeats within this present study to analyse the mutagenic potential of ELF-MF is not a novel idea as a former study conducted by Mairs and co-authors (2007) previously used microsatellite DNA sequences. Mairs and co-authors, (2007) studied the in vitro effects of ELF-MF. UVW human glioma cells were exposed to a $1 \mathrm{mT}, 50 \mathrm{~Hz}$ magnetic field for 12 hours after which the analysis of mutation induction at microsatellite loci was conducted. DNA damage was determined through the analysis of three types of microsatellite sequence mutation inductions (change in allele size, loss of heterozygosity and allelic imbalance). 
The alteration in allele size directly correlates to the measurement of mutation induction conducted within this thesis, whereby any alteration was due to an addition or deletion of repeat units. The authors reported that exposure induced an additional 0.011 mutations/locus/cell when compared to the sham-exposed cells, equating to a 3.75-fold increase in mutation induction (Mairs et al., 2007).

Since the ESTR loci used within this project are also likely to represent a class of highly expanded microsatellites (Hardwick et al., 2009), the data presented by the current thesis' study, in which an absence of mutation induction was found after ELF-MF exposure, are in direct contrast to the data presented by Mairs et al. (2007). Though it should be noted that the field strength to which the glioma cell line were exposed was approximately three times higher than the largest employed within the present study.

However, the present study was also designed, in part, in comparison to those previously conducted via the REFLEX programme (Ivancsits et al., 2002, 2003b; Winker et al., 2005), in which exposure to intermittent and continuous magnetic field exposures resembling doses near the IARC environmental safe-guards resulted in an increase in DSBs. As has been mentioned, the data of this current study are in contrast to those previously presented by the REFLEX programme (Ivancsits et al., 2002, 2003b; Winker et al., 2005). Likewise are those of Burdak-Rothkamm et al. (2009), whose analysis of the in vitro effects of similar intermittent exposure doses (50-1000 $\mu \mathrm{T})$ failed to identify any measureable alterations in the amount of double-strand DNA breaks and sister chromatid exchanges. Despite the $\mathrm{pH} 2 \mathrm{AX}$ method employed in their study possessing the ability to reliably detect DNA damage equivalent to an X-ray dose of $0.025 \mathrm{~Gy}$, neither the alkaline comet assay, nor the $\mathrm{\gamma H} 2 \mathrm{AX}$ assay could detect significant damage at the DNA breakage level in the MF exposed fibroblasts and thus confirm the REFLEX programme's data. Similarly, a more recent in vivo study by Saha et al. (2014), in which similar exposure parameters to those implemented in this current study were utilised (continuous 2 hour exposure to $100 \mu$ T and 15 hours exposure to $300 \mu \mathrm{T}, 50 \mathrm{~Hz} \mathrm{MF}$ ) and failed to provide any evidence of mutagenesis following $50 \mathrm{~Hz}$ magnetic field exposure. In the study conducted by Saha et al. (2014), 53BP1 foci were used as a direct signalling response to the formation of DSBs. The use of 53BP1 foci represents a sensitive monitor of DSB formation and repair, with nearly 
$100 \%$ efficiency. Pregnant C57BL/6 mice were exposed to a continuous $50 \mathrm{~Hz}$ magnetic field of $100 \mu$ T for 2 hours or to a continuous or an intermittent ( $5 \mathrm{~min}$ on/10 min off) field of $300 \mu$ T for 15 hours. Through this process, Saha et al. (2014) were able to provide further evidence that continuous exposures to either a 2 hour, $100 \mu$ T, or 15 hour, $300 \mu \mathrm{T}, 50 \mathrm{~Hz}$ magnetic field does not cause direct DNA damage.

Therefore these data, while using a different detection method, implement similar exposure conditions in vivo to this present study and further provide no evidence that the frequency in which mutations are induced is affected following exposure to $50 \mathrm{~Hz}$ magnetic fields up to a dose of $300 \mu \mathrm{T}$, ultimately strengthening these findings.

Moreover, data from previous ESTR studies have provided an ESTR mutation rate with a detection sensitivity cut-off equivalent to an exposure to $0.5 \mathrm{~Gy}$ of acute $\mathrm{X}$ - $/ \mathrm{Y}$-rays (Dubrova et al., 1998a; Mughal et al., 2012). Consequently, to findings herein, it is posited that the mutagenic effects on the public of MF exposure are only likely to cause ESTR mutations equivalent to 0.5 Gy of X-rays, if any at all. In fact, the study conducted by Saha et al. (2014) provided evidence of increased assay detection sensitivity equivalent to $10 \mathrm{mGy} X$-rays, therefore suggesting a lower threshold.

What is abundantly clear from reviewing all the literature in this area is that any ELFMF induced biological effects on DNA damage or gene expression, are subject to a range of factors and characteristics including, but not limited to, the frequency of emittance and the intensity, duration and mode of said exposure (Ivancsits et al., 2002). However, contradictory results regarding genotoxic potential of extremely lowfrequency magnetic fields are common within the literature; this may be due to the differences in cellular targets examined by individual studies. Ivancsits et al. (2005) reported that six types of cultured cells derived from different tissues induced different genotoxic responses when exposed to $1 \mathrm{mT}$ intermittent ELF-MFs. Of the six, there were three responder cell types consisting of: human fibroblasts, human melanocytes and rat granulosa cells, and three non-responder types: human lymphocytes, human monocytes and human skeletal muscle cells. Additionally, Luceri et al. (2005) failed to observe an increase in DNA damage in human blood lymphocytes exposed to $50 \mathrm{~Hz}$ magnetic fields at 1,10 or $100 \mu \mathrm{T}$ for 18 hours. 
These contradictory data are the product of exposure to several different cell lines and organisms which evoked different responses. These discrepancies occur since most of these aforementioned studies employ transformed cells known to exhibit inherent genetic instability. Similarly, primary cell cultures may prove unreliable since, when reaching maturation, they may present high background damage. Therefore the uses of in vivo analyses and of similar exposure parameters, both in the present study and within the Saha et al. (2014) study, overcome these limitations and ultimately start to provide a clear and concise conclusion regarding the effects of ELF-MF on biological systems which were previously unavailable.

\subsubsection{Ionising Radiation}

Within this current study, the use of $1 \mathrm{~Gy}$ of acute X-rays was applied as a positive control in order to validate the SM-PCR technique. Additionally, the analysis of ESTR mutation induction has, to date, predominantly been studied in the germline of male mice following exposure to ionising radiation (Dubrova et al., 1993, 1998a, 1998b, 2000b; Sadamoto et al., 1994; Fan et al., 1995; Niwa et al., 1996; Dubrova \& Plumb, 2002; Yauk et al., 2002; Dubrova, 2005). However, such effects in somatic tissues are yet to be sufficiently established. Initial data correspond to similar mechanisms, as in the germline, whereby the rate of mutation induction was elevated in stem cells 10 and 20 weeks post-irradiation, in a stage-specific manner (Dubrova et al., 1998a, 1998b). Moreover, relatively small changes in mutation rates were exhibited in bone marrow cells following acute exposure to X-rays, while no discernible change in mutation frequency appeared in non-proliferating adult brains (Barber et al., 2009). Since the mitotic proliferation capabilities in an adult brain are low to non-existent (Gross, 2000), with brain cells only undergoing mitotic proliferation in utero, these data further signify that ESTR mutations arise almost entirely in replication-proficient cells (Barber et al., 2009).

Similarly, data from numerous studies analysing the ESTR mutation rate following acute irradiation to X-rays at various stages of spermatogenesis have previously shown that all radiation-induced ESTR mutations tend to arise at every stage of spermatogenesis preceding metaphase I (Barber et al., 2000). That is, mutation rates are significantly elevated only in spermatogonia derived from mid and early 
pachytene, type B spermatogonia and $A_{s}$ spermatogonia (Dubrova et al., 1998b; Barber et al., 2000), which are indicative of samples taken from offspring conceived 4, 5, or 6 and above weeks post-irradiation, representing pre-meiotic spermatogonia (Searle, 1974). In contrast, no measurable alterations in the frequency of ESTR mutations were exhibited in post-meiotic spermatids procured from non-dividing sperm cells of irradiated adult mice analysed either one week (Barber et al., 2009) or three weeks (Dubrova et al., 1998b; Barber et al., 2000) after exposure.

Since hematopoietic tissues in mice undergo a vast turnover (Metcalf, 1988), the design of this current study, in which nucleated blood cells were sampled 12 weeks post-exposure, ensured that the majority of sampled nucleated blood cells originated from exposed replication-proficient stem cells. As indicated by Table 17, exposure to 1 Gy of acute X-rays causes a statistically significant increase in both the germline and somatic tissues of irradiated male mice. Yet, while the spontaneous mutation frequencies remain relatively similar in both blood and sperm $(t=1.23 ; P=0.2188)$, the radiation-induced ESTR mutation frequency in sperm is significantly higher than that in blood ( $t=2.45 ; P=0.0144)$, (Figure 18). A statistically significant 2.4-fold increase was shown in the germline ESTR mutation frequency of irradiated males, while a significant though less distinct 1.8-fold increase was reported in the blood of these same male mice.

The reasoning for such a less-pronounced increase in ESTR mutation induction in the hematopoietic stem cells, compared to the spermatogonial cells, is as yet unknown and should warrant further analysis in the future. A potential rationale however may be related to the cellular replication proficiency of the two tissues, such that the percentage of actively dividing hematopoietic stem cells in mouse bone marrow is very low ( 9.1/10 ${ }^{5}$ cells in BALB/c mice) (Muller-Sieburg \& Riblet, 1996), when compared to that of sperm.

Within the context of the analysis of ESTR mutation induction in the somatic tissues of male mice exposed to ionising radiation, the current findings represent the initial analysis of ESTR mutation induction in blood cells of male mice exposed to ionising radiation. Moreover, these findings contribute further support to the original 
hypothesis whereby mutations arising in germline or somatic tissues, either spontaneously or induced, do so through a similar mutation process (Hardwick et al., 2009).

\subsection{Pilot Study Microarray Summary}

In addition to the evaluation of the rate at which mutations are induced following ELFMF exposure, the present study also initiated a pilot study in which high-throughput microarray analysis was used to assess the pattern of gene expression in vivo following exposure to a $50 \mathrm{~Hz}$ magnetic field $(300 \mu \mathrm{T})$ for 15 hours. This preliminary study implemented a one-colour microarray-based gene expression analysis, whereby the highly sensitive Agilent SurePrint G3 Mouse GE 8x60K microarrays were used in the analysis of kidney samples taken from five $B A L B / c \times C B A / C a F_{1}$ hybrid male mice following a 15 hour exposure to a $50 \mathrm{~Hz}$ magnetic field of $300 \mu \mathrm{T}$. The primary data did not reveal any differentially expressed loci following exposure to $300 \mu \mathrm{T}$ for 15 hours compared with their sham-treated controls (Figure 21).

\subsubsection{Comparative Analysis of Microarray Data}

Several hypotheses have been proposed regarding potential mechanisms through which ELF-MF ultimately interacts with biological systems, including the production of free-radicals (Chapter 1.13.2, reviewed in Simko \& Mattsson, (2004)). One proposal by Phillips (1993) referred to an ELF-MF mediated alteration in gene expression. Following this proposal, several studies performed small-scale analysis studies on a small proportion of various genes with a potential to be affected. Loberg et al. (2000) performed a small-scale analysis of the transcript levels of 588 cancer-related genes in $\mathrm{HL}-60$ and mammary epithelial cells after exposure to a $60 \mathrm{~Hz}$ magnetic field of $10 \mu \mathrm{T}$ or $1 \mathrm{mT}$ for 24 hours. Likewise, Coulton et al. (2004) analysed the pattern of gene expression in HSP27, HSP70A or HSP70B of human leukocytes. Neither study reported significant alterations in the expression profiles in any of the 588 cancer-related genes (Loberg et al., 2000), nor that $50 \mathrm{~Hz}$ magnetic fields up to $100 \mu \mathrm{T}$ altered the pattern of gene expression in HSP27, HSP70A or HSP70B of human leukocytes (Coulton et al., 2004).

However, the effect of human exposure on health appears to be multifaceted. While the absence of biological mechanism and failure to establish a dose-response means 
that there is a lack of starting point at which to initiate investigations. Therefore, the use of whole-genome screening methods, like microarray gene expression analyses possess the potential to globally analyse genes, and could identify viable gene candidates which respond to ELF-MF exposure. The issue is that, similar to mutation analyses studies, there remains as yet a limited number of large-scale gene expression analyses evaluating the biological effects of ELF-MF exposure. The present pilot study invokes the use of a genome-wide platform of investigation in an attempt to gather preliminary data as to whether $50 \mathrm{~Hz}$ magnetic fields are able to interact with biological systems in vivo.

Previous attempts have been made to study the global gene-expression effects of ELFMF, although these have occurred in vitro. Luceri et al. (2005) assessed the variation in overall gene expression profiles in human lymphocytes cells and Saccharomyces cerevisiae after exposure to 1, 10 or $100 \mu \mathrm{T}$ for 18 hours. By using DNA microarray in the holistic analysis of the yeast and human genomes, an exposure to ELF-MFs ranging from 1-100 $\mu$ T for a period of 18 hours was not seen to render a change in any gene expression profiles. Although the yeast genome was analysed in full, the study of the human genome was limited in its design with only 13971 oligonucleotides represented on the array (Luceri et al., 2005). More recently, a study by Henderson et al. (2006) employed the use of microarrays containing approximately thirty thousand oligonucleotides in their analysis of the transcriptional response in human primary vascular endothelial cells. Neither continuous exposure of a $50 \mathrm{~Hz}$ MF representative of occupational $(700 \mu \mathrm{T})$ and domestic $(10 \mu \mathrm{T})$ environments for a period of 24 hours, nor intermittent exposure (700 $\mu \mathrm{T}, 60 \mathrm{~min}$ on/30 min off) over a 24 hour cycle gave rise to an effect on the expression of any genes analysed which could be replicated.

Similarly, Chen et al. (2012) analysed the gene expression response in Saccharomyces cerevisiae exposed to a $50 \mathrm{~Hz}$ magnetic field of $0.4 \mathrm{mT}$ for 6 hours, the use of which was attempting to mimic the 1998 ICNIRP occupational exposure guideline. Despite three genes being discovered to be up-regulated by at least 1.3 fold, upon further analysis with real-time reverse transcription-polymerase chain reaction (RT-PCR), the microarray detected changes were unconfirmed. After six independent replications real-time RT-PCR analysis failed to confirm any significant change in any of the three 
gene expression levels, with all three gene candidates presenting fold changes of approximately 1.0 .

While these three studies were performed in vitro, the exposure doses to which these cells were subject to are approximately equivalent to the one used in the present study (300 $\mu \mathrm{T}$ for 15 hours), and thus the preliminary data generated from this pilot study are in conjunction with those previously conducted (Luceri et al., 2005, Henderson et al., 2006; Chen et al., 2012).

Taken together, the data from these separately conducted global analysis studies on gene expression profiles suggest that $50 \mathrm{~Hz}$ magnetic fields of up to $400 \mu \mathrm{T}$ do not significantly alter the pattern of gene expression profiles in human lymphocytes, yeast cells or in vivo. Moreover, additional evidence from the study by Loberg et al. (2000) confirms that no significant changes were identified in the expression of genes related to the development of cancer. The results of this present study are in conjunction with, and further add to, the growing body of evidence which suggests that there is no causality between changes in gene expression and exposure to $50 \mathrm{~Hz}$ magnetic fields, nor do such magnetic fields possess any ability to interact with biological systems.

\subsection{Study Limitations}

The analysis of continuous exposure is viable as it allows the prolonged exposure to ELF-MF experienced by households inhabiting in close proximity to high-voltage power lines to be mimicked. However, only $1.1 \%$ of homes in the USA reside within 40 meters of high-voltage power lines, while in the UK this figure is further reduced to $0.07 \%$ of homes situated within 50 metres (EMFs.info, 2015). Moreover, whilst we are continually exposed to ELF-MF throughout domestic life, there are fluctuations in intensity dependent upon our location, the time of day or the season of the year. Whilst subsequent studies have not confirmed such findings (Burdak-Rothkamm et al., 2009; Saha et al., 2014), it was previously demonstrated that exposure to intermittent magnetic fields are not continuous, and result in a significant induction of DSB formation (Ivancsits et al., 2002, 2003b; Winker et al., 2005). Therefore, it is probable that the use of intermittent fields similar to those employed in previous studies (Ivancsits et al., 2002, 2003a, 2003b, 2005; Winker et al., 2005; Burdak-Rothkamm et al., 2009; Saha et al., 2014), would not only have allowed for a more realistic 
mimicking of global exposure but would have allowed data between studies to be more accurately compared.

Finally, while the use of non-coding repeat sequences currently represents the best laboratory model for studying mutagen-induced tandem repeat instability in the germline, this is mostly due to a lack of a more biologically-relevant model. Whilst the mouse genome does encompass the characteristic true minisatellite loci, the germline mutation rates exhibited by these loci $\left(<10^{-3}\right.$ per generation) cannot be considered hypervariable (Bois et al., 1998a). As such, to date there is no animal model in which the mutation induction at minisatellites can be appropriately studied, nor is there any such equivalent for a human ESTR model at this time. The issue lies in the fact that the mutational mechanism through which ESTR loci are processed is fundamentally different to that of human minisatellites (Kelly et al., 1989; Gibbs et al., 1993; Bois et al., 1998b). The high frequency germline mutations exhibited by human minisatellites arise through complex meiotic recombination events, whilst ESTR instability arises through a replication or repair-based process involving DNA polymerase slippage during DNA replication, or through DNA polymerase pausing to repair the DNA following the formation of secondary structures (Barber et al., 2004; Hardwick et al., 2009). Moreover, human minisatellites do not exhibit the same levels of somatic instability as ESTR loci (Jeffreys et al., 1988, 1994). This may cast doubts over the biological relevance of ESTR assays and their substitution for human minisatellite instability (reviewed in (Bouffler et al., 2006; Singer \& Yauk, 2010; Yauk et al., 2015)). However, the dose-response of ESTR mutation induction has been shown to correlate to that of other classical mutation scoring assays (Dubrova et al., 1998a; Barber et al., 2006). Additionally, not only is there an increasing amount of evidence of tandem repeat loci partaking an essential role in genome structure and function, but also evidence of an association with genes and location in and/or near promoter sites (Armour, 2006; Mirkin, 2007). This equates to an expanding amount of evidence associating mutations at tandem repeat loci with that of human genetic diseases, some of which even causally (Mirkin, 2007).

However, despite these findings, it would be premature to view these data as anything more than comparative until such a time where either a more biologically relevant 
animal model for minisatellite studies is produced, or until the presence of human ESTRs are confirmed and revealed to respond in the same biological manner to mutagen exposure.

\subsection{Conclusion of Findings}

In summary, this present study represents, to the best of my knowledge, the first methodical attempt to determine the in vivo frequency at which mutation induction occurs in the germline and somatic tissues of male mice after continuous exposure to $50 \mathrm{~Hz}$ ELF-MFs of doses up $300 \mu \mathrm{T}$ (Wilson et al., 2015).

This study was established in response to the repeatedly inconsistent data and absence of any independent replication which have frequented this area of scientific investigation. Thus, through the implementation of a highly sensitive biomarker within this study which has previously been applied with success in the detection of mutation induction within proliferating mouse tissues, no significant increases in the frequency of ESTR mutation in blood were detected following exposure to $50 \mathrm{~Hz}$ magnetic fields. Additionally, whilst a marginally significant increase was observed in the frequency of induced germline mutations, these data should be regarded cautiously due to the lack of an evident correlating dose response. Yet, it must be stressed that whilst causality could be inferred from a simultaneous increase of both disease risk and exposure dose, an absence of a dose response does not de facto indicate the lack of a causal relationship.

However, considering these findings and the absence of any significant alteration in gene expression profiles in mouse kidney cells after a continuous 15 hour exposure to $300 \mu \mathrm{T}$, it can be concluded that where individuals have experienced mutagenic effects as a result of ELF-MF exposure, then such effects equate to that of less than $1 \mathrm{~Gy} X$ rays in germline cells. Moreover, it can also be posited that such mutagenic effects arising from exposure observed in somatic tissues are likely to be negligible (Wilson et al., 2015).

Nonetheless, while these findings will lend themselves to the assumption that observation of any of the adverse health effects stated within the literature are unlikely, it is too early to disregard the possibility of such an association because of the 
complex and diverse nature in the causation of human disease, as illustrated by the onset of childhood leukaemia. As such, it follows that the biological effects of ELF-MF and any link it may have to adverse health effects should be approached cautiously as lacking scientific validation. This should remain so until either the detection of a persistent biophysical mechanism, or an irrefutable biological response is witnessed consistently within independent laboratories in response to ELF-MF exposure. Yet until such a time, it remains that such adverse health effects will continue to be considered with uncertainty by certain groupings within the scientific community.

\subsection{Future Work}

\subsubsection{Microarray Analysis}

Since ELF-MF is representative of such a weak stimulus, the additional approach of global gene expression analysis could potentially identify responding gene candidates and ultimately determine a potential biological mechanism. While microarray analyses are capable of detecting changes in expression equivalent to a two-fold or greater increase, they are limited in terms of detecting minor changes.

Therefore, a further study would aim to continue the gene expression analysis and implement the use of a more powerful method, such as RNA-sequencing (RNA-Seq) (Wang et al., 2009, Fonseca et al., 2014), to initially confirm that the lack of microarray detected gene alterations in response to ELF-MF exposure are indeed accurate and not in fact false negatives. RNA-Seq represents a more powerful alternative to transcriptome profiling than microarrays, utilising next generation sequencing technology to provide a high-throughput and quantitative analysis, through sequencing the whole transcriptome at the single-nucleotide level.

The implementation of such a sequencing based method to directly determine the CDNA sequence allows the investigation of mRNAs, small RNAs and non-coding RNAs expression levels, and provides a more precise measurement and discovery of novel and rare transcripts alongside their splice isoforms. Ultimately, the use of RNA-Seq allows for the detection of global gene expression patterns that were previously inaccessible using hybridisation-based methods such as microarrays. Indeed, unlike hybridisation-based approaches, RNA-Seq allows the detection of transcripts beyond 
those corresponding to existing genomic sequences. This particular property makes RNA-Seq a seemingly attractive analysis tool for non-model organisms with genomic sequences that are yet to be determined. Additionally, in contrast to microarray methods, RNA-Seq presents minimal to no background and signal saturation, thereby providing a higher sensitivity for genes expressed either at low or very high levels. It follows that RNA-Seq presents a higher dynamic range than that of DNA microarrays (one hundred fold to a few hundred fold) (Wang et al., 2009). Finally, there is no amplification step allowing RNA-Seq analysis to be performed using less RNA

A comparative study of genome-wide gene expression analysis using the same RNA samples with a RNA-Seq and Agilent $4 \times 44 \mathrm{~K}$ oligonucleotide microarray platforms validated the performance metrics of both assays using quantitative real-time PCR discovered that RNA-Seq outperformed Agilent in all categories: sensitivity (78\% vs $51 \%)$, specificity ( $75 \%$ vs $38 \%$ ), precision ( $76 \%$ vs $46 \%)$, accuracy $(77 \%$ vs $44 \%$ ) and false discovery rate ( $24 \%$ vs $54 \%$ ) (Nault et al., 2015). A further study similarly measured expression levels for 48 genes using RNA-Seq, microarray and real-time PCR methods. In accordance with Nault et al. (2015), Li and co-authors (2016) discovered a consistent level of expression between RNA-Seq and real-time PCR for 40 of the 48 genes (83.3\%). Yet conversely, only 18 of the 48 genes (37.5\%) were observed to demonstrate consistent expression levels when comparing microarray and real-time PCR approaches (Li et al., 2016). The use of RNA-Seq therefore provides a beneficial accompaniment to the microarray-based gene expression analysis. Similarly, the use of RNA-Seq should also provide new insights into the effects of exposure to ionising radiation on gene expression.

\subsubsection{Mutational Analysis}

Although in this present study it has been demonstrated that the frequencies of ESTR mutation in germline and somatic tissues of male mice exposed for 15 hours do not exceed those following a shorter 2 hour exposure, further analysis of the genotoxic effects of long-term exposure to magnetic fields would prove informative. Indeed, a recent in vivo study analysed the long-term (28 day) exposure to $200 \mu \mathrm{T}$ of $50 \mathrm{~Hz}$ magnetic field, finding that the yield of micronucleated polychromatic erythrocytes were significantly increased in mice (Alcaraz et al., 2014). Such findings are further 
supported by other previous publications (reviewed in Udroiu et al., (2010)), and seem to indicate that ELF-MFs only induce noticeable genotoxic effects after long-term exposure. Therefore future work could involve the in vivo examination of mice exposed for a longer period than the 15 hours implemented in this present study. The use of the same highly sensitive ESTR loci biomarker in addition to extended exposure duration would ultimately provide information as to the risk of ELF-MF exposure over a lifetime.

The use of intermittent exposure parameters is more representative of worldwide exposure, as one's exposure to ELF-MF is constantly fluctuating, with the field undergoing change depending on the varying use of household appliances. Also, some experimental studies in which a genotoxic effect was observed occurred after exposure to intermittent ELF-MF exposure as opposed to continuous exposure. Whilst this has not been confirmed by others (Burdak-Rothkamm et al., 2009; Saha et al., 2014), the use of ESTR loci in the analysis of intermittent ELF-MF will provide the foundations to unequivocally conclude either finding. It follows that future studies should be designed as a way of validating whether alterations in gene expression response, alongside any induced DNA damage, are dependent on either continuous or intermittent exposure. Furthermore, if indeed the genotoxic effect is determined by such exposure parameters, then an explanation will need to be determined as to why intermittent and not continuous exposures should induce such effects.

Additionally, while there are currently numerous hypotheses relating to the mechanism through which ELF-MF may possess genotoxic and carcinogenic potential, the radical pair mechanism remains to date the most feasible method through which environmentally weak magnetic fields could potentially interact with mammalian biochemical systems. Yet, it follows that it is a fallacy to rationalise that biological systems are so fragile that any minor alteration in the homeostasis of intracellular radical concentration arising from interaction with weak magnetic fields could have a genotoxic effect. One exception would arise where ELF-MF exposure was administered in the presence of an amplification agent or the exposed system exhibited an already weakened defence system. While this current study was not designed to further the available data or add to the understanding of any mechanism involved with ELF-MF 
exposure in itself, the widespread presence and functions of radicals within biological systems coupled to the presence of conflicting data within the literature, make it abundantly clear that further experimental and theoretical work is needed in this field. Such future studies should ultimately be performed in vivo in order to fine-tune the conditions relating to ELF-MF sensitivity. These future analyses could finally determine whether exposure to ELF-MFs, within the range of doses used in the current study, can alter the balance of melatonin and free radicals, since as of yet, no appropriate comparison can be made.

A final point should be made that there is a need for more in vitro studies which analyse the effects on human cells, not only to perpetuate the investigation into potential health risks, but also to better understand the occurrence of organism mutations and their biological interactions with ELF-MF exposure. Of particular note, the potential genotoxic role of free radicals and their relationship with the cell during and after exposure are yet to be clarified. Ultimately, in a field where the amount of in vivo studies are minimal, the development of a novel mouse model representative of childhood ALL presents an ideal opportunity to explore any effects induced by environmental exposures and the mechanics of any such occurrences (Li et al., 2013). 


\section{Reference List}

Abel, E.L., Hendrix, S.L., McNeeley, G.S., O'Leary, E.S., Mossavar-Rahmani, Y., Johnson, S.R., Kruger, M., 2007. Use of electric blankets and association with prevalence of endometrial cancer. European Journal of Cancer Prevention : The Official Journal of the European Cancer Prevention Organisation (ECP). 16, 243-250.

Abouzeid Ali, H.E., Barber, R.C., Dubrova, Y.E., 2012. The effects of maternal irradiation during adulthood on mutation induction and transgenerational instability in mice. Mutation Research. 732, 21-25.

(AGNIR) Advisory Group on Non-ionising Radiation. 2001. ELF Electromagnetic Fields and the Risk of Cancer. Report of an Advisory Group on Non-ionising Radiation. Documents of the NRPB 12 (1). pp. 5-179.

Ahlbom, A., 2001. Neurodegenerative diseases, suicide and depressive symptoms in relation to EMF. Bioelectromagnetics. Suppl 5, S132-43.

Ahlbom, A., 1988. A review of the epidemiologic literature on magnetic fields and cancer. Scandinavian Journal of Work, Environment \& Health. 14, 337-343.

Ahlbom, A., Day, N., Feychting, M., Roman, E., Skinner, J., Dockerty, J., Linet, M., McBride, M., Michaelis, J., Olsen, J.H., Tynes, T., Verkasalo, P.K., 2000. A pooled analysis of magnetic fields and childhood leukaemia. British Journal of Cancer. 83, 692698.

Ahlbom, A., Feychting, M., Gustavsson, A., Hallqvist, J., Johansen, C., Kheifets, L., Olsen, J.H., Medicinska och farmaceutiska vetenskapsområdet, Uppsala universitet, 2004. Occupational Magnetic Field Exposure and Myocardial Infarction Incidence.

Epidemiology. 15, 403-408.

Ahlbom, I.C., Cardis, E., Green, A., Linet, M., Savitz, D., Swerdlow, A., ICNIRP (International Commission for Non-lonizing Radiation Protection) Standing Committee on Epidemiology, 2001. Review of the epidemiologic literature on EMF and Health. Environmental Health Perspectives. 109 Suppl 6, 911-933.

Akdag, M.Z., Dasdag, S., Ulukaya, E., Uzunlar, A.K., Kurt, M.A., Taskin, A., 2010. Effects of extremely low-frequency magnetic field on caspase activities and oxidative stress values in rat brain. Biological Trace Element Research. 138, 238-249.

Akdag, M.Z., Dasdag, S., Uzunlar, A.K., Ulukaya, E., Oral, A.Y., Celik, N., Aksen, F., 2013. Can safe and long-term exposure to extremely low frequency $(50 \mathrm{~Hz})$ magnetic fields affect apoptosis, reproduction, and oxidative stress? International Journal of Radiation Biology. 89, 1053-1060. 
Alcaraz, M., Olmos, E., Alcaraz-Saura, M., Achel, D.G., Castillo, J., 2014. Effect of longterm $50 \mathrm{~Hz}$ magnetic field exposure on the micronucleated polychromatic erythrocytes of mice. Electromagnetic Biology and Medicine. 33, 51-57.

Allegra, M., Reiter, R.J., Tan, D.X., Gentile, C., Tesoriere, L., Livrea, M.A., 2003. The chemistry of melatonin's interaction with reactive species. Journal of Pineal Research. 34, 1-10.

Andersen, T.H. \& Nilsson-Tillgren, T., 1997. A fungal minisatellite. Nature. 386, 771.

Anderson, L.E., Morris, J.E., Miller, D.L., Rafferty, C.N., Ebi, K.L., Sasser, L.B., 2001. Large granular lymphocytic (LGL) leukemia in rats exposed to intermittent $60 \mathrm{~Hz}$ magnetic fields. Bioelectromagnetics. 22, 185-193.

Angelillo, I.F. \& Villari, P., 1999. Residential exposure to electromagnetic fields and childhood leukaemia: a meta-analysis. Bulletin of the World Health Organization. 77, 906-915.

Antonopoulos, A., Yang, B., Stamm, A., Heller, W.-., Obe, G., 1995. Cytological effects of $50 \mathrm{~Hz}$ electromagnetic fields on human lymphocytes in vitro. Mutation Research Letters. 346, 151-157.

Armour, J.A., 2006. Tandemly repeated DNA: why should anyone care? Mutation Research. 598, 6-14.

Banks, R.S., Thomas, W., Mandel, J.S., Kaune, W.T., Wacholder, S., Tarone, R.E., Linet, M.S., 2002. Temporal trends and misclassification in residential $60 \mathrm{~Hz}$ magnetic field measurements. Bioelectromagnetics. 23, 196-205.

Barber, R., Plumb, M., Smith, A.G., Cesar, C.E., Boulton, E., Jeffreys, A.J., Dubrova, Y.E., 2000. No correlation between germline mutation at repeat DNA and meiotic crossover in male mice exposed to X-rays or cisplatin. Mutation Research. 457, 79-91.

Barber, R., Plumb, M.A., Boulton, E., Roux, I., Dubrova, Y.E., 2002. Elevated mutation rates in the germ line of first- and second-generation offspring of irradiated male mice. Proceedings of the National Academy of Sciences of the United States of America. 99, 6877-6882.

Barber, R.C., Hardwick, R.J., Shanks, M.E., Glen, C.D., Mughal, S.K., Voutounou, M., Dubrova, Y.E., 2009. The effects of in utero irradiation on mutation induction and transgenerational instability in mice. Mutation Research. 664, 6-12.

Barber, R.C., Hickenbotham, P., Hatch, T., Kelly, D., Topchiy, N., Almeida, G.M., Jones, G.D., Johnson, G.E., Parry, J.M., Rothkamm, K., Dubrova, Y.E., 2006. Radiation-induced transgenerational alterations in genome stability and DNA damage. Oncogene. 25, 7336-7342. 
Barber, R.C., Miccoli, L., van Buul, P.P., Burr, K.L., van Duyn-Goedhart, A., Angulo, J.F., Dubrova, Y.E., 2004. Germline mutation rates at tandem repeat loci in DNA-repair deficient mice. Mutation Research. 554, 287-295.

Barnes, F.S. \& Greenebaum, B., 2007. Bioengineering and biophysical aspects of electromagnetic fields. [e-book]. 3rd ed. Boca Raton, Fla; London: CRC Press.

Belson, M., Kingsley, B., Holmes, A., 2007. Risk Factors for Acute Leukemia in Children: A Review. Environmental Health Perspectives. 115, 138-145.

Berne, R.M., Levy, M.N., Stanton, B.A. and Koeppen, B.M., 2005. Berne and Levy principles of physiology. 4th ed. Philadelphia, Pa: Elsevier Mosby.

Bois, P. \& Jeffreys, A.J., 1999. Minisatellite instability and germline mutation. Cellular and Molecular Life Sciences : CMLS. 55, 1636-1648.

Bois, P., Stead, J.D., Bakshi, S., Williamson, J., Neumann, R., Moghadaszadeh, B., Jeffreys, A.J., 1998a. Isolation and characterization of mouse minisatellites. Genomics. 50, 317-330.

Bois, P., Williamson, J., Brown, J., Dubrova, Y.E., Jeffreys, A.J., 1998b. A novel unstable mouse VNTR family expanded from SINE B1 elements. Genomics. 49, 122-128.

Bois, P.R., 2003. Hypermutable minisatellites, a human affair? Genomics. 81, 349-355.

Bois, P.R., Southgate, L., Jeffreys, A.J., 2001. Length of uninterrupted repeats determines instability at the unstable mouse expanded simple tandem repeat family MMS10 derived from independent SINE B1 elements. Mammalian Genome : Official Journal of the International Mammalian Genome Society. 12, 104-111.

Bonnell, J.A., 1982. Effects of electric fields near power-transmission plant. Journal of the Royal Society of Medicine. 75, 933-941.

Boorman, G.A., McCormick, D.L., Findlay, J.C., Hailey, J.R., Gauger, J.R., Johnson, T.R., Kovatch, R.M., Sills, R.C., Haseman, J.K., 1999. Chronic toxicity/oncogenicity evaluation of $60 \mathrm{~Hz}$ (power frequency) magnetic fields in F344/N rats. Toxicologic Pathology. 27, 267-278.

Boorman, G.A., Rafferty, C.N., Ward, J.M., Sills, R.C., 2000. Leukemia and lymphoma incidence in rodents exposed to low-frequency magnetic fields. Radiation Research. 153, 627-636.

Bouffler, S.D., Bridges, B.A., Cooper, D.N., Dubrova, Y., McMillan, T.J., Thacker, J., Wright, E.G., Waters, R., 2006. Assessing radiation-associated mutational risk to the germline: repetitive DNA sequences as mutational targets and biomarkers. Radiation Research. 165, 249-268. 
Boyd, A. Livingstone, L. E. Wilson, E. M. Marshall, A. G. McCluskey, R. J.

Mairs,T.E.Wheldon, M., 2000. Dose-response relationship for radiation-induced mutations at micro- and minisatellite loci in human somatic cells in culture.

International Journal of Radiation Biology. 76, 169-176.

Boyd, M., Livingstone, A., Wilson, L.E., Marshall, E.M., McCluskey, A.G., Mairs, R.J., Wheldon, T.E., 2000. Dose-response relationship for radiation-induced mutations at micro- and minisatellite loci in human somatic cells in culture. International Journal of Radiation Biology. 76, 169-176.

Brain, J.D., Kavet, R., McCormick, D.L., Poole, C., Silverman, L.B., Smith, T.J., Valberg, P.A., Van Etten, R.A., Weaver, J.C., 2003. Childhood leukemia: electric and magnetic fields as possible risk factors. Environmental Health Perspectives. 111, 962-970.

Brocklehurst, B., 2002. Magnetic fields and radical reactions: recent developments and their role in nature. Chemical Society Reviews. 31, 301-311.

Bubenik, G.A., 2002. Gastrointestinal melatonin: localization, function, and clinical relevance. Digestive Diseases and Sciences. 47, 2336-2348.

Bunch, K.J., Keegan, T.J., Swanson, J., Vincent, T.J., Murphy, M.F., 2014. Residential distance at birth from overhead high-voltage powerlines: childhood cancer risk in Britain 1962-2008. British Journal of Cancer. 110, 1402-1408.

Burdak-Rothkamm, S., Rothkamm, K., Folkard, M., Patel, G., Hone, P., Lloyd, D., Ainsbury, L., Prise, K.M., 2009. DNA and chromosomal damage in response to intermittent extremely low-frequency magnetic fields. Mutation Research. 672, 82-89.

Burke, T. \& Bruford, M.W., 1987. DNA fingerprinting in birds. Nature. 327, 149-152.

Carrillo-Vico, A., Calvo, J.R., Abreu, P., Lardone, P.J., Garcia-Maurino, S., Reiter, R.J., Guerrero, J.M., 2004. Evidence of melatonin synthesis by human lymphocytes and its physiological significance: possible role as intracrine, autocrine, and/or paracrine substance. FASEB Journal : Official Publication of the Federation of American Societies for Experimental Biology. 18, 537-539.

Chand, R., Israil, M., Rai, J., 2009. Schumann resonance frequency variations observed in magnetotelluric data recorded from Garhwal Himalayan region India. Annales Geophysicae. 27, 3497-3507.

Chen, C., Ma, X., Zhong, M., Yu, Z., 2010. Extremely low-frequency electromagnetic fields exposure and female breast cancer risk: a meta-analysis based on 24,338 cases and 60,628 controls. Breast Cancer Research and Treatment. 123, 569-576.

Chen, G., Lu, D., Chiang, H., Leszczynski, D., Xu, Z., 2012. Using model organism Saccharomyces cerevisiae to evaluate the effects of ELF-MF and RF-EMF exposure on global gene expression. Bioelectromagnetics. 33, 550-560. 
Cherry, N., 2002. Schumann Resonances, a plausible biophysical mechanism for the human health effects of Solar. Natural Hazards. 26, 279-331.

Chiaretti, S., Gianfelici, V., Ceglie, G., Foa, R., 2014. Genomic characterization of acute leukemias. Medical Principles and Practice : International Journal of the Kuwait University, Health Science Centre. 23, 487-506.

Cho, Y.H. \& Chung, H.W., 2003. The effect of extremely low frequency electromagnetic fields (ELF-EMF) on the frequency of micronuclei and sister chromatid exchange in human lymphocytes induced by benzo(a)pyrene. Toxicology Letters. 143, 37-44.

Chomczynski, P. \& Sacchi, N., 2006. The single-step method of RNA isolation by acid guanidinium thiocyanate-phenol-chloroform extraction: twenty-something years on. Nature Protocols. 1, 581-585.

Chomczynski, P. \& Sacchi, N., 1987. Single-step method of RNA isolation by acid guanidinium thiocyanate-phenol-chloroform extraction. Analytical Biochemistry. 162, 156-159.

Ciejka, E., Kleniewska, P., Skibska, B., Goraca, A., 2011. Effects of extremely low frequency magnetic field on oxidative balance in brain of rats. Journal of Physiology and Pharmacology : An Official Journal of the Polish Physiological Society. 62, 657-661.

Coghill, R.W., Steward, J., Philips, A., 1996. Extra low frequency electric and magnetic fields in the bedplace of children diagnosed with leukaemia: a case-control study. European Journal of Cancer Prevention : The Official Journal of the European Cancer Prevention Organisation (ECP). 5, 153-158.

Coleman, M.P., Bell, C.M., Taylor, H.L., Primic-Zakelj, M., 1989. Leukaemia and residence near electricity transmission equipment: a case-control study. British Journal of Cancer. 60, 793-798.

Coogan, P. \& Aschengrau, A., 1998. Exposure to Power Frequency Magnetic Fields and Risk of Breast Cancer in the Upper Cape Cod Cancer Incidence Study. Archives of Environmental Health: An International Journal. 53, 359-367.

Cooke, H.J. \& Saunders, P.T., 2002. Mouse models of male infertility. Nature Reviews.Genetics. 3, 790-801.

Cooper, A.R., Van Wijngaarden, E., Fisher, S.G., Adams, M.J., Yost, M.G., Bowman, J.D., 2009. A Population-Based Cohort Study of Occupational Exposure to Magnetic Fields and Cardiovascular Disease Mortality. Annals of Epidemiology. 19, 42-48.

Coulton, L.A., Harris, P.A., Barker, A.T., Pockley, A.G., 2004. Effect of $50 \mathrm{~Hz}$ electromagnetic fields on the induction of heat-shock protein gene expression in human leukocytes. Radiation Research. 161, 430-434. 
Davanipour, Z., Sobel, E., Bowman, J.D., Qian, Z., Will, A.D., 1997. Amyotrophic lateral sclerosis and occupational exposure to electromagnetic fields. Bioelectromagnetics. 18, 28-35.

Davis, A.P. \& Justice, M.J., 1998. An Oak Ridge legacy: the specific locus test and its role in mouse mutagenesis. Genetics. 148, 7-12.

de Rooij, D.G. \& Russell, L.D., 2000. All you wanted to know about spermatogonia but were afraid to ask. Journal of Andrology. 21, 776-798.

Ding, G.R., Nakahara, T., Hirose, H., Koyama, S., Takashima, Y., Miyakoshi, J., 2004. Extremely low frequency magnetic fields and the promotion of $\mathrm{H} 2 \mathrm{O} 2$-induced cell death in HL-60 cells. International Journal of Radiation Biology. 80, 317-324.

Dockerty, J.D., Elwood, J.M., Herbison, G.P., 1998. Electromagnetic Field Exposures and Childhood Cancers in New Zealand. Cancer Causes \& Control. 9, 299-309.

Doll, R. \& Wakeford, R., 1997. Risk of childhood cancer from fetal irradiation. The British Journal of Radiology. 70, 130-139.

Draper, G., Vincent, T., Kroll, M.E., Swanson, J., 2005. Childhood cancer in relation to distance from high voltage power lines in England and Wales: a case-control study. BMJ (Clinical Research Ed.). 330, 1290.

Duan, W., Liu, C., Wu, H., Chen, C., Zhang, T., Gao, P., Luo, X., Yu, Z., Zhou, Z., 2014. Effects of exposure to extremely low frequency magnetic fields on spermatogenesis in adult rats. Bioelectromagnetics. 35, 58-69.

Dubrova, Y.E., 2005. Radiation-induced mutation at tandem repeat DNA Loci in the mouse germline: spectra and doubling doses. Radiation Research. 163, 200-207.

Dubrova, Y.E., Grant, G., Chumak, A.A., Stezhka, V.A., Karakasian, A.N., 2002. Elevated minisatellite mutation rate in the post-chernobyl families from ukraine. American Journal of Human Genetics. 71, 801-809.

Dubrova, Y.E., Hickenbotham, P., Glen, C.D., Monger, K., Wong, H.P., Barber, R.C., 2008. Paternal exposure to ethylnitrosourea results in transgenerational genomic instability in mice. Environmental and Molecular Mutagenesis. 49, 308-311.

Dubrova, Y.E., Jeffreys, A.J., Malashenko, A.M., 1993. Mouse minisatellite mutations induced by ionizing radiation. Nature Genetics. 5, 92-94.

Dubrova, Y.E., Nesterov, V.N., Krouchinsky, N.G., Ostapenko, V.A., Neumann, R., Neil, D.L., Jeffreys, A.J., 1996. Human minisatellite mutation rate after the Chernobyl accident. Nature. 380, 683-686.

Dubrova, Y.E., Nesterov, V.N., Krouchinsky, N.G., Ostapenko, V.A., Vergnaud, G., Giraudeau, F., Buard, J., Jeffreys, A.J., 1997. Further evidence for elevated human 
minisatellite mutation rate in Belarus eight years after the Chernobyl accident. Mutation Research. 381, 267-278.

Dubrova, Y.E., Plumb, M., Brown, J., Boulton, E., Goodhead, D., Jeffreys, A.J., 2000a. Induction of minisatellite mutations in the mouse germline by low-dose chronic exposure to gamma-radiation and fission neutrons. Mutation Research. 453, 17-24.

Dubrova, Y.E., Plumb, M., Brown, J., Fennelly, J., Bois, P., Goodhead, D., Jeffreys, A.J., 1998a. Stage specificity, dose response, and doubling dose for mouse minisatellite germ-line mutation induced by acute radiation. Proceedings of the National Academy of Sciences of the United States of America. 95, 6251-6255.

Dubrova, Y.E., Plumb, M., Brown, J., Jeffreys, A.J., 1998b. Radiation-induced germline instability at minisatellite loci. International Journal of Radiation Biology. 74, 689-696.

Dubrova, Y.E., Plumb, M., Gutierrez, B., Boulton, E., Jeffreys, A.J., 2000.

Transgenerational mutation by radiation. Nature. 405, 37.

Dubrova, Y.E. \& Plumb, M.A., 2002. lonising radiation and mutation induction at mouse minisatellite loci. The story of the two generations. Mutation Research. 499, 143-150.

Eden, T., 2010. Aetiology of childhood leukaemia. Cancer Treatment Reviews. 36, 286297.

Edwards, R., 1990. Electric jobs 'carry high cancer risk'. The Guardian (1959-2003), pp.20.

Ellegren, H., 2004. Microsatellites: simple sequences with complex evolution. Nature Reviews.Genetics. 5, 435-445.

Elliott, P., Shaddick, G., Douglass, M., de Hoogh, K., Briggs, D.J., Toledano, M.B., 2013. Adult Cancers Near High-voltage Overhead Power Lines. Epidemiology. 24, 184-190.

(EMFs.info)Electric and magnetic fields and health. 2015. EMFs.info Sources. [Online] Available from: http://www.emfs.info/sources/. [Accessed: 02/03/2015].

Erren, T.C., 2001. A meta-analysis of epidemiologic studies of electric and magnetic fields and breast cancer in women and men. Bioelectromagnetics. Suppl 5, S105-19.

Eveson, R.W., Timmel, C.R., Brocklehurst, B., Hore, P.J., McLauchlan, K.A., 2000. The effects of weak magnetic fields on radical recombination reactions in micelles. International Journal of Radiation Biology. 76, 1509-1522.

Fan, Y.J., Wang, Z., Sadamoto, S., Ninomiya, Y., Kotomura, N., Kamiya, K., Dohi, K., Kominami, R., Niwa, O., 1995. Dose-response of a radiation induction of a germline mutation at a hypervariable mouse minisatellite locus. International Journal of Radiation Biology. 68, 177-183. 
Farag, A.S., Dawoud, M.M., Selim, S.Z., Cheng, T.C., Marcus, A.M., Penn, D., 1998. Electromagnetic fields in the home. Electric Power Systems Research. 45, 73-89.

Fatigoni, C., Dominici, L., Moretti, M., Villarini, M., Monarca, S., 2005. Genotoxic effects of extremely low frequency (ELF) magnetic fields (MF) evaluated by the Tradescantiamicronucleus assay. Environmental Toxicology. 20, 585-591.

Favor, J., 1999. Mechanisms of mutation induction in germ cells of the mouse as assessed by the specific locus test. Mutation Research. 428, 227-236.

Feychting, M. \& Ahlbom, A., 1995. Childhood leukemia and residential exposure to weak extremely low frequency magnetic fields. Environmental Health Perspectives. 103 Suppl 2, 59-62.

Feychting, M. \& Ahlbom, A., 1993. Magnetic fields and cancer in children residing near Swedish high-voltage power lines. American Journal of Epidemiology. 138, 467-481.

Feychting, M., Ahlbom, A., Kheifets, L., 2005. EMF and health. Annual Review of Public Health. 26, 165-189.

Feychting, M., 2013. Invited commentary: extremely low-frequency magnetic fields and breast cancer--now it is enough. American Journal of Epidemiology. 178, 1046.

Feychting, M., Forssén, U., Rutqvist, L.E., Ahlbom, A., 1998. Magnetic Fields and Breast Cancer in Swedish Adults Residing near High-Voltage Power Lines. Epidemiology. 9, 392-397.

Feychting, M., Jonsson, F., Pedersen, N.L., Ahlbom, A., 2003. Occupational Magnetic Field Exposure and Neurodegenerative Disease. Epidemiology. 14, 413-419.

Feychting, M., Pedersen, N.L., Svedberg, P., Floderus, B., Gatz, M., 1998. Dementia and occupational exposure to magnetic fields. Scandinavian Journal of Work, Environment \& Health. 24, 46-53.

Floderus, B., Persson, T., Stenlund, C., Wennberg, A., Ost, A., Knave, B., 1993. Occupational exposure to electromagnetic fields in relation to leukemia and brain tumors: a case-control study in Sweden. Cancer Causes \& Control : CCC. 4, 465-476.

Focke, F., Schuermann, D., Kuster, N., Schar, P., 2010. DNA fragmentation in human fibroblasts under extremely low frequency electromagnetic field exposure. Mutation Research. 683, 74-83.

Fonseca, N.A., Marioni, J., Brazma, A., 2014. RNA-Seq gene profiling-a systematic empirical comparison. PloS One. 9, e107026.

Forssén, U.M., Rutqvist, L.E., Ahlbom, A., Feychting, M., 2005. Occupational magnetic fields and female breast cancer: a case-control study using Swedish population registers and new exposure data. American Journal of Epidemiology. 161, 250-259. 
Frazier, M.E., Reese, J.A., Morris, J.E., Jostes, R.F., Miller, D.L., 1990. Exposure of mammalian cells to $60-\mathrm{Hz}$ magnetic or electric fields: analysis of DNA repair of induced, single-strand breaks. Bioelectromagnetics. 11, 229-234.

Fukuda, H., Katahira, M., Tsuchiya, N., Enokizono, Y., Sugimura, T., Nagao, M., Nakagama, H., 2002. Unfolding of quadruplex structure in the G-rich strand of the minisatellite repeat by the binding protein UP1. Proceedings of the National Academy of Sciences of the United States of America. 99, 12685-12690.

Funk, R.H., Monsees, T., Ozkucur, N., 2009. Electromagnetic effects - From cell biology to medicine. Progress in Histochemistry and Cytochemistry. 43, 177-264.

Galt, S., Wahlström, J., Hamnerius, Y., Holmgvist, D., Johannesson, T., 1995. Study of effects of $50 \mathrm{~Hz}$ magnetic fields on chromosome aberrations and the growth-related enzyme ODC in human amniotic cells. Bioelectrochemistry and Bioenergetics. 36, 1-8.

Gammon, M.D., Schoenberg, J.B., Britton, J.A., Kelsey, J.L., Stanford, J.L., Malone, K.E., Coates, R.J., Brogan, D.J., Potischman, N., Swanson, C.A., Brinton, L.A., 1998. Electric blanket use and breast cancer risk among younger women. American Journal of Epidemiology. 148, 556.

Garcia, J.J., Lopez-Pingarron, L., Almeida-Souza, P., Tres, A., Escudero, P., Garcia-Gil, F.A., Tan, D.X., Reiter, R.J., Ramirez, J.M., Bernal-Perez, M., 2014. Protective effects of melatonin in reducing oxidative stress and in preserving the fluidity of biological membranes: a review. Journal of Pineal Research. 56, 225-237.

Gibbs, M., Collick, A., Kelly, R.G., Jeffreys, A.J., 1993. A tetranucleotide repeat mouse minisatellite displaying substantial somatic instability during early preimplantation development. Genomics. 17, 121-128.

Gilbert, D.A., O'Brien, S.J., Lehman, N., Wayne, R.K., 1990. Genetic fingerprinting reflects population differentiation in the California Channel Island fox. Nature. 344, 764-767.

Glen, C.D. \& Dubrova, Y.E., 2012. Exposure to anticancer drugs can result in transgenerational genomic instability in mice. Proceedings of the National Academy of Sciences of the United States of America. 109, 2984-2988.

Glen, C.D., McVeigh, L.E., Voutounou, M., Dubrova, Y.E., 2015. The effects of methyldonor deficiency on the pattern of gene expression in mice. Molecular Nutrition \& Food Research. 59, 501-506.

Glen, C.D., Smith, A.G., Dubrova, Y.E., 2008. Single-molecule PCR analysis of germ line mutation induction by anticancer drugs in mice. Cancer Research. 68, 3630-3636.

Gomes, A.M., Barber, R.C., Dubrova, Y.E., 2015. Paternal irradiation perturbs the expression of circadian genes in offspring. Mutation Research. 775, 33-37. 
Graham, C., Cook, M.R., Sastre, A., Gerkovich, M.M., Kavet, R., 2000. Cardiac autonomic control mechanisms in power-frequency magnetic fields: a multistudy analysis. Environmental Health Perspectives. 108, 737-742.

Greaves, M., 2006. Infection, immune responses and the aetiology of childhood leukaemia. Nature Reviews.Cancer. 6, 193-203.

Greaves, M., 2002. Childhood leukaemia. BMJ (Clinical Research Ed.). 324, 283-287.

Greaves, M., 1999. Molecular genetics, natural history and the demise of childhood leukaemia. European Journal of Cancer (Oxford, England : 1990). 35, 1941-1953.

Greaves, M.F. \& Wiemels, J., 2003a. Origins of chromosome translocations in childhood leukaemia. Nature Reviews.Cancer. 3, 639-649.

Green, S., Lavappa, K.S., Manandhar, M., Sheu, C.J., Whorton, E., Springer, J.A., 1987. A guide for mutagenicity testing using the dominant lethal assay. Mutation Research. 189, 167-174.

Greenland, S., Sheppard, A.R., Kaune, W.T., Poole, C., Kelsh, M.A., 2000. A pooled analysis of magnetic fields, wire codes, and childhood leukemia. Epidemiology. 11, 624634.

Grellier, J., Ravazzani, P., Cardis, E., 2014. Potential health impacts of residential exposures to extremely low frequency magnetic fields in Europe. Environment International. 62, 55-63.

Gross, C.G., 2000. Neurogenesis in the adult brain: death of a dogma. Nature Reviews.Neuroscience. 1, 67-73.

Gurney, J.G., Mueller, B.A., Davis, S., Schwartz, S.M., Stevens, R.G., Kopecky, K.J., 1996. Childhood brain tumor occurrence in relation to residential power line configurations, electric heating sources, and electric appliance use. American Journal of Epidemiology. 143, 120.

Gurney, J.G., Severson, R.K., Davis, S., Robison, L.L., 1995. Incidence of cancer in children in the United States. Sex-, race-, and 1-year age-specific rates by histologic type. Cancer. 75, 2186-2195.

Hakansson, N., Gustavsson, P., Johansen, C., Floderus, B., 2003. Neurodegenerative diseases in welders and other workers exposed to high levels of magnetic fields. Epidemiology. 14, 420-6.

Håkansson, N., Gustavsson, P., Sastre, A., Floderus, B., 2003. Occupational exposure to extremely low frequency magnetic fields and mortality from cardiovascular disease. American Journal of Epidemiology. 158, 534-542. 
Hanneman, W.H., Legare, M.E., Sweeney, S., Schimenti, J.C., 1997. Cisplatin increases meiotic crossing-over in mice. Proceedings of the National Academy of Sciences of the United States of America. 94, 8681-8685.

Hardeland, R., 2005. Antioxidative protection by melatonin: multiplicity of mechanisms from radical detoxification to radical avoidance. Endocrine. 27, 119-130.

Hardwick, R.J., Tretyakov, M.V., Dubrova, Y.E., 2009. Age-related accumulation of mutations supports a replication-dependent mechanism of spontaneous mutation at tandem repeat DNA Loci in mice. Molecular Biology and Evolution. 26, 2647-2654.

Hatch, E.E., Linet, M.S., Kleinerman, R.A., Tarone, R.E., Severson, R.K., Hartsock, C.T., Haines, C., Kaune, W.T., Friedman, D., Robison, L.L., Wacholder, S., 1998. Association between childhood acute lymphoblastic leukemia and use of electrical appliances during pregnancy and childhood. Epidemiology. 9, 234-245.

Hatch, T., Derijck, A.A., Black, P.D., van der Heijden, G.W., de Boer, P., Dubrova, Y.E., 2007. Maternal effects of the scid mutation on radiation-induced transgenerational instability in mice. Oncogene. 26, 4720-4724.

He, Y., Jiang, X., Chen, J., 2014. The role of miR-150 in normal and malignant hematopoiesis. Oncogene. 33, 3887-3893.

Henderson, B., Kind, M., Boeck, G., Helmberg, A., Wick, G., 2006. Gene expression profiling of human endothelial cells exposed to $50-\mathrm{Hz}$ magnetic fields fails to produce regulated candidate genes. Cell Stress \& Chaperones. 11, 227-232.

Henshaw, D.L. \& Reiter, R.J., 2005. Do magnetic fields cause increased risk of childhood leukemia via melatonin disruption? Bioelectromagnetics. Suppl 7, S86-97.

Hill, S.M., Blask, D.E., Xiang, S., Yuan, L., Mao, L., Dauchy, R.T., Dauchy, E.M., Frasch, T., Duplesis, T., 2011. Melatonin and associated signaling pathways that control normal breast epithelium and breast cancer. Journal of Mammary Gland Biology and Neoplasia. 16, 235-245.

Hong, M.N., Han, N.K., Lee, H.C., Ko, Y.K., Chi, S.G., Lee, Y.S., Gimm, Y.M., Myung, S.H., Lee, J.S., 2012. Extremely low frequency magnetic fields do not elicit oxidative stress in MCF10A cells. Journal of Radiation Research. 53, 79-86.

Hore, P.J., 2012. Are biochemical reactions affected by weak magnetic fields? Proceedings of the National Academy of Sciences of the United States of America. 109, 1357-1358.

Huuskonen, H., Lindbohm, M.L., Juutilainen, J., 1998. Teratogenic and reproductive effects of low-frequency magnetic fields. Mutation Research. 410, 167-183.

IARC Working Group on the Evaluation of Carcinogenic Risks to Humans, 2000. Ionizing radiation, Part I, X-and gamma-radiation and neutrons. IARC Monographs on the 
Evaluation of Carcinogenic Risks to Humans / World Health Organization, International Agency for Research on Cancer. 75 Pt 1, 1-448.

IARC Working Group on the Evaluation of Carcinogenic Risks to Humans, 2002. Nonionizing radiation, Part 1: static and extremely low-frequency (ELF) electric and magnetic fields. IARC Monographs on the Evaluation of Carcinogenic Risks to Humans / World Health Organization, International Agency for Research on Cancer. 80, 1-395.

IARC Working Group on the Evaluation of Carcinogenic Risks to Humans, 2013. Nonionizing radiation, Part 2: Radiofrequency electromagnetic fields. IARC Monographs on the Evaluation of Carcinogenic Risks to Humans / World Health Organization, International Agency for Research on Cancer. 102, 1-460.

IARC Working Group on the Evaluation of Carcinogenic Risks to Humans, 2016. IARC Monographs on the Evaluation of Carcinogenic Risks to Humans / World Health Organization, International Agency for Research on Cancer - List of Classifications, Volumes 1-115. [Online]. [Accessed 23 March 2016]. Available from: http://monographs.iarc.fr/ENG/Classification/latest_classif.php

Inaba, H., Greaves, M., Mullighan, C.G., 2013. Acute lymphoblastic leukaemia. Lancet. 381, 1943-1955.

International Commission on Non-lonizing Radiation Protection, 2010. Guidelines for limiting exposure to time-varying electric and magnetic fields ( $1 \mathrm{~Hz}$ to $100 \mathrm{kHz}$ ). Health Physics. 99, 818-836.

International Commission on Non-lonizing Radiation Protection, 1998. "Guidelines for limiting exposure to time-varying electric, magnetic, and electromagnetic fields (up to $300 \mathrm{GHz}) "$. Health Physics. 74, 494-522.

Ivancsits, S., Diem, E., Jahn, O., Rudiger, H.W., 2003a. Age-related effects on induction of DNA strand breaks by intermittent exposure to electromagnetic fields. Mechanisms of Ageing and Development. 124, 847-850.

Ivancsits, S., Diem, E., Jahn, O., Rudiger, H.W., 2003b. Intermittent extremely low frequency electromagnetic fields cause DNA damage in a dose-dependent way. International Archives of Occupational and Environmental Health. 76, 431-436.

Ivancsits, S., Diem, E., Pilger, A., Rudiger, H.W., Jahn, O., 2002. Induction of DNA strand breaks by intermittent exposure to extremely-low-frequency electromagnetic fields in human diploid fibroblasts. Mutation Research. 519, 1-13.

Ivancsits, S., Pilger, A., Diem, E., Jahn, O., Rudiger, H.W., 2005. Cell type-specific genotoxic effects of intermittent extremely low-frequency electromagnetic fields. Mutation Research. 583, 184-188. 
Jackson, J.D., 1992. Are the stray $60-\mathrm{Hz}$ electromagnetic fields associated with the distribution and use of electric power a significant cause of cancer? Proceedings of the National Academy of Sciences of the United States of America. 89, 3508-3510.

Jeffreys, A.J., 1987. Highly variable minisatellites and DNA fingerprints. Biochemical Society Transactions. 15, 309-317.

Jeffreys, A.J., Barber, R., Bois, P., Buard, J., Dubrova, Y.E., Grant, G., Hollies, C.R., May, C.A., Neumann, R., Panayi, M., Ritchie, A.E., Shone, A.C., Signer, E., Stead, J.D., Tamaki, K., 1999. Human minisatellites, repeat DNA instability and meiotic recombination.

Electrophoresis. 20, 1665-1675.

Jeffreys, A.J., Royle, N.J., Wilson, V., Wong, Z., 1988. Spontaneous mutation rates to new length alleles at tandem-repetitive hypervariable loci in human DNA. Nature. 332, 278-281.

Jeffreys, A.J., Tamaki, K., MacLeod, A., Monckton, D.G., Neil, D.L., Armour, J.A., 1994. Complex gene conversion events in germline mutation at human minisatellites. Nature Genetics. 6, 136-145.

Jeffreys, A.J., Turner, M., Debenham, P., 1991. The efficiency of multilocus DNA fingerprint probes for individualization and establishment of family relationships, determined from extensive casework. American Journal of Human Genetics. 48, 824840 .

Jeffreys, A.J., Wilson, V., Kelly, R., Taylor, B.A., Bulfield, G., 1987. Mouse DNA 'fingerprints': analysis of chromosome localization and germ-line stability of hypervariable loci in recombinant inbred strains. Nucleic Acids Research. 15, 28232836.

Jeffreys, A.J., Wilson, V., Thein, S.L., 1985a. Hypervariable 'minisatellite' regions in human DNA. Nature. 314, 67-73.

Jeffreys, A.J., Wilson, V., Thein, S.L., 1985b. Individual-specific 'fingerprints' of human DNA. Nature. 316, 76-79.

Jin, Y.B., Choi, S.H., Lee, J.S., Kim, J.K., Lee, J.W., Hong, S.C., Myung, S.H., Lee, Y.S., 2014. Absence of DNA damage after $60-\mathrm{Hz}$ electromagnetic field exposure combined with ionizing radiation, hydrogen peroxide, or c-Myc overexpression. Radiation and Environmental Biophysics. 53, 93-101.

Jin, Y.B., Kang, G.Y., Lee, J.S., Choi, J.I., Lee, J.W., Hong, S.C., Myung, S.H., Lee, Y.S., 2012. Effects on micronuclei formation of $60-\mathrm{Hz}$ electromagnetic field exposure with ionizing radiation, hydrogen peroxide, or c-Myc overexpression. International Journal of Radiation Biology. 88, 374-380.

Johansen, C., 2000. Exposure to electromagnetic fields and risk of central nervous system disease in utility workers. Epidemiology. 11, 539-543. 
Johansen, C., Feychting, M., Møller, M., Arnsbo, P., Ahlbom, A., Olsen, J.H., 2002. Risk of severe cardiac arrhythmia in male utility workers: a nationwide Danish cohort study. American Journal of Epidemiology. 156, 857-861.

Juutilainen, J., 2003. Developmental effects of extremely low frequency electric and magnetic fields. Radiation Protection Dosimetry. 106, 385-390.

Juutilainen, J., Kumlin, T., Naarala, J., 2006. Do extremely low frequency magnetic fields enhance the effects of environmental carcinogens? A meta-analysis of experimental studies. International Journal of Radiation Biology. 82, 1-12.

Juutilainen, J., Lang, S., Rytomaa, T., 2000. Possible cocarcinogenic effects of ELF electromagnetic fields may require repeated long-term interaction with known carcinogenic factors. Bioelectromagnetics. 21, 122-128.

Juutilainen, J., 2005. Developmental effects of electromagnetic fields. Bioelectromagnetics. 26, S107-S115.

Kabat, G.C., O'Leary, E.S., Schoenfeld, E.R., Greene, J.M., Grimson, R., Henderson, K., Kaune, W.T., Gammon, M.D., Britton, J.A., Teitelbaum, S.L., Neugut, A.I., Leske, M.C., EBCLIS Group, 2003. Electric Blanket Use and Breast Cancer on Long Island. Epidemiology. 14, 514-520.

Kang, S., Ohshima, K., Shimizu, M., Amirhaeri, S., Wells, R.D., 1995. Pausing of DNA synthesis in vitro at specific loci in CTG and CGG triplet repeats from human hereditary disease genes. The Journal of Biological Chemistry. 270, 27014-27021.

Kannan, K. \& Jain, S.K., 2000. Oxidative stress and apoptosis. Pathophysiology: The Official Journal of the International Society for Pathophysiology / ISP. 7, 153-163.

Karipidis, K.K., 2015. Survey of residential power-frequency magnetic fields in Melbourne, Australia. Radiation Protection Dosimetry. 163, 81-91.

Kato, M., Honma, K., Shigemitsu, T., Shiga, Y., 1994a. Circularly polarized 50-Hz magnetic field exposure reduces pineal gland and blood melatonin concentrations of Long-Evans rats. Neuroscience Letters. 166, 59-62.

Kato, M., Honma, K., Shigemitsu, T., Shiga, Y., 1994b. Circularly polarized, sinusoidal, $50 \mathrm{~Hz}$ magnetic field exposure does not influence plasma testosterone levels of rats. Bioelectromagnetics. 15, 513-518.

Kato, M., Honma, K., Shigemitsu, T., Shiga, Y., 1994c. Horizontal or vertical 50-Hz, 1microT magnetic fields have no effect on pineal gland or plasma melatonin concentration of albino rats. Neuroscience Letters. 168, 205-208.

Kato, M., Honma, K., Shigemitsu, T., Shiga, Y., 1994d. Recovery of nocturnal melatonin concentration takes place within one week following cessation of $50 \mathrm{~Hz}$ circularly polarized magnetic field exposure for six weeks. Bioelectromagnetics. 15, 489-492. 
Kato, M., Honma, K., Shigemitsu, T., Shiga, Y., 1993. Effects of exposure to a circularly polarized $50-\mathrm{Hz}$ magnetic field on plasma and pineal melatonin levels in rats.

Bioelectromagnetics. 14, 97-106.

Katoh, M.A., Cain, K.T., Hughes, L.A., Foxworth, L.B., Bishop, J.B., Generoso, W.M., 1990. Female-specific dominant lethal effects in mice. Mutation Research. 230, 205217.

Kaune, W.T., 1993. Assessing human exposure to power-frequency electric and magnetic fields. Environmental Health Perspectives. 101 Suppl 4, 121-133.

Kaune, W.T. \& Zaffanella, L.E., 1994. Assessing historical exposures of children to power-frequency magnetic fields. Journal of Exposure Analysis and Environmental Epidemiology. 4, 149-170.

Kelly, R., Bulfield, G., Collick, A., Gibbs, M., Jeffreys, A.J., 1989. Characterization of a highly unstable mouse minisatellite locus: evidence for somatic mutation du ring early development. Genomics. 5, 844-856.

Kelly, R., Gibbs, M., Collick, A., Jeffreys, A.J., 1991. Spontaneous mutation at the hypervariable mouse minisatellite locus Ms6-hm: flanking DNA sequence and analysis of germline and early somatic mutation events. Proceedings. Biological Sciences / the Royal Society. 245, 235-245.

Kelly, R.G., 1994. Similar origins of two mouse minisatellites within transposon-like LTRs. Genomics. 24, 509-515.

Kheifets, L.I., 2001. Electric and magnetic field exposure and brain cancer: a review. Bioelectromagnetics. Suppl 5, S120.

Kheifets, L.I., Kavet, R., Sussman, S.S., 1997. Wire codes, magnetic fields, and childhood cancer. Bioelectromagnetics. 18, 99-110.

Kheifets, L., Ahlbom, A., Crespi, C.M., Draper, G., Hagihara, J., Lowenthal, R.M., Mezei, G., Oksuzyan, S., Schuz, J., Swanson, J., Tittarelli, A., Vinceti, M., Wunsch Filho, V., 2010. Pooled analysis of recent studies on magnetic fields and childhood leukaemia. British Journal of Cancer. 103, 1128-1135.

Kheifets, L., Mezei, G., Greenland, S., 2006. Comment concerning "Childhood leukemia and residential magnetic fields: are pooled analyses more valid than the original studies?" (Bioelectromagnetics 27:1-7 [2006]). Bioelectromagnetics. 27, 674-5; discussion 675-6.

Kheifets, L. \& Oksuzyan, S., 2008. Exposure assessment and other challenges in nonionizing radiation studies of childhood leukaemia. Radiation Protection Dosimetry. 132, 139-147. 
Kheifets, L., Repacholi, M., Saunders, R., van Deventer, E., 2005. The sensitivity of children to electromagnetic fields. Pediatrics. 116, 303-13.

Kheifets, L.I., Gilbert, E.S., Sussman, S.S., Guenel, P., Sahl, J.D., Savitz, D.A., Theriault, G., 1999. Comparative analyses of the studies of magnetic fields and cancer in electric utility workers: studies from France, Canada, and the United States. Occupational and Environmental Medicine. 56, 567-574.

Kheifets, L., Ahlbom, A., Johansen, C., Feychting, M., Sahl, J., Savitz, D., 2007. Extremely low-frequency magnetic fields and heart disease. Scandinavian Journal of Work, Environment \& Health. 33, 5-12.

Kim, H.S., Park, B.J., Jang, H.J., Ipper, N.S., Kim, S.H., Kim, Y.J., Jeon, S.H., Lee, K.S., Lee, S.K., Kim, N., Ju, Y.J., Gimm, Y.M., Kim, Y.W., 2014. Continuous exposure to $60 \mathrm{~Hz}$ magnetic fields induces duration- and dose-dependent apoptosis of testicular germ cells. Bioelectromagnetics. 35, 100-107.

Kim, J., Ha, C.S., Lee, H.J., Song, K., 2010. Repetitive exposure to a 60-Hz time-varying magnetic field induces DNA double-strand breaks and apoptosis in human cells. Biochemical and Biophysical Research Communications. 400, 739-744.

Kim, Y.W., Kim, H.S., Lee, J.S., Kim, Y.J., Lee, S.K., Seo, J.N., Jung, K.C., Kim, N., Gimm, Y.M., 2009. Effects of $60 \mathrm{~Hz} 14$ microT magnetic field on the apoptosis of testicular germ cell in mice. Bioelectromagnetics. 30, 66-72.

Kirk, K.M. \& Lyon, M.F., 1984. Induction of congenital malformations in the offspring of male mice treated with $\mathrm{X}$-rays at pre-meiotic and post-meiotic stages. Mutation Research. 125, 75-85.

Kiuru, A., Auvinen, A., Luokkamaki, M., Makkonen, K., Veidebaum, T., Tekkel, M., Rahu, M., Hakulinen, T., Servomaa, K., Rytomaa, T., Mustonen, R., 2003. Hereditary minisatellite mutations among the offspring of Estonian Chernobyl cleanup workers. Radiation Research. 159, 651-655.

Kleinerman, R.A., Kaune, W.T., Hatch, E.E., Wacholder, S., Linet, M.S., Robison, L.L., Niwa, S., Tarone, R.E., 2000. Are children living near high-voltage power lines at increased risk of acute lymphoblastic leukemia? American Journal of Epidemiology. 151, 512-515.

Kleinerman, R.A., Linet, M.S., Hatch, E.E., Tarone, R.E., Black, P.M., Selker, R.G., Shapiro, W.R., Fine, H.A., Inskip, P.D., 2005. Self-reported electrical appliance use and risk of adult brain tumors. American Journal of Epidemiology. 161, 136-146.

Kliukiene, J., Tynes, T., Andersen, A., 2004. Residential and occupational exposures to $50-\mathrm{Hz}$ magnetic fields and breast cancer in women: a population-based study. American Journal of Epidemiology. 159, 852-861. 
Kodaira, M., Izumi, S., Takahashi, N., Nakamura, N., 2004. No evidence of radiation effect on mutation rates at hypervariable minisatellite loci in the germ cells of atomic bomb survivors. Radiation Research. 162, 350-356.

Kodaira, M., Satoh, C., Hiyama, K., Toyama, K., 1995. Lack of effects of atomic bomb radiation on genetic instability of tandem-repetitive elements in human germ cells. American Journal of Human Genetics. 57, 1275-1283.

Koeman, T., Slottje, P., Kromhout, H., Schouten, L.J., Goldbohm, R.A., van den Brandt, P.A., Vermeulen, R., 2013. Occupational exposure to extremely low-frequency magnetic fields and cardiovascular disease mortality in a prospective cohort study. Occupational and Environmental Medicine. 70, 402.

Kroll, M.E., Swanson, J., Vincent, T.J., Draper, G.J., 2010a. Childhood cancer and magnetic fields from high-voltage power lines in England and Wales: a case-control study. British Journal of Cancer. 103, 1122-1127.

Kumlin, T., Kosma, V.M., Alhonen, L., Janne, J., Komulainen, H., Lang, S., Rytomaa, T., Servomaa, K., Juutilainen, J., 1998. Effects of $50 \mathrm{~Hz}$ magnetic fields on UV-induced skin tumourigenesis in ODC-transgenic and non-transgenic mice. International Journal of Radiation Biology. 73, 113-121.

Kuo, L.J. \& Yang, L.X., 2008. Gamma-H2AX - a novel biomarker for DNA double-strand breaks. In Vivo. 22, 305-309.

Lacy-Hulbert, A., Metcalfe, J.C., Hesketh, R., 1998. Biological responses to electromagnetic fields. FASEB Journal : Official Publication of the Federation of American Societies for Experimental Biology. 12, 395-420.

Laden, F., Neas, L.M., Tolbert, P.E., Holmes, M.D., Hankinson, S.E., Spiegelman, D., Speizer, F.E., Hunter, D.J., 2000. Electric blanket use and breast cancer in the Nurses' Health Study. American Journal of Epidemiology. 152, 41-49.

Lagiou, P., Tamimi, R., Lagiou, A., Mucci, L., Trichopoulos, D., 2002. Is epidemiology implicating extremely low frequency electric and magnetic fields in childhood leukemia? Environmental Health and Preventive Medicine. 7, 33-39.

Lai, H. \& Singh, N.P., 1997. Melatonin and N-tert-butyl-alpha-phenylnitrone block 60$\mathrm{Hz}$ magnetic field-induced DNA single and double strand breaks in rat brain cells. Journal of Pineal Research. 22, 152.

Lai, H. \& Singh, N.P., 2004. Magnetic-field-induced DNA strand breaks in brain cells of the rat. Environmental Health Perspectives. 112, 687-694.

Lai, H. \& Singh, N.P., 1997. Acute exposure to a $60 \mathrm{~Hz}$ magnetic field increases DNA strand breaks in rat brain cells. Bioelectromagnetics. 18, 156-165. 
Lai, H. \& Singh, N.P., 1996. Single- and double-strand DNA breaks in rat brain cells after acute exposure to radiofrequency electromagnetic radiation. International Journal of Radiation Biology. 69, 513-521.

Lara, P.C., Lopez-Penalver, J.J., Farias Vde, A., Ruiz-Ruiz, M.C., Oliver, F.J., Ruiz de Almodovar, J.M., 2015. Direct and bystander radiation effects: a biophysical model and clinical perspectives. Cancer Letters. 356, 5-16.

Lee, G.M., Neutra, R.R., Hristova, L., Yost, M., Hiatt, R.A., 2000. The use of electric bed heaters and the risk of clinically recognized spontaneous abortion. Epidemiology. 11, 406-415.

Lerner, A.B., Case, J.D., Mori, W., Wright, M.R., 1959. Melatonin in peripheral nerve. Nature. 183, 1821.

Lewczuk, B., Redlarski, G., Zak, A., Ziolkowska, N., Przybylska-Gornowicz, B., Krawczuk, M., 2014. Influence of electric, magnetic, and electromagnetic fields on the circadian system: current stage of knowledge. BioMed Research International. 2014, 169459.

Lewy, A.J., Wehr, T.A., Goodwin, F.K., Newsome, D.A., Markey, S.P., 1980. Light suppresses melatonin secretion in humans. Science. 210, 1267-1269.

Li, C.Y., Theriault, G., Lin, R.S., 1997. Residential exposure to 60-Hertz magnetic fields and adult cancers in Taiwan. Epidemiology. 8, 25-30.

Li, J., Hou, R., Niu, X., Liu, R., Wang, Q., Wang, C., Li, X., Hao, Z., Yin, G., Zhang, K., 2016. Comparison of microarray and RNA-Seq analysis of mRNA expression in dermal mesenchymal stem cells. Biotechnology Letters. 38, 33-41.

Li, M., Jones, L., Gaillard, C., Binnewies, M., Ochoa, R., Garcia, E., Lam, V., Wei, G., Yang, W., Lobe, C., Hermiston, M., Passegue, E., Kogan, S.C., 2013. Initially disadvantaged, TEL-AML1 cells expand and initiate leukemia in response to irradiation and cooperating mutations. Leukemia. 27, 1570-1573.

Limon-Pacheco, J. \& Gonsebatt, M.E., 2009. The role of antioxidants and antioxidantrelated enzymes in protective responses to environmentally induced oxidative stress. Mutation Research. 674, 137-147.

Lin, R.S., Dischinger, P.C., Conde, J., Farrell, K.P., 1985. Occupational exposure to electromagnetic fields and the occurrence of brain tumors. An analysis of possible associations. Journal of Occupational Medicine.: Official Publication of the Industrial Medical Association. 27, 413.

Linet, M.S. \& Devesa, S.S., 1991. Descriptive epidemiology of childhood leukaemia. British Journal of Cancer. 63, 424-429. 
Linet, M.S., Ries, L.A., Smith, M.A., Tarone, R.E., Devesa, S.S., 1999. Cancer surveillance series: recent trends in childhood cancer incidence and mortality in the United States. Journal of the National Cancer Institute. 91, 1051-1058.

Linet, M.S., Hatch, E.E., Kleinerman, R.A., Robison, L.L., Kaune, W.T., Friedman, D.R., Severson, R.K., Haines, C.M., Hartsock, C.T., Niwa, S., Wacholder, S., Tarone, R.E., 1997. Residential Exposure to Magnetic Fields and Acute Lymphoblastic Leukemia in Children. The New England Journal of Medicine. 337, 1-8.

Livshits, L.A., Malyarchuk, S.G., Kravchenko, S.A., Matsuka, G.H., Lukyanova, E.M., Antipkin, Y.G., Arabskaya, L.P., Petit, E., Giraudeau, F., Gourmelon, P., Vergnaud, G., Le Guen, B., 2001. Children of chernobyl cleanup workers do not show elevated rates of mutations in minisatellite alleles. Radiation Research. 155, 74-80.

Loberg, L.I., Engdahl, W.R., Gauger, J.R., McCormick, D.L., 2000. Expression of cancerrelated genes in human cells exposed to $60 \mathrm{~Hz}$ magnetic fields. Radiation Research. 153, 679-684.

Loeb, L.A., Loeb, K.R., Anderson, J.P., 2003. Multiple mutations and cancer. Proceedings of the National Academy of Sciences of the United States of America. 100, 776-781.

London, S.J., Thomas, D.C., Bowman, J.D., Sobel, E., Cheng, T.C., Peters, J.M., 1991. Exposure to residential electric and magnetic fields and risk of childhood leukemia. American Journal of Epidemiology. 134, 923-937.

Luceri, C., De Filippo, C., Giovannelli, L., Blangiardo, M., Cavalieri, D., Aglietti, F., Pampaloni, M., Andreuccetti, D., Pieri, L., Bambi, F., Biggeri, A., Dolara, P., 2005. Extremely low-frequency electromagnetic fields do not affect DNA damage and gene expression profiles of yeast and human lymphocytes. Radiation Research. 164, 277 285.

Luning, K.G. \& Searle, A.G., 1971. Estimates of the genetic risks from ionizing irradiation. Mutation Research. 12, 291-304.

Lupke, M., Rollwitz, J., Simkó, M., 2004. Cell Activating Capacity of 50 Hz Magnetic Fields to Release Reactive Oxygen Intermediates in Human Umbilical Cord Bloodderived Monocytes and in Mono Mac 6 Cells. Free Radical Research. 38, 985-993.

Luukkonen, J., Liimatainen, A., Hoyto, A., Juutilainen, J., Naarala, J., 2011. Pre-exposure to $50 \mathrm{~Hz}$ magnetic fields modifies menadione-induced genotoxic effects in human $\mathrm{SH}$ SY5Y neuroblastoma cells. PloS One. 6, 18021.

Luukkonen, J., Liimatainen, A., Juutilainen, J., Naarala, J., 2014. Induction of genomic instability, oxidative processes, and mitochondrial activity by $50 \mathrm{~Hz}$ magnetic fields in human SH-SY5Y neuroblastoma cells. Mutation Research. 760, 33-41. 
Lyon, M.F. \& Morris, T., 1966. Mutation rates at a new set of specific loci in the mouse. Genetical Research. 7, 12-17.

Maes, A., Collier, M., Vandoninck, S., Scarpa, P., Verschaeve, L., 2000. Cytogenetic effects of $50 \mathrm{~Hz}$ magnetic fields of different magnetic flux densities.

Bioelectromagnetics. 21, 589-596.

Mairs, R.J., Hughes, K., Fitzsimmons, S., Prise, K.M., Livingstone, A., Wilson, L., Baig, N., Clark, A.M., Timpson, A., Patel, G., Folkard, M., Angerson, W.J., Boyd, M., 2007. Microsatellite analysis for determination of the mutagenicity of extremely lowfrequency electromagnetic fields and ionising radiation in vitro. Mutation Research. $626,34-41$.

Mandeville, R., Franco, E., Sidrac-Ghali, S., Paris-Nadon, L., Rocheleau, N., Mercier, G., Desy, M., Gaboury, L., 1997. Evaluation of the potential carcinogenicity of $60 \mathrm{~Hz}$ linear sinusoidal continuous-wave magnetic fields in Fischer F344 rats. FASEB Journal : Official Publication of the Federation of American Societies for Experimental Biology. 11, 1127-1136.

Mannerling, A.C., Simko, M., Mild, K.H., Mattsson, M.O., 2010. Effects of 50-Hz magnetic field exposure on superoxide radical anion formation and HSP70 induction in human K562 cells. Radiation and Environmental Biophysics. 49, 731-741.

Marchetti, F., Rowan-Carroll, A., Williams, A., Polyzos, A., Berndt-Weis, M.L., Yauk, C.L., 2011. Sidestream tobacco smoke is a male germ cell mutagen. Proceedings of the National Academy of Sciences of the United States of America. 108, 12811-12814.

McBride, M.L., Gallagher, R.P., Thériault, G., Armstrong, B.G., Tamaro, S., Spinelli, J.J., Deadman, J.E., Fincham, S., Robson, D., Choi, W., 1999. Power-frequency electric and magnetic fields and risk of childhood leukemia in Canada. American Journal of Epidemiology. 149, 831.

McCann, J., Dietrich, F., Rafferty, C., 1998. The genotoxic potential of electric and magnetic fields: an update. Mutation Research. 411, 45-86.

McCormick, D.L., Boorman, G.A., Findlay, J.C., Hailey, J.R., Johnson, T.R., Gauger, J.R., Pletcher, J.M., Sills, R.C., Haseman, J.K., 1999. Chronic toxicity/oncogenicity evaluation of $60 \mathrm{~Hz}$ (power frequency) magnetic fields in B6C3F1 mice. Toxicologic Pathology. 27, 279-285.

McCormick, D.L., Ryan, B.M., Findlay, J.C., Gauger, J.R., Johnson, T.R., Morrissey, R.L., Boorman, G.A., 1998. Exposure to $60 \mathrm{~Hz}$ magnetic fields and risk of lymphoma in PIM transgenic and TSG-p53 (p53 knockout) mice. Carcinogenesis. 19, 1649-1653.

McElroy, J., 2007. Occupational exposure to electromagnetic field and breast cancer risk in a large, population-based, case-control study in the United States. J Occup Environ Med. 49, 266-274. 
McElroy, J.A., Newcomb, P.A., Remington, P.L., Egan, K.M., Titus-Ernstoff, L., TrenthamDietz, A., Hampton, J.M., Baron, J.A., Stampfer, M.J., Willett, W.C., 2001. Electric Blanket or Mattress Cover Use and Breast Cancer Incidence in Women 50-79 Years of Age. Epidemiology. 12, 613-617.

McNamee, J.P., Bellier, P.V., Chauhan, V., Gajda, G.B., Lemay, E., Thansandote, A., 2005. Evaluating DNA damage in rodent brain after acute $60 \mathrm{~Hz}$ magnetic-field exposure. Radiation Research. 164, 791-797.

McNamee, J.P., Bellier, P.V., McLean, J.R., Marro, L., Gajda, G.B., Thansandote, A., 2002. DNA damage and apoptosis in the immature mouse cerebellum after acute exposure to a $1 \mathrm{mT}, 60 \mathrm{~Hz}$ magnetic field. Mutation Research. 513, 121-133.

McNamee, J.P., McLean, J.R., Ferrarotto, C.L., Bellier, P.V., 2000. Comet assay: rapid processing of multiple samples. Mutation Research. 466, 63-69.

Merchant, C.J., Renew, D.C., Swanson, J., 1994. Exposures to power-frequency magnetic fields in the home. Journal of Radiological Protection. 14, 77.

Messiha, H.L., Wongnate, T., Chaiyen, P., Jones, A.R., Scrutton, N.S., 2015. Magnetic field effects as a result of the radical pair mechanism are unlikely in redox enzymes. Journal of the Royal Society, Interface / the Royal Society. 12,: 2014.1155.

Metcalf, D., 1988. The molecular control of blood cells. Harvard University Press.

Mezei, G., Cher, D., Kelsh, M., Edinboro, C., Chapman, P., Kavet, R., 2005. Occupational Magnetic Field Exposure, Cardiovascular Disease Mortality, and Potential Confounding by Smoking. Annals of Epidemiology. 15, 622-629.

Mezei, G., Gadallah, M., Kheifets, L., 2008. Residential Magnetic Field Exposure and Childhood Brain Cancer: A Meta-Analysis. Epidemiology. 19, 424-430.

Mezei, G. \& Kheifets, L., 2006. Selection bias and its implications for case-control studies: a case study of magnetic field exposure and childhood leukaemia. International Journal of Epidemiology. 35, 397-406.

Mezei, G., Sudan, M., Izraeli, S., Kheifets, L., 2014. Epidemiology of childhood leukemia in the presence and absence of Down syndrome. Cancer Epidemiology. 38, 479-489.

Michaelis, J., Schüz, J., Meinert, R., Zemann, E., Grigat, J., Kaatsch, P., Kaletsch, U., Miesner, A., Brinkmann, K., Kalkner, W., Kärner, H., 1998. Combined Risk Estimates for Two German Population-Based Case-Control Studies on Residential Magnetic Fields and Childhood Acute Leukemia. Epidemiology. 9, 92-94.

Milham, S., 1985. Mortality in Workers Exposed to Electromagnetic Fields. Environmental Health Perspectives. 62, 297-300. 
Miller, A.B. \& Green, L.M., 2010. Electric and magnetic fields at power frequencies. Chronic Diseases in Canada. 29 Suppl 1, 69-83.

Mirick, D.K. \& Davis, S., 2008. Melatonin as a biomarker of circadian dysregulation. Cancer Epidemiology, Biomarkers \& Prevention : A Publication of the American Association for Cancer Research, Cosponsored by the American Society of Preventive Oncology. 17, 3306-3313.

Mirkin, S.M., 2007. Expandable DNA repeats and human disease. Nature. 447, 932940.

Miyakoshi, J., Kitagawa, K., Takebe, H., 1997. Mutation induction by high-density, 50$\mathrm{Hz}$ magnetic fields in human MeWo cells exposed in the DNA synthesis phase. International Journal of Radiation Biology. 71, 75-79.

Miyakoshi, J., Koji, Y., Wakasa, T., Takebe, H., 1999. Long-term exposure to a magnetic field $(5 \mathrm{mT}$ at $60 \mathrm{~Hz}$ ) increases X-ray-induced mutations. Journal of Radiation Research. 40, 13-21.

Miyakoshi, J., Yamagishi, N., Ohtsu, S., Mohri, K., Takebe, H., 1996. Increase in hypoxanthine-guanine phosphoribosyl transferase gene mutations by exposure to high-density 50-Hz magnetic fields. Mutation Research. 349, 109-114.

Miyakoshi, J., Yoshida, M., Shibuya, K., Hiraoka, M., 2000. Exposure to strong magnetic fields at power frequency potentiates $X$-ray-induced DNA strand breaks. Journal of Radiation Research. 41, 293-302.

Moore, R.Y., Heller, A., Wurtman, R.J., Axelrod, J., 1967. Visual pathway mediating pineal response to environmental light. Science. 155, 220-223.

Moretti, M., Villarini, M., Simonucci, S., Fatigoni, C., Scassellati-Sforzolini, G., Monarca, S., Pasquini, R., Angelucci, M., Strappini, M., 2005. Effects of co-exposure to extremely low frequency (ELF) magnetic fields and benzene or benzene metabolites determined in vitro by the alkaline comet assay. Toxicology Letters. 157, 119-128.

Morris, J.E., Sasser, L.B., Miller, D.L., Dagle, G.E., Rafferty, C.N., Ebi, K.L., Anderson, L.E., 1999. Clinical progression of transplanted large granular lymphocytic leukemia in Fischer 344 rats exposed to $60 \mathrm{~Hz}$ magnetic fields. Bioelectromagnetics. 20, 48-56.

Mughal, S.K., Myazin, A.E., Zhavoronkov, L.P., Rubanovich, A.V., Dubrova, Y.E., 2012. The dose and dose-rate effects of paternal irradiation on transgenerational instability in mice: a radiotherapy connection. PloS One. 7, 41300.

Muller-Sieburg, C.E. \& Riblet, R., 1996. Genetic control of the frequency of hematopoietic stem cells in mice: mapping of a candidate locus to chromosome 1 . The Journal of Experimental Medicine. 183, 1141-1150. 
Myers, A., Clayden, A.D., Cartwright, R.A., Cartwright, S.C., 1990. Childhood cancer and overhead powerlines: a case-control study. British Journal of Cancer. 62, 1008-1014.

Nakamura, Y., Tamura, H., Kashida, S., Takayama, H., Yamagata, Y., Karube, A., Sugino, N., Kato, H., 2001. Changes of serum melatonin level and its relationship to fetoplacental unit during pregnancy. Journal of Pineal Research. 30, 29-33.

Nault, R., Fader, K.A., Zacharewski, T., 2015. RNA-Seq versus oligonucleotide array assessment of dose-dependent TCDD-elicited hepatic gene expression in mice. BMC Genomics. 16, 373-015-1527-z.

Niwa, O., Fan, Y.J., Numoto, M., Kamiya, K., Kominami, R., 1996. Induction of a germline mutation at a hypervariable mouse minisatellite locus by $252 \mathrm{Cf}$ radiation. Journal of Radiation Research. 37, 217-224.

Noonan, C.W., Reif, J.S., Yost, M., Touchstone, J., 2002. Occupational exposure to magnetic fields in case-referent studies of neurodegenerative diseases. Scandinavian Journal of Work, Environment \& Health. 28, 42-48.

Nordenson, I., Mild, K.H., Andersson, G., Sandstrom, M., 1994. Chromosomal aberrations in human amniotic cells after intermittent exposure to fifty hertz magnetic fields. Bioelectromagnetics. 15, 293-301.

Ogheri, S., Rampazzo, C., Celotti, L., 1995. Mutagenic effects at hprt locus and in minisatellite sequences induced in V79 cells by treatments with UV and methyl-nitronitroso guanidine. Mutation Research. 348, 193-199.

Okamura, H., Yamaguchi, S., Yagita, K., 2002. Molecular machinery of the circadian clock in mammals. Cell and Tissue Research. 309, 47-56.

Olsen, J.H., Nielsen, A., Schulgen, G., 1993. Residence near high voltage facilities and risk of cancer in children. BMJ. 307, 891-895.

Orkin, S.H. \& Zon, L.I., 2008. Hematopoiesis: an evolving paradigm for stem cell biology. Cell. 132, 631-644.

Paile, W., Jokela, K., Koivistoinen, A., Salomaa, S., 1995. Effects of $50 \mathrm{~Hz}$ sinusoidal magnetic fields and spark discharges on human lymphocytes in vitro.

Bioelectrochemistry and Bioenergetics. 36, 15-22.

Panagopoulos, D.J., Karabarbounis, A., Lioliousis, C., 2013. ELF alternating magnetic field decreases reproduction by DNA damage induction. Cell Biochemistry and Biophysics. 67, 703-716.

Pandi-Perumal, S.,R., Srinivasan, ,V., Maestroni, G.J.,M., Cardinali, D.,P., Poeggeler, ,B., Hardeland, ,R., 2006. Melatonin. FEBS Journal. 273, 2813-2838. 
Patruno, A., Tabrez, S., Pesce, M., Shakil, S., Kamal, M.A., Reale, M., 2015. Effects of extremely low frequency electromagnetic field (ELF-EMF) on catalase, cytochrome P450 and nitric oxide synthase in erythro-leukemic cells. Life Sciences. 121, 117-123.

Pedersen, C., Raaschou-Nielsen, O., Rod, N.H., Frei, P., Poulsen, A.H., Johansen, C., Schuz, J., 2014. Distance from residence to power line and risk of childhood leukemia: a population-based case-control study in Denmark. Cancer Causes \& Control : CCC. 25, 171-177.

Phillips, J.L., 1993. Effects of electromagnetic field exposure on gene transcription. Journal of Cellular Biochemistry. 51, 381-386.

Phillips, J.L., Singh, N.P., Lai, H., 2009. Electromagnetic fields and DNA damage. Pathophysiology : The Official Journal of the International Society for Pathophysiology / ISP. 16, 79-88.

Pitkevitch, V.A., Ivanov, V.K., Tsyb, A.F., Maksyoutov, M.A., Matiash, V.A., Shchukina, N.V., 1997. Exposure levels for persons involved in recovery operations after the Chernobyl accident. Statistical analysis based on the data of the Russian National Medical and Dosimetric Registry (RNMDR). Radiation and Environmental Biophysics. 36, 149-160.

Polyzos, A., Parfett, C., Healy, C., Douglas, G., Yauk, C., 2006a. A single-molecule PCR approach to the measurement of induced expanded simple tandem repeat instability in vitro. Mutation Research. 594, 93-100.

Polyzos, A., Parfett, C., Healy, C., Douglas, G.R., Yauk, C.L., 2006b. Instability of expanded simple tandem repeats is induced in cell culture by a variety of agents: $\mathrm{N}$ Nitroso-N-ethylurea, benzo(a)pyrene, etoposide and okadaic acid. Mutation Research. 598, 73-84.

Portier C, Wolfe M, editors. NIEHS. 1998. Assessment of Health Effects from Exposure to Power-Line Frequency Electric and Magnetic Fields. NIH publication no. 98-3981. [Accessed 13 August 2015]. Available:

http://www.niehs.nih.gov/health/assets/docs_a_e/assessment_of_health_effects_fro m_exposure_to_powerline_frequency_electric_and_magnetic_fields.pdf.

Powell, S. \& McMillan, T.J., 1990. DNA damage and repair following treatment with ionizing radiation. Radiotherapy and Oncology : Journal of the European Society for Therapeutic Radiology and Oncology. 19, 95-108.

Preece, A.W., Grainger, P., Golding, J., Kaune, W., 1996. Domestic magnetic field exposures in Avon. Physics in Medicine and Biology. 41, 71-81.

Preece, A.W., Kaune, W., Grainger, P., Preece, S., Golding, J., 1997. Magnetic fields from domestic appliances in the UK. Physics in Medicine and Biology. 42, 67-76. 
Preston, D.L., Kusumi, S., Tomonaga, M., Izumi, S., Ron, E., Kuramoto, A., Kamada, N., Dohy, H., Matsuo, T., Matsui T [corrected to Matsuo,T.], 1994. Cancer incidence in atomic bomb survivors. Part III. Leukemia, lymphoma and multiple myeloma, 19501987. Radiation Research. 137, S68-97.

Preston-Martin, S., Gurney, J.G., Pogoda, J.M., Holly, E.A., Mueller, B.A., 1996. Brain tumor risk in children in relation to use of electric blankets and water bed heaters. Results from the United States West Coast Childhood Brain Tumor Study. American Journal of Epidemiology. 143, 1116-1122.

Pui, C.H., Robison, L.L., Look, A.T., 2008. Acute lymphoblastic leukaemia. Lancet. 371, 1030-1043.

Qiu, C., Fratiglioni, L., Karp, A., Winblad, B., Bellander, T., 2004. Occupational Exposure to Electromagnetic Fields and Risk of Alzheimer's Disease. Epidemiology. 15, 687-694.

Reiter, R.J., Tan, D.X., Mayo, J.C., Sainz, R.M., Leon, J., Czarnocki, Z., 2003. Melatonin as an antioxidant: biochemical mechanisms and pathophysiological implications in humans. Acta Biochimica Polonica. 50, 1129-1146.

Repacholi, M., 2012. Concern that "EMF" magnetic fields from power lines cause cancer. The Science of the Total Environment. 426, 454-458.

Ritz, C., Ruminski, W., Hougaard, K.S., Wallin, H., Vogel, U., Yauk, C.L., 2011. Germline mutation rates in mice following in utero exposure to diesel exhaust particles by maternal inhalation. Mutation Research. 712, 55-58.

Rodgers, C.T. \& Hore, P.J., 2009. Chemical magnetoreception in birds: the radical pair mechanism. Proceedings of the National Academy of Sciences of the United States of America. 106, 353-360.

Rogakou, E.P., Pilch, D.R., Orr, A.H., Ivanova, V.S., Bonner, W.M., 1998. DNA doublestranded breaks induce histone $\mathrm{H} 2 \mathrm{AX}$ phosphorylation on serine 139. The Journal of Biological Chemistry. 273, 5858-5868.

Roosli, M., Lortscher, M., Egger, M., Pfluger, D., Schreier, N., Lortscher, E., Locher, P., Spoerri, A., Minder, C., 2007. Mortality from neurodegenerative disease and exposure to extremely low-frequency magnetic fields: 31 years of observations on Swiss railway employees. Neuroepidemiology. 28, 197-206.

Rothman K. J., Greenland S., Lash T. L. Validity in epidemiologic studies. In: Rothman K. J., editor; Greenland S., editor; Lash T. L., editor. Modern Epidemiology 3rd edition. Philadelphia, PA: Lippincott Williams \& Wilkins; 2008c. pp. 128-147.

Ruiz-Gomez, M.J. \& Martinez-Morillo, M., 2009. Electromagnetic fields and the induction of DNA strand breaks. Electromagnetic Biology and Medicine. 28, 201-214. 
Russell, L.B., 2004. Effects of male germ-cell stage on the frequency, nature, and spectrum of induced specific-locus mutations in the mouse. Genetica. 122, 25-36.

Russell, L.B. \& Matter, B.E., 1980. Whole-mammal mutagenicity tests: evaluation of five methods. Mutation Research. 75, 279-302.

Russell, L.B. \& Russell, W.L., 1996. Spontaneous mutations recovered as mosaics in the mouse specific-locus test. Proceedings of the National Academy of Sciences of the United States of America. 93, 13072-13077.

Russell, W.L., 1989. Reminiscences of a mouse specific-locus test addict. Environmental and Molecular Mutagenesis. 14 Suppl 16, 16-22.

Russell, W.L., 1951. X-ray-induced mutations in mice. Cold Spring Harbor Symposia on Quantitative Biology. 16, 327-336.

Sadamoto, S., Suzuki, S., Kamiya, K., Kominami, R., Dohi, K., Niwa, O., 1994. Radiation induction of germline mutation at a hypervariable mouse minisatellite locus. International Journal of Radiation Biology. 65, 549-557.

Saha, S., Woodbine, L., Haines, J., Coster, M., Ricket, N., Barazzuol, L., Ainsbury, E., Sienkiewicz, Z., Jeggo, P., 2014. Increased apoptosis and DNA double-strand breaks in the embryonic mouse brain in response to very low-dose X-rays but not $50 \mathrm{~Hz}$ magnetic fields. Journal of the Royal Society, Interface / the Royal Society. 11, 20140783.

Sahl, J.D., Kelsh, M.A., Smith, R.W., Aseltine, D.A., 1994. Exposure to $60 \mathrm{~Hz}$ magnetic fields in the electric utility work environment. Bioelectromagnetics. 15, 21-32.

Sahl, J.D., Kelsh, M.A., Greenland, S., 1993. Cohort and Nested Case-Control Studies of Hematopoietic Cancers and Brain Cancer among Electric Utility Workers. Epidemiology. 4, 104-114.

Sahl, J., Mezei, G., Kavet, R., McMillan, A., Silvers, A., Sastre, A., Kheifets, L., 2002. Occupational magnetic field exposure and cardiovascular mortality in a cohort of electric utility workers. American Journal of Epidemiology. 156, 913-918.

Saito, T., Nitta, H., Kubo, O., Yamamoto, S., Yamaguchi, N., Akiba, S., Honda, Y., Hagihara, J., Isaka, K., Ojima, T., Nakamura, Y., Mizoue, T., Ito, S., Eboshida, A., Yamazaki, S., Sokejima, S., Kurokawa, Y., Kabuto, M., 2010. Power-Frequency Magnetic Fields and Childhood Brain Tumors: A Case-Control Study in Japan. Journal of Epidemiology. 20, 54-61.

Santini, M.T., Rainaldi, G., Indovina, P.L., 2009. Cellular effects of extremely low frequency (ELF) electromagnetic fields. International Journal of Radiation Biology. 85, 294-313. 
Sasser, L.B., Morris, J.E., Miller, D.L., Rafferty, C.N., Ebi, K.L., Anderson, L.E., 1996. Exposure to $60 \mathrm{~Hz}$ magnetic fields does not alter clinical progression of LGL leukemia in Fischer rats. Carcinogenesis. 17, 2681-2687.

Sastre, A., Cook, M.R., Graham, C., 1998. Nocturnal exposure to intermittent $60 \mathrm{~Hz}$ magnetic fields alters human cardiac rhythm. Bioelectromagnetics. 19, 98-106.

Satoh, C., Takahashi, N., Asakawa, J., Kodaira, M., Kuick, R., Hanash, S.M., Neel, J.V., 1996. Genetic analysis of children of atomic bomb survivors. Environmental Health Perspectives. 104 Suppl 3, 511-519.

Savitz, D.A., John, E.M., Kleckner, R.C., 1990. Magnetic field exposure from electric appliances and childhood cancer. American Journal of Epidemiology. 131, 763.

Savitz, D.A., Liao, D., Sastre, A., Kleckner, R.C., Kavet, R., 1999. Magnetic field exposure and cardiovascular disease mortality among electric utility workers. American Journal of Epidemiology. 149, 135.

Savitz, D.A., 1995. Exposure assessment strategies in epidemiological studies of health effects of electric and magnetic fields. The Science of the Total Environment. 168, 143153.

Savitz, D.A., Checkoway, H., Loomis, D.P., 1998. Magnetic field exposure and neurodegenerative disease mortality among electric utility workers. Epidemiology (Cambridge, Mass.). 9, 398-404.

Savitz, D.A., John, E.M., Kleckner, R.C., 1990. Magnetic field exposure from electric appliances and childhood cancer. American Journal of Epidemiology. 131, 763-773.

Savitz, D.A. \& Kaune, W.T., 1993. Childhood cancer in relation to a modified residential wire code. Environmental Health Perspectives. 101, 76-80.

Savitz, D.A. \& Loomis, D.P., 1995. Magnetic field exposure in relation to leukemia and brain cancer mortality among electric utility workers. American Journal of Epidemiology. 141, 123.

Savitz, D.A., Wachtel, H., Barnes, F.A., John, E.M., Tvrdik, J.G., 1988. Case-control study of childhood cancer and exposure to $60-\mathrm{Hz}$ magnetic fields. American Journal of Epidemiology. 128, 21-38.

Scarfi, M.R., Sannino, A., Perrotta, A., Sarti, M., Mesirca, P., Bersani, F., 2005. Evaluation of genotoxic effects in human fibroblasts after intermittent exposure to 50 $\mathrm{Hz}$ electromagnetic fields: a confirmatory study. Radiation Research. 164, 270-276.

Schoenfeld, E.R., O'Leary, E.S., Henderson, K., Grimson, R., Kabat, G.C., Ahnn, S., Kaune, W.T., Gammon, M.D., Leske, M.C., EBCLIS Group, 2003. Electromagnetic fields and breast cancer on Long Island: a case-control study. American Journal of Epidemiology. 158, 47-58. 
Schroeder, A., Mueller, O., Stocker, S., Salowsky, R., Leiber, M., Gassmann, M., Lightfoot, S., Menzel, W., Granzow, M., Ragg, T., 2006. The RIN: an RNA integrity number for assigning integrity values to RNA measurements. BMC Molecular Biology. $7,3$.

Schuz, J., Svendsen, A.L., Linet, M.S., McBride, M.L., Roman, E., Feychting, M., Kheifets, L., Lightfoot, T., Mezei, G., Simpson, J., Ahlbom, A., 2007. Nighttime exposure to electromagnetic fields and childhood leukemia: an extended pooled analysis. American Journal of Epidemiology. 166, 263-269.

Searle, A.G., 1974. Mutation Induction in Mice. Advances in Radiation Biology. 4, 131207.

Searle, A.G. \& Beechey, C.V., 1981. The effects of radiation dose-rate and quality on the induction of dominant lethals in mouse spermatids. Mutation Research. 81, 403410.

Selby, P.B., 1998. Discovery of numerous clusters of spontaneous mutations in the specific-locus test in mice necessitates major increases in estimates of doubling doses. Genetica. 102-103, 463-487.

Shanks, M., Riou, L., Fouchet, P., Dubrova, Y.E., 2008. Stage-specificity of spontaneous mutation at a tandem repeat DNA locus in the mouse germline. Mutation Research. $641,58-60$.

Shelby, M.D., 1996. Selecting chemicals and assays for assessing mammalian germ cell mutagenicity. Mutation Research. 352, 159-167.

Shen, Y.H., Shao, B.J., Chiang, H., Fu, Y.D., Yu, M., 1997. The effects of $50 \mathrm{~Hz}$ magnetic field exposure on dimethylbenz(alpha)anthracene induced thymic lymphoma/leukemia in mice. Bioelectromagnetics. 18, 360-364.

Simko, M., Kriehuber, R., Weiss, D.G., Luben, R.A., 1998. Effects of $50 \mathrm{~Hz}$ EMF exposure on micronucleus formation and apoptosis in transformed and nontransformed human cell lines. Bioelectromagnetics. 19, 85-91.

Simko, M. \& Mattsson, M.O., 2004. Extremely low frequency electromagnetic fields as effectors of cellular responses in vitro: possible immune cell activation. Journal of Cellular Biochemistry. 93, 83-92.

Singer, T.M. \& Yauk, C.L., 2010. Germ cell mutagens: risk assessment challenges in the 21st century. Environmental and Molecular Mutagenesis. 51, 919-928.

Singh, M. \& Jadhav, H.R., 2014. Melatonin: functions and ligands. Drug Discovery Today. 19, 1410-1418.

Singh, N. \& Lai, H., 1998. $60 \mathrm{~Hz}$ magnetic field exposure induces DNA crosslinks in rat brain cells. Mutation Research. 400, 313-320. 
Slominski, R.M., Reiter, R.J., Schlabritz-Loutsevitch, N., Ostrom, R.S., Slominski, A.T., 2012. Melatonin membrane receptors in peripheral tissues: distribution and functions. Molecular and Cellular Endocrinology. 351, 152-166.

Slominski, A., Tobin, D.J., Zmijewski, M.A., Wortsman, J., Paus, R., 2008. Melatonin in the skin: synthesis, metabolism and functions. Trends in Endocrinology and Metabolism: TEM. 19, 17-24.

Sobel, E., Davanipour, Z., Sulkava, R., Erkinjuntti, T., Wikstrom, J., Henderson, V.W., Buckwalter, G., Bowman, J.D., Lee, P.J., 1995. Occupations with exposure to electromagnetic fields: a possible risk factor for Alzheimer's disease. American Journal of Epidemiology. 142, 515.

Somers, C.M., 2006. Expanded simple tandem repeat (ESTR) mutation induction in the male germline: lessons learned from lab mice. Mutation Research. 598, 35-49.

Somers, C.M., McCarry, B.E., Malek, F., Quinn, J.S., 2004. Reduction of particulate air pollution lowers the risk of heritable mutations in mice. Science. 304, 1008-1010.

Somers, C.M., Yauk, C.L., White, P.A., Parfett, C.L., Quinn, J.S., 2002. Air pollution induces heritable DNA mutations. Proceedings of the National Academy of Sciences of the United States of America. 99, 15904-15907.

Sorahan, T. \& Mohammed, N., 2014. Neurodegenerative disease and magnetic field exposure in UK electricity supply workers. Occupational Medicine. 64, 454.

Southern, E.M., 1975. Detection of specific sequences among DNA fragments separated by gel electrophoresis. Journal of Molecular Biology. 98, 503-517.

Southern, E.M., 1979. Measurement of DNA length by gel electrophoresis. Analytical Biochemistry. 100, 319-323.

Stevens, R.G., 1987. Electric power use and breast cancer: a hypothesis. American Journal of Epidemiology. 125, 556-561.

Stevens, R.G. \& Davis, S., 1996. The melatonin hypothesis: electric power and breast cancer. Environmental Health Perspectives. 104 Suppl 1, 135-140.

Stronati, L., Testa, A., Villani, P., Marino, C., Lovisolo, G.A., Conti, D., Russo, F., Fresegna, A.M., Cordelli, E., 2004. Absence of genotoxicity in human blood cells exposed to $50 \mathrm{~Hz}$ magnetic fields as assessed by comet assay, chromosome aberration, micronucleus, and sister chromatid exchange analyses. Bioelectromagnetics. 25, 41-48.

Swanson, J. \& Kaune, W.T., 1999. Comparison of residential power-frequency magnetic fields away from appliances in different countries. Bioelectromagnetics. 20, 244-254. 
Tateno, H., lijima, S., Nakanishi, Y., Kamiguchi, Y., Asaka, A., 1998. No induction of chromosome aberrations in human spermatozoa exposed to extremely low frequency electromagnetic fields. Mutation Research. 414, 31-35.

Teepen, J.C. \& van Dijck, J.A., 2012. Impact of high electromagnetic field levels on childhood leukemia incidence. International Journal of Cancer.Journal International Du Cancer. 131, 769-778.

Tenforde, T.S., 1992. Biological interactions and potential health effects of extremelylow-frequency magnetic fields from power lines and other common sources. Annual Review of Public Health. 13, 173-196.

Testa, A., Cordelli, E., Stronati, L., Marino, C., Lovisolo, G.A., Fresegna, A.M., Conti, D., Villani, P., 2004. Evaluation of genotoxic effect of low level $50 \mathrm{~Hz}$ magnetic fields on human blood cells using different cytogenetic assays. Bioelectromagnetics. 25, 613619.

Timmel, C.R., Till, U., Brocklehurst, B., Mclauchlan, K.A., Hore, P.J., 1998. Effects of weak magnetic fields on free radical recombination reactions. Molecular Physics: An International Journal at the Interface between Chemistry and Physics. 95, 71-89.

Tomenius, L., 1986. 50- $\mathrm{Hz}$ electromagnetic environment and the incidence of childhood tumors in Stockholm County. Bioelectromagnetics. 7, 191-207.

Touitou, Y., Bogdan, A., Lambrozo, J., Selmaoui, B., 2006. Is melatonin the hormonal missing link between magnetic field effects and human diseases? Cancer Causes \& Control : CCC. 17, 547-552.

Touitou, Y., Lambrozo, J., Camus, F., Charbuy, H., 2003. Magnetic fields and the melatonin hypothesis: A study of workers chronically exposed to $50-\mathrm{Hz}$ magentic fields. American Journal of Physiology. 53, R1529.

Tynes, T. \& Haldorsen, T., 1997. Electromagnetic fields and cancer in children residing near Norwegian high-voltage power lines. American Journal of Epidemiology. 145, 219226.

Udroiu, I., Giuliani, L., leradi, L.A., 2010. Genotoxic properties of extremely low frequency electromagnetic fields. In: Giuliani, L., Soffritti, M. (eds). Non-thermal Effects and Mechanisms of Interaction between Electromagnetic Fields and Living Matter. National Institute for the Study and Control of Cancer and Environmental Diseases, Bernardino Ramazzini, Bologna: Mattioli 1885, 123-134.

(UKCC) UK Childhood Cancer Study Investigators, 1999. Exposure to power-frequency magnetic fields and the risk of childhood cancer. UK Childhood Cancer Study Investigators. Lancet. 354, 1925-1931.

(UKCC) UK Childhood Cancer Study Investigators, 2000. Childhood cancer and residential proximity to power lines. British Journal of Cancer. 83, 1573-1580. 
(UNSCEAR) United Nations Scientific Committee on the Effects of Atomic Radiation. 2001. Hereditary Effects of Radiation. [Online]. Report to the General Assembly, with Scientific Annex. New York: United Nations. [01/09/2014]. Available from: http://www.unscear.org/docs/reports/2001/2001Annex_pages\%208-160.pdf

Vanderstraeten, J., Verschaeve, L., Burda, H., Bouland, C., de Brouwer, C., 2012. Health effects of extremely low-frequency magnetic fields: reconsidering the melatonin hypothesis in the light of current data on magnetoreception. Journal of Applied Toxicology : JAT. 32, 952-958.

Vena, J.E., Freudenheim, J.L., Marshall, J.R., Laughlin, R., Swanson, M., Graham, S., 1994. Risk of premenopausal breast cancer and use of electric blankets. American Journal of Epidemiology. 140, 974.

Vena, J.E., Graham, S., Hellmann, R., Swanson, M., Brasure, J., 1991. Use of electric blankets and risk of postmenopausal breast cancer. American Journal of Epidemiology. 134, 180.

Vergara, X., Kheifets, L., Greenland, S., Oksuzyan, S., Cho, Y., Mezei, G., 2013. Occupational Exposure to Extremely Low-Frequency Magnetic Fields and Neurodegenerative Disease: A Meta-Analysis. Journal of Occupational and Environmental Medicine. 55, 135-146.

Vergara, X., Mezei, G., Kheifets, L., 2015. Case-control study of occupational exposure to electric shocks and magnetic fields and mortality from amyotrophic lateral sclerosis in the US, 1991-1999. Journal of Exposure Science \& Environmental Epidemiology. 25, 65-71.

Vergnaud, G., Mariat, D., Apiou, F., Aurias, A., Lathrop, M., Lauthier, V., 1991. The use of synthetic tandem repeats to isolate new VNTR loci: cloning of a human hypermutable sequence. Genomics. 11, 135-144.

Verkasalo, P.K., Pukkala, E., Hongisto, M.Y., Valjus, J.E., Jarvinen, P.J., Heikkila, K.V., Koskenvuo, M., 1993. Risk of cancer in Finnish children living close to power lines. BMJ. 307, 895-899.

Verkasalo, P.K., Pukkala, E., Kaprio, J., Heikkila, K.V., Koskenvuo, M., 1996. Magnetic fields of high voltage power lines and risk of cancer in Finnish adults: nationwide cohort study. BMJ. 313, 1047-1051.

Vijayalaxmi \& Obe, G., 2005. Controversial cytogenetic observations in mammalian somatic cells exposed to extremely low frequency electromagnetic radiation: a review and future research recommendations. Bioelectromagnetics. 26, 412-430.

Vijayalaxmi \& Prihoda, T.J., 2009. Genetic damage in mammalian somatic cells exposed to extremely low frequency electro-magnetic fields: a meta-analysis of data from 87 publications (1990-2007). International Journal of Radiation Biology. 85, 196-213. 
Vilarino-Guell, C., Smith, A.G., Dubrova, Y.E., 2003. Germline mutation induction at mouse repeat DNA loci by chemical mutagens. Mutation Research. 526, 63-73.

Villarini, M., Moretti, M., Scassellati-Sforzolini, G., Boccioli, B., Pasquini, R., 2006. Effects of co-exposure to extremely low frequency $(50 \mathrm{~Hz})$ magnetic fields and xenobiotics determined in vitro by the alkaline comet assay. The Science of the Total Environment. 361, 208-219.

Voutounou, M., Glen, C.D., Dubrova, Y.E., 2012. The effects of methyl-donor deficiency on mutation induction and transgenerational instability in mice. Mutation Research. 734, 1-4.

Walleczek, J., Shiu, E.C., Hahn, G.M., 1999. Increase in radiation-induced HPRT gene mutation frequency after nonthermal exposure to nonionizing $60 \mathrm{~Hz}$ electromagnetic fields. Radiation Research. 151, 489-497.

Wang, Z., Gerstein, M., Snyder, M., 2009. RNA-Seq: a revolutionary tool for transcriptomics. Nature Reviews.Genetics. 10, 57-63.

Wartenberg, D., 1998. Residential magnetic fields and childhood leukemia: a metaanalysis. American Journal of Public Health. 88, 1787-1794.

Wartenberg, D., Ehrlich, R., Lilienfeld, D., 1994. Environmental tobacco smoke and childhood asthma: comparing exposure metrics using probability plots. Environmental Research. 64, 122-135.

Weitzmann, M.N., Woodford, K.J., Usdin, K., 1998. The mouse Ms6-hm hypervariable microsatellite forms a hairpin and two unusual tetraplexes. The Journal of Biological Chemistry. 273, 30742-30749.

Welker, H.A., Semm, P., Willig, R.P., Commentz, J.C., Wiltschko, W., Vollrath, L., 1983. Effects of an artificial magnetic field on serotonin $\mathrm{N}$-acetyltransferase activity and melatonin content of the rat pineal gland. Experimental Brain Research. 50, 426-432.

Wertheimer, N. \& Leeper, E., 1982. Adult cancer related to electrical wires near the home. International Journal of Epidemiology. 11, 345-355.

Wertheimer, N. \& Leeper, E., 1979. Electrical wiring configurations and childhood cancer. American Journal of Epidemiology. 109, 273-284.

(WHO) World Health Organization. 2007. Extremely Low Frequency Fields. [Online]. Environmental Health Criteria Monograph No.238. WHO Spain: WHO Press. [26/01/2015]. Available from: http://www.who.int/pehemf/publications/elf_ehc/en/index.html.

Wilbourn, J., Haroun, L., Heseltine, E., Kaldor, J., Partensky, C., Vainio, H., 1986. Response of experimental animals to human carcinogens: an analysis based upon the IARC Monographs programme. Carcinogenesis. 7, 1853-1863. 
Wilson, B.W., Lee, G.M., Yost, M.G., Davis, K.C., Heimbigner, T., Buschbom, R.L., 1996. Magnetic field characteristics of electric bed-heating devices. Bioelectromagnetics. 17, 174-179.

Wilson, J.W., Haines, J., Sienkiewicz, Z., Dubrova, Y.E., 2015. The effects of extremely low frequency magnetic fields on mutation induction in mice. Mutation Research. 773, 22-26.

Winker, R., Ivancsits, S., Pilger, A., Adlkofer, F., Rudiger, H.W., 2005. Chromosomal damage in human diploid fibroblasts by intermittent exposure to extremely lowfrequency electromagnetic fields. Mutation Research. 585, 43-49.

Witt, K.L. \& Bishop, J.B., 1996. Mutagenicity of anticancer drugs in mammalian germ cells. Mutation Research. 355, 209-234.

Wolf, F.I., Torsello, A., Tedesco, B., Fasanella, S., Boninsegna, A., D'Ascenzo, M., Grassi, C., Azzena, G.B., Cittadini, A., 2005. 50-Hz extremely low frequency electromagnetic fields enhance cell proliferation and DNA damage: possible involvement of a redox mechanism. Biochimica Et Biophysica Acta. 1743, 120-129.

Yaguchi, H., Yoshida, M., Ding, G.R., Shingu, K., Miyakoshi, J., 2000. Increased chromatid-type chromosomal aberrations in mouse $\mathrm{m} 5 \mathrm{~S}$ cells exposed to power-line frequency magnetic fields. International Journal of Radiation Biology. 76, 1677-1684.

Yaguchi, H., Yoshida, M., Ejima, Y., Miyakoshi, J., 1999. Effect of high-density extremely low frequency magnetic field on sister chromatid exchanges in mouse m5S cells.

Mutation Research. 440, 189-194.

Yasui, M., Kikuchi, T., Ogawa, M., Otaka, Y., Tsuchitani, M., Iwata, H., 1997. Carcinogenicity test of $50 \mathrm{~Hz}$ sinusoidal magnetic fields in rats. Bioelectromagnetics. 18, 531-540.

Yauk, C., Polyzos, A., Rowan-Carroll, A., Somers, C.M., Godschalk, R.W., Van Schooten, F.J., Berndt, M.L., Pogribny, I.P., Koturbash, I., Williams, A., Douglas, G.R., Kovalchuk, O., 2008. Germ-line mutations, DNA damage, and global hypermethylation in mice exposed to particulate air pollution in an urban/industrial location. Proceedings of the National Academy of Sciences of the United States of America. 105, 605-610.

Yauk, C.L., 2004. Advances in the application of germline tandem repeat instability for in situ monitoring. Mutation Research. 566, 169-182.

Yauk, C.L., Aardema, M.J., Benthem, J., Bishop, J.B., Dearfield, K.L., DeMarini, D.M., Dubrova, Y.E., Honma, M., Lupski, J.R., Marchetti, F., Meistrich, M.L., Pacchierotti, F., Stewart, J., Waters, M.D., Douglas, G.R., 2015. Approaches for identifying germ cell mutagens: Report of the 2013 IWGT workshop on germ cell assays. Mutation Research.Genetic Toxicology and Environmental Mutagenesis. 783, 36-54. 
Yauk, C.L., Berndt, M.L., Williams, A., Rowan-Carroll, A., Douglas, G.R., Stampfli, M.R., 2007. Mainstream tobacco smoke causes paternal germ-line DNA mutation. Cancer Research. 67, 5103-5106.

Yauk, C.L., Dubrova, Y.E., Grant, G.R., Jeffreys, A.J., 2002. A novel single molecule analysis of spontaneous and radiation-induced mutation at a mouse tandem repeat locus. Mutation Research. 500, 147-156.

Yauk, C.L., Fox, G.A., McCarry, B.E., Quinn, J.S., 2000. Induced minisatellite germline mutations in herring gulls (Larus argentatus) living near steel mills. Mutation Research. 452, 211-218.

Yauk, C.L. \& Quinn, J.S., 1996. Multilocus DNA fingerprinting reveals high rate of heritable genetic mutation in herring gulls nesting in an industrialized urban site. Proceedings of the National Academy of Sciences of the United States of America. 93, 12137-12141.

Yauk, C. \& Polyzos, A., 2005. Tandem repeat DNA: applications in mutation analysis. Environmental Mutagen Research. 27, 93-98.

Yokus, B., Cakir, D.U., Akdag, M.Z., Sert, C., Mete, N., 2005. Oxidative DNA damage in rats exposed to extremely low frequency electromagnetic fields. Free Radical Research. 39, 317-323.

Yoon, H.E., Lee, J.S., Myung, S.H., Lee, Y.S., 2014. Increased gamma-H2AX by exposure to a $60-\mathrm{Hz}$ magnetic fields combined with ionizing radiation, but not hydrogen peroxide, in non-tumorigenic human cell lines. International Journal of Radiation Biology. 90, 291-298.

Zaffanella, L.E. (1992). Survey of residential magnetic field sources. EPRI Report. United States. http://www.epri.com/abstracts/Pages/ProductAbstract.aspx?Productld=TR100194, Last visited 8/12/2015

Zhao, L., Liu, X., Wang, C., Yan, K., Lin, X., Li, S., Bao, H., Liu, X., 2014. Magnetic fields exposure and childhood leukemia risk: a meta-analysis based on 11,699 cases and 13,194 controls. Leukemia Research. 38, 269-274.

Zheng, N., Monckton, D.G., Wilson, G., Hagemeister, F., Chakraborty, R., Connor, T.H., Siciliano, M.J., Meistrich, M.L., 2000a. Frequency of minisatellite repeat number changes at the MS205 locus in human sperm before and after cancer chemotherapy. Environmental and Molecular Mutagenesis. 36, 134-145.

Zheng, T., Holford, T.R., Mayne, S.T., Owens, P.H., Zhang, B., Boyle, P., Carter, D., Ward, B., Zhang, Y., Zahm, S.H., 2000b. Exposure to electromagnetic fields from use of electric blankets and other in-home electrical appliances and breast cancer risk. American Journal of Epidemiology. 151, 1103-1111. 
Zhou, H., Chen, G., Chen, C., Yu, Y., Xu, Z., 2012. Association between extremely lowfrequency electromagnetic fields occupations and amyotrophic lateral sclerosis: a meta-analysis. PloS One. 7, 48354.

Zhou, Y., Liu, Y., Qiu, W., Zeng, J., Chen, X., Zhou, H., Li, A., Zhou, J., 2009. Exposure to residential indoor air induces heritable DNA mutations in mice. Journal of Toxicology and Environmental Health.Part A. 72, 1561-1566.

Zmyslony, M., Palus, J., Jajte, J., Dziubaltowska, E., Rajkowska, E., 2000. DNA damage in rat lymphocytes treated in vitro with iron cations and exposed to $7 \mathrm{mT}$ magnetic fields (static or $50 \mathrm{~Hz}$ ). Mutation Research. 453, 89-96.

Zmyslony, M., Rajkowska, E., Mamrot, P., Politanski, P., Jajte, J., 2004. The effect of weak $50 \mathrm{~Hz}$ magnetic fields on the number of free oxygen radicals in rat lymphocytes in vitro. Bioelectromagnetics. 25, 607-612. 